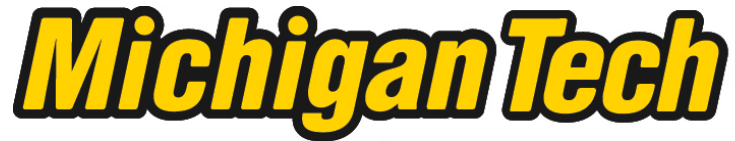 \\ Michigan Technological University Create the Future Digital Commons @ Michigan Tech
}

Dissertations, Master's Theses and Master's Reports - Open

Dissertations, Master's Theses and Master's

Reports

2009

Immobilizing bacteriorhodopsin on a single electron transistor

Karl A. Walczak

Michigan Technological University

Follow this and additional works at: https://digitalcommons.mtu.edu/etds

Part of the Mechanical Engineering Commons

Copyright 2009 Karl A. Walczak

\section{Recommended Citation}

Walczak, Karl A., "Immobilizing bacteriorhodopsin on a single electron transistor", Dissertation, Michigan Technological University, 2009.

https://doi.org/10.37099/mtu.dc.etds/421

Follow this and additional works at: https://digitalcommons.mtu.edu/etds

Part of the Mechanical Engineering Commons 


\title{
Immobilizing Bacteriorhodopsin on a Single Electron Transistor
}

\author{
By \\ Karl A. Walczak \\ A DISSERTATION \\ Submitted in partial fulfillment of the requirements \\ For the degree of \\ DOCTOR OF PHILOSOPHY \\ (Mechanical Engineering - Engineering Mechanics) \\ Michigan Technological University \\ 2009
}

Copyright (C) Karl A. Walczak 
MICHIGAN TECHNOLOGICAL UNIVERSITY

DEPARTMENT OF MECHANICAL ENGINEERING

ENGINEERING MECHANICS

WE HEREBY RECOMMEND THAT THIS DISSERTATION BY

Karl A. Walczak

\section{ENTITLED}

Immobilizing Bacteriorhodopsin on a Single Electron Transistor

BE APPROVED IN PARTIAL FULFILLMENT OF THE REQUIREMENTS

FOR THE DEGREE OF

Doctor of Philosophy in Mechanical Engineering-Engineering Mechanics

\begin{tabular}{lc}
\hline Dissertation Advisor: Dr. Craig R. Friedrich & Date \\
& \\
\hline Department Chair: Dr. William W. Predebon & Date
\end{tabular}

Committee on Final Examination

Dr. Craig R. Friedrich (Chair, Mech. Eng.-Eng. Mechs.)

Dr. Donald R. Lueking (Biological Sciences)

Dr. Paul L. Bergstrom (Electrical and Computer Engineering)

Dr. Michele H. Miller (Mech. Eng.-Eng. Mechs.)

Dr. John W. Sutherland (Mech. Eng.-Eng. Mechs.) 


\section{ACKNOWLEDGEMENTS}

Thank you to Dr. Craig Friedrich for giving me the opportunity to work with you, I have enjoyed it thoroughly. The countless hours you have spent helping me through my $\mathrm{Ph} . \mathrm{D}$ have not gone unnoticed or unappreciated. Thank you for providing many opportunities for growth in my professional career.

Thank you to Dr. Don Lueking for welcoming me into the biology department and for providing guidance and expertise both in the lab and out of the lab.

Drs. Paul Bergstrom, Michele Miller, and John Sutherland, thank you for being a member of my committee. Thank you for your time, insight, and guidance into my research.

Thank you to Michel Chase for welcoming me into the electrical engineering department and for the introduction into circuit analysis and fabrication.

Thank you to William Knudsen, Owen Mills, John Miller and Mark Kilpela for your professional and technical expertise, I have learned a lot from you.

Thank you to my fellow graduate students especially; Dr. Chris Anton, Dr. Mark Griep, Eric Winder, Dr. Santosh Karre, Daw Don Cheam, and Dr. Manoranjan Acharya.

Thank you to the Michigan Tech cross-country running and nordic ski teams for your support and stress relief.

Thank you to John and Greta Hutchins for your support and encouragement.

Thank you to my family, especially my parents Walter and Julie Walczak for your love, support, and encouragement.

Thank you to Margot Hutchins for your unconditional love, support, and encouragement.

The research reported in this document was performed in connection with contract DAAD17-03-C-0115 with the U.S. Army Research Laboratory. The views and conclusions contained in this document are those of the authors and should not be interpreted as presenting the official policies or position, either expressed or implied, of the U.S. Army Research Laboratory or the U.S. Government unless so designated by other authorized documents. Citation of manufacturer's or trade names does not constitute an official endorsement or approval of the use thereof. The U.S. Government is authorized to reproduce and distribute reprints for Government purposes notwithstanding any copyright notation hereon. 


\section{ABSTRACT}

As awareness of potential human and environmental impacts from toxins has increased, so has the development of innovative sensors. Bacteriorhodopsin (bR) is a light activated proton pump contained in the purple membrane (PM) of the bacteria Halobacterium salinarum. Bacteriorhodopsin is a robust protein which can function in both wet and dry states and can withstand extreme environmental conditions. A single electron transistor (SET) is a nano-scale device that exploits the quantum mechanical properties of electrons to switch on and off. SETs have tremendous potential in practical applications due to their size, ultra low power requirements, and electrometer-like sensitivity.

The main goal of this research was to create a bionanohybrid device by integrating $b R$ with a SET device. This was achieved by a multidisciplinary approach. The SET devices were created by a combination of sputtering, photolithography, and focused ion beam machining. The bionanomaterial bacteriorhodopsin was created through oxidative fermentation and a series of transmembrane purification processes. The $b R$ was then integrated with the SET by electrophoretic deposition, creating a bionanohybrid device. The bionanohybrid device was then characterized using a semiconductor parametric analyzer. Characterization demonstrated that the bR modulated the operational characteristics of the SET when bR was activated with light within its absorbance spectrum.

To effectively integrate bacteriorhodopsin with microelectromechanical systems (MEMS) and nanoelectromechanical systems (NEMS), it is critical to know the electrical properties of the material and to understand how it will affect the functionality of the device. Tests were performed on dried films of $b R$ to determine if there is a relationship between inductance, capacitance, and resistance (LCR) measurements and orientation, light-on/off, frequency, and time. The results indicated that the LCR measurements of the $\mathrm{bR}$ depended on the thickness and area of the film, but not on the orientation, as with other biological materials such as muscle. However, there was a transient LCR response for both oriented and unoriented bR which depended on light intensity.

From the impedance measurements an empirical model was suggested for the bionanohybrid device. The empirical model is based on the dominant electrical characteristics of the bR which were the parallel capacitance and resistance. The empirical model suggests that it is possible to integrate bR with a SET without influencing its functional characteristics. 


\section{TABLE OF CONTENTS}

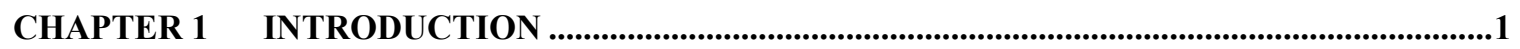

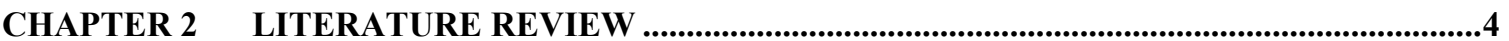

2.1 Overview of Halobacterium, Purple Membrane, and Bacteriorhodopsin....................................4

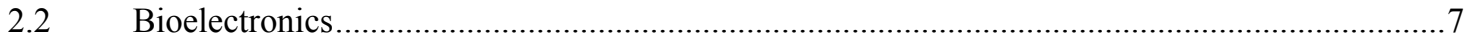

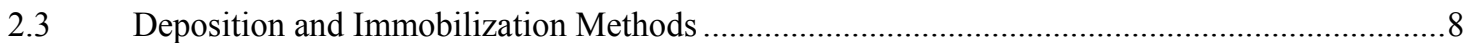

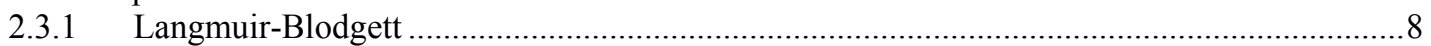

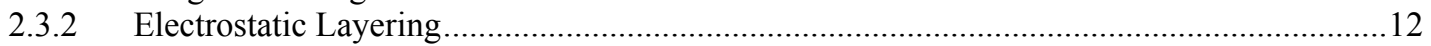

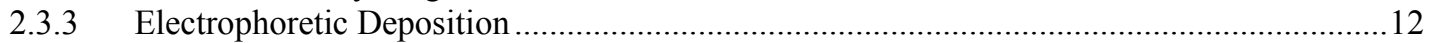

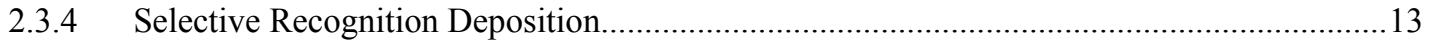

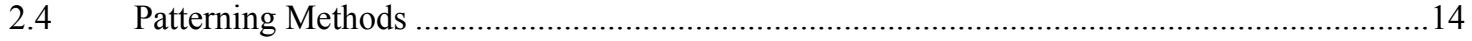

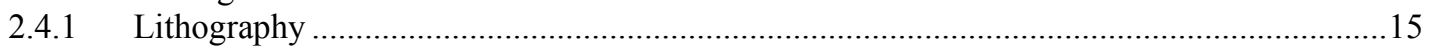

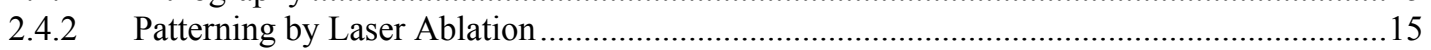

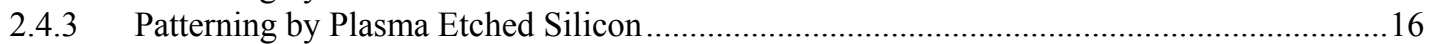

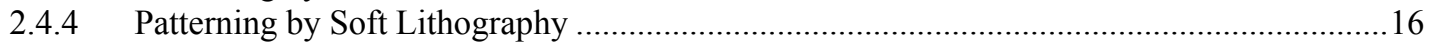

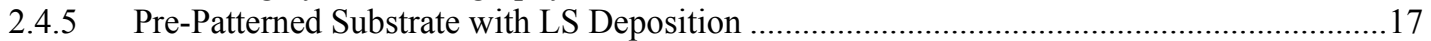

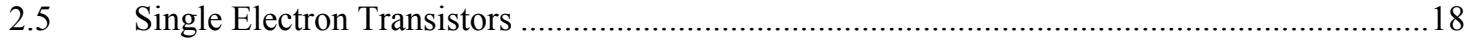

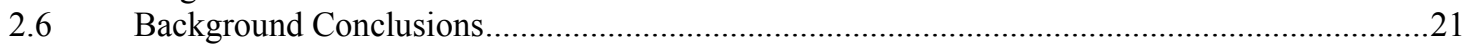

CHAPTER 3 PURIFICATION AND VERIFICATION OF PURPLE MEMBRANE.....................22

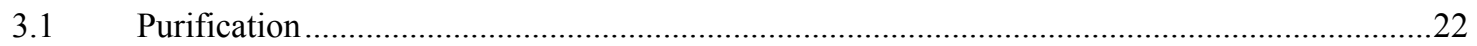

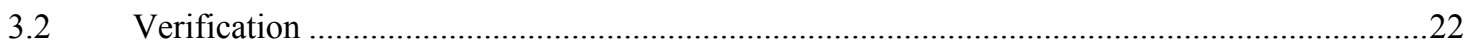

3.3 Purification and Verification of Purple Membrane Conclusions...............................................29

CHAPTER 4 PURPLE MEMBRANE MINIMIZATION AND MONOMERIZATION..................30

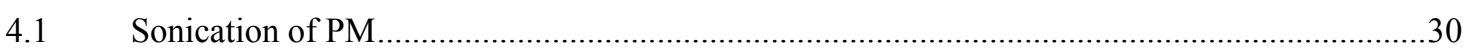

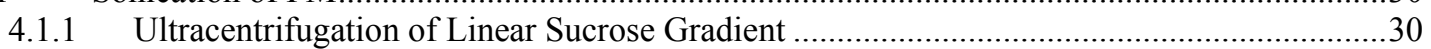

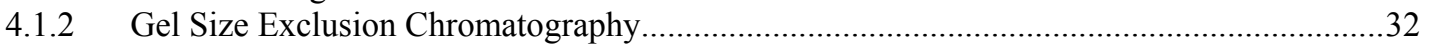

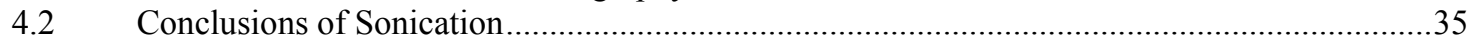

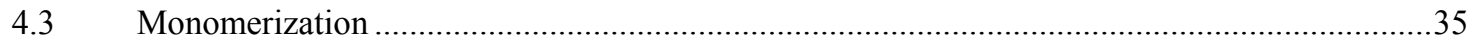

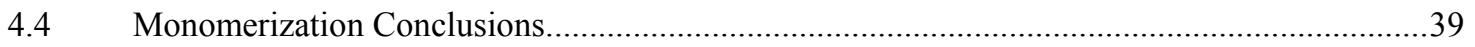

CHAPTER 5 BIOTINYLATION, DEPOSITION, AND ACTIVATION ..........................................42

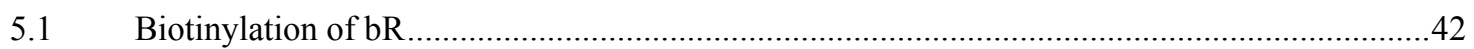

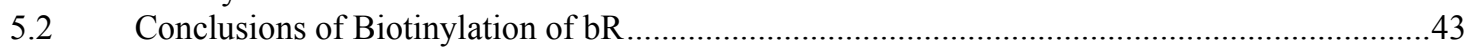

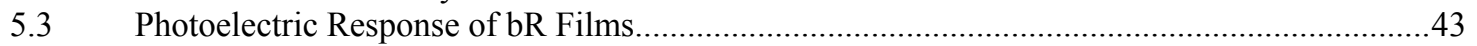

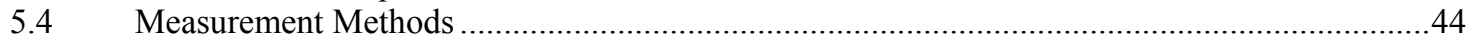

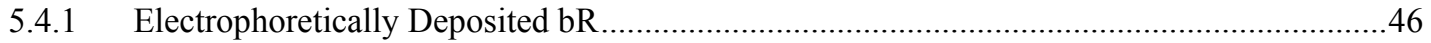

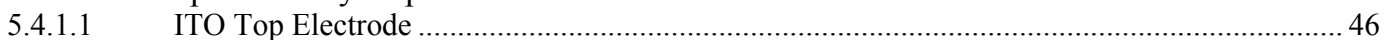

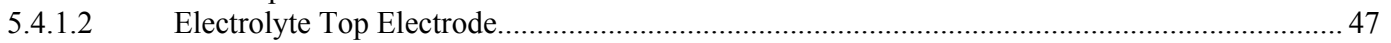

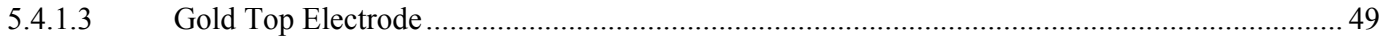

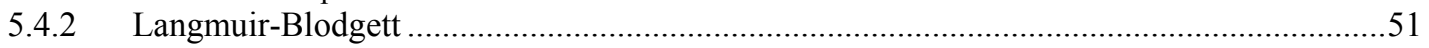

CHAPTER 6 ELECTRONIC PROPERTIES OF DRIED FILMS- BACTERIORHODOPSIN

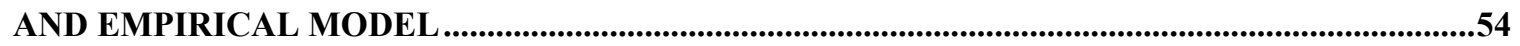

6.1 Impedance Measurements and Suggested Empirical Model ...................................................54

6.2 Operational Amplifier Circuit Measurements and Empirical Model ..........................................61

6.2.1 Light Activated RC Time Constant Based on Photovoltaic Response ...................................61

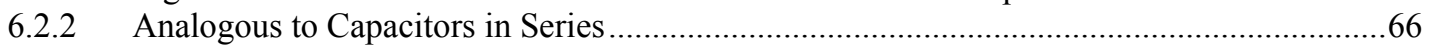

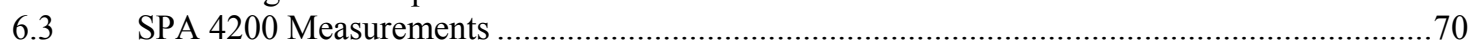


6.3.1 I-V Characteristic of a Multi-Monolayer bR Structure........................................................

\section{CHAPTER 7 FABRICATION OF SINGLE ELECTRON TRANSISTORS .....................................74}

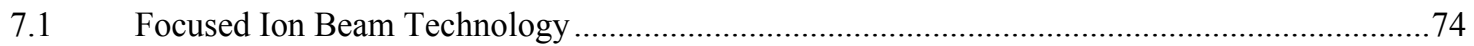

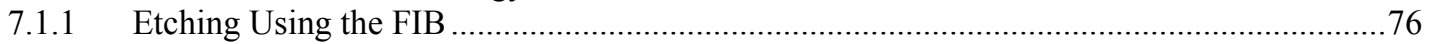

7.1.2 Deposition of Tungsten Quantum Islands Using FIB .........................................................

7.2 SET Fabrication with Interface Pad for Bacteriorhodopsin ......................................................

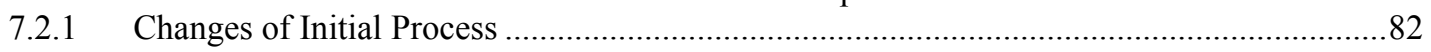

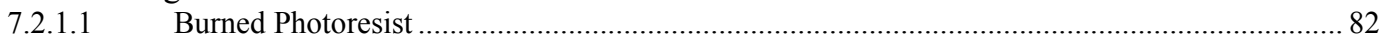

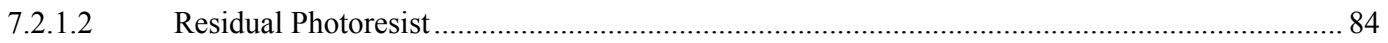

7.2.1.3 Chromium Thin Film ......................................................................................... 85

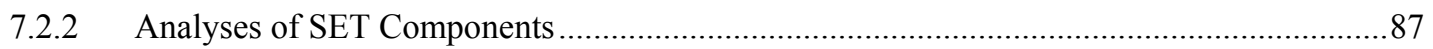

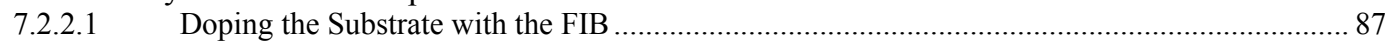

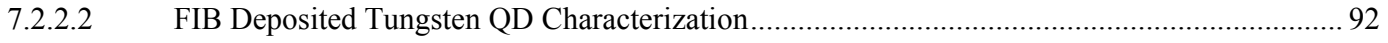

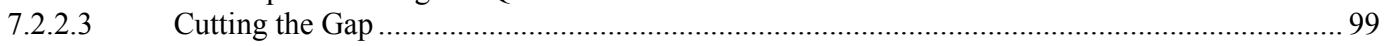

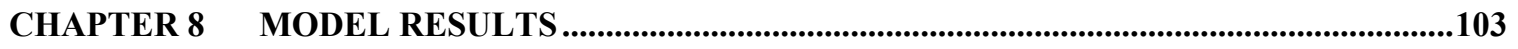

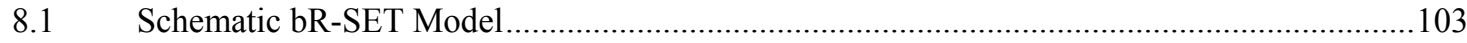

8.2 Activation of bR-SET Without Sputtered Gold Electrode ......................................................104

8.3 Activation of bR-SET with Sputter Gold Counter-Electrode ...................................................109

CHAPTER 9 CONCLUSIONS AND RECOMMENDATIONS ...................................................112

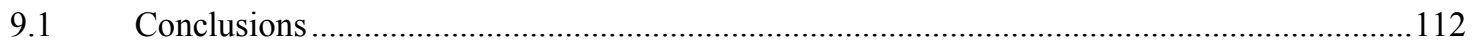

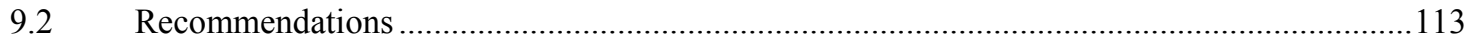




\section{LIST OF FIGURES}

Figure 1. Process flow for development of a molecular electronic device............................................

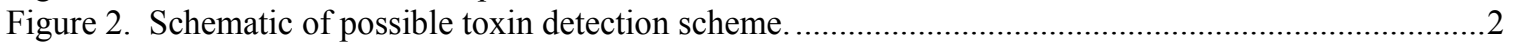

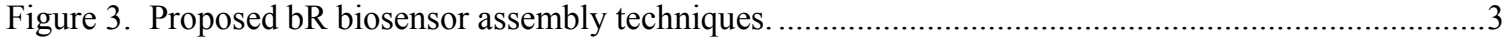

Figure 4. Depiction of proton pumping and ATP synthesis in Halobacterium salinarum. .........................5

Figure 5. Nobel contributions integrated into this project. .............................................................

Figure 6. Idealized deposition of amphiphilic monolayers with different deposition arrangements.............9

Figure 7. LB deposition of bR onto a solid substrate in the presence of an electric field. The polarity of the electrodes $\mathrm{A}$ and $\mathrm{B}$ control the bR orientation.................................................................... 10

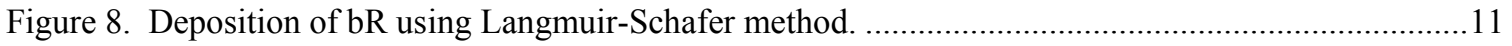

Figure 9. Sequence of modified horizontal deposition technique. ..................................................... 11

Figure 10. Scheme of PDAC/PM layering on negatively charged substrate.........................................12

Figure 11. Schematic representation of electrophoretic deposition and image of bR in phospholipid matrix.

13

Figure 12. Possible layering scenario for Biotin and Streptavidin interaction. ...................................... 14

Figure 13. Schematic representation of the pre-patterned pixellated array of bR photoreceptors from [57].

Figure 14. Schematic representation of a single electron transistor

Figure 15. Gate conductance oscillations at a source-drain bias of $25 \mathrm{mV}$. The upper curve is the reverse sweep of the lower curve and is offset by $1.4 \mathrm{nA}$. Both side gates are biased together. (b) The current-voltage characteristic and differential conductance at $4.2 \mathrm{~K}$. [64] ............................20

Figure 16. Final purification step, the $b R$ was fractioned in the linear sucrose gradient..........................23

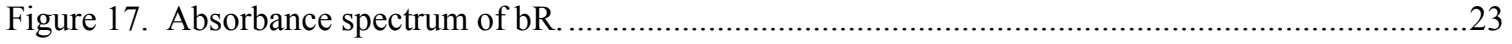

Figure 18. SDS gel, Lane a - Mark12 standard, Lane b - $2 \mu \mathrm{g}$ purified bR post-sucrose gradient, Lane $\mathrm{c}$ and $\mathrm{e}-\sim 2 \mu \mathrm{g}$ biotinylated $\mathrm{bR}$, Lane $\mathrm{d}$ and $\mathrm{f}-\sim 2 \mu \mathrm{g}$ crude bR prep pre-sucrose gradient ..........24

Figure 19. SDS gel plotted with migration distances from myosin, shown for both the mark 12 standard (left column) and bacteriorhodopsin remaining columns. .................................................24

Figure 20. Log-log plot of the relative molecular weight vs. migration distance from Myosin, Mark12

Figure 21. Post-centrifugation of PM in linear sucrose gradients. The PM band formed at a density of

Figure 22. Sucrose density and absorbance of $\mathrm{PM}$ as a function of fraction number for linear sucrose

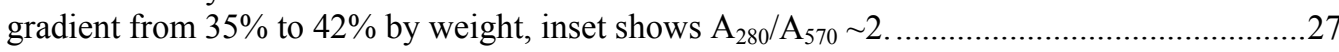

Figure 23. Sucrose density and absorbance of PM as a function of fraction number for linear sucrose

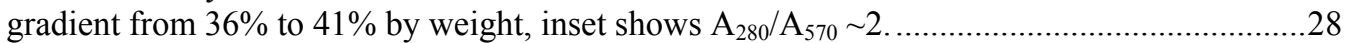

Figure 24. Sonicated PM in preformed linear sucrose gradient following ultracentrifugation....................31

Figure 25. Sonicated purple membrane distribution following equilibrium density gradient ultracentrifugation.

Figure 26. The distribution of bR following Sepharose gel filtration for a sample sonicated for 2 minutes at $30 \%$ power amplitude with a $0.2 / 2$ on/off pulse regime coupled with the non-sonicated sample.

Figure 27. The distribution of bR following Sepharose gel filtration for a sample sonicated for 6 minutes at $45 \%$ power amplitude with a $0.2 / 2$ seconds on/off pulse regime coupled with a non-sonicated sample. Note: the power amplitude total sonication time was increased but all other conditions were constant.

Figure 28. The distribution of bR following Sepharose gel filtration for a sample sonicated for 24 minutes at $45 \%$ power amplitude with a $0.2 / 2$ seconds on/off pulse regime and non-sonicated sample.. 35

Figure 29. \% solubilization of $b R$ at different concentrations of $\mathrm{OG}$ with respect to time...........................37

Figure 30. Calculated $\%$ active $\mathrm{bR}$ at different $\mathrm{OG}$ concentrations with respect to time. ...........................38

Figure 31. Pre-ultracentrifuge $\mathrm{A}_{280} / \mathrm{A}_{570}$ at $\mathrm{OG}$ concentrations from $20-100 \mathrm{mM}$ with respect to time.....38

Figure 32. Post-ultracentrifuge $\mathrm{A}_{280} / \mathrm{A}_{570}$ at $\mathrm{OG}$ concentrations from $20-100 \mathrm{mM}$ with respect to time...39

Figure 33. Percent solubilization of bR versus time at various $\mathrm{pH}$. 
Figure 34. Percent solubilization of bR versus $\mathrm{pH}$ at various times........................................................40

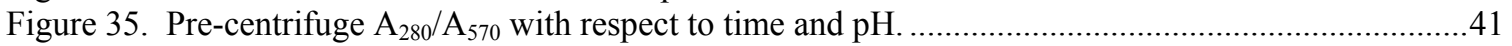

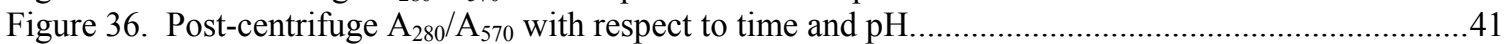

Figure 37. Biotin - HABA/Avidin standard curve. Change in absorbance at $500 \mathrm{~nm}$ of HABA/Avidin reagent with the addition of biotin. Biotinylated-bR is represented by the circled square symbols.

Figure 38. Absorbance of $b R$ and the emission spectrums of amber LEDs and red Laser pointer. ..............44

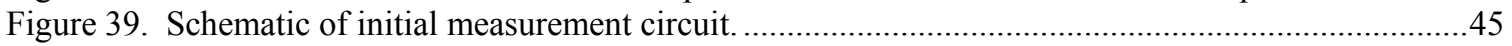

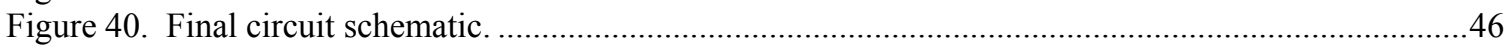

Figure 41. Representative photovoltaic response of bR sandwiched between ITO...................................47

Figure 42. Simplified setup of the photoelectric measurement of bR......................................................48

Figure 43. Photoresponse $(\sim 5 \mathrm{mV}, 11 \mathrm{x}$ amplification) of electrodeposited bR films with an ITO bottom

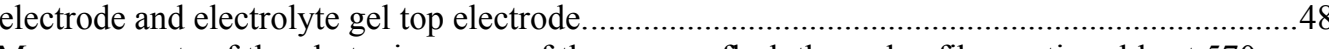

Figure 44. Measurements of the photonic power of the camera flash through a fiber optic cable at $570 \mathrm{~nm}$.

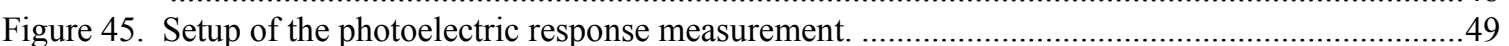

Figure 46. Current measurement of photon activated bR with gold sputtered top electrode measured with the SPA 4200.

Figure 47. Unamplified photovoltaic response of bR at various flash rates with an ITO bottom electrode and sputtered gold top electrode measured with the SPA 4200 ..............................................5

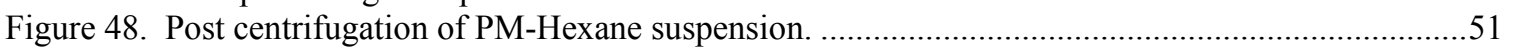

Figure 49. Trough area and transfer versus layer number. ........................................................................52

Figure 50. Photoelectrical response of 15 layers on an ITO substrate with a $5 \mathrm{~nm}$ thick gold top electrode

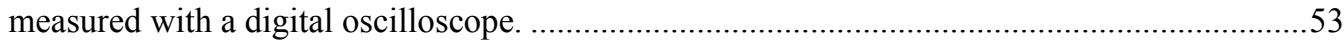

Figure 51. Photoelectrical response of 15 layers on an ITO substrate with a $5 \mathrm{~nm}$ thick gold top electrode

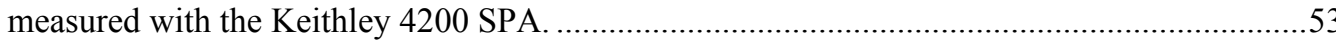

Figure 52. Parallel capacitance of dried bR versus time at four light intensities measured at $570 \mathrm{~nm}$ for the $10 \mu \mathrm{m}$ thick oriented sample (centered at light intensity of $0.0 \mathrm{~W} / \mathrm{m}^{2}$ ) ................................5

Figure 53. Parallel capacitance of dried bR versus time at four light intensities measured at $570 \mathrm{~nm}$ for the $22 \mu \mathrm{m}$ thick unoriented sample (centered at light intensity of $0.0 \mathrm{~W} / \mathrm{m}^{2}$ ) .............................56

Figure 54. Parallel resistance of dried bR versus time at four light intensities measured at $570 \mathrm{~nm}$ for the $10 \mu \mathrm{m}$ thick oriented sample (centered at light intensity of $0.0 \mathrm{~W} / \mathrm{m}^{2}$ ) ...............................56

Figure 55. Parallel resistance of dried bR versus time at four light intensities measured at $570 \mathrm{~nm}$ for the $22 \mu \mathrm{m}$ thick unoriented sample (centered at light intensity of $0.0 \mathrm{~W} / \mathrm{m}^{2}$ ) ..............................57

Figure 56. Change in resistance and capacitance versus light intensity measured at $570 \mathrm{~nm}$.....................57

Figure 57. RC time constant calculation based on LCR measurements. ....................................................58

Figure 58. Capacitance and resistance of dried bR versus frequency........................................................59

Figure 59. Capacitance per area versus voltage for $5 \mu \mathrm{m}$ and $10 \mu \mathrm{m}$ thick oriented samples and the $22 \mu \mathrm{m}$

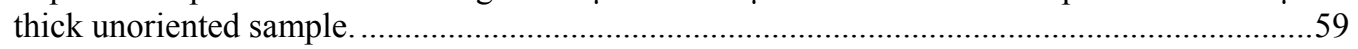

Figure 60. Capacitance per area versus average thickness for both oriented and unoriented samples.........60

Figure 61. Representative schematic model of dried films of $b R$, where $R_{\text {eqbR }}$ is the equivalent resistance of the dried bR film, $\mathrm{C}_{\mathrm{eqbR}}$ is the equivalent capacitance of the dried bR film, $\mathrm{h}$ is Planck's constant $\left(\mathrm{h}=6.626 \times 10^{-34} \mathrm{~J} \mathrm{~s}\right), \mathrm{c}$ is speed of light $\left(\mathrm{c}=3 \times 10^{8} \mathrm{~m} / \mathrm{s}\right)$, and $\lambda$ is wavelength in nanometers. ...60

Figure 62. Photovoltaic output of 3 samples with thickness of 2.8, 5. and $10 \mu \mathrm{m}$ at 4 light intensities

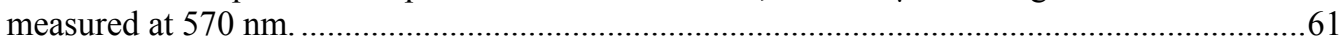

Figure 63. Photovoltaic responses of a $10 \mu \mathrm{m}$ thick sample at four different light intensities measured at $570 \mathrm{~nm}$

Figure 64. Photovoltaic responses of a $5 \mu \mathrm{m}$ thick sample at four different light intensities measured at 570 $\mathrm{nm}$

Figure 65. Photovoltaic responses of a $2.8 \mu \mathrm{m}$ thick sample at four different light intensities measured at $570 \mathrm{~nm}$

Figure 66. Surface plot of the light activated time constant of bR with respect to light intensity at $570 \mathrm{~nm}$ and thickness of bR sample. 
Figure 67. bR photocell layout.

Figure 68. Unamplified bR photoresponse to a $0.1 \mathrm{~Hz}$ excitation.........................................................68

Figure 69. Unamplified bR photoresponse to a $0.7 \mathrm{~Hz}$ excitation........................................................69

Figure 70. Unamplified bR photoresponse of single photocell with one and two lasers.........................69

Figure 71. Unamplified bR photoresponse with delayed activation of two lasers. ..................................70

Figure 72. Current versus time at flash rate of $0.5 \mathrm{~Hz}$ with and without a voltage bias applied across the bR photocell during the flashing. ........................................................................................ 71

Figure 73. Voltage versus time at a flash rate of $0.5 \mathrm{~Hz}$ with varying current biases. ..............................71

Figure 74. I-V characteristics of multi-monolayer bR structure with light activation and without.............72

Figure 75. Idealized I-V curve for a $\mathrm{p}-\mathrm{n}$ diode. ...............................................................................73

Figure 76. Hitachi FB-2000A focused ion beam system at Michigan Tech and schematic of general

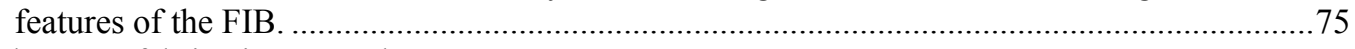

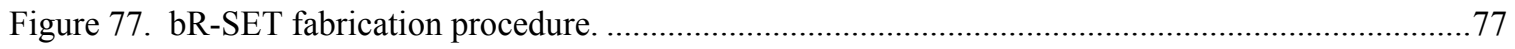

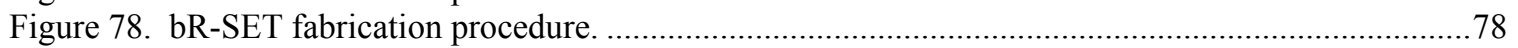

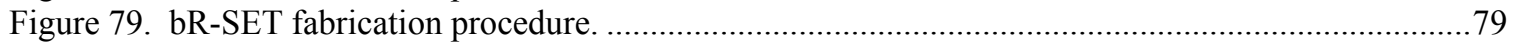

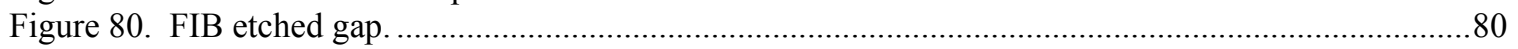

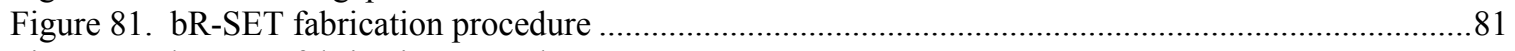

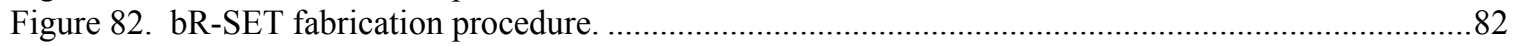

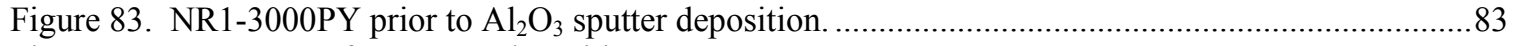

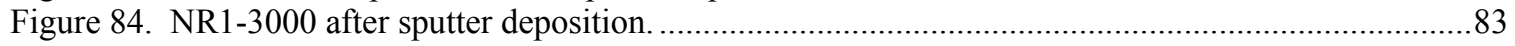

Figure 85. Substrate after cleaning with resist stripper. ..................................................................8 84

Figure 86. Substrate after cleaning with Pirahana (three parts hydrogen peroxide $30 \%$ to one part

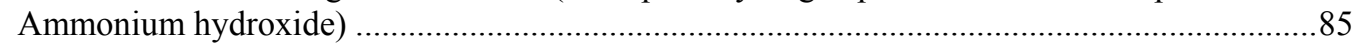

Figure 87. Measured I-V of $25 \mathrm{~nm}$ of sputter deposited $\mathrm{Cr}$.............................................................86

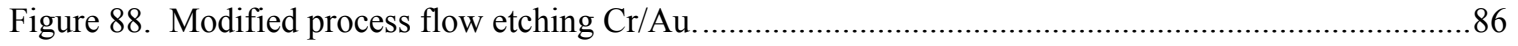

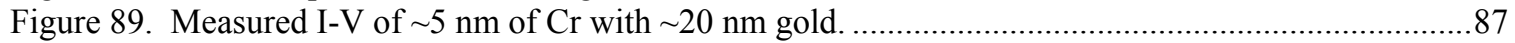

Figure 90. FIB etched perimeter is indicated by the gray lines in the shape of a square, using beam M1-500 in Fabrication Mode. The actual line width is $5 \mu \mathrm{m}$ and the size of the square is about $100 \mu \mathrm{m}$

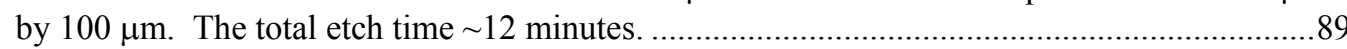

Figure 91. FIB etched perimeter is indicated by the gray lines in the shape of a square, using beam M1-500 in Fabrication Mode. The actual line width is $10 \mu \mathrm{m}$ and the size of the square is about $100 \mu \mathrm{m}$

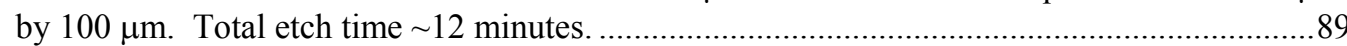

Figure 92. FIB etched perimeter is indicated by the gray lines in the shape of a square, using beam M1-500 in Fabrication Mode. The actual line width is $10 \mu \mathrm{m}$ and the size of the square is about $100 \mu \mathrm{m}$ by $100 \mu \mathrm{m}$. Total etch time $\sim 6$ minutes. . .90

Figure 93. FIB etched perimeter is indicated by the black lines in the shape of a square, using beam M1500 in Vector Scan Mode. The actual line width is $5 \mu \mathrm{m}$ and the size of the square is about 100 $\mu \mathrm{m}$ by $100 \mu \mathrm{m}$. Total etch time $\sim 1.5$ minute. ................................................................ 90

Figure 94. FIB etched perimeter is indicated by the black lines in the shape of a square, using beam M1500 in Vector Scan Mode. The actual line width is $5 \mu \mathrm{m}$ and the size of the square is about 100 $\mu \mathrm{m}$ by $100 \mu \mathrm{m}$. Total etch time $\sim 30$ seconds.

Figure 95. FIB etched perimeter is indicated by the black lines in the shape of a square, using beam M1500 in Vector Scan Mode. The actual line width is $5 \mu \mathrm{m}$ and the size of the square is about 100 $\mu \mathrm{m}$ by $100 \mu \mathrm{m}$. Total etch time $\sim 15$ seconds.

Figure 96. FIB deposited QDs, using beam M1-200 with an interlace of 8, and Dwell time of $9 \mu$ sec, beam current of $0.401 \mathrm{nA}$, and a depositon area of the $16.062 \mu \mathrm{m}^{2}$.

Figure 97. FIB deposited recipe for QDs, using beam M1-200 with an interlace of 8, and Dwell time of 9 $\mu \mathrm{sec}$, beam current of $2.33 \mathrm{nA}$, and a depositon area of the $16.062 \mu \mathrm{m}^{2}$......

Figure 98. Imaged at 110k (top) and 300k (bottom) with the FESEM image of tungsten QDs deposited using beam M1-200 with a beam current of $4.01 \mathrm{nA}$, interlace 4 and a dwell time varied from 4 $\mu \mathrm{s}$ to $11 \mu \mathrm{s}$. 
Figure 99. Imaged at 110k (top) and 300k (bottom) with the FESEM, image of tungsten QDs deposited using beam M1-200 with a beam current of $4.01 \mathrm{nA}$, interlace 8 and a dwell time varied from 3

$\mu$ s to $11 \mu \mathrm{s}$.

Figure 100. Imaged at 110k with the FESEM, image of tungsten QDs deposited using beam M1-100 with a beam current of $0.893 \mathrm{nA}$, interlace 4 and a dwell time varied from $4 \mu$ s to $11 \mu \mathrm{s}$.

Figure 101. Imaged at 110k (top) and 300k (bottom) with the FESEM, image of tungsten QDs deposited using beam M1-100 with a beam current of $0.893 \mathrm{nA}$, interlace 8 and a dwell time varied from $4 \mu \mathrm{s}$ to $11 \mu \mathrm{s}$.

Figure 102. Gap etched using M0-20 with an area of 32 and zoom of 8. The QDs were deposited using beam M1-100 with an interlace of 4 and dwell time of $8 \mu \mathrm{sec}$.

Figure 103. Gap etched using M0-20 with an area of $4 \mu \mathrm{m}^{2}$ and zoom of 2. The QDs were deposited using

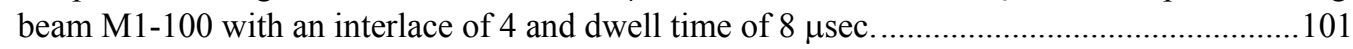

Figure 104. Representative schematic of a SET with bR integrated on the gate....................................103

Figure 105. Sensing platform, bR interfaced with a SET ...............................................................105

Figure 106. Current versus voltage measurements of a SET without bR immobilized on the gate with and without light.

Figure 107. Current versus time measurements of a SET without bR interfaced with the gate with and without light..................................................................................................... 106

Figure 108. Current versus time measurements of a SET with bR interfaced with the gate and flashed at

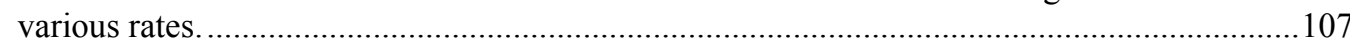

Figure 109. Current versus time measurements of a SET with bR interfaced with the gate and flashed at a flash rate of $0.4 \mathrm{~Hz}$..

Figure 110. Current versus time measurements of a SET with bR interfaced with the gate and flashed at a flash rate of $60 \mathrm{mHz}$.

Figure 111. Current versus time measurements of a SET with bR interfaced with the gate and flashed at a flash rate of $12 \mathrm{mHz}$.

Figure 112. I-V curve of SET coupled with bR.

Figure 113. I-T curves of SET coupled with $\mathrm{bR}$, bias with a source voltage of $-20 \mathrm{~V}$, and with a flashing light source at $134 \mathrm{~W} / \mathrm{m}^{2}$ (red) and $196 \mathrm{~W} / \mathrm{m}^{2}$ (blue).

Figure 114. I-T curves of SET coupled with $\mathrm{bR}$, bias with a source voltage of $0 \mathrm{~V}$, and with a flashing light source at $134 \mathrm{~W} / \mathrm{m}^{2}$ (red) and $196 \mathrm{~W} / \mathrm{m}^{2}$ (blue).

Figure 115. Change in current versus source bias voltage with a flashing light source at $134 \mathrm{~W} / \mathrm{m}^{2}$ (red) and $196 \mathrm{~W} / \mathrm{m}^{2}$ (blue)...... 


\section{LIST OF T ABLES}

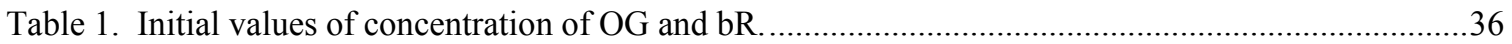

Table 2. Beam specifics for the FB-2000A FIB at Michigan Tech............................................................76

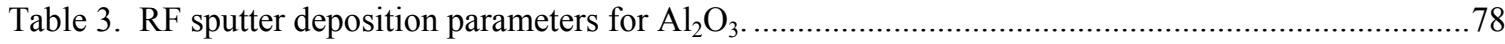

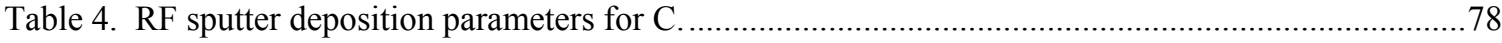

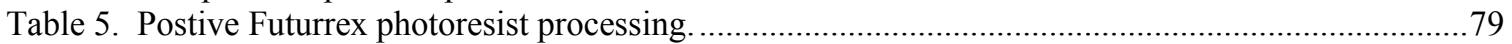

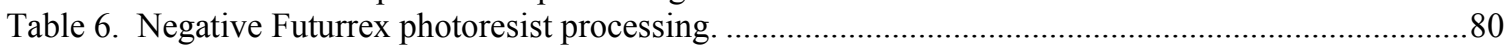

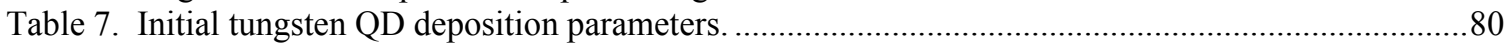

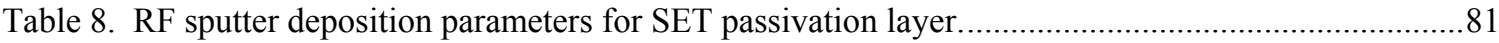

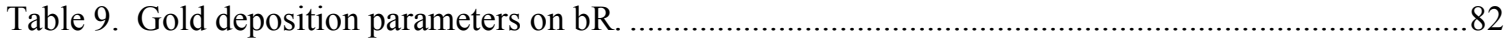




\section{Chapter 1 Introduction}

There is a growing role for biological materials to be incorporated into high technology applications. Using current processes, materials, and fabrication techniques for creating smaller, faster, denser and cheaper electronics may cease to be feasible from both a technological and economic standpoint within the next $10-15$ years [1]. Furthermore, the ability to identify environmental and security risks to human health requires improvements in sensor parameters such as size, response time, specificity, stability, simplicity, and power consumption. The use of biological materials to solve these challenges is currently being heavily researched. Along with those challenges researchers are also discovering more challenges including integrating biological and non-biological components, maximizing/optimizing response of a given amount of biomaterial, functionalizing biological components, and quantifying measurements. Innovative technologies must be developed to overcome these barriers.

Scientists in a variety of disciplines have worked to explore potential technological applications of biomolecular electronics, including their integration with semiconductorbased devices, such as transducers and photovoltaic cells. Vasevolodov [2] identified two definitions of biomolecular electronics. The first, and most broad definition, is the "science concerned with the processes in which electrons and electron currents are involved in biomolecules". The second definition, which is more applicable to this research, implies the "use of biomolecules or their complexes for application as independent functional devices capable of interfacing with modern electronic devices." Technological advancement, by the integration of semiconductor-based electronics and biomolecules will be harnessed to create bionanohybrid device and systems as shown schematically in Figure 1.

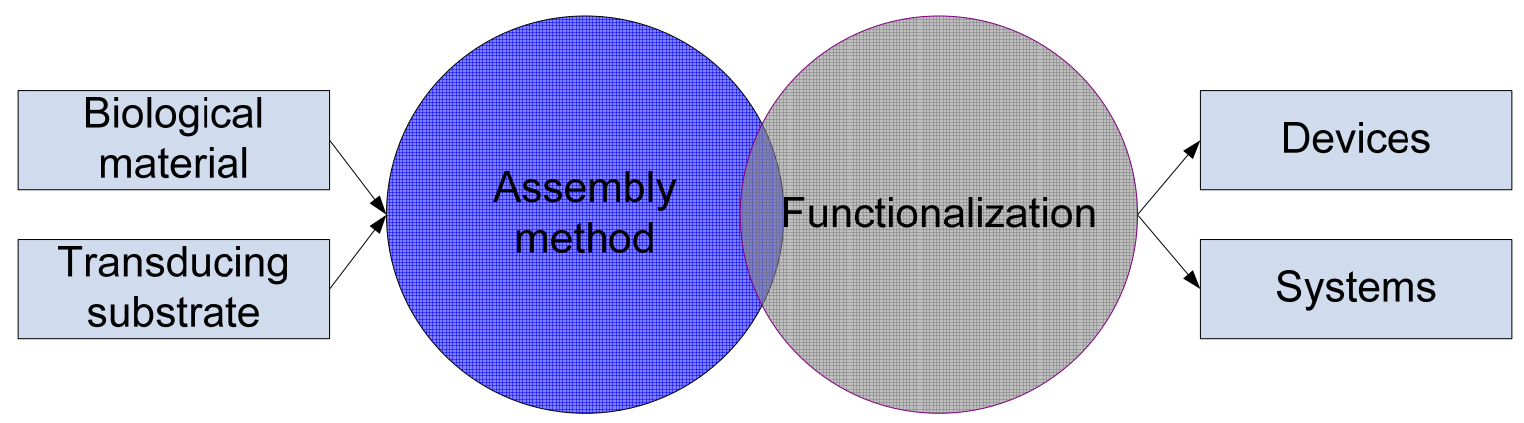

Figure 1. Process flow for development of a molecular electronic device.

One such biomolecule that could be interfaced with traditional electronics is bR. Bacteriorhodopsin contains photosynthetic complexes which transport/shift a proton, effectively acting as bioelectrical circuitry. Bacteriorhodopsin's photoelectrical 
properties and physical attributes suggest that it could provide the biomolecular platform for a toxin detecting photoelectric biosensor.

A possible layout for this sensor is shown in Figure 2. The ultraviolet-light emitting diodes (UV-LEDs) illuminates and activates semiconductor quantum dots (QDs) bound to $\mathrm{bR}$. Quantum dots are spherical with a diameter of $2-10 \mathrm{~nm}$ depending on the surface functionalization, desired emission wavelength, and material properties. When composed of cadmium and selenium, with a diameter of few nanometers they fluoresce within the visible spectrum in response to UV light. One method of controlling the light intensity emission of the QDs is through fluorescence-resonant-energy-transfer (FRET) coupling. FRET coupling quenches the light emission and instead causes nonradiative energy transfer [3] of the QDs energy. FRET coupling QDs with bR could potentially be used to control the photovoltaic response of bR [4].

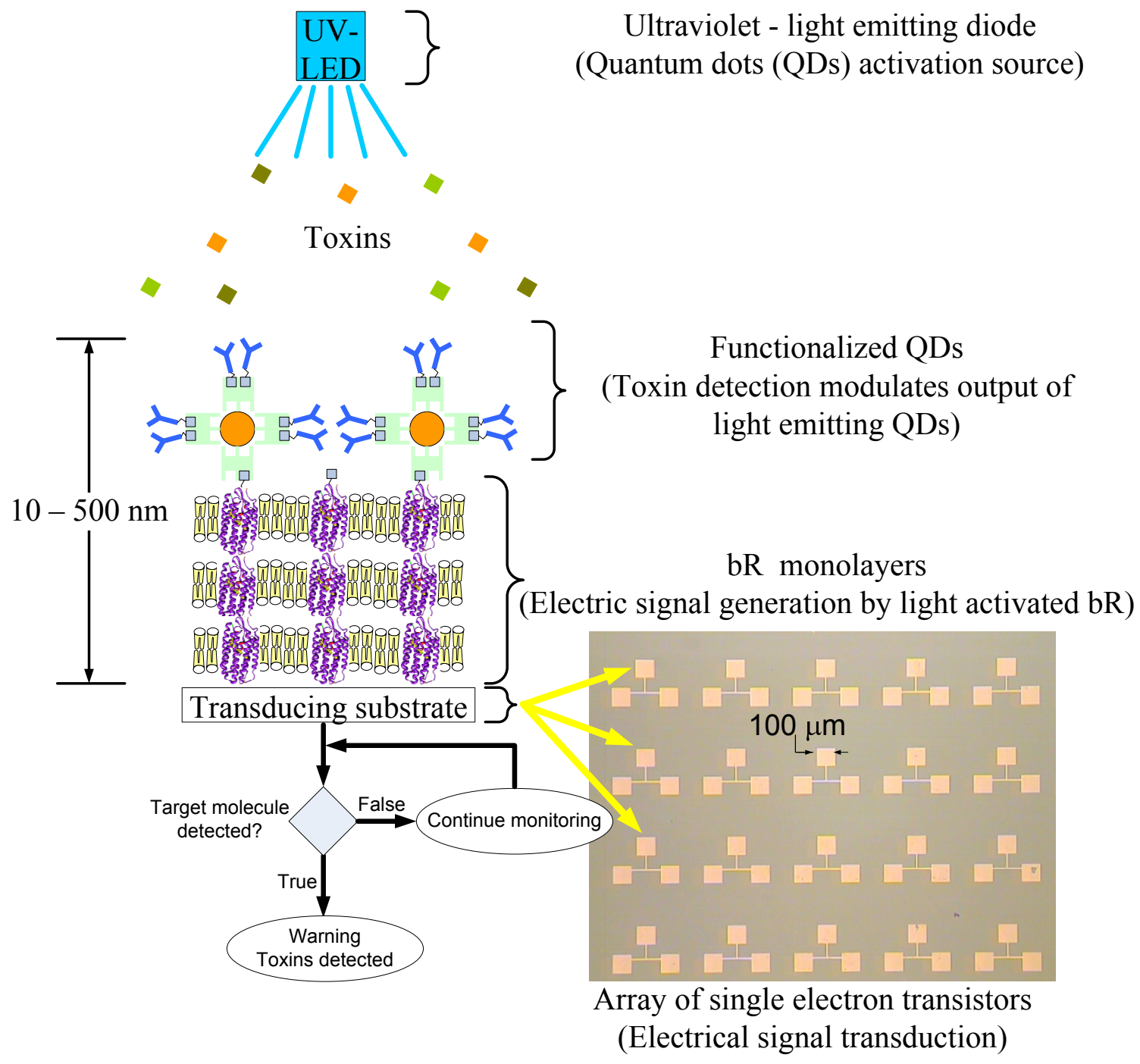

Figure 2. Schematic of possible toxin detection scheme. 
Taking advantage of these features, QDs could be used as the on-board light source to activate $b R$. By functionalizing QDs with a specific antibody, a specific antigen (e.g., plague, anthrax, ricin) would bind the antibody potentially altering the degree of FRET coupling or optical output of the QDs. The antibody-antigen binding event would modulate the electrical signal produced by the $\mathrm{bR}$ indicting the presence of a specific antigen. An ultra-sensitive toxin detecting biosensor could be created by exploiting the specific interactions that biological materials have with the outside environment. A bRbased biosensor might be ideal for environmental and national security applications because it would be nearly self-sufficient in terms of energy, potential individual molecule sensitivity, and small in size.

To create a toxin detecting biosensor, bR must be immobilized and deposited on a transducing device such as MOSFET or SET. The proposed sensor format presents a number of scientific challenges as shown in Figure 3, and briefly described earlier.

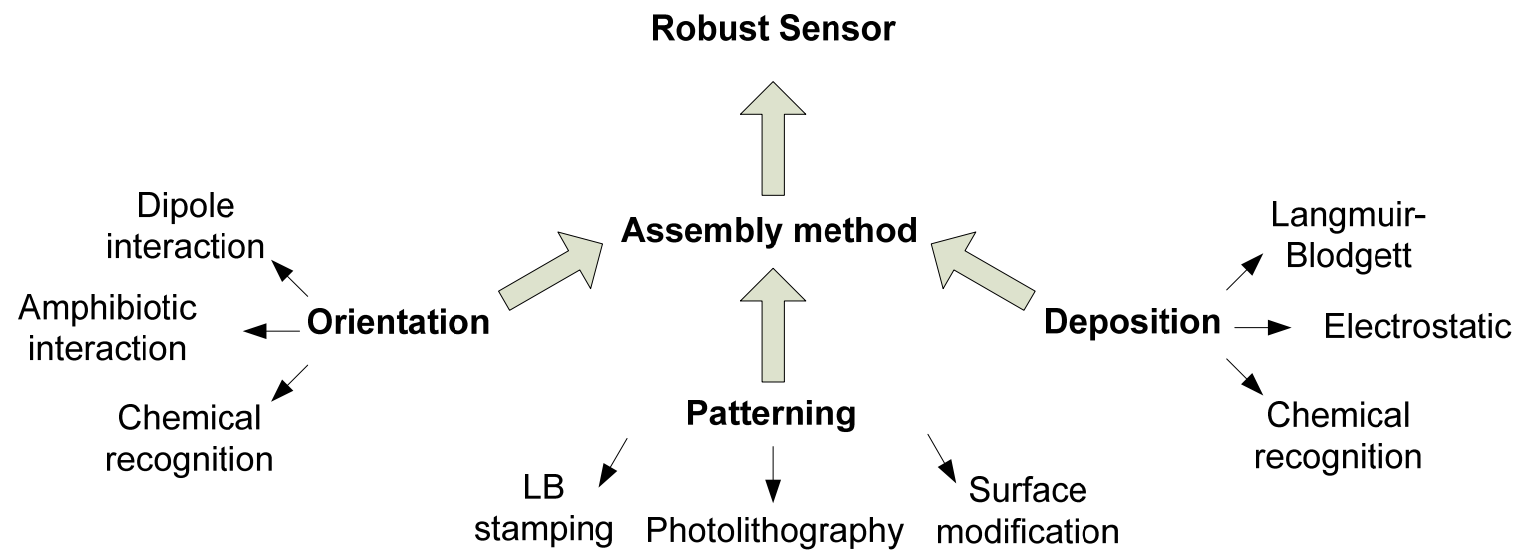

Figure 3. Proposed bR biosensor assembly techniques.

This research will focus on one of these challenges: integrating bR with a SET, then developing a method for immobilizing multiple bR monolayers on a SET, and measuring and modeling the photoelectric response of the bionanohybrid device. The completion of this work will lay the foundation for the toxin detecting photoelectric biosensor, and lead to the integration of the toxin detecting agents and bR activation layers as shown in Figure 2. 


\section{Chapter 2 Literature Review}

To determine the current state-of-art associated with SETs, MOSFET-bR devices, and orienting, depositing, and patterning bacteriorhodopsin, a literature review was conducted.

\subsection{Overview of Halobacterium, Purple Membrane, and Bacteriorhodopsin}

Scientists believe that life on Earth began more than 3.5 billion years ago [5] with prokaryotic cells, while the earth was still forming its atmosphere, oceans, and lands. To survive, these organisms evolved and adapted rapidly to exist in extraordinary environments such as extreme heat, cold, salty, acidic, or alkaline conditions. Prokaryotes are generally single-celled organisms which do not have a nucleus. They are divided into two domains, bacteria and archaea. A subgroup of archaea called extreme halophiles (salt-loving) contains a bacterium called Halobacterium halobium, also known as Halobacterium salinarum. Halobacterium salinarum can be found in nature in extreme salinity environments (e.g. San Francisco saltterns, Dead sea). This organism produces both red and purple membranes. To survive in anaerobic conditions of extreme salinity environments the bacterium over-produces purple membrane when exposed to sunlight. The purple membrane is a transmembrane protein composed of both bacteriorhodopsin and phospholipid with a weight ratio of $\sim 3: 1$, respectively. Due to the anaerobic environment, adenosine triphosphate (ATP) - the energy currency in cells cannot be generated through oxidative phosphorylation due to low concentrations of oxygen in the extremely salty solution [6]. In this case bacteriorhodopsin converts light energy into chemical energy by pumping a proton out of the cell thus creating a charge gradient across the cell wall. The proton gradient drives a proton back into the cell through a membrane ATP synthase complex shown in the Figure 4, similar to mitochondrion and chloroplast [7]. This method of ATP generation is also known as photophosphorylation. 


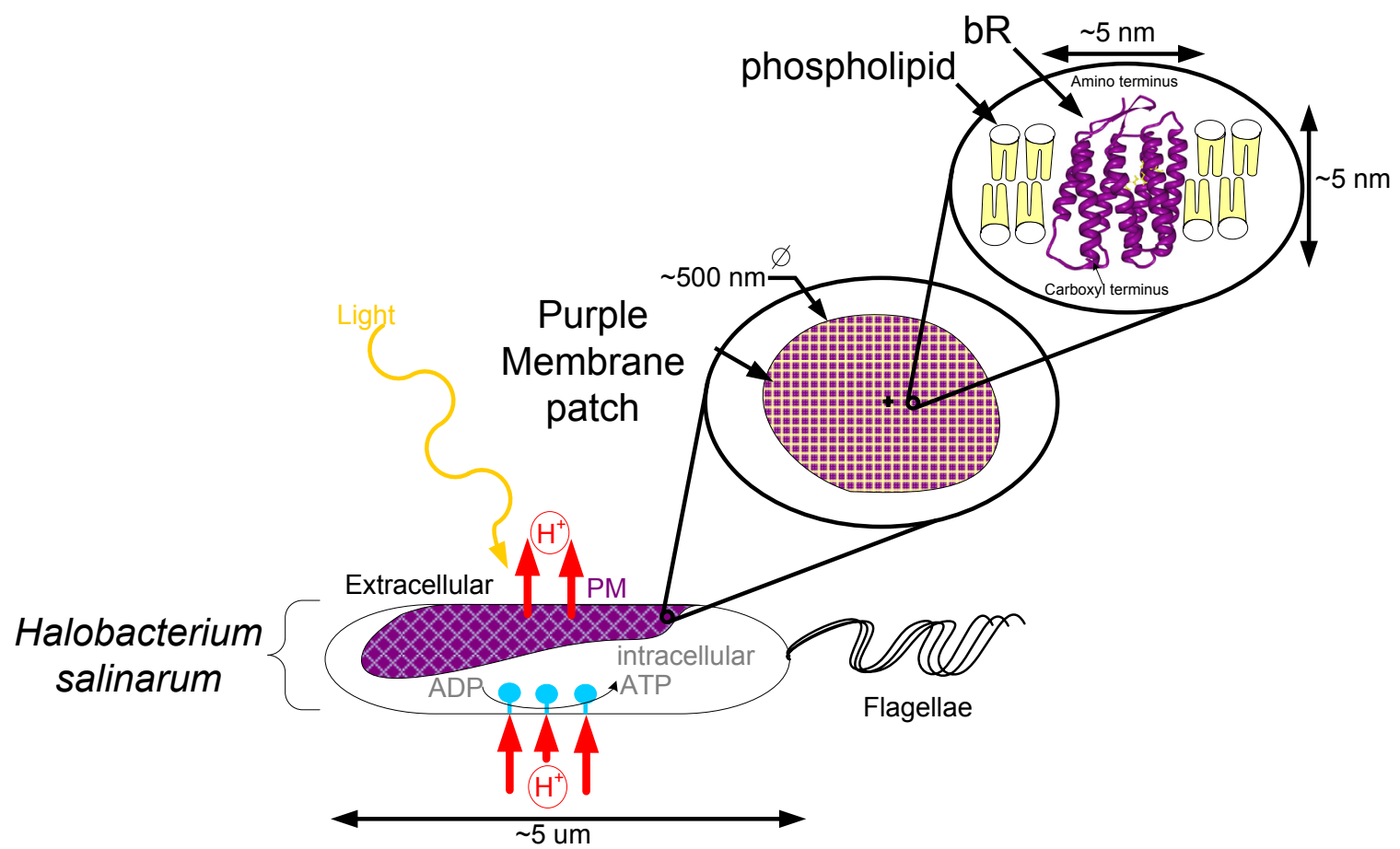

Figure 4. Depiction of proton pumping and ATP synthesis in Halobacterium salinarum.

Activation of bR in the 460-640nm photonic range results in a trans-cis isomerization of the $\mathrm{bR}$ retinal and induces a proton pumping/shifting mechanism. This proton movement within the bR produces a voltage potential and current. The voltage potential increases with increasing photonic intensity.

This protein is robust, it can function wet and dry and can withstand extreme environmental conditions. The name "bacteriorhodopsin" originates from its presence in the bacteria Halobacterium salinarum, and it is structurally similar to the visual rhodopsins, which enable mollusks, arthropods, and vertebrates to resolve images [2] and is one of the reasons why bR is heavily researched.

Many of the intrinsic properties of bR suggest it would be an outstanding candidate for use in hybrid, bionanoelectronic devices [6]. The protein possesses long-term stability with respect to heat $\left(140^{\circ} \mathrm{C}\right)$ and cold $\left(-190^{\circ} \mathrm{C}\right)$ [8], wet and dry environments, acid and alkali solutions, and photochemical degradation [9]. Methods for depositing monolayers include Langmuir-Blodgett techniques, biotin-streptavidin linking, electrostatic layering, electrophoretic deposition, and antigen-antibody linking. A monolayer of bR has the potential to generate a photoelectric response as large as $300 \mathrm{mV}$ [10] and as quickly as 5 picoseconds after illumination. This is one reason why monolayers of $b R$ are attractive to researchers.

Hampp [10] helped clarify the understanding of bacteriorhodopsin by compiling the known structural and physiochemical properties of the PM, which contains bR. It has been noted that when water is removed, the absorption and photochemical activity of 
bacteriorhodopsin is maintained. Bacteriorhodopsin is also stable in most non-polar organic solvents such as hexane.

PM physical properties include:

$>\mathrm{PM}$ consists of lipids and $\mathrm{bR}$ at a molar ratio of about 10:1;

$>\mathrm{PM}$ is a two dimensional, hexagonal crystalline array of bR amino acid chains (i.e., trimers) which are uniformly oriented;

$>$ Molecular weight of $\mathrm{bR} \sim 26,000 \mathrm{Da}$;

$>$ Density $\sim 1.18 \mathrm{~g} / \mathrm{cm}^{3}$;

$>$ Refractive index $\mathrm{n}_{\mathrm{PM}} \sim 1.45-1.55$;

$>$ PM patches are irregularly shaped;

$>$ Lateral dimensions up to $5 \mu \mathrm{m}$;

$>$ Constant thickness of $\sim 5 \mathrm{~nm}$;

$>$ Dielectric constant depends on bR concentration, humidity, ion concentration, and composition of layers (e.g. percent lipid to protein);

bR's stability and characteristics:

Air dried/immobilized $~$ years;

$>$ Temperatures $<80^{\circ} \mathrm{C}$ (in solution) and $<140^{\circ} \mathrm{C}$ (dry);

$>\mathrm{pH}: \sim 1$ to 12 ;

$>$ Isoelectric point (IEP) 5.4;

$>$ Ionic strength $(3 \mathrm{M} \mathrm{NaCl})$;

$>$ Proteases digestion;

$>$ Proton pump;

$>$ Functions wet and dry;

bR's photoelectric response depends on;

$>$ Light intensity;

$>$ Activation wavelength;

$>$ Activation angle;

$>$ Number of layers deposited;

$>$ Wet or dry films;

$>$ External electric fields;

$>$ Humidity;

$>$ Temperature;

$>$ Orientation.

Many of the intrinsic properties of PM can be utilized to control or modulate photoelectric responses of $\mathrm{bR}$. So, attention to detail is important when taking measurements.

Bacteriorhodopsin is a dipolar molecule with net negative charge when the $\mathrm{pH}$ is greater than 5 [11]. Birge [6] indicates the charge density to be -3 to -4 electron units in a neutral $\mathrm{pH}$ solution. In a range of $\mathrm{pH}$ from $5-12$ the intracellular side is more negative than the extracellular side as previously shown in Figure 4, which is most likely from the 
deprotonation of a $\mathrm{COOH}$ group on the intracellular side. The charge density on the intracellular side and extracellular side of the $\mathrm{bR}$ can be modified by changing the $\mathrm{pH}$ of the aqueous PM solution but the exact changes are debatable [11, 12]. Controlling the surface charge density is a critical tool for orienting and depositing bR (e.g. Ionic self assembly, electrophoretic deposition, Langmuir-Blodgett), these methods will be discussed later.

Understanding and optimizing these physiochemical properties will enable our research to achieve maximum photoelectric output through the development of a method to immobilize bR on a SET.

The research associated with this project builds on contributions leading to three Nobel Prizes in chemistry - Mitchell's development of chemiosmotic theory, the efforts of Deisenhofer, Huber, and Michel in defining the structure of bacteriorhodopsin, and the Langmuir trough, see Figure 5. Although this project incorporates a number of other contributions from the scientific community, it is interesting to note that background research has been so highly regarded.

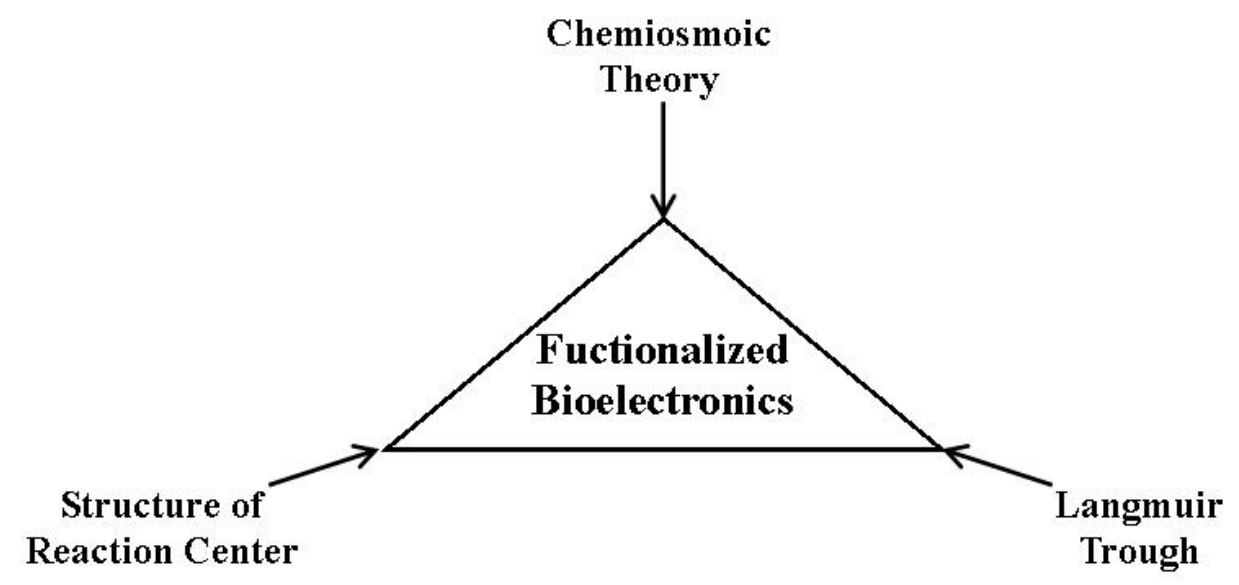

Figure 5. Nobel contributions integrated into this project.

\subsection{Bioelectronics}

In 1978, Peter Mitchell was awarded the Nobel prize "for his contribution to the understanding of biological energy transfer through the formulation of the chemiosmotic theory" [13]. Mitchell hypothesized that it is an electrochemical proton gradient across cell membranes that causes ATP to be synthesized, opposed to earlier hypotheses involving direct chemical interaction within cellular membranes between oxidizing and phosphorylating enzymes [14]. A difference in electric potential and $\mathrm{pH}$ (i.e., hydrogen ion concentration) constitute Mitchell's 'protonmotive force' in the gradient. The translocation of protons across $5 \mathrm{~nm}$, from the cytoplasmic side to the extracellular side of the membrane, is driven by electron flow through a series of electron and proton 
carriers. Mitchell's chemiosmotic theory is likely "the best available model to study an ion pump" [15].

A decade later, in 1988, Johann Deisenhofer, Robert Huber, and Hartmut Michel were jointly awarded the Nobel Prize in chemistry "for the determination of the threedimensional structure of a photosynthetic reaction center" [16]. Michel developed the methodology to obtain highly ordered crystals of Rhodopseudomonas virdis, which enabled the three 1988 awardees to determine the structure of its photosynthetic reaction center. This discovery clarified how electrons can be transferred in biological systems and also helped elucidate the first complete 3-dimensional structure of bacteriorhodopsin.

For a $\mathrm{bR}$ based bionanoeletronic device to obtain the highest electrical response, the $\mathrm{bR}$ molecules need to be unidirectionally oriented. A number of methods have been used to orient biological molecules. These orientation methods can be broken into two main categories: electric field assisted or non-electric field assisted. The following section describes the specific methods to assemble highly oriented layers of bR; LangmuirBlodgett [12, 17, 18]; Langmuir-Blodgett with electric field assisted deposition [19, 20]; electric field sedimentation [21, 22]; chemical molecular recognition [23, 24]; and electrolyte layering [12].

\subsection{Deposition and Immobilization Methods}

\subsubsection{Langmuir-Blodgett}

In 1932, Irving Langmuir was awarded the Nobel Prize "for his discoveries and investigations in surface chemistry" [13]. One of his contributions to surface science was the development of a device to create a layer of homogeneous, densely-packed molecules. The molecules which Langmuir studied using this device were amphiphilic, possessing both hydrophilic and hydrophobic components. The Langmuir trough is simple and robust. It consists of a basin filled with purified water (the subphase), a fixed end, and a moveable end [25]. The molecules are suspended in an organic solvent and the solution is spread on top of the subphase in the trough, typically using a syringe. As the solvent evaporates, the amphiphilic molecules spread and position themselves at the air/water interface - the hydrophilic component remains in contact with the water with the hydrophobic component in the air. The moveable ends of the Langmuir trough (the barriers) are used to decrease the surface area which causes the amphiphilic molecules at the surface to become more densely packed and oriented. To deposit uniform monolayers of constant thickness and density, the surface pressure and deposition speed must be optimized. The barrier serves not only to pack the molecules on the subphase surface, but also to maintain pressure on the film as it adheres to a solid substrate being drawn perpendicularly out of the subphase. Adhesion of the monolayer to the substrate is caused by the subphase solution draining between the monolayer and substrate [26]. There are several different deposition configurations, the most common three are Z-type, 
X-type, and Y-type, as shown in Figure 6. A Z-type deposition occurs on the upward stroke on hydrophilic substrates while the X-type deposits on the downward stroke of hydrophobic substrates. A Y-type has monolayers deposited on the upward and downward stroke [26].
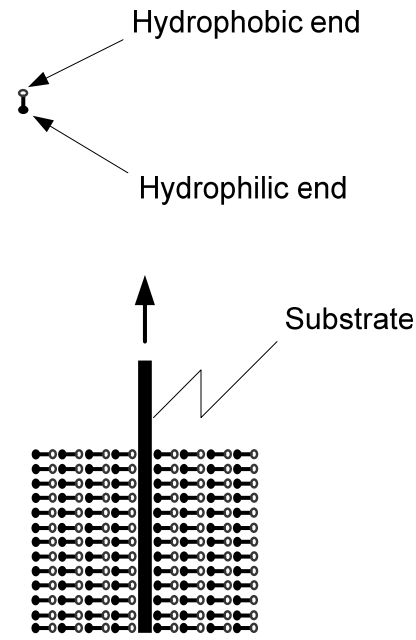

Z-type on a hydrophilic substrate
Direction of substrate during deposition

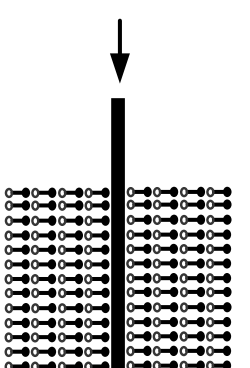

X-type on a hydrophobic substrate

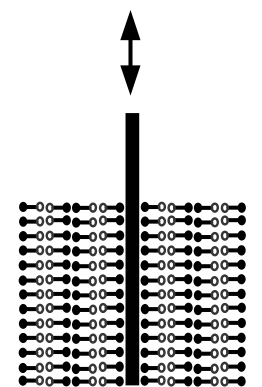

Y-type on a hydrophilic substrate

Figure 6. Idealized deposition of amphiphilic monolayers with different deposition arrangements.

This device, and the associated film, is commonly referred to as a Langmuir-Blodgett (LB) trough, and film, acknowledging Katherine Blodgett's contribution to surface science - having provided the first detailed description of sequential monolayer transfer [27]. The LB method is an effective technique for orientating and depositing amphiphilic molecules. According to Maximychev et al. [28], the orientation of the bR molecule at the air/water interface can be controlled, however, experimental measurements of the orientation have been inconclusive. Since bR is a dipolar molecule Theoretically, at a $\mathrm{pH}$ $>5.4$, the cytoplasmic side of the bR molecule preferentially orients on the subphase, whereas at $\mathrm{pH}<5$ the extracellular side preferentially orients.

The Langmuir-Blodgett technique has been utilized for years to deposit monolayers of purple membrane. Hwang et al. [17, 29] provided the first detailed description of LB monolayers of purple membrane and the associated photoresponse in a dry cell. Their method of preparing bR for spreading onto a subphase is widely accepted and still in use today $[18,30-32]$.

With the LB method, Hwang et al. [17, 29], Ikonen et al. [33] and Chio et al. [34], showed it was possible to deposit $1-60 \mathrm{z}$-type monolayers of bR or bR soya-PC. Chio et al. [34] found that the optimal number of depositions is 10 with a subphase of $\mathrm{pH} 10$. After each monolayer deposition, the bR was air dried for 20 minutes. Following the completion of the multi-monolayer suprastructure, a second electrode was applied either by sputtering (e.g., palladium, gold) or by an ITO slide or Indium Gallium (InGa) lowmelt alloy as the top electrode, created a bR photocell. Initially it was suggested that 
$85 \%$ of the bR was oriented [35] but later studies suggested that the orientation of monolayers formed at the interface of a neutral $\mathrm{pH}$ subphase are somewhat random [36].

Deposition and immobilization of monolayers of bR was not a trivial task. It has been shown that molecule re-arrangement may occur during or shortly after deposition [26]. The possible mixed-bR orientation may occur at the subphase and/or possibly during deposition. To overcome the possible mixed-orientation at the subphase, and possibly during deposition, Langmuir-Schaefer (LS) techniques, electric field assisted, magnetic field assisted, electrostatic layer-by-layer assembly, or conjugation of molecules have been investigated with limited success.

Nicolini et al. [19] suggested that an electric field from a two-electrode system, one in the subphase and the other a few millimeters above the subphase, could be used to increase the orientation of a film of bR molecules in an LB trough, as shown in Figure 7. These researchers deposited $\mathrm{bR}$ on filter paper with $0.10 \mu \mathrm{m}$ pore size, and measured the photoresponse of the monolayer by placing it between two electrolyte solutions. They determined that the electric field increased the orientation of the monolayers by an ON and OFF comparative test. With the electric field ON, the measured photoresponse increased $\sim 80 \mathrm{X}$ when compared to the standard deposition method. With all other factors being equal, they concluded that the output was directly related to the degree of orientation with a higher degree of uniform orientation giving a larger output. They did not describe the type of deposition or verify the repeatability of the experiments. Later, using the method Nicolini developed, Bertoncell et al. [20] deposited monolayers of bR on ITO glass with the Langmuir-Schaefer (LS) method, a horizontal deposition method that used an electric field to assist in the orientation of the bR monolayers at the air/water interface, and a similar measurement setup. A larger electrical output suggested the orientation was improved but it was limited to substrates with a roughness approximately the thickness of a monolayer and they did not mention the repeatability of the results.

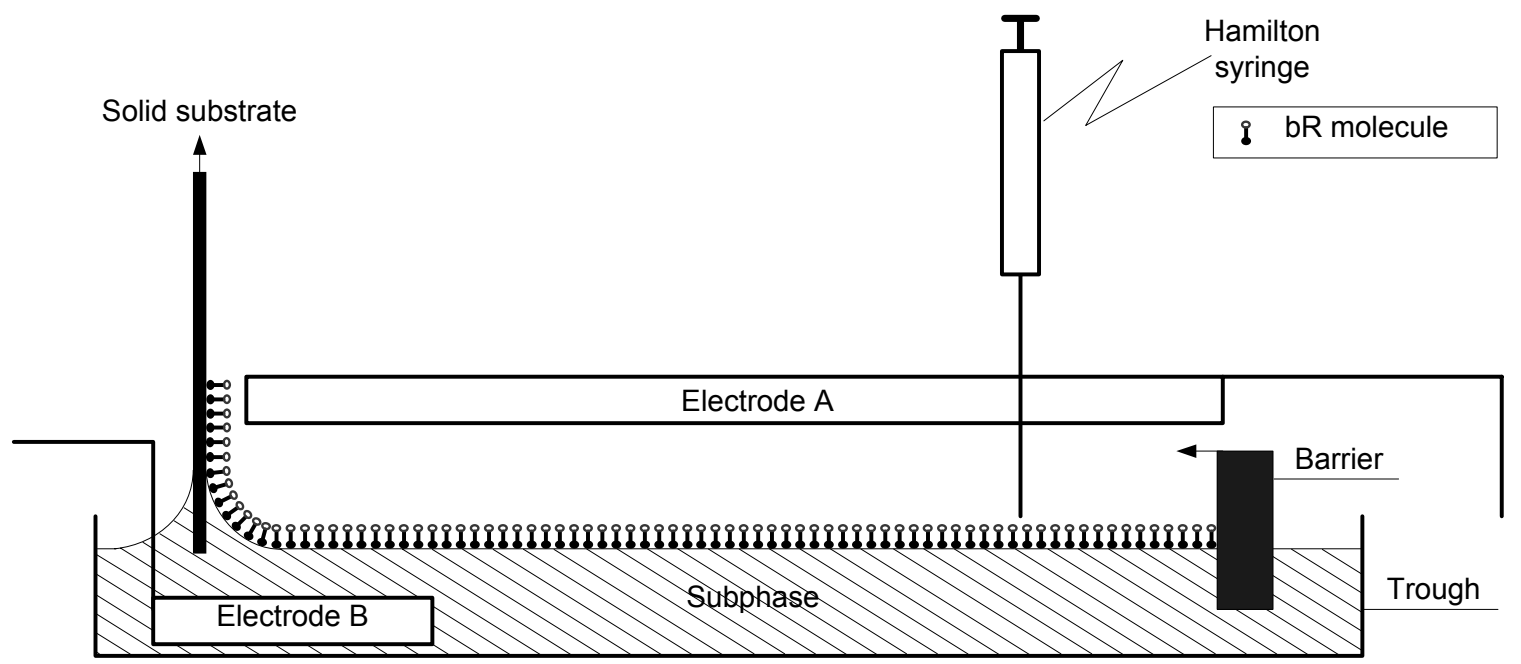

Figure 7. LB deposition of bR onto a solid substrate in the presence of an electric field. The polarity of the electrodes A and B control the bR orientation. 
Other methods of depositing oriented monolayers with the Langmuir-Blodgett trough include the horizontal deposition also called the Langmuir-Schaefer (LS) method [37]. In this case, the substrate is held horizontal, lowered towards the subphase, and the hydrophobic end of the floating monolayer attaches to the substrate surface as shown in Figure 8 [26].

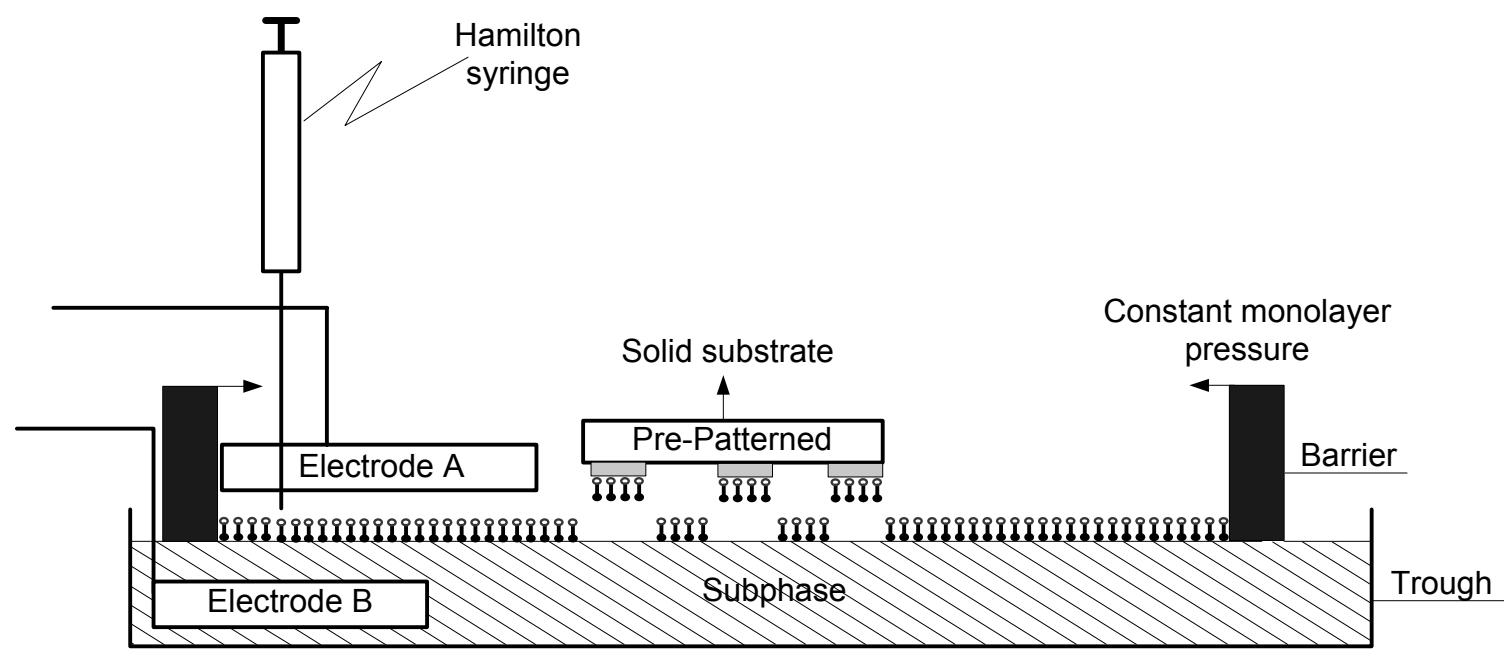

Figure 8. Deposition of bR using Langmuir-Schafer method.

Miyaska and Koyama [38] deposited multiple layers of bR with a slightly modified horizontal deposition technique [39]. As shown in Figure 9, an ITO substrate is slightly tilted $<5^{\circ}$ from horizontal. The first LS monolayer is deposited as the hydrophobic side of the molecule contacts the air/water interface and the second monolayer is then deposited on the up-stroke by maintaining the surface pressure of $20 \mathrm{mN} \mathrm{m}^{-1}$ on the monolayer at the air/water interface.

The photoresponse was measured in an electro-chemical cell containing $0.1 \mathrm{M} \mathrm{KCl}$. The multilayer PM deposited on $\mathrm{SnO}_{2}$ coated glass was the working electrode, a platinum wire was the counter-electrode, and a saturated Calomel electrode was the reference. With this method the researchers achieved a quantum conversion efficiency of 0.01 from six monolayers of deposited PM. The measured photocurrent was $>60 \mathrm{nA} \mathrm{cm}^{-2}$ from an incident photon flux of $4 \times 10^{15}$ photon $\mathrm{cm}^{-2}$.

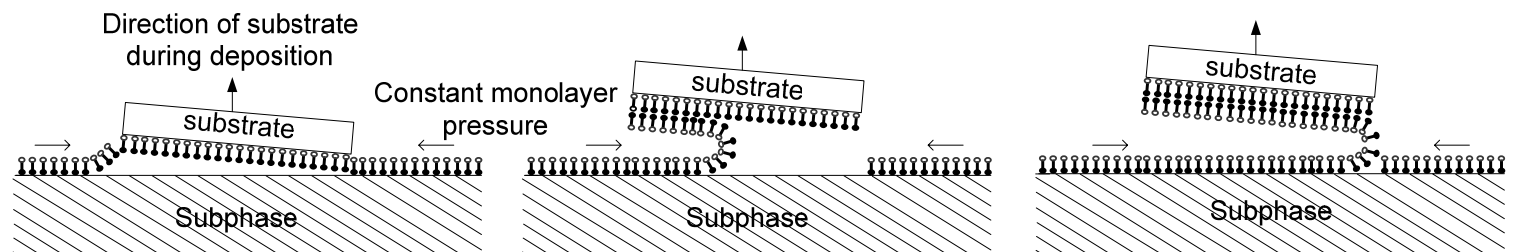

Figure 9. Sequence of modified horizontal deposition technique. 
By combining the LS technique and the addition of an electric field applied across the air/water interface, researchers [20,40] have been able to produce monolayers of more uniformly oriented bR based on the output signal, but these results were based on electrical output and not visual inspection of the monolayers. For example, these results do not indicate monolayer stacking for a single deposition which could result in increased electrical output.

\subsubsection{Electrostatic Layering}

Since bR is a dipole molecule, ionic attraction between oppositely charged sides can be used to deposit and orient purple membrane as shown by Fisher et al. [41]. In He et al. [42], multilayers were achieved through the absorption techniques by alternating layers of polycationic poly(dimethyldiallylammonium chloride) (PDAC) and purple membrane. These layers were deposited on a substrate treated in an ultrasonic bath at $25{ }^{\circ} \mathrm{C}$ in a mixture of ethanol/acetone/chloroform $(2 / 1 / 1, \mathrm{v} / \mathrm{v} / \mathrm{v})$ and then again in a $2 \% \mathrm{KOH}$ aqueous solution. This left the substrate with a net negative charge. The treated substrate was either dried and used immediately or stored in DDI water. The treated substrate was then incubated for ten minutes in either the PM or PDAC solution, depending on polarity, as shown in Figure 10. In the multi-monolayer structure shown in Figure 10, each layer was washed in DDI water and dried with nitrogen prior to depositing the next monolayer. Although this method was simple to implement the degree of orientation of the $b R$ monolayers and the number of monolayers deposited was difficult to predict, and the maximum number of monolayers that can be deposited was $<12$ [42].

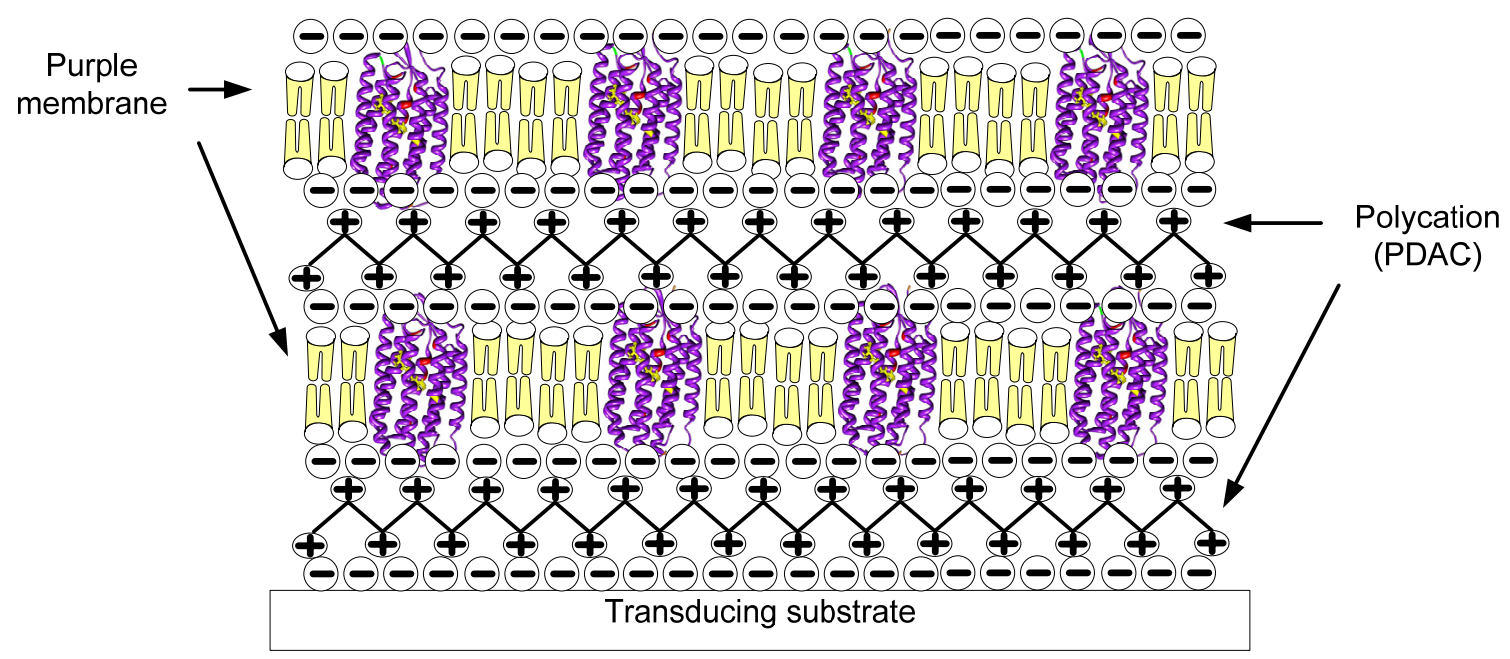

Figure 10. Scheme of PDAC/PM layering on negatively charged substrate.

\subsubsection{Electrophoretic Deposition}

When an aqueous solution containing bR has a $\mathrm{pH}$ in the range of $5-12$, the bR will orient and deposit, when the placed between conductive plates and a voltage is applied, as shown in Figure 11. The electric field between the plates causes the cytoplasmic side 
(the more negatively charged side) to electrophoretically move and deposit on the positive electrode [12]. This method is simple to perform and the orientation of the $b R$ can be effectively controlled, but there is not much control of the number of layers of $b R$ molecules deposited. Birge [6] suggests that this method is good if a relatively thick layer of bR (i.e., 1000's of monolayers) is required.
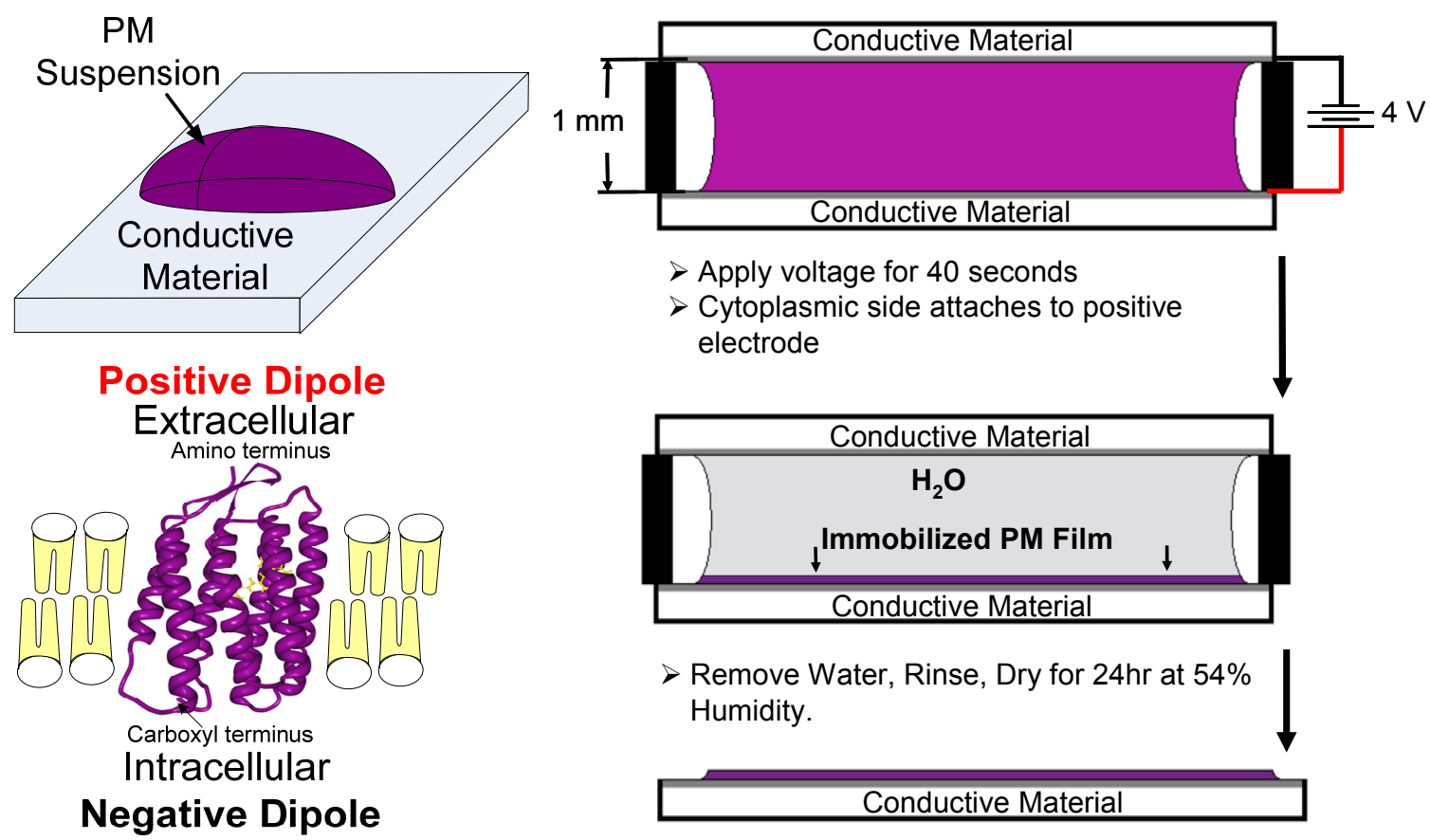

Figure 11. Schematic representation of electrophoretic deposition and image of $b R$ in phospholipid matrix.

\subsubsection{Selective Recognition Deposition}

Monolayer formation can also be achieved by using the binding interaction between ligands and proteins, such as biotin [43] and streptavidin [44]. Streptavidin, like avidin found in egg whites, has a molecular weight of $\sim 53,000 \mathrm{Da}$, is stable over a large $\mathrm{pH}$ range, is soluble in aqueous solutions, has an isoelectric point of 10 and is stable over a large temperature range. Streptavidin and avidin are glycoproteins containing four identical subunits that serve as binding sites for biotin. Biotin, known as vitamin $\mathrm{H}$ or $\mathrm{B}_{7}$, has a molecular weight of $\sim 244 \mathrm{Da}$ and binds very strongly to the Streptavidin molecule. The biotin-streptavidin interaction creates one of the strongest non-covalent bonds in nature $\left(\mathrm{Ka} \sim 10^{15} \mathrm{M}^{-1}\right)$.

By using the biotinylation process $[23,45]$ biotin can be specifically attached to $\mathrm{bR}$ at lysine group 129 at a $\mathrm{pH} \sim 9+/-0.3$ (shown in Figure 12). Biotinylated bR and streptavidin will form a self-assembling, self-orientating, stable near-covalent linkage. The biotin-streptavidin binding is essentially irreversibly bound to the solid support by the nearly covalent binding of a self-assembling monolayer of biotin functionalized with 
the thiol ligand on the gold surface as shown in Figure 12. Once bound, biotinstreptavidin forms a stable linkage which is unaffected by the most extreme $\mathrm{pH}$, organic solvents, and other denaturing agents, making this a stable linkage [46].

However, random orientation of the proceeding layers might result from nonspecific biotinylation of the bacteriorhodopsin and excess binding of biotin to the $b R$, which might eliminate or reduce the functionality of the bacteriorhodopsin. Another possible drawback to this method for layering is that the density of the layer is dependent on the incubation duration (i.e., the longer the incubation time up to 4 hours the denser the layer). More information about this process can be found in [44, 47-50].
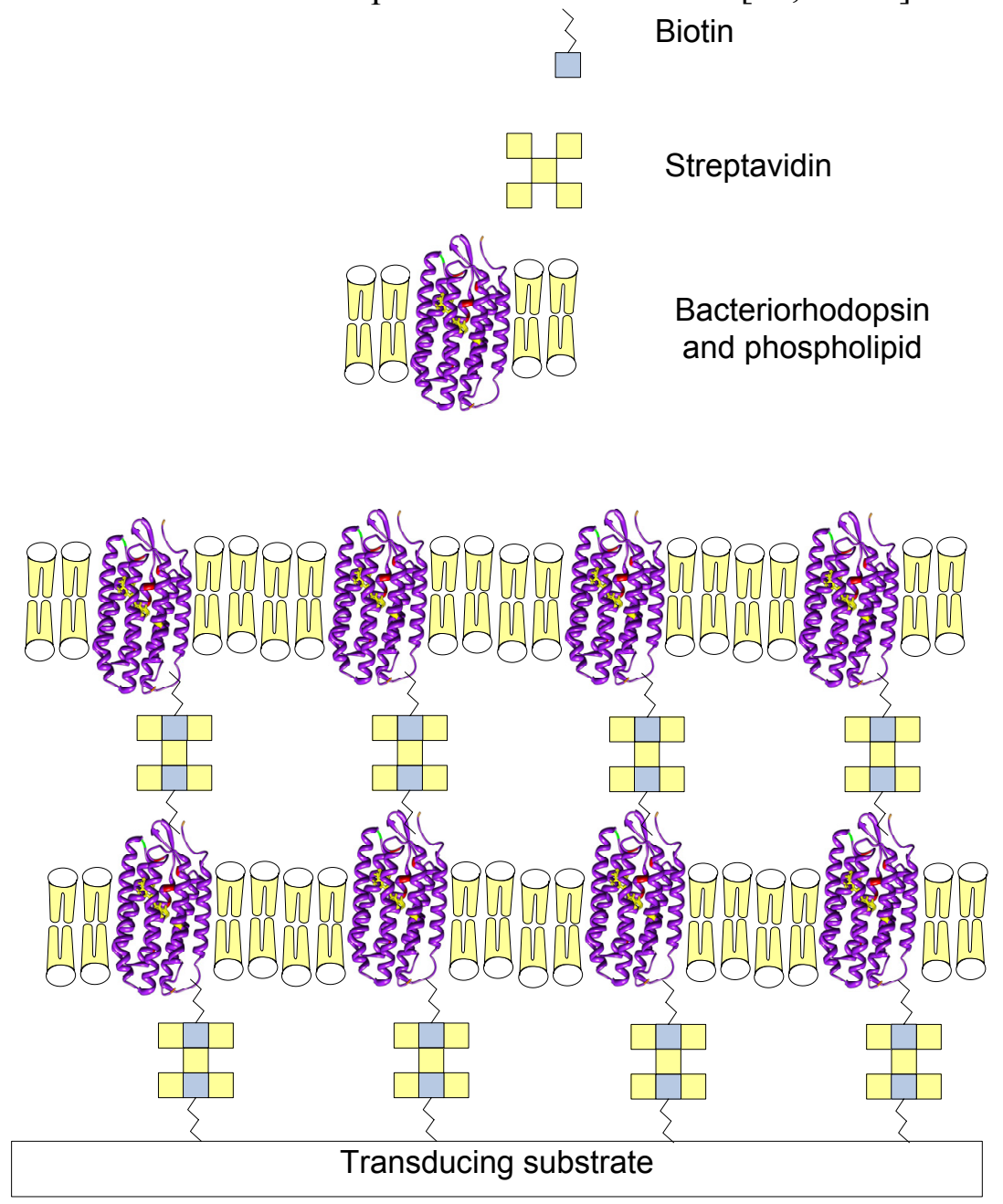

Figure 12. Possible layering scenario for Biotin and Streptavidin interaction.

\subsection{Patterning Methods}

For the purpose associated with this work "patterning" means a method to control the specific placement of material. In order to integrate bacteriorhodopsin with MEMS and 
NEMS devices, it must be selectively deposited on micro/nano pads. There are several patterning techniques that have been developed for patterning biological and inorganic macromolecules [51]. Photolithography has traditionally been a common method of patterning, but laser ablation and soft lithography techniques are also often used. Other patterning techniques include $\mu$-contact printing [52], dip-pen nanolithography [51], and $\mu$-fluidic patterning [53].

\subsubsection{Lithography}

Lithography can be envisioned as a stenciling process in which parts of an ultra-violet (UV) sensitive material are removed in a developer after exposure to a UV light through a mask. The light sensitive photoresist (PR) is a key component of lithography - when exposed to light the physical properties of the PR change. The type depends on whether a negative or positive photoresist is used. For a negative photoresist, the light exposed PR will remain after it is developed, whereas the light exposed PR is removed in the developer of a positive photoresist. Unless otherwise stated, the latter will be used in this project. Additionally, there are a variety of PR available, each with unique properties and capabilities (e.g., bonding capabilities with certain materials, reactivity with certain solvents, temperature thresholds).

In micromachining, lithography is a process in which two-dimensional images are transferred into three-dimensional structures through a layering process. In this process, the photoresist is usually a sacrificial layer. It is spun or sprayed onto a substrate to ensure it has a uniform thickness. The photoresist is then soft baked to increase the viscosity and stability. A two-dimensional image, or mask, is then placed over the layer of PR covering the substrate, and the entire unit is exposed to ultraviolet (UV) light. A three-dimensional image is created in the PR after the exposed PR is removed in the developer - the PR that was covered by the mask remains, the patterned photoresist is usually hard baked to solidify the PR. A secondary material can then be deposited on the photoresist. Generally, the photoresist and the secondary material are removed with a solvent (i.e. via lift off), leaving behind the patterned deposited material. This method could be utilized to pattern PM as demonstrated by [54].

\subsubsection{Patterning by Laser Ablation}

Haronian and Lewis [55] used laser ablation to pattern a broad film of electro-deposited $\mathrm{bR}$. This method of patterning began by electrophoretically depositing multilayers of $b R$ on a coated gold substrate. Next a $30 \mu \mathrm{m}$ thick electron microscope grid with evenly spaced $(30 \mu \mathrm{m})$ parallel bars was placed on top of the dried bR film. Then, the exposed bR film and the conducting layer under the bR film were removed with a Lambda Physik Model 103 argon fluoride excimer laser[51]. With the electron microscope grid mask removed, gold was sputtered across the surface of the bR strips and glass strips creating the second electrode (top electrode). The mask was then placed on the gold coated $b R$ 
and glass, but rotated $90^{\circ}$ from the original location. Again, the laser was used to ablate the exposed gold coated bR, leaving the bottom conductive layer $\left(\mathrm{SnO}_{2}\right)$ and creating an addressable pixilated surface of $(30 \mu \mathrm{m}$ x $30 \mu \mathrm{m})$ squares of bR but they did not verify electrical output of the patterned $b R$.

\subsubsection{Patterning by Plasma Etched Silicon}

Libertino et al. [56] demonstrated that it was possible to pattern PM on silicon ( $\mathrm{Si}$ ) by micromachining the sample surface and using silicon oxide $\left(\mathrm{SiO}_{2}\right)$ as the deposition surface. They accomplished this by first thermally growing a $25 \mathrm{~nm}$ layer of $\mathrm{SiO}_{2}$ on a $\mathrm{Si}$ wafer and then thermally growing a $200 \mathrm{~nm}$ thick layer of polycrystalline silicon on top of that. The polycrystalline silicon was masked and plasma etched down to the $\mathrm{SiO}_{2}$. The mask consisted of lines ( $600 \mathrm{~nm}$ wide and $2 \mathrm{~mm}$ long) and squares (600 $\mathrm{nm}$ per side) on the $\mathrm{SiO}_{2}$. The purple membrane was then deposited by soaking the patterned substrate in a PM solution (DDI water and PM) for 10 minutes and then drying under nitrogen leaving a PM in the trenches.

Patterning was accomplished by adsorbing the PM into the trenches and squares, however the patterned PM was not oriented or uniformly distributed and they did not measure a photoresponse for the deposited bR.

\subsubsection{Patterning by Soft Lithography}

Crittenden et al. [53] demonstrated patterning of PM by combining a metal coated polydimethylsiloxane (PDMS) $\mu$-fluidic channel network $(\mu \mathrm{FN})$ patterned template with electrophoretic deposition on a conductive substrate. The $\mu \mathrm{FN}$ pad was the inverse of a silicon master of $\mu$-fluidic channels. Then, to create the conductive $\mu \mathrm{FN}$ pad, $70 \mathrm{~nm}$ of Au was deposited by evaporation. The gold deposited on the $\mu \mathrm{FN}$ pad was then removed from the top surface of the pad with adhesive tape leaving conductive flow channels. The $\mu \mathrm{FN}$ pad and a counter electrode were cleaned. The counter electrode and the $\mu \mathrm{FN}$ pad were pressed together and a PM solution was then injected into the flow channels and a DC potential $(3-5 \mathrm{~V})$ was applied between the ITO and $\mu \mathrm{FN}$ pad for $5-30$ seconds. The patterned bR was then rinsed with deionized distilled (DDI) water and dried. With this method Crittenden et al. [53] were able to show with a scanning force microscope (SFM) that bR was deposited and patterned, but they were not able to measure a photoresponse, suggesting that either electrophoretic deposition or the process of patterning might have denatured the 10 monolayers of $b R$. 


\subsubsection{Pre-Patterned Substrate with LS Deposition}

Miyasaka et al. [57] describe another possible method for patterning bR. A pre-patterned two dimensional pixel array, as shown in Figure 13, was deposited on a silicon substrate. Then, monolayers of bR were deposited on the pre-patterned substrate using a LangmuirBlodgett trough modified to deposit LS monolayers of bR as discussed in [39]. The monolayers deposited were not highly oriented. Even though the monolayers deposited were not patterned directly, the substrate was and each pixel was addressable. This was accomplished by connecting a wire, via lithography, to each pixel. With this method, the photoresponse was measured using an electrochemical setup in which the top electrode was an electrolyte solution.

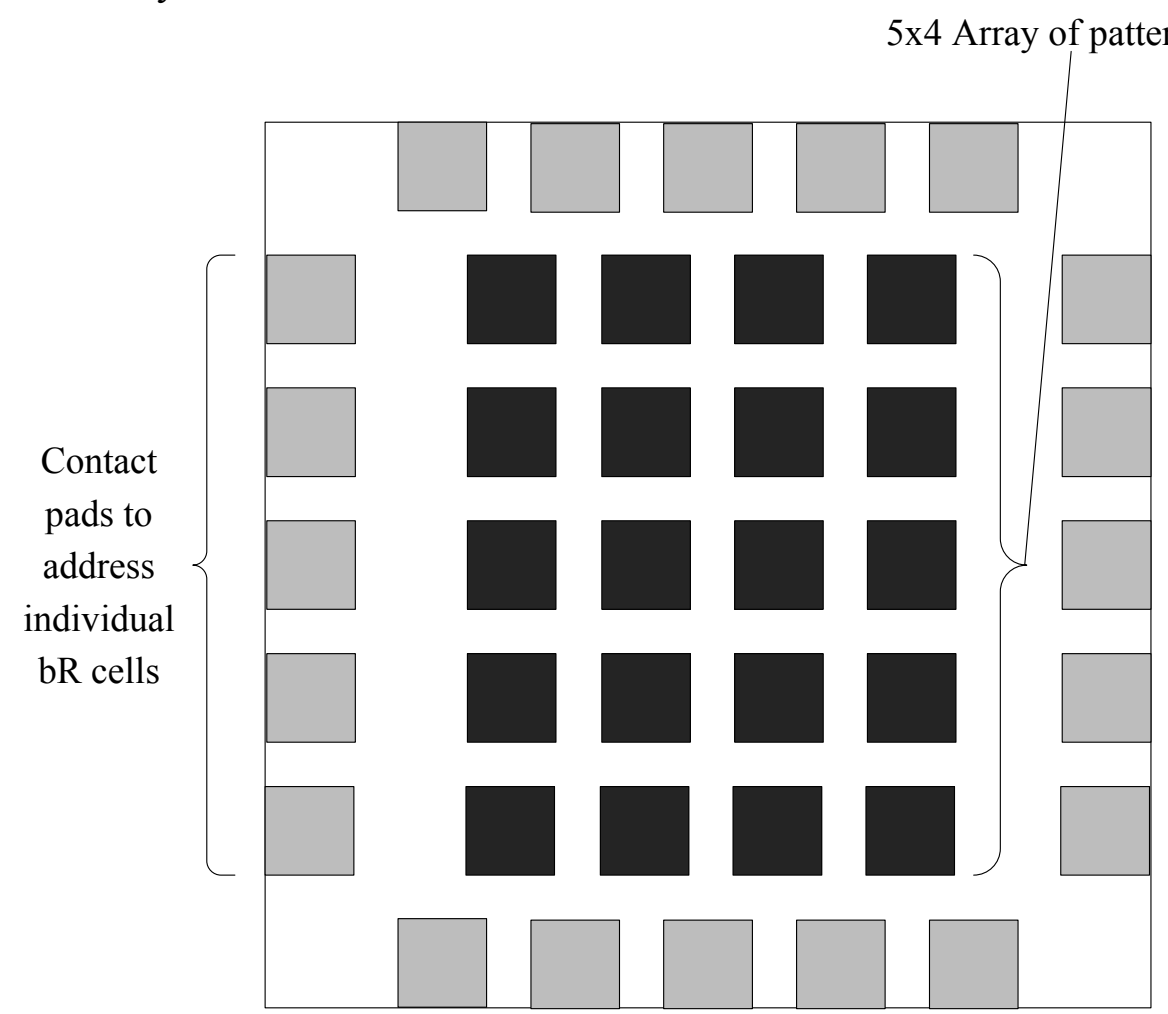

Figure 13. Schematic representation of the pre-patterned pixellated array of $b R$ photoreceptors from [57].

With this method there was control of the number of monolayers deposited, and the pattern but the orientation of the $\mathrm{bR}$ is questionable. 


\subsection{Single Electron Transistors}

A SET is a nano-scale device that exploits the quantum mechanical properties of electrons to switch on and off. Shown schematically in Figure 14, a SET fabricated by the Karre et al. [58] method have four components: gate, source, drain, and quantum islands but other have also developed SETs $[59,60]$. These components can be metallic or semiconductors. The SET function is based on the tunneling of electrons, quantized units of energy, from the source to the drain. For the tunneling to occur at room temperature the charge energy (Ec) must be greater then the thermal energy Ek as described in Eqs. (1) - (3).

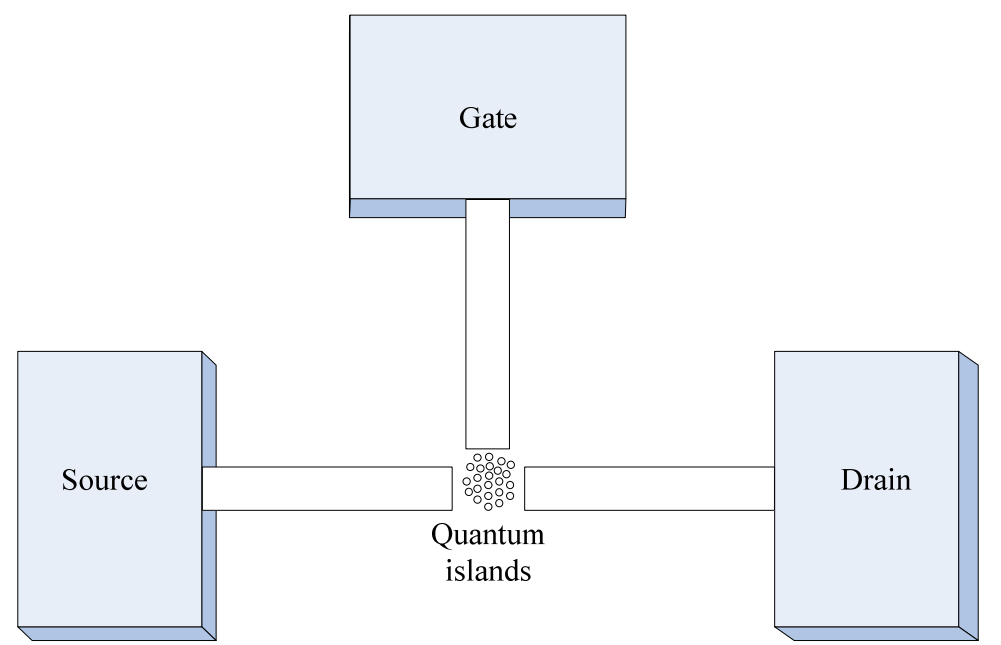

Figure 14. Schematic representation of a single electron transistor.

$$
\begin{gathered}
E_{c} \geq E_{K} \\
E_{K}=k T \\
E_{c}=\frac{e^{2}}{2 C^{*}}
\end{gathered}
$$

Where $\mathrm{E}_{\mathrm{k}}$ is equal to $25.875 \mathrm{meV}$ at room temperature, $\mathrm{T}=300 \mathrm{~K}$ and $\mathrm{k}=1.38 \times 10^{-23} \mathrm{~J} / \mathrm{K}$, $e$ is the charge of an electron $\left(1.6 \times 10^{-19} \mathrm{C}\right)$, and $\mathrm{C}^{*}$ is the total device capacitance. Examining the equations above one can see that there are variables $\mathrm{T}$ and $\mathrm{C}^{*}$ which can be used to control the functionality for multi QDs arrays and single QD SET devices. One way is to reduce the thermal energy. The thermal energy can be reduced by decreasing the operating temperature. The other way is to reduce the total device capacitance $C^{*}$, which would increase the charging energy, hence increasing its operating temperature. This can be accomplished by reducing the size of the QDs (QDs $<10 \mathrm{~nm}$ in diameter) and deceasing the QD separation to $<2 \mathrm{~nm}$-in the gap separating the source, drain, and gate. 
A single electron device is based on the tunneling of electrons from the source to the drain. Controlling the tunneling of the electrons between the source and drain describes a SET. The controlled tunneling is accomplished by the addition of the gate electrode. The gate electrode can be located on the side, top, bottom, or combination of these architectures around the source and drain electrodes. Generally speaking, increasing the gate voltage causes the attraction of electrons to the metallic QDs, increasing the tunneling effect. Shown in Figure 15 is a phenomenon known as Coulomb blockade which occurs when there is no tunneling of the electrons between the source, islands, and drain to create a current. Coulomb blockade can be removed by adding a gate voltage to align energy levels on the quantum islands with that of the source and drain [61, 62]. This allows electrons to tunnel between the source and drain. Modulating the gate voltage can produce the on-off functionality of the SET.

For a multi and single QD single electron device to be considered a SET, the gate voltage versus source-drain current, as shown in Figure 15, must fluctuate with the gate voltage. For a single dot QD SET the $\mathrm{V}_{\mathrm{DS}}$ versus $\mathrm{V}_{\mathrm{G}}$ fluctuation are regularly shaped and spaced as shown by $[60,63-65]$. The peaks and valleys represent the addition and subtraction of a single electron from the QDs by modulating the gate voltage. Figure 15 also shows the $\mathrm{I}-\mathrm{V}$ and differential conductance characteristics of a SET, indicating that within the coulomb blockade region the conductance is zero, which means that no current is passing between the source and drain. 

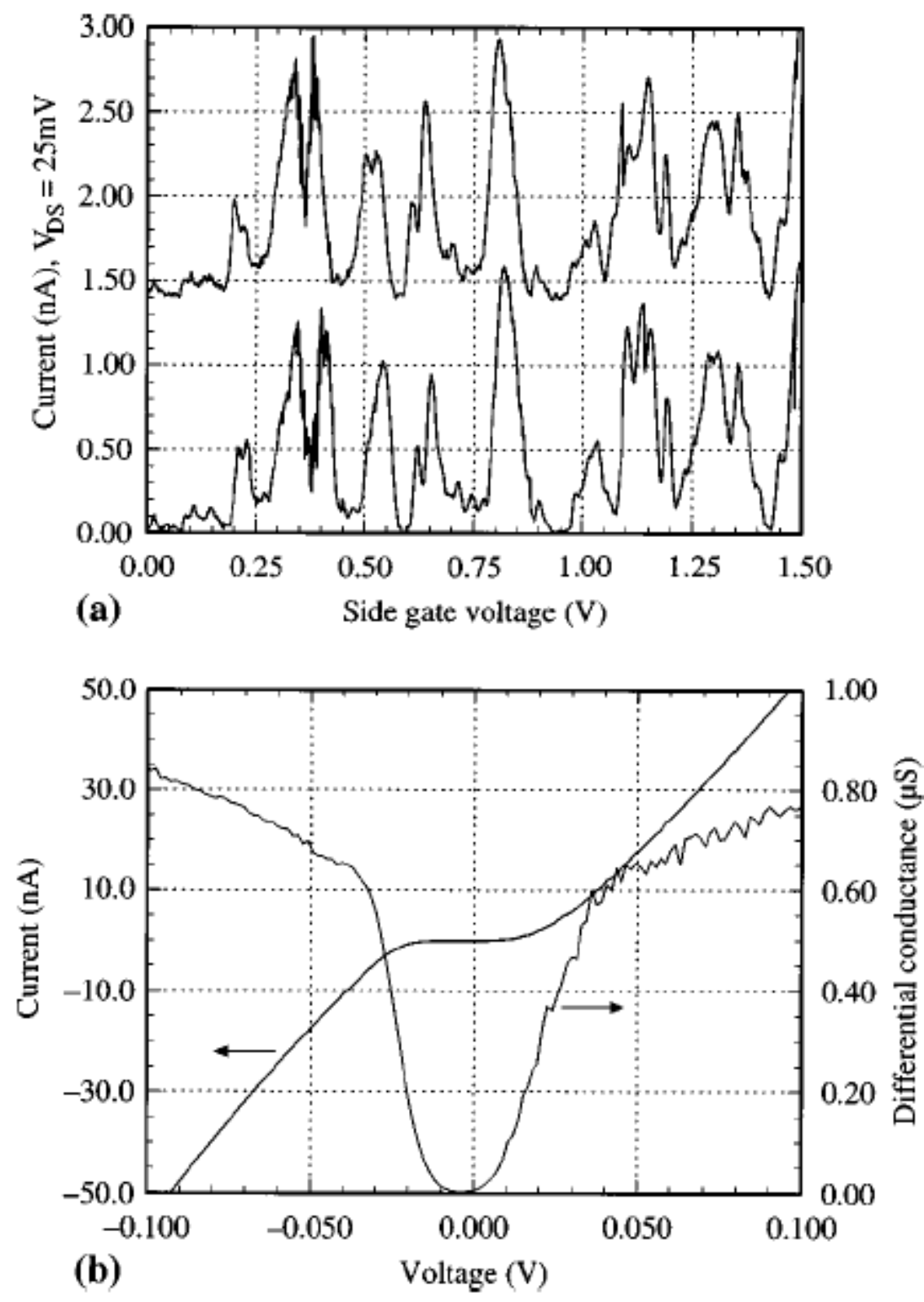

Figure 15. Gate conductance oscillations at a source-drain bias of $25 \mathrm{mV}$. The upper curve is the reverse sweep of the lower curve and is offset by $1.4 \mathrm{nA}$. Both side gates are biased together. (b) The current-voltage characteristic and differential conductance at 4.2 K. [64]

Reproduced by permission of the publisher of Smith, R.A. and H. Ahmed, A silicon Coulomb blockade device with voltage gain. Applied Physics Letters, 1997. 71(26): p. 3838-3840 Figure 2. 


\subsection{Background Conclusions}

A variety of methods that have been used to orient, deposit, and pattern purple membrane have now been described. There have been numerous attempts to deposit monolayers of purple membrane, but fewer attempts to deposit oriented monolayers. Even those methods, which say they incorporate orientation of monolayers, need to be further investigated to determine the degree of orientation. Electrophoretic deposition has been used to orient, pattern, and deposit 10 to 1000's of monolayers of bR but with limited control of the number of monolayers deposited and the degree of orientation depends on $\mathrm{pH}$ of the solution. The only known method to achieve a highly oriented monolayer has been with the chemical interactions (i.e., thiols and gold, and biotin and streptavidin) but a multilayer system structure was not achievable with this method.

From this literature review, methods of immobilizing bR on a solid electrode were reviewed and these methods include electrophoretic deposition, Langmuir-Blodgett deposition, Langmuir-Schaffer deposition, electrochemical layering/self assembled monolayers/ionic self-assembly, and chemical binding of biotin and streptavidin. Since most of these studies report different methods of quantifying the degree of orientation of the deposited bR this suggests that highly oriented uniform multiple monolayers may not be achievable because of the all the factors that affect it.

The literature review also shows that the majority of studies of bR have been conducted in the aqueous state, few studies have been conducted on bR in the dried state.

The methods of patterning bR that were reviewed include laser ablation into pixels, absorption into micro-machined trenches in silicon, $\mu$-contact printing, and LangmuirSchaffer deposited on a pre-patterned substrate. The only method with measurable photoresponse was the last method using an electrochemical cell. For all other methods there was no measurable photoresponse following the processing.

SETs have tremendous potential in practical applications due to their nanosize, ultra-low power requirements, and electrometer-like sensitivity. In this research, the bR will be applied to the gate of the SET and act as the voltage source which will modulate the electrons the flowing between the source and drain. When the bR is light activated it will act as a gate voltage changing the tunneling barrier between the source and drain, which may be used to control the tunneling of electrons between the source and drain as shown in Figure 15. The SETs for this research were fabricated by a process described by Karre et al. [58]. 


\section{Chapter 3 \\ Purification and Verification of Purple Membrane}

\subsection{Purification}

Crude PM preparations were obtained from Halobacterium salinarum that had been produced in the presence of white light exposed under anaerobic conditions at $40{ }^{\circ} \mathrm{C}$ for 5 -7 days. The bacterial cells were then pelleted and osmotically lysed with water and DNase at a concentration of $1 \mathrm{unit} / \mathrm{mL}$. After lysis, the crude preparation was centrifuged at $3000 \mathrm{~g}$ for $15 \mathrm{~min}$ to remove cellular debris, the pellet was discarded and the supernatant was kept. The remaining solution was washed using a process called differential centrifugation, where the solution was centrifuged at $151,000 \mathrm{~g}$ for $30 \mathrm{~min}$, supernatant was discarded, and the pellet was resuspendend by brief sonication in fresh DDI and again centrifuged and repeated till the supernatant was clear following centrifugation [15]. The final purification of PM as shown in Figure 16, was obtained by subjecting the crude PM to equilibrium density gradient centrifugation (174,000 $\mathrm{g}$ for 20 hrs at $4{ }^{\circ} \mathrm{C}$ ) through preformed linear sucrose gradients [66]. The bR was extracted from the sucrose gradient by hand using a glass pipette.

\subsection{Verification}

An analysis of the purified bR was performed, as shown Figure 17 and Figure 18, using sodium dodecyl sulfate-polyacrylamide gel electrophoresis (SDS-PAGE). This verified the purity of the $b R$ and the molecular weight of the bR protein. SDS is an anionic detergent that promotes the unfolding and denaturation of the protein to form a rod shaped structure. The proteins have a constant charge to mass ratio and their MW can be resolved as a function of size in the SDS-PAGE. 

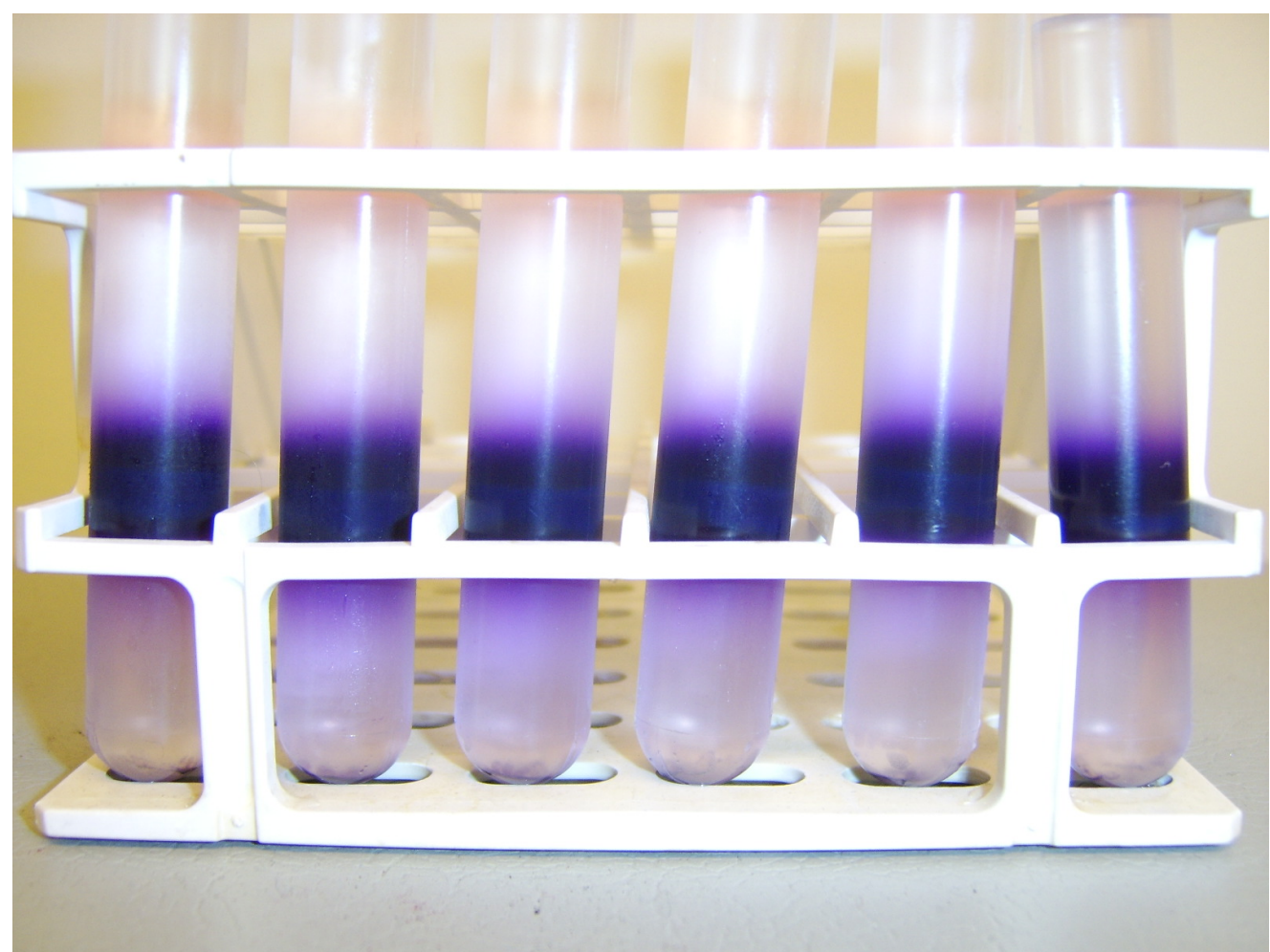

Figure 16. Final purification step, the bR was fractioned in the linear sucrose gradient.

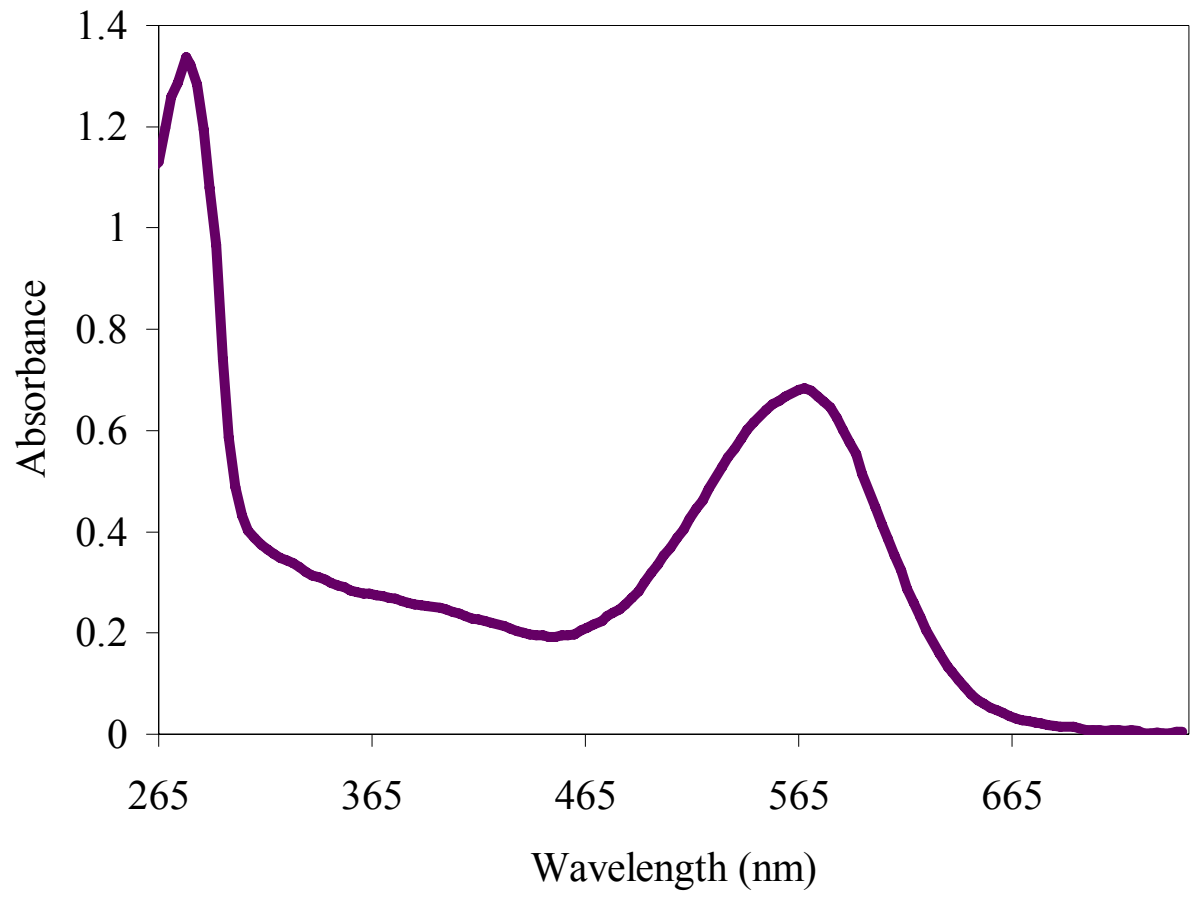

Figure 17. Absorbance spectrum of $b R$. 


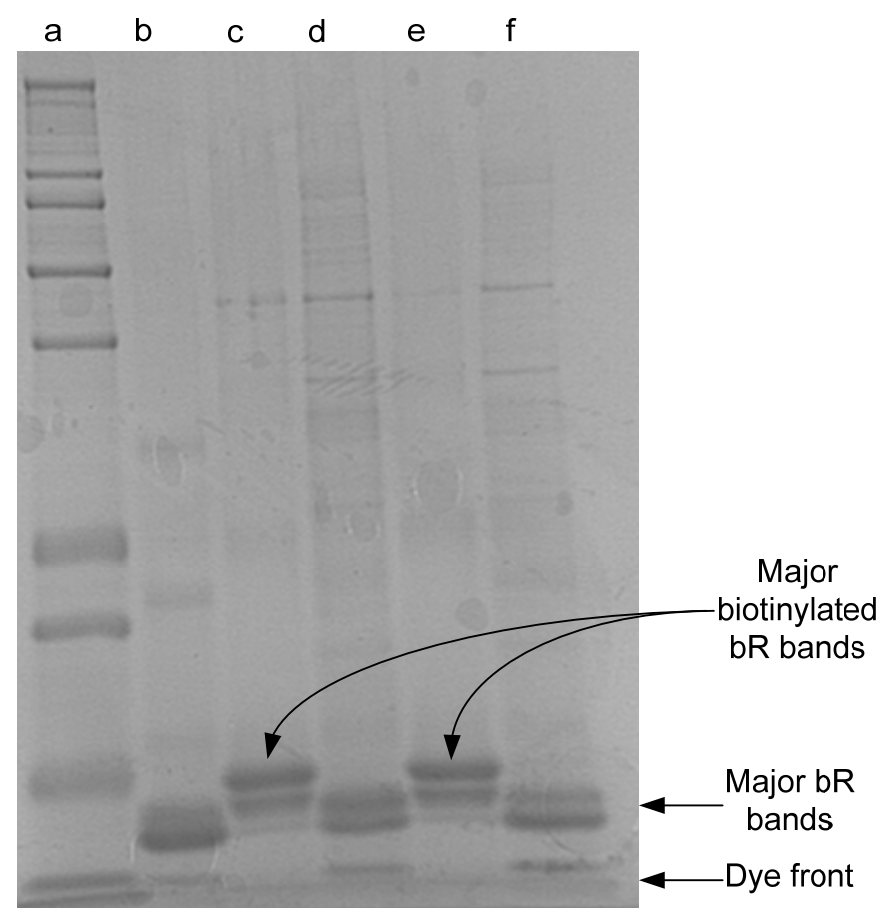

Figure 18. SDS gel, Lane a - Mark12 standard, Lane $\mathrm{b}-\sim 2 \mu \mathrm{g}$ purified bR post-sucrose gradient, Lane $\mathrm{c}$ and $\mathrm{e}-\sim 2 \mu \mathrm{g}$ biotinylated $\mathrm{bR}$, Lane $\mathrm{d}$ and $\mathrm{f}-\sim 2 \mu \mathrm{g}$ crude bR prep presucrose gradient

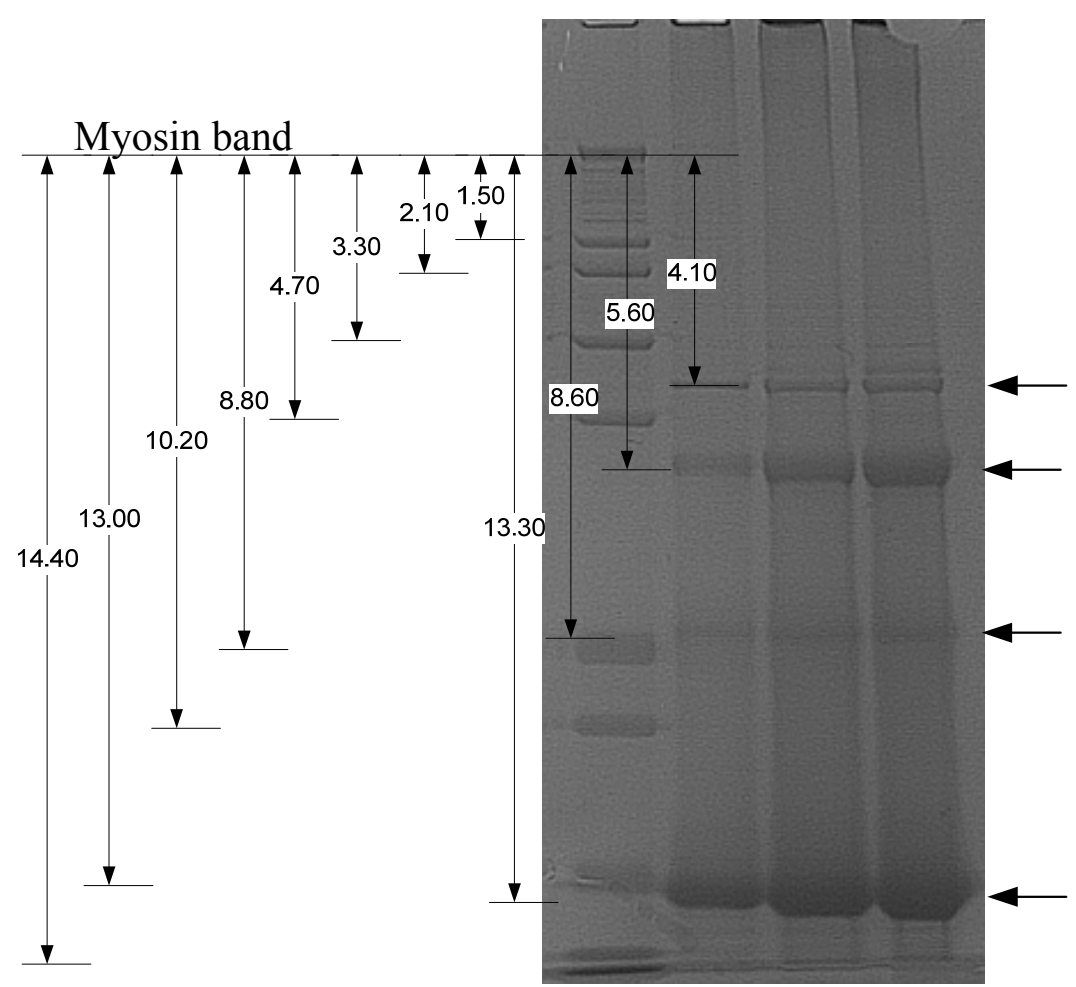

Figure 19. SDS gel plotted with migration distances from myosin, shown for both the mark12 standard (left column) and bacteriorhodopsin remaining columns. 
The SDS-PAGE has a number of vertical lanes. Each lane corresponds to a sample well. During electrophoresis, the samples migrate a distance from the application well into the gel depending on their relative size and charge. In Figure 18 and Figure 19, a molecular weight (MW) standard was in the left lane. If performed properly, SDS is a good process to determine purity as shown in Figure 18. In contrast, the samples in Figure 19 were overloaded, as indicated by the bulging bands, thus this gel was not a good indicator of purity but still indicates the relative positions of bR in the SDS Gel.

All samples prepared for the SDS-PAGE had a total volume of $10 \mu \mathrm{L}$ and consisted of $3.5 \mu \mathrm{L}$ of NuPAGE LDS tracking dye, $0.5 \mu \mathrm{L}$ of Mercaptoethanol as the reducing agent, $2.0 \mu \mathrm{g}$ protein by volume, and DDI water to final volume. The samples were heated to $70-80{ }^{\circ} \mathrm{C}$ for 10 minutes and then pipetted into the wells of the polyacrylamide gel. Gels were run for $\sim 10$ minutes at 100 volts and an additional $\sim 40$ minutes at 200 volts at $\sim 20{ }^{\circ} \mathrm{C}$ in a MOPS buffer solution. The gels were then removed, stained in SimplyBlue Safe Stain, digitally imaged, and analyzed. From the standardized SDS-PAGE procedure with a $10 \%$ bis tris gel, it appeared that the purified bR displayed a MW of $\sim 20 \mathrm{kDa}$, an unexpected value that does not agree with what others have reported using SDS-PAGE [15, 66-69] which reported that $26 \mathrm{kDa}$ is correct. After further investigation described in the next paragraph, it was determined that $20 \mathrm{kDa}$ for bR measured using SDS-PAGE.

A detailed MW analysis of the SDS-PAGE gel shown in Figure 19 and Figure 20 indicate that the Mark12 standard follows a relatively straight line on a log-log scale, as expected, and the major bR band (dark band) has a MW of $\sim 20 \mathrm{kDa}$. The $20 \mathrm{kDa}$ was interpolated based on its location with respect to the known bands of the Mark12 standard. The journal articles that reported a MW of $\sim 26 \mathrm{kDa}$ with SDS did not report the use of standardized procedures. In contrast, Bridgen and Walker [70] reported a SDS procedure and that $\mathrm{bR}$ had an apparent molecular weight of $20 \mathrm{kDa}$ in the presence of SDS which was significantly lower than values obtained by other techniques. With this study we concluded that $\mathrm{bR}$ was being produced based on the MW agreement with Bridgen and Walker [70] and the bR was pure as indicated by the SDS-Gel and the absorbance ratio $\left(\mathrm{A}_{280} / \mathrm{A}_{570}\right)$ of $\sim 2$ [15] as shown in Figure 17, indicating that our isolation procedure yielded high purity $b R$.

To further analyze the purple membrane being produced and purified, a preformed linear sucrose gradient and ultracentrifugation $\left(174,000 \mathrm{~g}\right.$ for $20 \mathrm{hrs}$ at $4{ }^{\circ} \mathrm{C}$ ) were used to determine the intrinsic density/buoyant density. As shown in Figure 21 a purple membrane band appeared where the density of the sucrose gradient equals the buoyant density of the PM, which occurs at $\sim 1.17 \mathrm{~g} \mathrm{~mL}^{-1}$. 


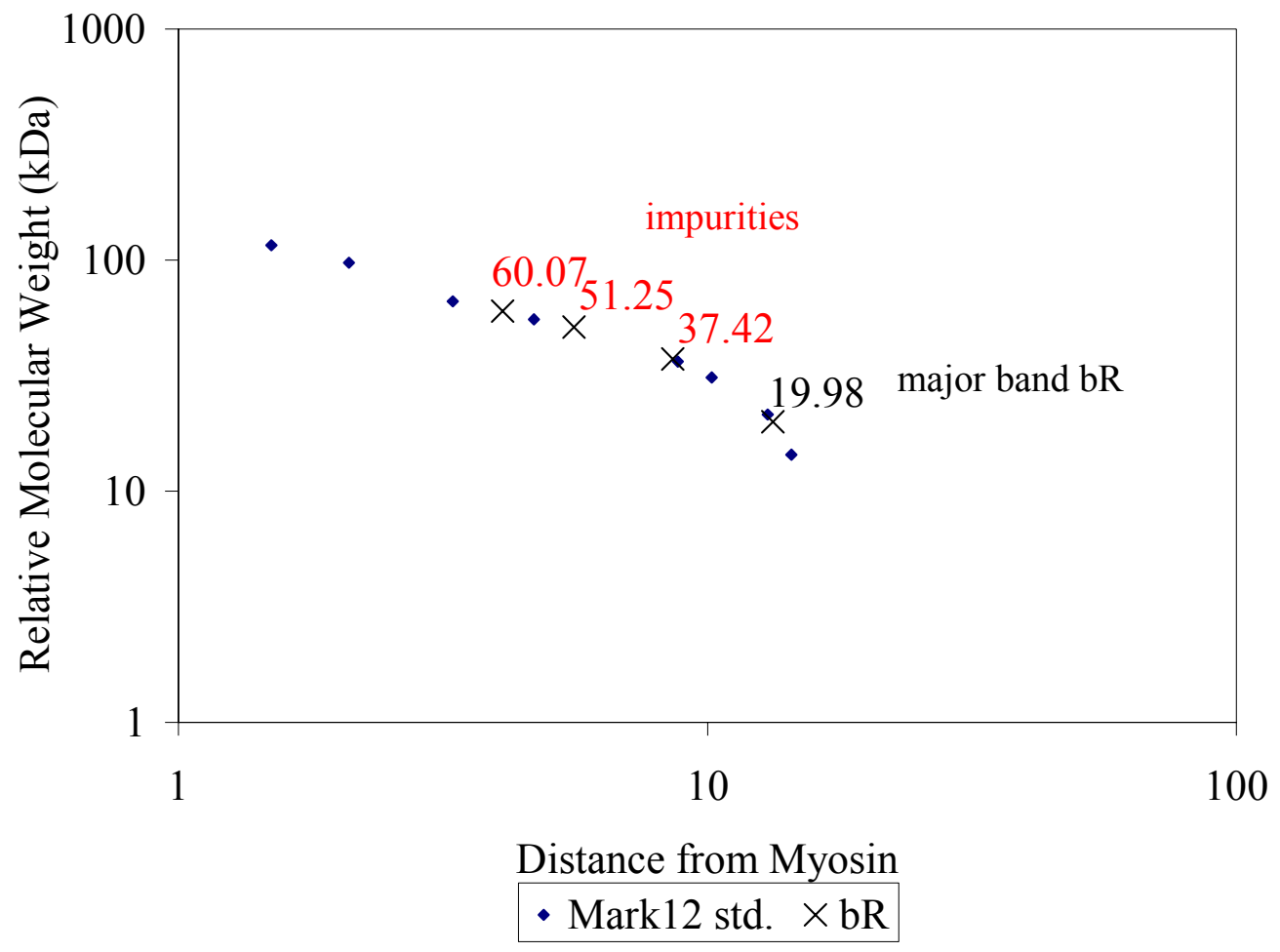

Figure 20. Log-log plot of the relative molecular weight vs. migration distance from Myosin, Mark12 standard and bR.

Figure 21. Post-centrifugation of PM in linear sucrose gradients. The PM band formed at a density of $\sim 1.17 \mathrm{~g} \mathrm{~mL}^{-1}$. 
Two studies were performed, one using a sucrose gradient from $35-42 \mathrm{w} / \mathrm{w} \%$ yielding a density in the range of $1.15-1.20 \mathrm{~g} \mathrm{~mL}^{-1}$ and a refined sucrose gradient from $36-41 \%$ yielding a density in the range of $1.155-1.18 \mathrm{~g} \mathrm{~mL}^{-1}$. To determine the buoyant density of PM, the linear sucrose gradients were hand fractionated into $0.2 \mathrm{~mL}$ aliquots, the refractive index (RI) and absorbance for each aliquot was measured. The measured RI was converted to density using a standard conversion table for sucrose at $20{ }^{\circ} \mathrm{C}$, and interpolation. Results of these tests are shown in Figure 22 and Figure 23, respectively.

The data and linear regression analysis indicates a linear trend, indicating that a quality linear gradient was achieved. As expected, the sucrose gradient with a larger range has a steeper slope, shown in Figure 22 compared to Figure 23, but both have a linear correlation of approximately 0.98 . Since the slope of the sucrose was smaller in Figure 23 , the peaks at $280 \mathrm{~nm}$ and $570 \mathrm{~nm}$ were further resolved.

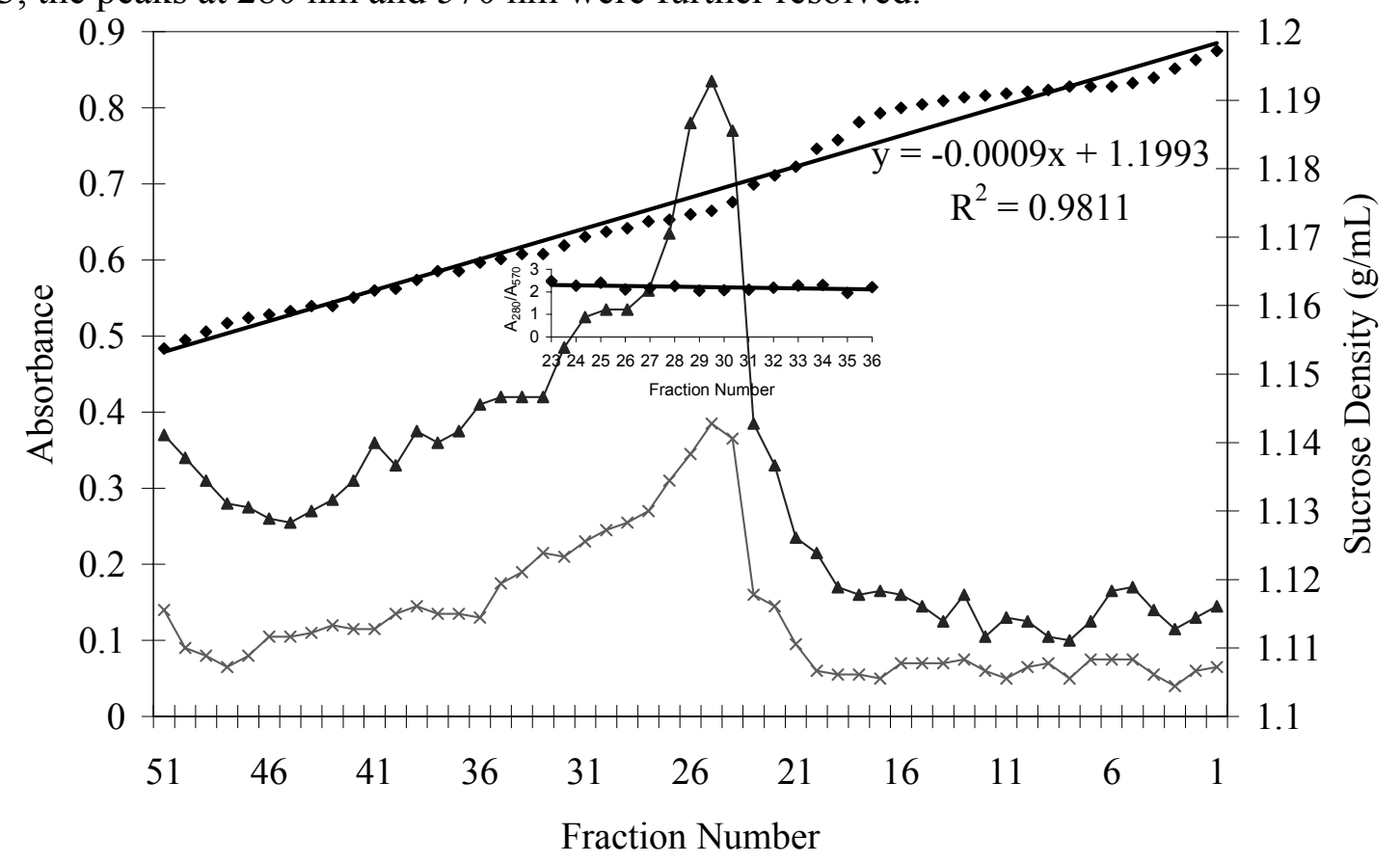

- Surcose Gradient $\rightarrow 280 \mathrm{~nm} * 570 \mathrm{~nm}$ - Linear (Surcose Gradient)

Figure 22. Sucrose density and absorbance of $\mathrm{PM}$ as a function of fraction number for linear sucrose gradient from $35 \%$ to $42 \%$ by weight, inset shows $\mathrm{A}_{280} / \mathrm{A}_{570} \sim 2$. 


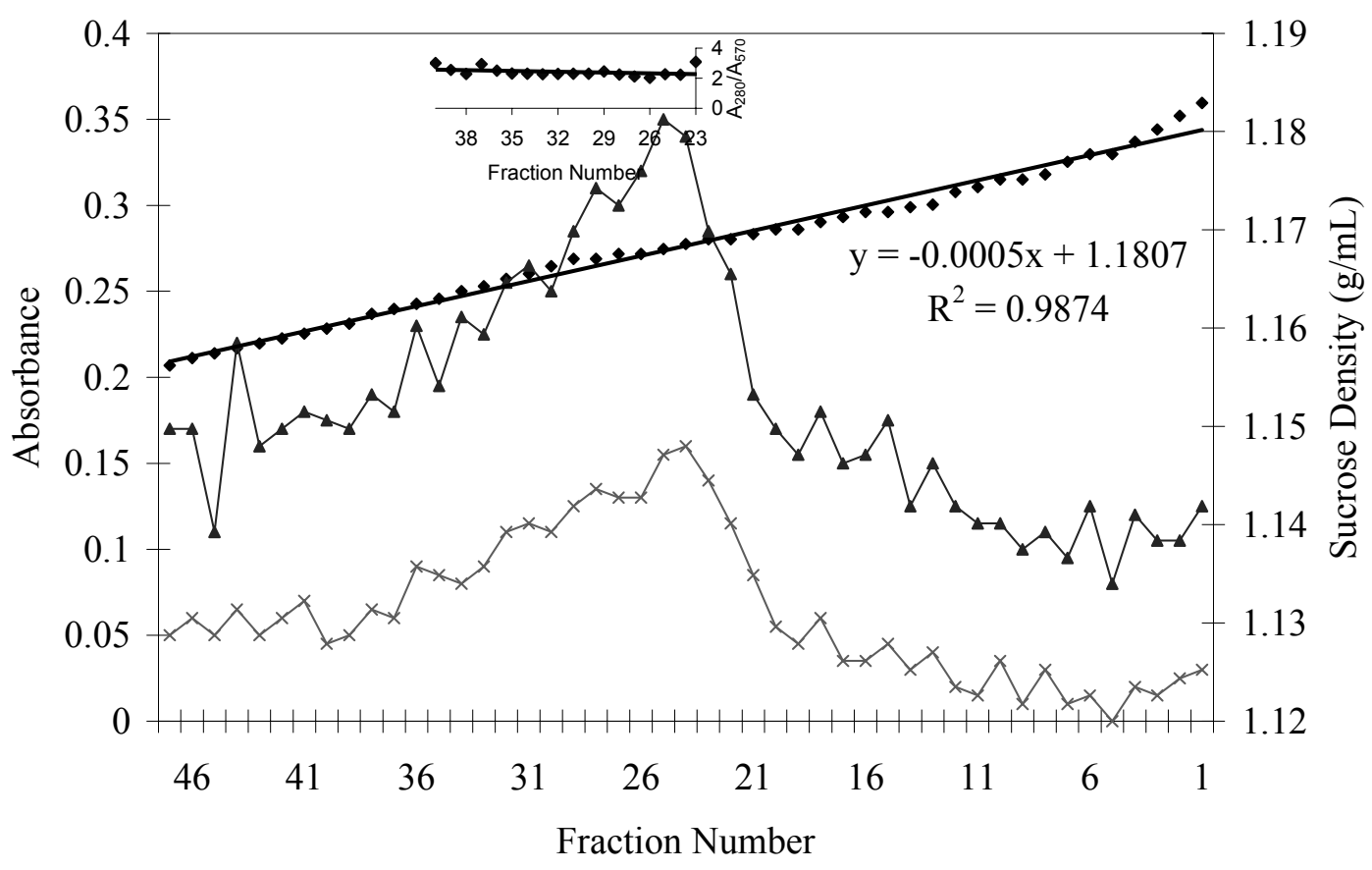

- Surcose Gradient $\leftarrow 280 * 570-$ Linear (Surcose Gradient)

Figure 23. Sucrose density and absorbance of $\mathrm{PM}$ as a function of fraction number for linear sucrose gradient from $36 \%$ to $41 \%$ by weight, inset shows $A_{280} / A_{570} \sim 2$.

In Figure 22 and Figure 23 the violet and green curves display the absorbance $280 \mathrm{~nm}$ and $570 \mathrm{~nm}$, respectively, for each fraction. In Figure 22 and Figure 23 the peak absorbance occurs at the location with the densest amount of PM in the linear sucrose gradient fraction \# 25 and \# 23, respectively. It appears that there were two minor shoulders of dense PM. These two minor shoulders have a density that was less than the peak density, indicating that the percentage of the lipid and protein was different than the $75 \%$ protein and $25 \%$ lipid as reported in previous papers such as [68]. As expected, the resolved linear sucrose gradient has a less dramatic peak and broader shoulders (Figure 23). The PM equilibrium density from was $\sim 1.1738 \mathrm{~g} \mathrm{~mL}^{-1}$ and $\sim 1.1685 \mathrm{~g} \mathrm{~mL}^{-1}$, respectively. From this experimental data we estimated the intrinsic density, to be $\sim 1.17$ $\mathrm{g} \mathrm{mL}^{-1}$. This density value was in close agreement with the $\sim 1.18 \mathrm{~g} \mathrm{~mL}^{-1}$ obtained by other researchers [66-68].

The graph in the middle of Figure 22 and Figure 23, $\mathrm{A}_{280} / \mathrm{A}_{570}$ versus fraction number, indicates the $\mathrm{bR}$ had not been chemically modified, since the $\mathrm{A}_{280} / \mathrm{A}_{570}$ was relatively constant and $\sim 2$. This relationship of the retinal and protein complex can be quantified based on the absorbance of the amino acids with aromatic rings phenylalanine, tyrosine, and tryptophan for the ultraviolet peak at $280 \mathrm{~nm}$ and the retinal for the visible peak at $570 \mathrm{~nm}$. The ratio of these two peaks should remain constant $\sim 2$ and should not change with respect to concentration or from batch to batch. A dramatic shift, $+/-1$, in the $\mathrm{A}_{280} / \mathrm{A}_{570}$ could indicate either retinal disassociation and/or protein denaturation. 
From the intrinsic density of PM, one can theoretically calculate the composition, the percentage of lipid and protein content. The percentage of lipid and protein was estimated using different methods. The first method was based on the protein and phospholipid density given by Tristram-Nagle et al. [68]. The protein density was 1.259 $\mathrm{g} \mathrm{mL}^{-1}$ and the phospholipid density ranged from $1.01-1.064 \mathrm{~g} \mathrm{~mL}^{-1}$. Using a phospholipid density of $1.06 \mathrm{~g} \mathrm{~mL}^{-1}$ the percent composition of $\mathrm{PM}$ was $55 \%$ protein and $45 \%$ lipid, which is quite different from what others have reported [66-69].

However, if the same value of the protein density was used, $1.259 \mathrm{~g} \mathrm{~mL}^{-1}$, with the phospholipid density of $0.89 \mathrm{~g} \mathrm{~mL}^{-1}$ from [67], then the approximate percent of protein and lipid in PM was $76 \%$ to $24 \%$, respectively, with a PM density of $1.17 \mathrm{~g} \mathrm{~mL}^{-1}$ which is nearly the same that has been reported in the literature [66-68]. A consensus has not been reached regarding the density of the protein and the lipid in PM. Therefore it was difficult to determine which set of calculations was most accurate. However, a change of protein-to-lipid concentration was still determined so long as a consistent methodology was employed for all calculations. This change in protein lipid concentration can be seen in both Figure 22 and Figure 23. To the left of the absorbance peak, the PM has a higher density and to the right a lower density. This density discrepancy could be caused by a difference in the protein to lipid concentrations. So, for larger density $\left(>1.17 \mathrm{~g} \mathrm{~mL}^{-1}\right)$ one would expect a greater concentration of protein and for a smaller density $\left(<1.17 \mathrm{~g} \mathrm{~mL}^{-1}\right)$ one would expect a greater concentration of lipid. This change in the PM protein and lipid concentration was slightly more pronounced when the PM preparation was sonicated.

\subsection{Purification and Verification of Purple Membrane Conclusions}

Through the use of SDS-PAGE, it was shown that our bR does not migrate to the prescribed $26 \mathrm{kDa}$ in the presence of SDS but rather $\sim 20 \mathrm{kDa}$. Using linear sucrose gradients and ultracentrifugation it was determined that our bR has a buoyant density around $1.17 \mathrm{gm} \mathrm{mL}^{-1}$. From these experiments it was determined that the protein produced and purified was indeed bacteriorhodopsin. 


\section{Chapter 4 \\ Purple Membrane Minimization and Monomerization}

As mentioned earlier, bR is found in PM patches which have a diameter of about $\sim 500$ $\mathrm{nm}$. The purpose of these studies was to determine if sonication or detergent decreases the PM patch size from $500 \mathrm{~nm}$ in diameter to 10's of $\mathrm{nm}$ in diameter while maintaining functionality of the $b R$. The size of the material controls the minimum patterning size, packing density of $\mathrm{bR}$, and possibly the functional characteristics related to activating SETs. One way to decrease the minimum patterning size would be to decrease the size of the PM patches, or better yet form monomers of $b R$. Two possible methods for reducing the PM patches were investigated. The first method was by sonication, a physical method of breaking up the PM patches with sonic pulses. The second method was dissolving the PM patches in an Octyl $\beta$-D-glucopyranoside (OG) detergent to form monomers of $b R$. The second method proved to be more successful by using a combination of ultracentrifugation and gel filtration chromatography and is described in more detail in the following section. Successful extraction and handling of the monomerizied bR would broaden its applicability in hybrid micro- and nano- devices.

\subsection{Sonication of PM}

In this section, the effects of sonication of PM are discussed. From this research, it was determined that sonication of PM had limited success in decreasing the patch size based on size exclusion chromatography. The only noticeable difference, based on the ultracentrifugation of linear sucrose gradients, occurs after 2 minutes of sonication. Additional sonication had no significant effect on decreasing the patch size.

Disruption by sonication of tightly packed bR molecules that comprise the PM was examined. The effect of sonication was examined by re-suspending PM at a concentration between $1-2 \mathrm{mg}$ in $1 \mathrm{~mL}$ of DDI water and then subjecting the membrane to sonication. A Branson 450 sonicator equipped with a microprobe was operated in the range of $30-55 \%$ power amplitude, 0.2/2 seconds ON/OFF pulse regimen. Total sonication time ranged from $2-24$ minutes. During sonication, the sample temperature was monitored with an Omega HH509 thermocouple and controlled with an ice bath. The sample temperature never exceeded $25^{\circ} \mathrm{C}$.

\subsubsection{Ultracentrifugation of Linear Sucrose Gradient}

The initial sonication experiment was conducted for 2 minutes. The sonicated PM was then evaluated by equilibrium density gradient centrifugation as shown in Figure 24 and 
Figure 25, identical to the methods employed for purifying and determining the intrinsic density of PM as previously shown. The dispersed PM shown in Figure 24 indicated that sonication had modified the intrinsic density of the PM, suggesting that the PM patch size had been decreased.

Figure 24. Sonicated PM in preformed linear sucrose gradient following ultracentrifugation.

Analysis of the gradient fractions revealed that $92 \%$ of the sonicated PM was recovered in the density range between 1.13 to $1.16 \mathrm{~g} \mathrm{~cm}^{-3}$, from Figure 25. This PM material also retained its characteristic $\mathrm{A}_{280} / \mathrm{A}_{570}$ of $\sim 2$, indicating that the sonicated PM had not been chemically modified. This result differed sharply from those obtained with the untreated PM where $75 \%$ of the recovered material was localized in the density range from 1.16 to $1.175 \mathrm{~g} \mathrm{~cm}^{-3}$, as shown in Figure 22. Since the observed change in the average PM buoyant density cannot be attributed solely to alterations in the PM intrinsic density, these results suggest that the average size of the sonicated PM had decreased. 


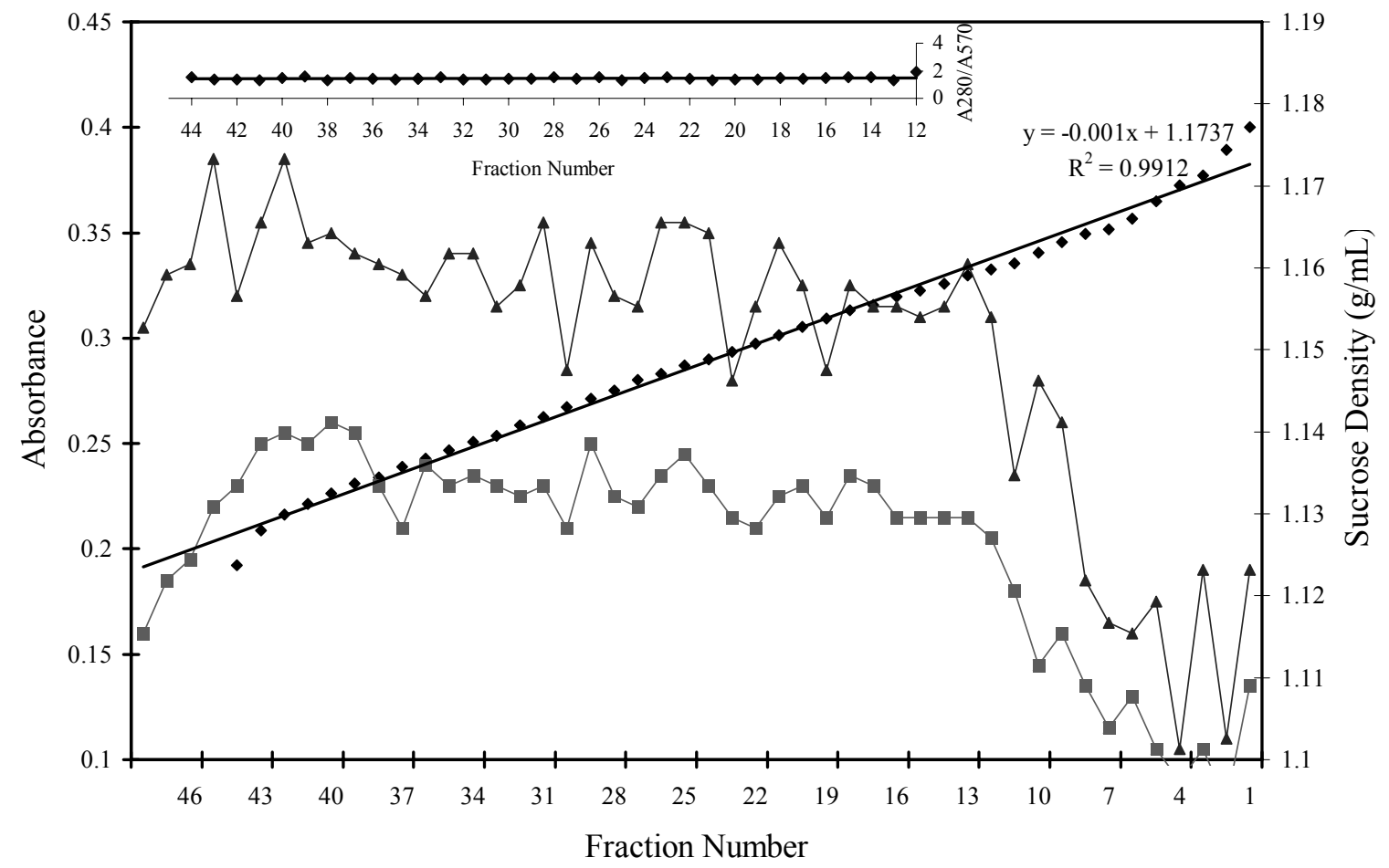

- Sucrose Gradient $\longrightarrow 280 \mathrm{~nm}-570 \mathrm{~nm}$

Figure 25. Sonicated purple membrane distribution following equilibrium density gradient ultracentrifugation.

\subsubsection{Gel Size Exclusion Chromatography}

Gel size exclusion chromatography was used to determine if sonication decreased the PM patch size. The sonicated material was separated on a Sepharose $2 \mathrm{~b}$ (molecular weight exclusion limit $25 \times 10^{6} \mathrm{Da}$ ) column, $14 \mathrm{~cm} \mathrm{x} 1 \mathrm{~cm}$, equilibrated with $0.45 \mu \mathrm{m}$ filtered DDI water. Gel size exclusion chromatography is a method used to determine the size of molecules in solution. The globular size of molecules is related to the time or the number of void volumes that pass through the column before the molecule elutes. Therefore, larger molecular weight molecules would be eluted from the column first while smaller molecular weight molecules require a longer time period and larger volume of eluting buffer. PM samples at concentrations of $1.11-3.50 \mathrm{mg} \mathrm{mL}^{-1}$ were suspended in $200-$ $500 \mu \mathrm{L}$ of filtered DDI water, and applied to the column. Aliquots of $200 \mu \mathrm{L}$ were collected using a Gilson model FC-80k fractionator. Column void volumes of $1.8-2.0$ $\mathrm{mL}$ were measured from a non-sonicated PM sample preparation. Analysis of the column fractions following 2 minutes of sonication, shown in Figure 26, did not indicate any significant change in the physical state of PM between sonicated and non-sonicated samples. 
The sonication power was increased to $45 \%$ with the same pulse regimen and the total sonication time was increased to 6 minutes. These results are shown in Figure 27. The absorbance measurements before and after the experiment indicated that $\sim 99 \%$ of the non-sonicated samples were recovered. The decrease in the peak absorbance of the sonicated vs. the non-sonicated samples shown in Figures 30 and 31, indicated that < $100 \%$ of the sonicated sample was recovered. This was further confirmed by visual inspection of the column which had a slight purple tinge after the sonicated sample was eluted. The loss of purple membrane was caused by the retention of the PM in the Sepharose $2 \mathrm{~b}$ column and aerosol loss during sonication.

The data indicated that sonication had limited success decreasing the patch size since the majority of the sonicated PM eluted through the column in the same number of voids as the non-sonicated. But as shown in Figure 26, with 2 minutes of sonication, $\sim 14 \%$ of the PM was retained on the column and when the sonication time was increased to 6 minutes, $\sim 33 \%$ of the sonicated material was retained on the column indicating a possible decrease in molecular size, but more likely do to volume loss as an aerosol during sonication.

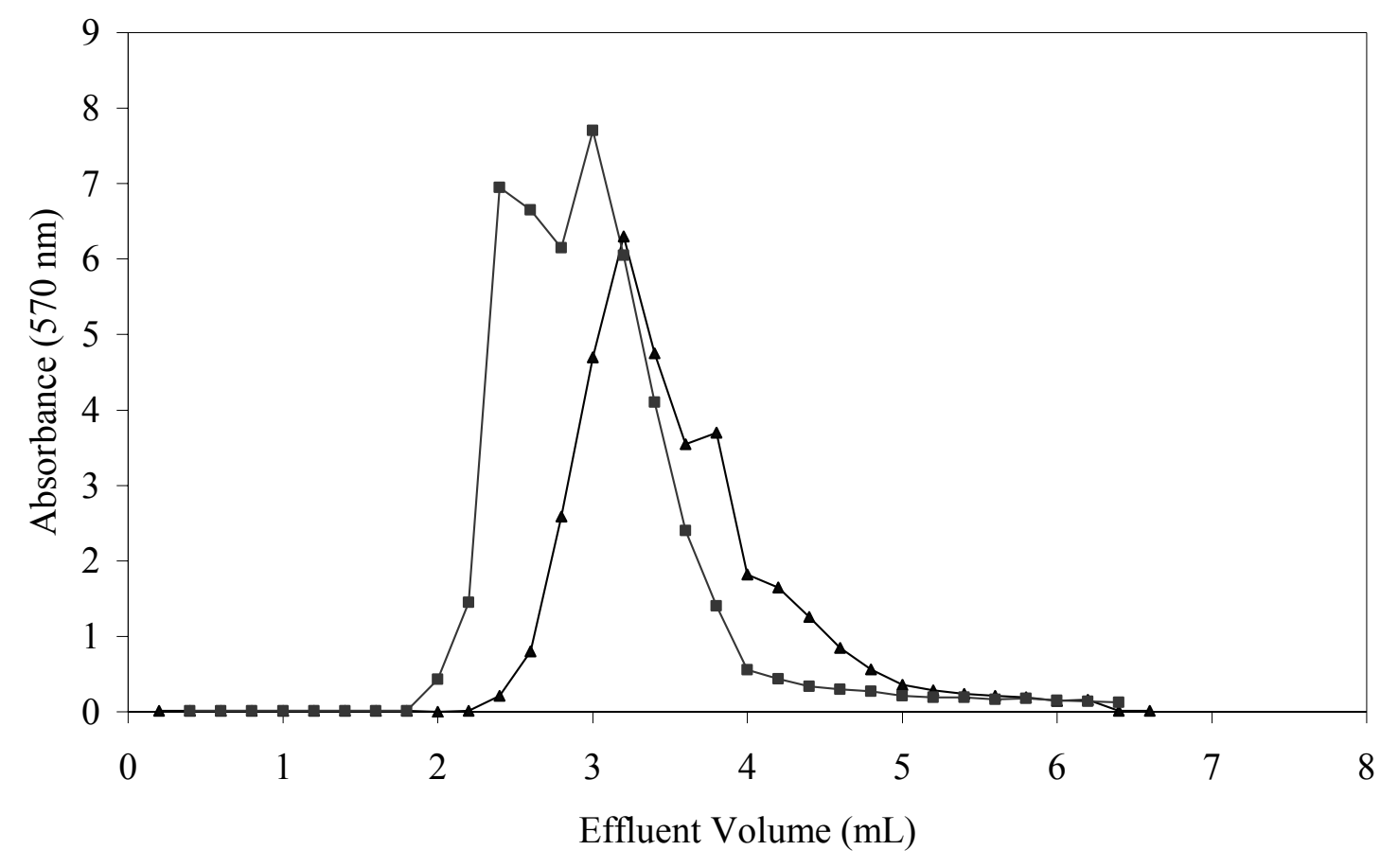

$\rightarrow$ Sonicated $\rightarrow-$ Non-sonicated

Figure 26. The distribution of bR following Sepharose gel filtration for a sample sonicated for 2 minutes at $30 \%$ power amplitude with a $0.2 / 2$ on/off pulse regime coupled with the non-sonicated sample. 


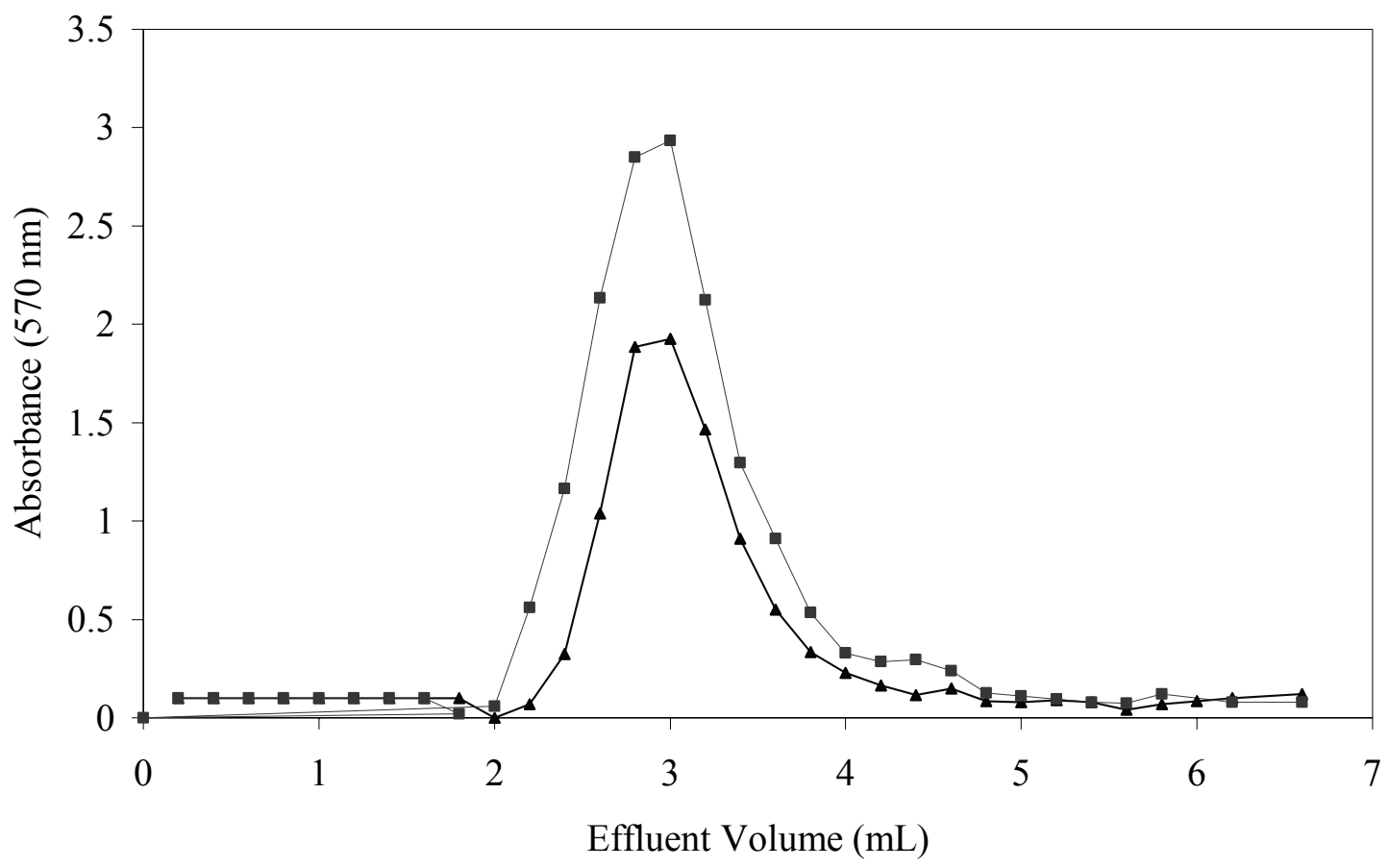

$\leftarrow$ Sonicated - Non-sonicated

Figure 27. The distribution of bR following Sepharose gel filtration for a sample sonicated for 6 minutes at $45 \%$ power amplitude with a $0.2 / 2$ seconds on/off pulse regime coupled with a non-sonicated sample. Note: the power amplitude total sonication time was increased but all other conditions were constant.

As discussed previously, if $\mathrm{A}_{280} / \mathrm{A}_{570}$ is $<<2$ or $>>2$, it is an indication of contamination, denaturation, or retinal dissociation. This parameter was calculated after each spectrometer measurement. During one sonication experiment, a program malfunction during sonication occurred causing the sample temperature to exceed $90{ }^{\circ} \mathrm{C}$. Since the purple color of the PM was maintained, the effect of over-heating was detected by the shift in the $\mathrm{A}_{280} / \mathrm{A}_{570}$ which increased to $>2.5$, and a shift in the peak at $570 \mathrm{~nm}$ indicating that the retinal had dissociated from the bR. This experiment mishap showed that $\mathrm{bR}$ in the aqueous state will denature with sonication and temperatures $>90^{\circ} \mathrm{C}$.

This experiment was repeated with closer monitoring of the sample during sonication. During the repeated experiment, the sample temperature never exceeded $25{ }^{\circ} \mathrm{C}$ at $45 \%$ power amplitude with a $0.2 / 2$ seconds on/off pulse regime. The PM was sonicated for 24 minutes and then evaluated using Sepharose $2 \mathrm{~b}$ gel size exclusion chromatography. A protein sample volume of $150 \mu \mathrm{L}$ was applied to the column. Aliquots were collected and measured using a Nanodrop spectrometer as shown in Figure 28. There was no appreciable difference between the sonicated and non-sonicated samples of PM from 6 minutes to 24 minutes of sonication. 


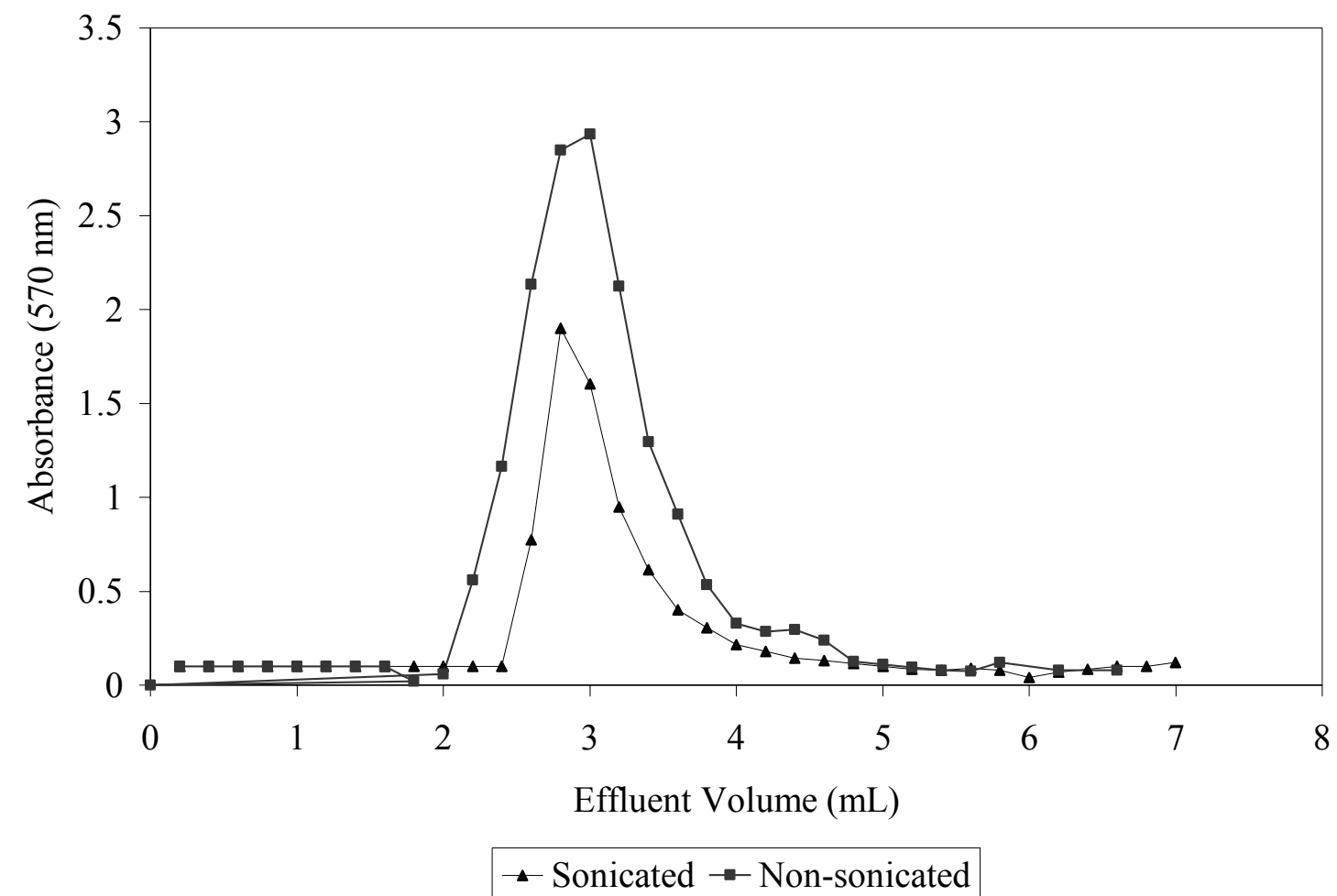

Figure 28. The distribution of bR following Sepharose gel filtration for a sample sonicated for 24 minutes at $45 \%$ power amplitude with a $0.2 / 2$ seconds on/off pulse regime and non-sonicated sample.

\subsection{Conclusions of Sonication}

In this study it was determined that sonication had limited success with PM patch reduction as demonstrated with a combination of sucrose gradient density centrifugation and size exclusion gel chromatography. The maximum reduction in patch size occurred at 2 minutes of sonication with $30 \%$ power amplitude and $0.2 / 2$ seconds on/off pulse regime.

\subsection{Monomerization}

Dencher and Heyn [71] were able to solubilize and separate the bR within the PM into monomer subunits while maintaining functionality by utilizing non-ionic detergents such as Octyl $\beta$-D-glucopyranoside (OG) and Triton-X 100. Octyl $\beta$-D-glucopyranoside has several key advantages: the absorbance spectra do not overlap; it does not fluoresce; and its critical micelle concentration (cmc) of $24 \mathrm{mM}$ was $\sim 100$ times higher than Triton X100 [72]. Dencher and Heyn [71] determined that when PM was solubilized with OG, $\mathrm{bR}$ monomers are formed in micelles with a Stokes radius of $2.8+/-0.5 \mathrm{~nm}$. This design constraint implies that the smallest possible gate pad could be $\sim 6 \mathrm{~nm}$ in diameter. 
In this research, multiple experiments were conducted to determine the optimal conditions for monomerization of PM, reducing the average diameter of the PM fragments from $500 \mathrm{~nm}$ and $5 \mathrm{~nm}$ thick to micelles which were composed of a few monomers $\sim 6.3 \mathrm{~nm} \times 6.3 \mathrm{~nm} \times 5 \mathrm{~nm}$. The solubilization of $\mathrm{PM}$ with an initial concentration of $11 \mathrm{mg} / \mathrm{mL}$ was suspended and aliquoted in 6 vials containing Octyl $\beta$-Dglucopyranoside concentrations ranging from 20 to $100 \mathrm{mM}$ with a $\mathrm{pH}$ of 6.9 , as shown in Table 1. Data on the six samples was collected every $6 \mathrm{hrs}$. Light exposure, temperature, vortex time and speed, sonication, and centrifugation were held constant.

Table 1. Initial values of concentration of $\mathrm{OG}$ and $\mathrm{bR}$.

\begin{tabular}{|c|c|c|}
\hline $\begin{array}{c}\text { Concentration } \\
(\mathbf{m M})\end{array}$ & $\begin{array}{c}\text { Initial Volume } \\
\text { (mL) }\end{array}$ & $\begin{array}{c}\text { Initial Mass } \\
\text { of bR (ug) }\end{array}$ \\
\hline \hline 20 & 4 & 880 \\
\hline 40 & 4 & 891 \\
\hline 60 & 4 & 1040 \\
\hline 70 & 4 & 1018 \\
\hline 80 & 4 & 880 \\
\hline 100 & 4 & 1046 \\
\hline
\end{tabular}

The data from the OG solubilization experiments are shown in Figure 29 - Figure 32. From the experiments, the time necessary to solubilize PM over a range of concentrations from $20 \mathrm{mM}$ to $100 \mathrm{mM} \mathrm{OG}$ is shown. The percent solubilization was determined by the amount of $b R$ that remained in the supernatant following refrigerated ultracentrifugation at $4^{\circ} \mathrm{C}$. If no pellet was formed after ultracentrifugation at $2 \times 10^{5}$ for 45 minutes, the bR was considered to be $100 \%$ solubilized. The percent solubilization was calculated by dividing the absorbance of the supernatant after ultracentrifugation by the absorbance of the supernatant prior to centrifugation. The percent denatured $b R$ was calculated at each incubation time $(\mathrm{t})$ by subtracting the initial absorbance of the stock after ultracentrifugation by the absorbance of the stock supernatant after ultracentrifugation at each incubation time $(\mathrm{t}=0,6,12,18$, and $24 \mathrm{hrs})$ this value was then divided by the initial absorbance of the supernatant after centrifugation. 
The detergent experiment (Figure 29 - Figure 32) also revealed that a range of OG concentrations from $60 \mathrm{mM}-100 \mathrm{mM}$ could solubilize in $<6$ hours with limited loss of bR. Quantified by $\mathrm{A}_{280} / \mathrm{A}_{570}$ of $\sim 2$, when values were $>2.5$ or $<1.5$ indicated poor quality or denatured $b R$. In the presence of OG, the solubilization of the bR started immediately as shown in Figure 29, while the $\mathrm{A}_{280} / \mathrm{A}_{570}$ begins to shift after $6 \mathrm{hrs}$ of incubation as shown in Figure 31. The data suggests that the good solubilization time was 6 hours with OG concentrations between $60-100 \mathrm{mM}$.

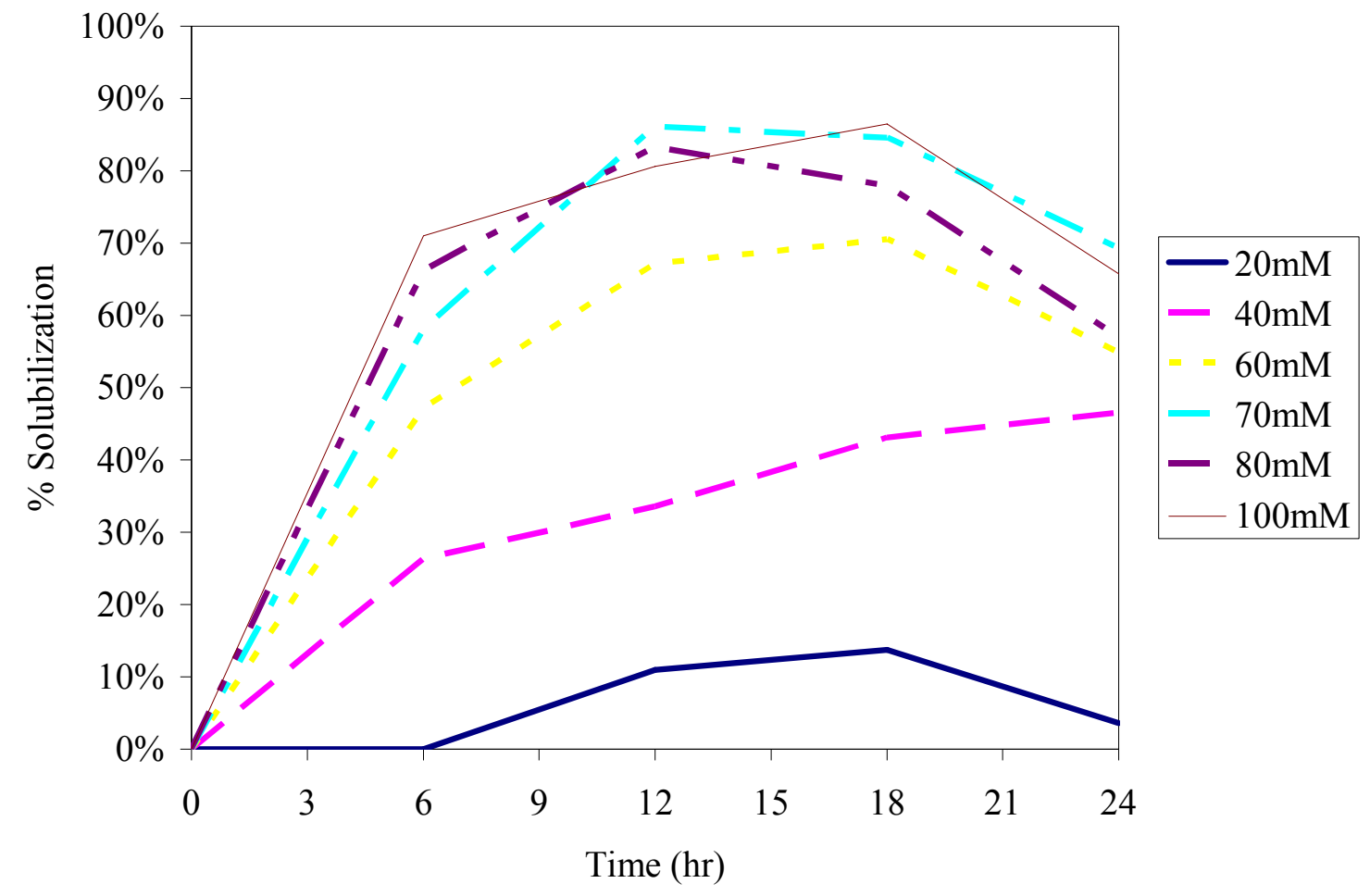

Figure 29. \% solubilization of $b R$ at different concentrations of $O G$ with respect to time. 


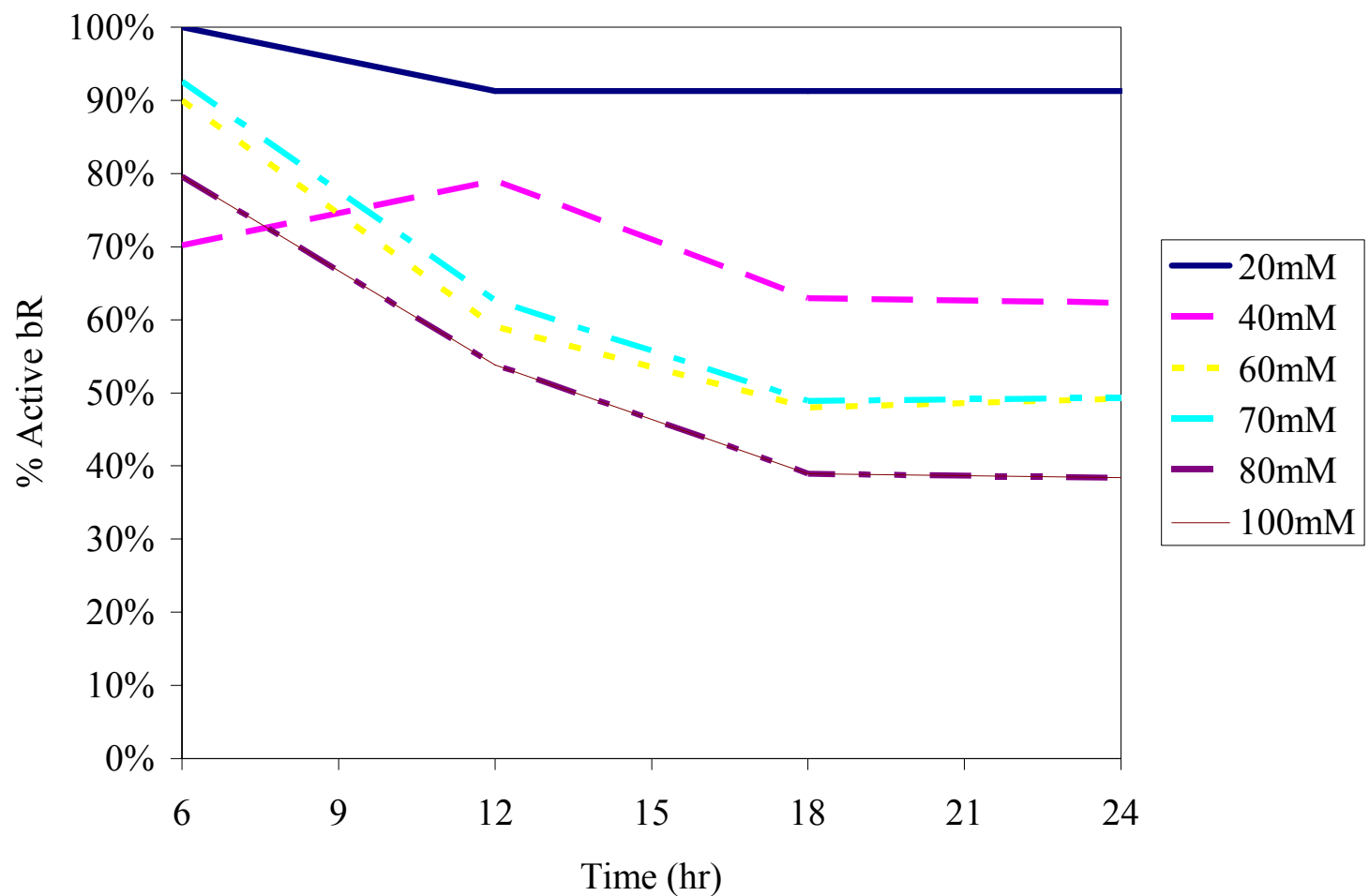

Figure 30. Calculated $\%$ active bR at different OG concentrations with respect to time.

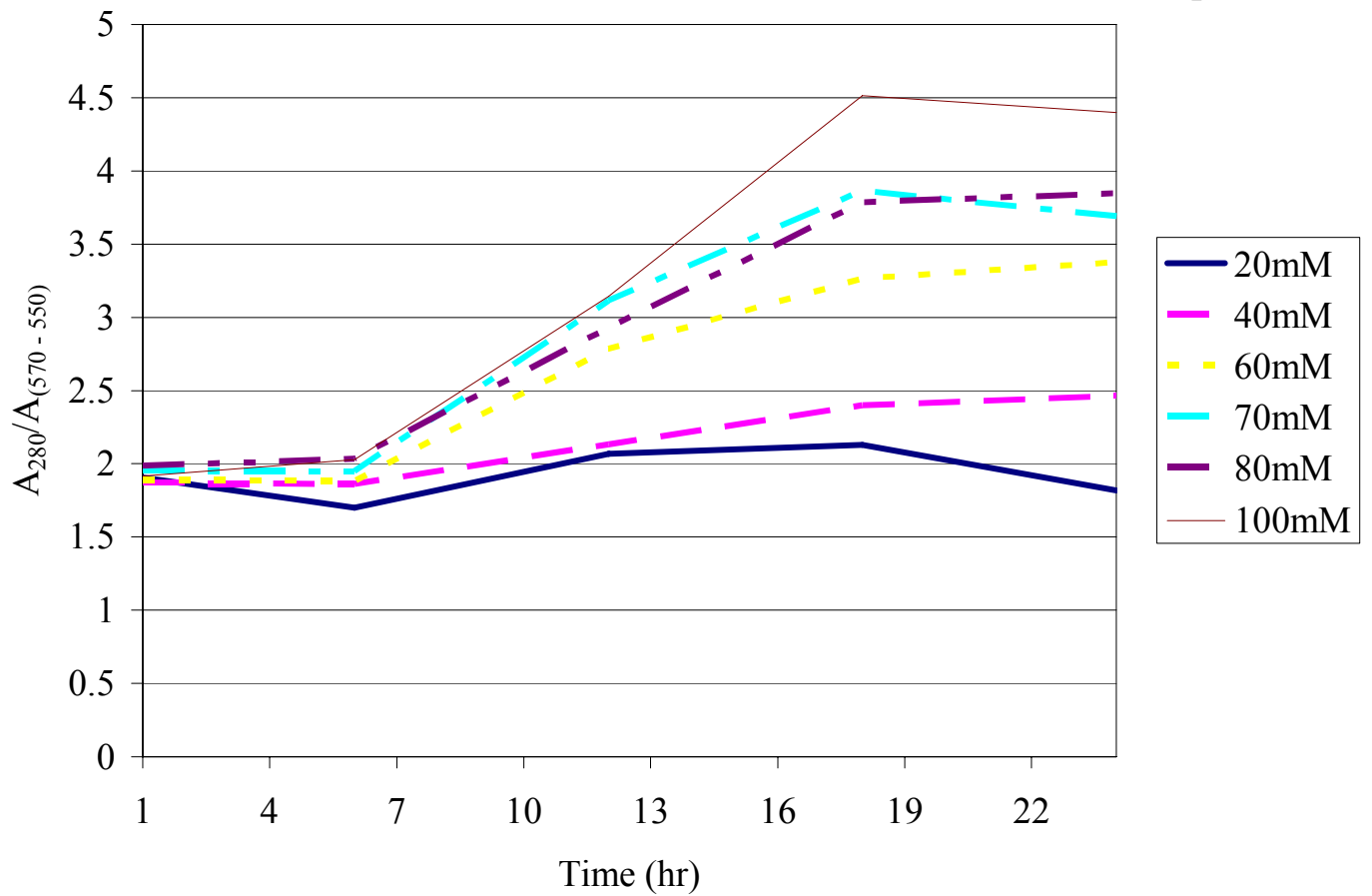

Figure 31. Pre-ultracentrifuge $\mathrm{A}_{280} / \mathrm{A}_{570}$ at $\mathrm{OG}$ concentrations from $20-100 \mathrm{mM}$ with respect to time. 


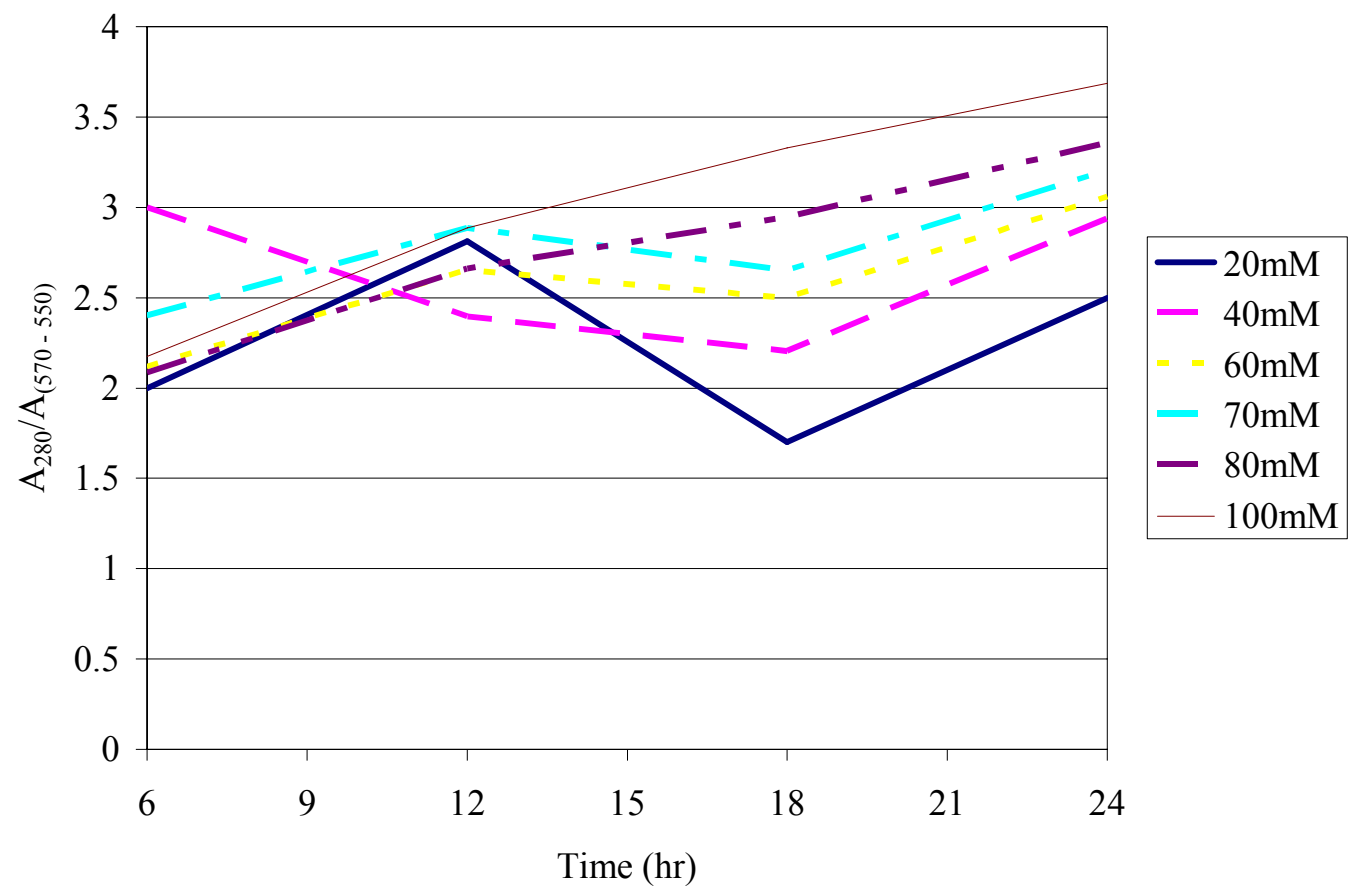

Figure 32. Post-ultracentrifuge $\mathrm{A}_{280} / \mathrm{A}_{570}$ at $\mathrm{OG}$ concentrations from $20-100 \mathrm{mM}$ with respect to time.

The effect of $\mathrm{pH}$ on the solubilization of $\mathrm{PM}$ in the formation of $\mathrm{bR}$ monomers was also evaluated. For this experiment the $\mathrm{pH}$ of a phosphate buffer solution was varied from $\mathrm{pH}$ 5.85 to 7.5 , while the concentrations of $\mathrm{bR}\left(0.25 \mathrm{mg} \mathrm{mL}^{-1}\right.$, and $\left.\mathrm{OG}, 70 \mathrm{mM}\right)$ were held constant. As before light exposure, temperature, vortex time and speed, sonication, and ultracentrifugation were held constant.

The results of this experiment suggest that the PM solubilization rate increased as the $\mathrm{pH}$ increased, as shown in Figure 33. The first 6 hours suggest the percent solubilization versus $\mathrm{pH}$ followed a linear trend. As the $\mathrm{pH}$ increased the percent solubilization increased, as shown in Figure 34. Since the absorbance ratios shown in Figure 35 and Figure 36 were $>2$, as a function of incubation time and $\mathrm{pH}$, these results suggest that $\mathrm{PM}$ solubilization should be conducted for under 6 hours at a $\mathrm{pH}<7.5$. These experiments also confirmed published observations by others that there was a shift in the major absorbance peak from $570 \mathrm{~nm}$ to $\sim 550 \mathrm{~nm}$ when $\mathrm{bR}$ was in the monomeric form $[71,72]$.

\subsection{Monomerization Conclusions}

From these experiments, it was determined that OG monomerizies PM, and bR monomer isolation parameters were selected that maximize the percent solubilization of active $b R$. The optimal parameters include an incubation time of $6 \mathrm{hrs}$, a $\mathrm{pH}$ of 6.9 , and an OG concentration of $70 \mathrm{mM}$. 


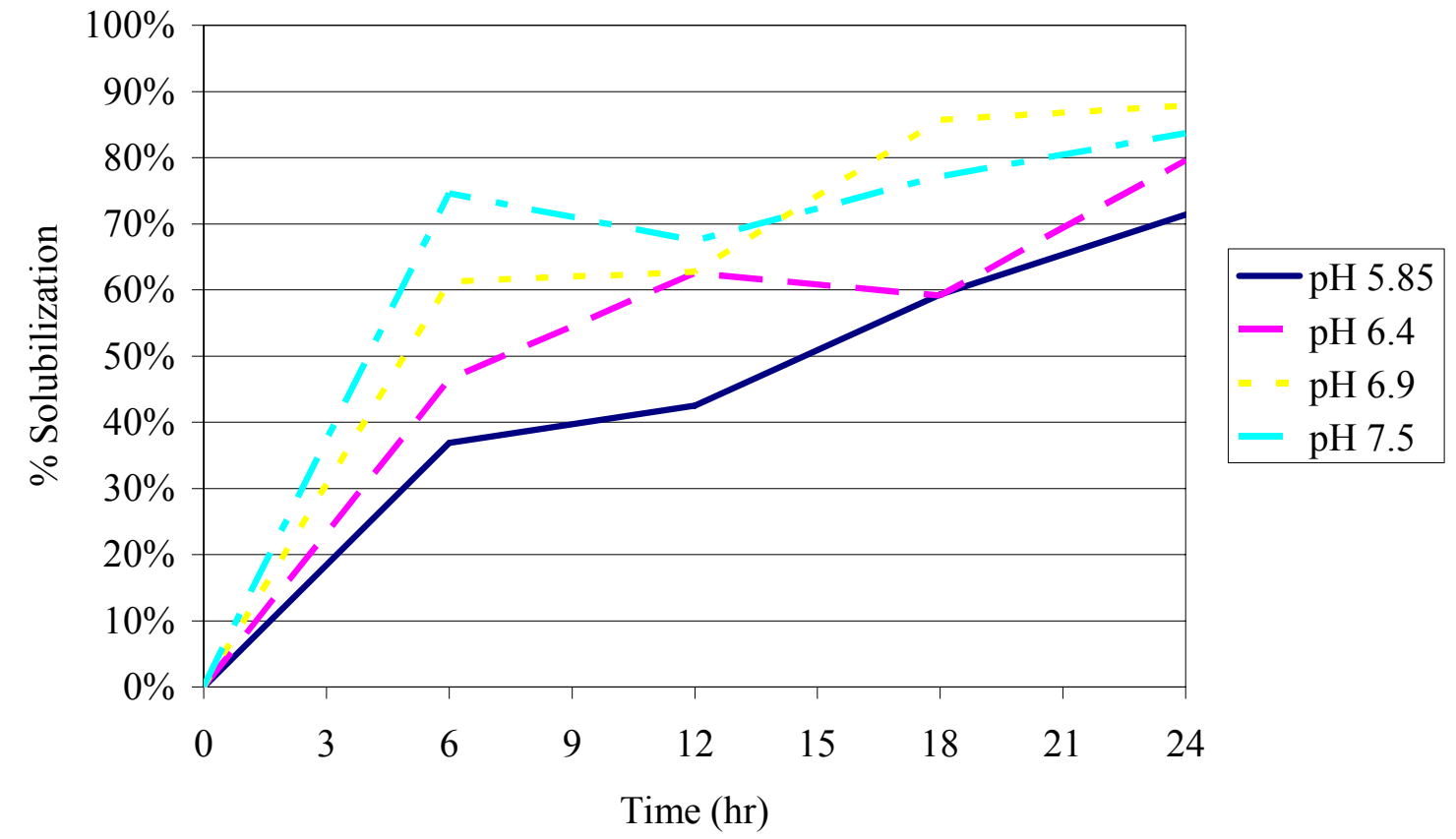

Figure 33. Percent solubilization of $\mathrm{bR}$ versus time at various $\mathrm{pH}$.

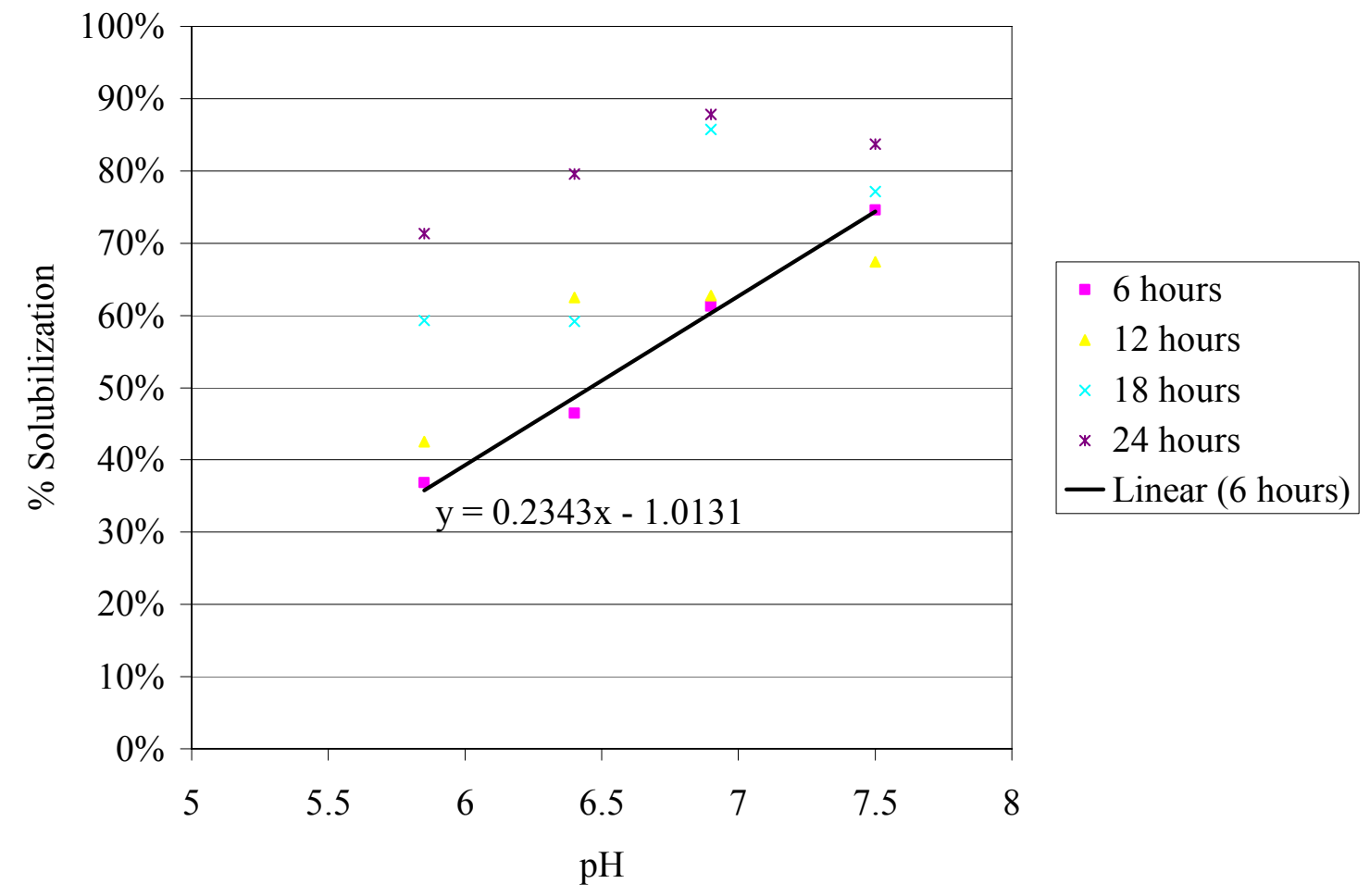

Figure 34. Percent solubilization of $\mathrm{bR}$ versus $\mathrm{pH}$ at various times. 


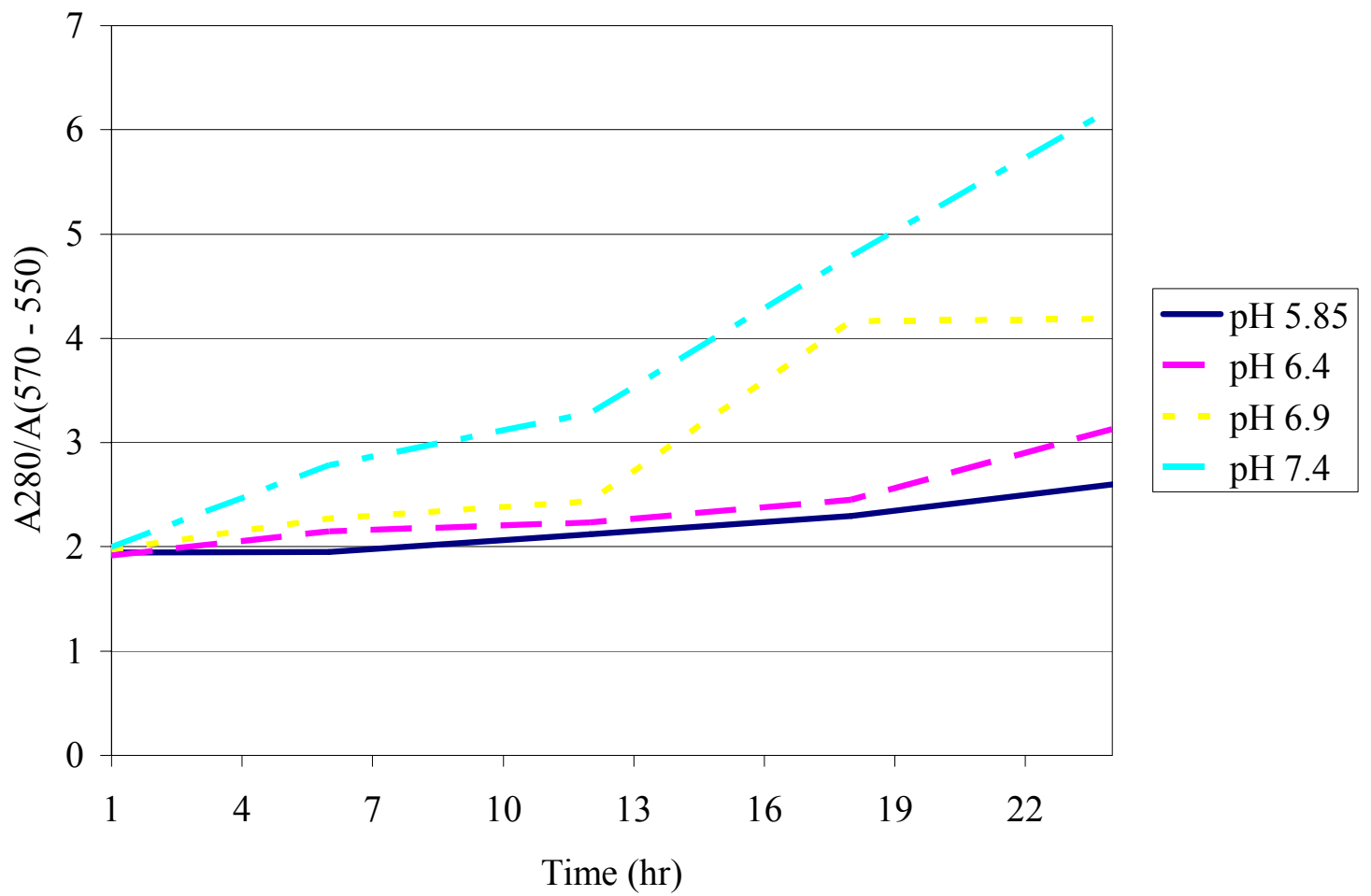

Figure 35. Pre-centrifuge $\mathrm{A}_{280} / \mathrm{A}_{570}$ with respect to time and $\mathrm{pH}$.

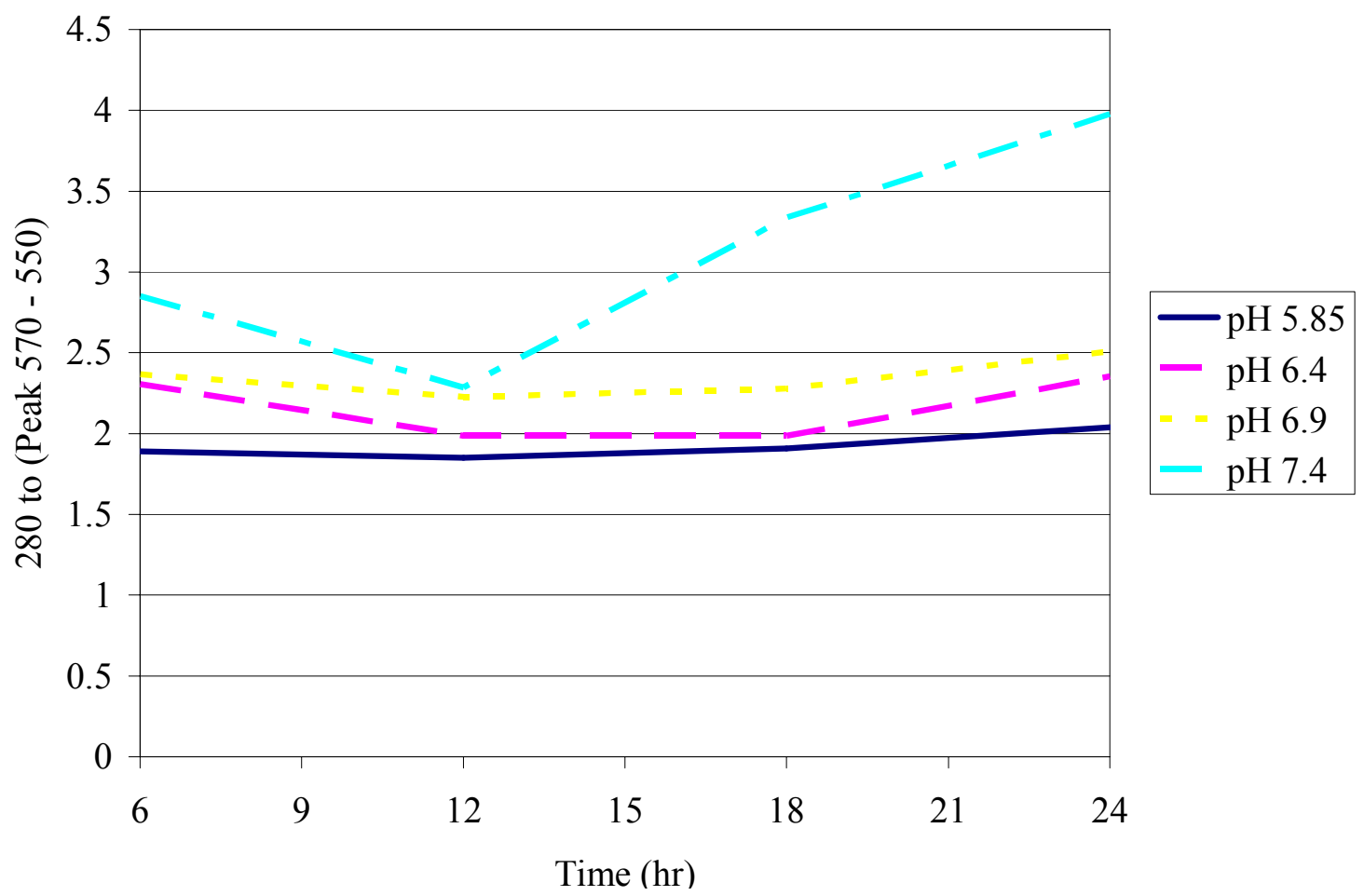

Figure 36. Post-centrifuge $\mathrm{A}_{280} / \mathrm{A}_{570}$ with respect to time and $\mathrm{pH}$. 


\section{Chapter 5 Biotinylation, Deposition, and Activation}

Depositing and verifying the photovoltaic response of bR became routine for this research. Knowing the voltage output of the bR was necessary to determine if activation of a SET was possible. The simplest method of depositing bR was by electrophoretic deposition (EPD), as described earlier. Through preliminary research it was determined that to preferentially orient the bR with EPD the bR solution must be buffered to create a dipole, the extracellular side being more negative. The buffer was a $1 \mathrm{mM}$ potassium phosphate with a $\mathrm{pH}>6.5$. With the bR buffered prior to EPD, the photovoltage response of the dried bR was 10 to 100 times larger than without, indicating that the orientation of the bR was greatly improved. Buffering the bR solution and subphase were also important for depositing and orienting LB layers of $b R$.

\subsection{Biotinylation of $b R$}

The ability to control the orientation of bR molecules is important during monolayer deposition. The biotin-streptavidin combination described earlier can be used to achieve this control. In this set of experiments bR was biotinylated and the degree of biotinylation was quantified. The specific biotinylation of bR at lysine 129, was accomplished chemically by raising the $\mathrm{pH}$ of the buffer to $\sim 9$ and adding biotin to the buffered bR solution. By raising the $\mathrm{pH}$ to $\sim 9$ the only available binding site is lysine 129 on the extracellular surface of bR, as previously shown in Figure 12. The biotinylated-bR was then evaluated by both SDS PAGE, and the 4-Hydroxyazobenzene2carboxylic acid/Avidin (HABA/Avidin) assay. As shown in SDS-PAGE gel Figure 18, the slight upward shift of the biotinylated $b R$ band compared to the $b R$ band suggested that biotin was attached to bR. The (HABA/Avidin) assay was the final procedure used to determine the degree of biotinylated $\mathrm{bR}$.

HABA/Avidin assays of biotinylated $\mathrm{bR}$ were prepared to determine the amount of biotin bound to the bR. HABA is a dye which binds to Avidin. When bound to Avidin, the HABA absorbs light at a wavelength of $500 \mathrm{~nm}$. However, when the HABA $\backslash$ Avidin solution is combined with biotin, the biotin displaces the HABA causing the absorbance at $500 \mathrm{~nm}$ to decrease. So, the molar concentration of biotin can be determined based on the amount of HABA displaced. A standard curve was generated for the HABA/Avidin assay using known levels of biotin [73], shown in Figure 37. Using this absorbance versus molar concentration standard curve, the molar concentration ratio of biotin bound to bR was determined and is represented by the circled dots in Figure 37. These sample ratios agree well with each other, and these results were similar to those published in Chen et al. [74]. 


\subsection{Conclusions of Biotinylation of $\mathrm{bR}$}

The molar ratios indicate that within each PM patch about 1 in every 2 bR's were biotinylated. There were hundreds of thousands of bR molecules within each PM, so this indicates that the biotinylated bacteriorhodopsin can be used to control the orientation of the molecule. This could be useful for depositing highly oriented single monolayer of bR on a device and a single monolayer does seem desirable for sensor applications.

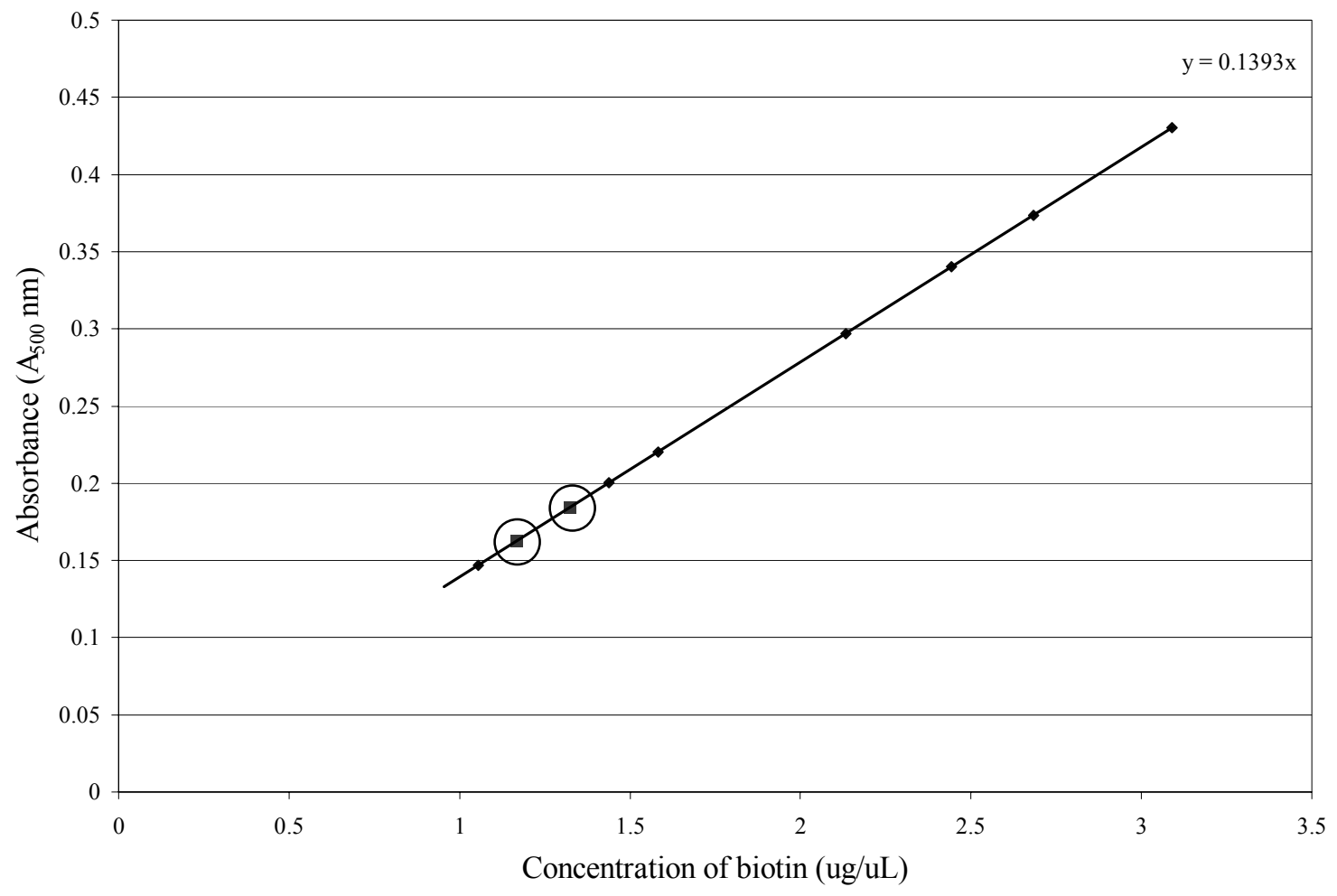

Figure 37. Biotin - HABA/Avidin standard curve. Change in absorbance at $500 \mathrm{~nm}$ of HABA/Avidin reagent with the addition of biotin. Biotinylated-bR is represented by the circled square symbols.

\subsection{Photoelectric Response of bR Films}

Experiments were conducted to investigate and become familiar with methods of immobilizing and orienting $\mathrm{bR}$ on a transducing substrate and then measuring its photoresponse. This will be useful information when developing a bR-based chemical/biological detection device. Two methods were investigated to immobilize and orient bR onto a conductive ITO electrode surface; 1) electrophoretic deposition and 2) the use of a Langmuir-Blodgett trough. Initial results were promising with the selected methods. The next step was to improve the photoresponse and repeatability. As a control, bare ITO was tested, resulting in no detectable photoresponse. Figure 38, shows 
the emission spectrums of the photon sources used with respect to the absorbance spectrum of $b R$.

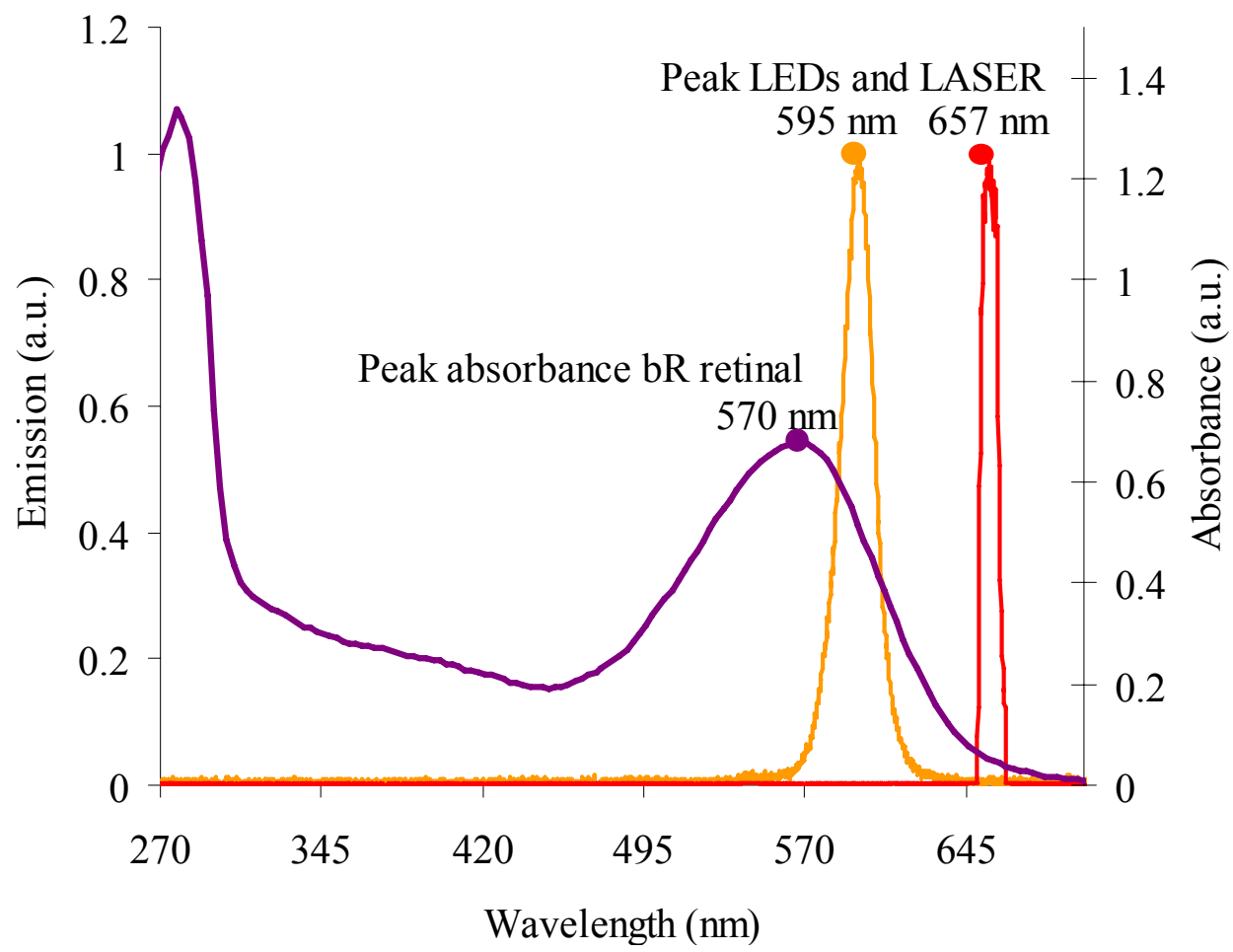

Figure 38. Absorbance of $\mathrm{bR}$ and the emission spectrums of amber LEDs and red Laser pointer.

\subsection{Measurement Methods}

Measuring the photoresponse of bR was not a trivial task as it required a high impedance instrument capable of measuring extremely small charges effects. One such device is an electrometer. Another is a high impedance operational amplifier (op-amp). The high impedance op-amps offer electrometer like sensitivity in the form of a chip and at a fraction of the price of an electrometer. For these reasons high impedance op-amps were the chosen tool to measure he photoresponse of $b R$. Using a high impedance operational amplifier multiple printed-circuit-broads (PCB) were designed, tested, and refined till the bR signal could be measured repeatedly. A couple of the working PCBs are described below.

To initially measure the photoresponse, the circuit design utilized a high impedance opamp (LMC 6001) from National Semiconductor Corporation. As seen in Figure 39 the initial circuit consisted of two LMC 6001 operational amplifiers, the non-inverting amplifier configuration was used for measurements and the inverting amplifier configuration was used as a buffer (in theory) which would help stabilize and zero the 
inherent offset in the measurement op-amp. This circuit design was similar to that of Wang et al. [75]. In practice this design was not stable. With this design the circuit was extremely unstable when connected to the bR photocells. It was extremely hard to bring the circuit out of saturation.

In an attempt to improve the stability of the circuit and reduce the complexity of the PCB design a single op-amp LMC 6061, by National Semiconductor Corporation in a noninverting configuration was used. As shown in Figure 40 this PCB design reduced the number of components, fabrication, and cost by about half. This design was more stable then the last PCB design and the photovoltaic response of bR could be measured repeatedly. The circuit was battery powered by a balanced battery power supply as shown in Figure 40 and enclosed in a Faraday cage to reduce noise. And one other trick used to noise and leakage associated with the chip the and broad, the op-amps input pin (pin 3) was bent away from the PCB and soldered in the air and connected to the bR with the shortest lead length.

The gain in the circuit shown in Figure 40 was controlled by changing the ratio of resistors in the non-inverting configuration. For this work the gain was set between 1 and 11. A low pass cutoff filter set at $30 \mathrm{~Hz}$ with a gain as high as 100 could be introduced at the output of the high impedance op-amp to increase the gain to 1100 and reduce noise. The output signal from the op-amp or cutoff filter was then inputted into a digital oscilloscope.

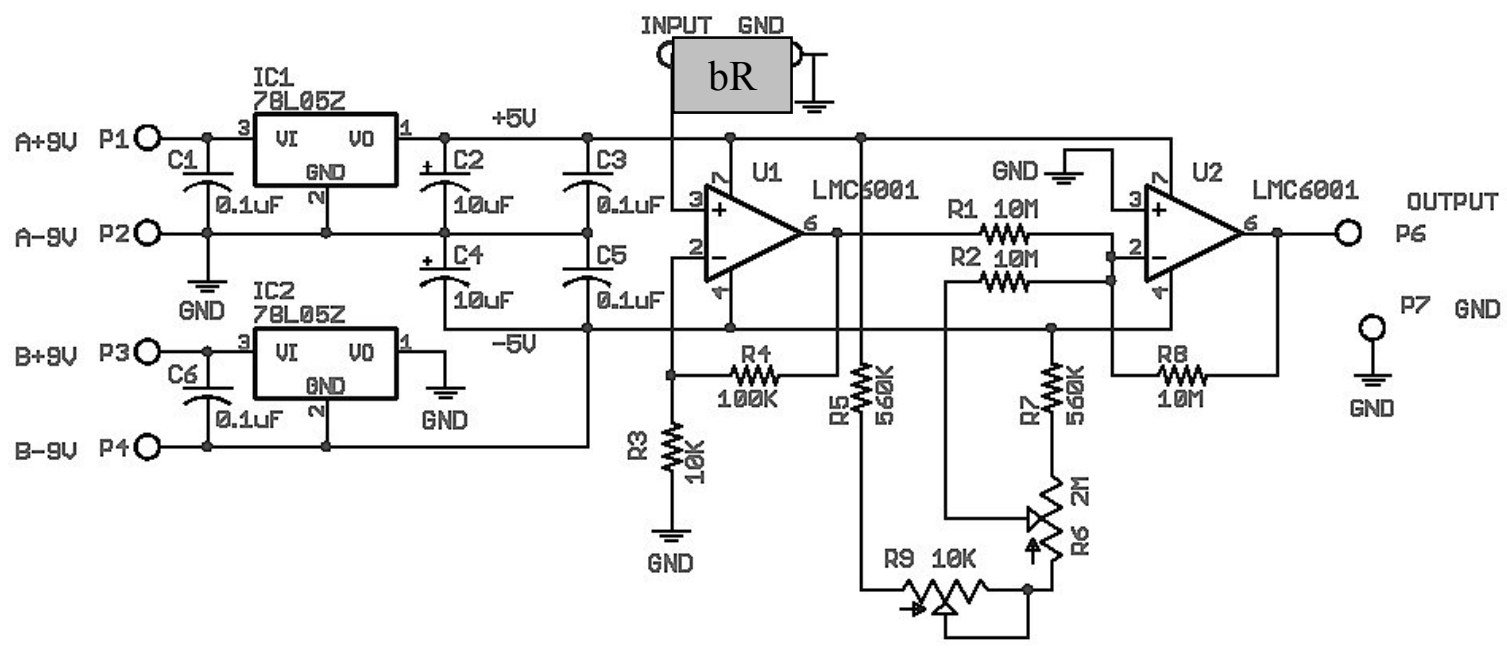

Figure 39. Schematic of initial measurement circuit. 


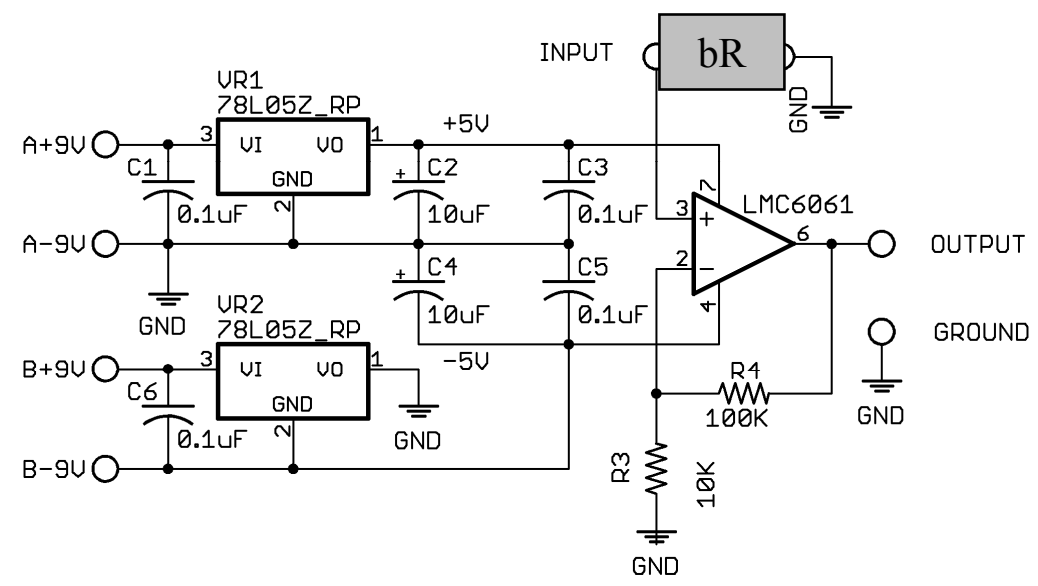

Figure 40. Final circuit schematic.

Another method of measuring electrical properties of bR layers is with the Keithley 4200 Semiconductor Characterization System. Both measurement systems were utilized in this research.

\subsubsection{Electrophoretically Deposited bR}

Electrophoretic deposition is a method utilizing a voltage potential that is applied across a solution containing a dipole molecule, bR, to deposit and orient on the anode. Prior to electrophoretic deposition the bR concentration was adjusted to $5-15 \mathrm{mg} \mathrm{mL}^{-1}$ in DDI water. It was determined that $\mathrm{bR}$ would orient better if it was suspended in a buffered solution of $0.75 \mathrm{mM} \mathrm{KH}_{2} \mathrm{PO}_{4}$ with a $\mathrm{pH}$ of 6.9 . This increased the voltage response 10 to 100 times. During electrophoretic deposition the gap separating the positively charged ITO electrode and negatively charged gold electrode was $\sim 1.5$ millimeters and arranged so the anode was on the bottom. A voltage of $4 \mathrm{~V}$ was applied for 45 seconds. The bR ITO substrates were then dried in a humidity chamber with $\sim 55 \% \mathrm{RH}$. The bR films were dried to increase durability.

\subsubsection{ITO Top Electrode}

Bacteriorhodopsin photocells were constructed by first electrophoretically depositing bR onto ITO and then sandwiching the bR with a counter ITO electrode, creating the bR photocell. Figure 41 shows a representative photovoltaic response of bR sandwiched between ITO. 


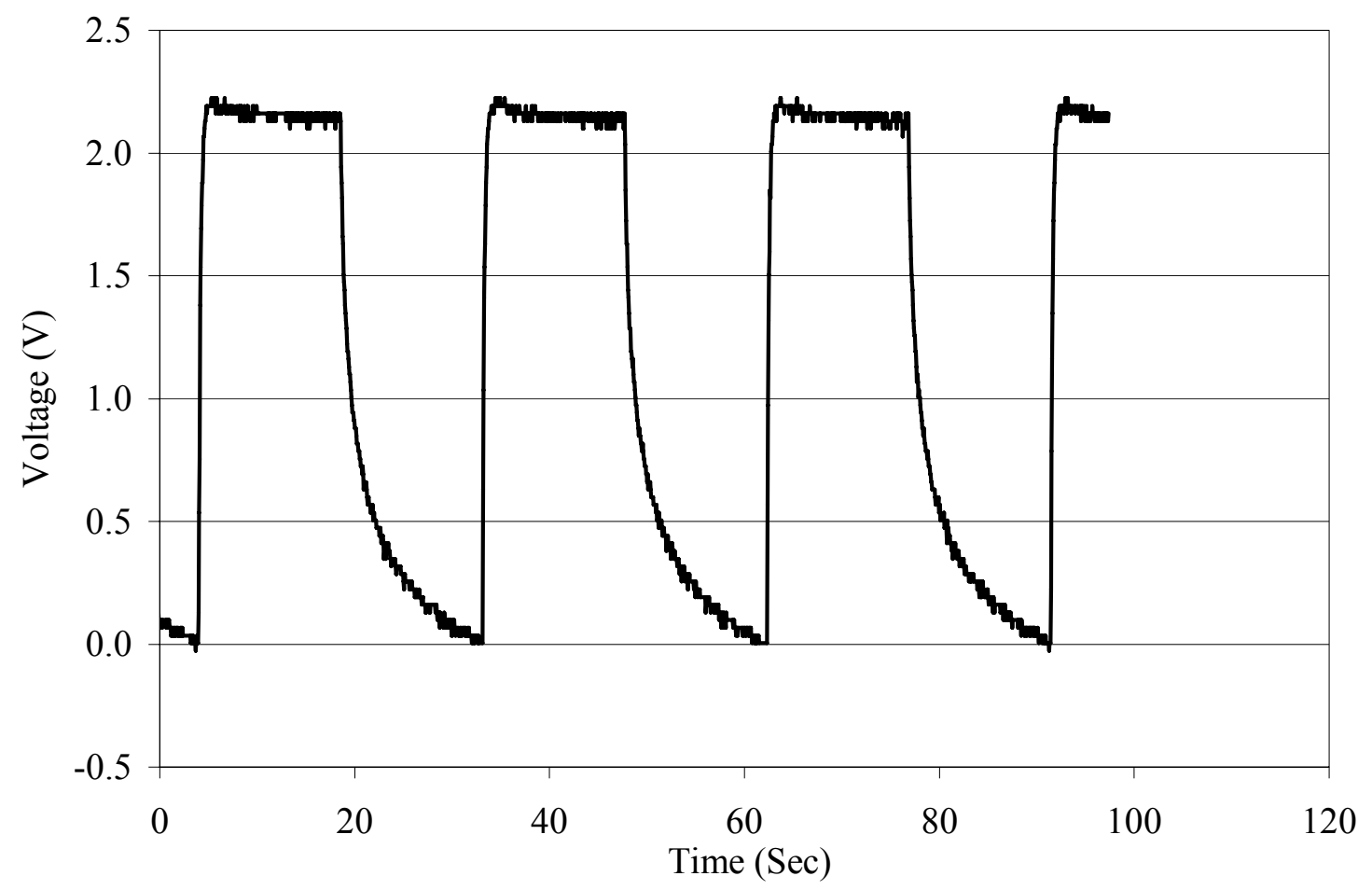

Figure 41. Representative photovoltaic response of bR sandwiched between ITO.

\subsubsection{Electrolyte Top Electrode}

A nondestructive top electrode was investigated to try to improve the photoresponse. For this experiment an electrolyte gel served as the top electrode. The electrolyte gel was prepared from a $1.5 \%$ weight percent Agar dissolved in $0.1 \mathrm{mM}$ potassium chloride $(\mathrm{KCl})$ solution with a $\mathrm{pH}$ of 5.4. The Agar electrolyte solution was then autoclaved for 0.5 hours to sterilize and cooled to room temperature prior to application.

Prior to cooling, the electrolyte gel was pipetted onto the immobilized bR creating a top electrode and completing the $b R$ photocell. To measure the photoresponse of $b R$ with a top electrolyte gel photocell, the bR photocell was connected to the circuit in Figure 39 as shown in Figure 42. The bR photocell was then flashed with light and a photoresponse was measured as shown in Figure 43. The camera flash had a wide range of photonic output power at $570 \mathrm{~nm}$ as shown Figure 44, which influenced the photoresponse. The shape of the photoresponse was comparable to the ITO-bR-ITO, just smaller in magnitude. There were many possible reasons for this difference: variation in the photonic power of camera flash; the electrolyte gel absorbance at $570 \mathrm{~nm}$; the angle of activation; leakage currents within the measurement circuit; variation in deposited bR; variation in the test environment; and variation in $\mathrm{pH}$ as reported in [38]. Using the electrolyte gel as a top electrode could be ideal because it can be patterned at room temperature, it is easily applied, inexpensive, and immobilized bR films are not be destroyed with the removal of a moist top electrode. 


\section{Circuit}

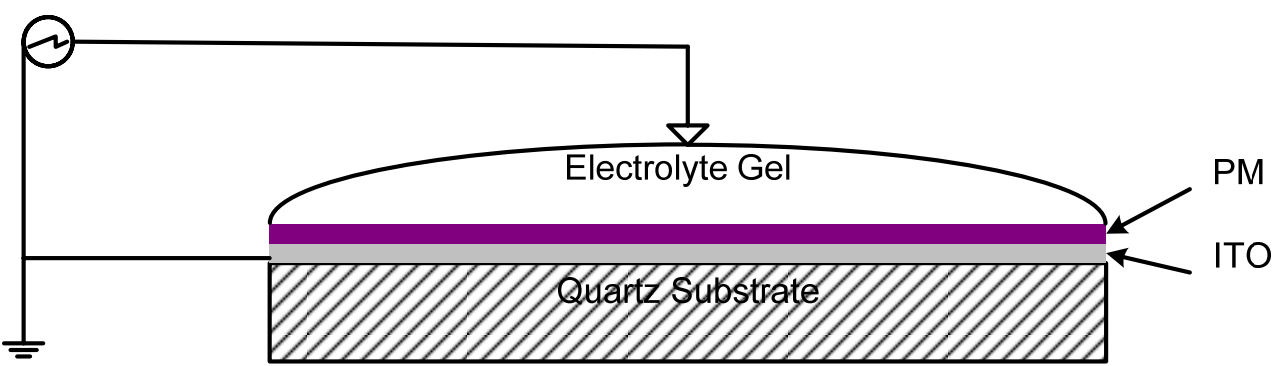

Figure 42. Simplified setup of the photoelectric measurement of $b R$.

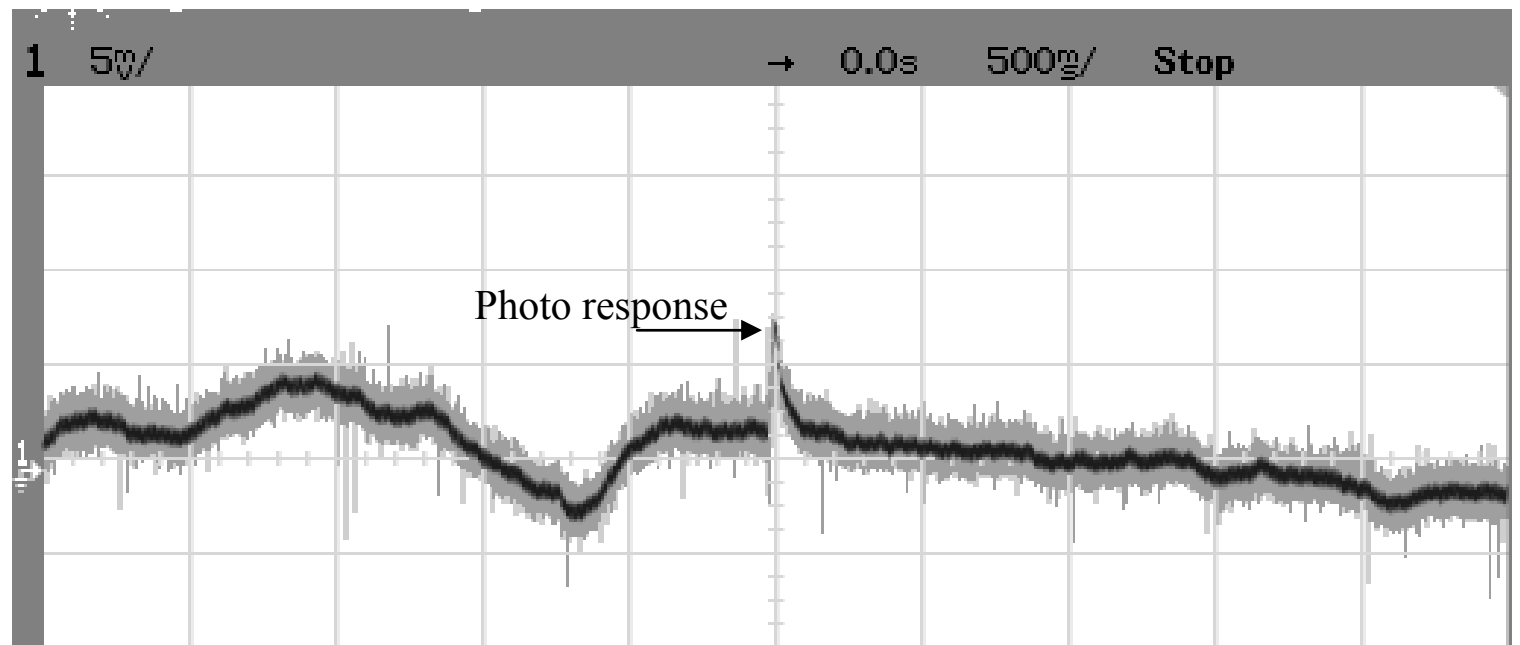

Figure 43. Photoresponse ( $\sim 5 \mathrm{mV}, 11 \mathrm{x}$ amplification) of electrodeposited $\mathrm{bR}$ films with an ITO bottom electrode and electrolyte gel top electrode.

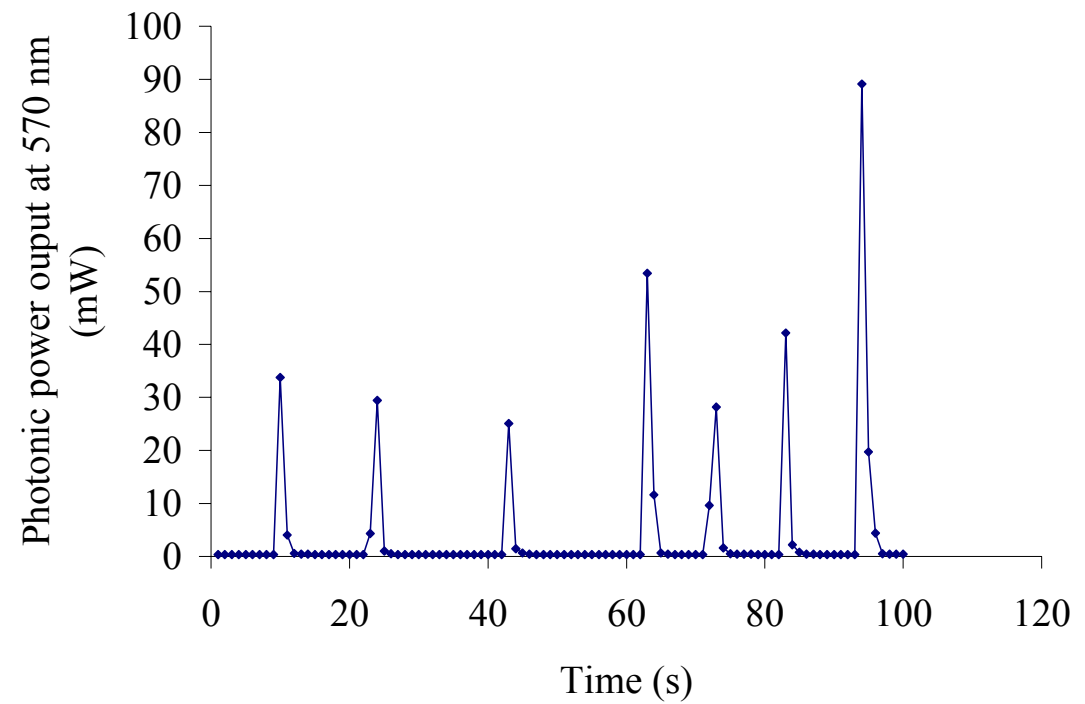

Figure 44. Measurements of the photonic power of the camera flash through a fiber optic cable at $570 \mathrm{~nm}$. 


\subsubsection{Gold Top Electrode}

Using the same $b R$ deposition procedures and the same concentration of $b R$, the photoelectric response was measured between two electrodes, one indium tin oxide (ITO) and the other sputtered gold $5 \mathrm{~nm}$ thick, as depicted in Figure 45.

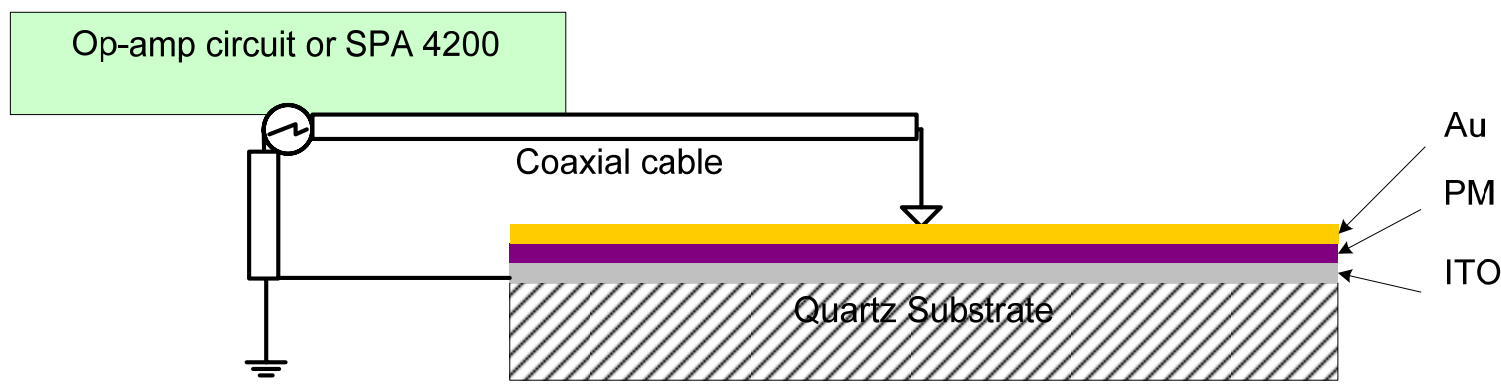

Figure 45. Setup of the photoelectric response measurement.

On the dried bR film, a mask was created using electrical tape with $3-4 \mathrm{~mm}$ diameter holes cut into the tape. The bR was then sputtered with $5 \mathrm{~nm}$ of Au under high vacuum in the Perkin Elmer 2400-6J RF sputter deposition system.

The large light transmittance and conductivity properties of thin gold layers (i.e. $<10 \mathrm{~nm}$ ) were ideal for this application where a stable (non-reactive) and transparent conductive layer was needed to form the top electrode on bR. A $5 \mathrm{~nm}$ layer of gold will transmit more than $60 \%$ of the incident light at $570 \mathrm{~nm}$ [76]. The photoresponse of the bR with a gold sputtered top electrode was measured by connecting them as shown in Figure 45 . Amber LEDs were the photon sources and resulted in the photoresponse shown in Figure 46 and Figure 47. As shown in these figures, the current response and the voltage response have different shapes. The difference in shape is due to the charge movement and charge displacement. Current is the movement of charge and within dried films of bR this occurs the instant the light is either turned on or off, as shown in Figure 46. Once the charge has been displaced a voltage potential will be measured till the light is turned off. 


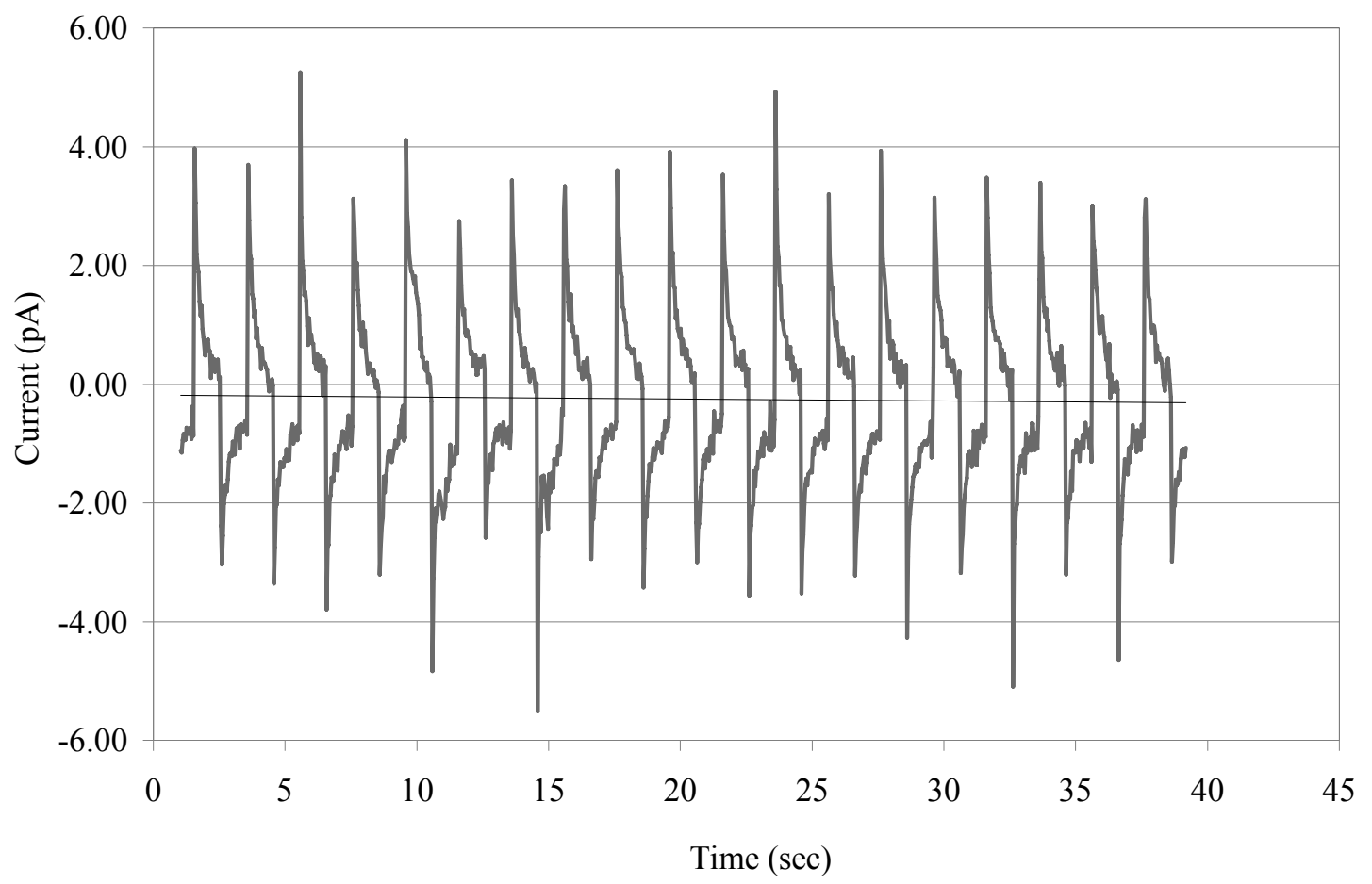

Figure 46. Current measurement of photon activated $b R$ with gold sputtered top electrode measured with the SPA 4200.

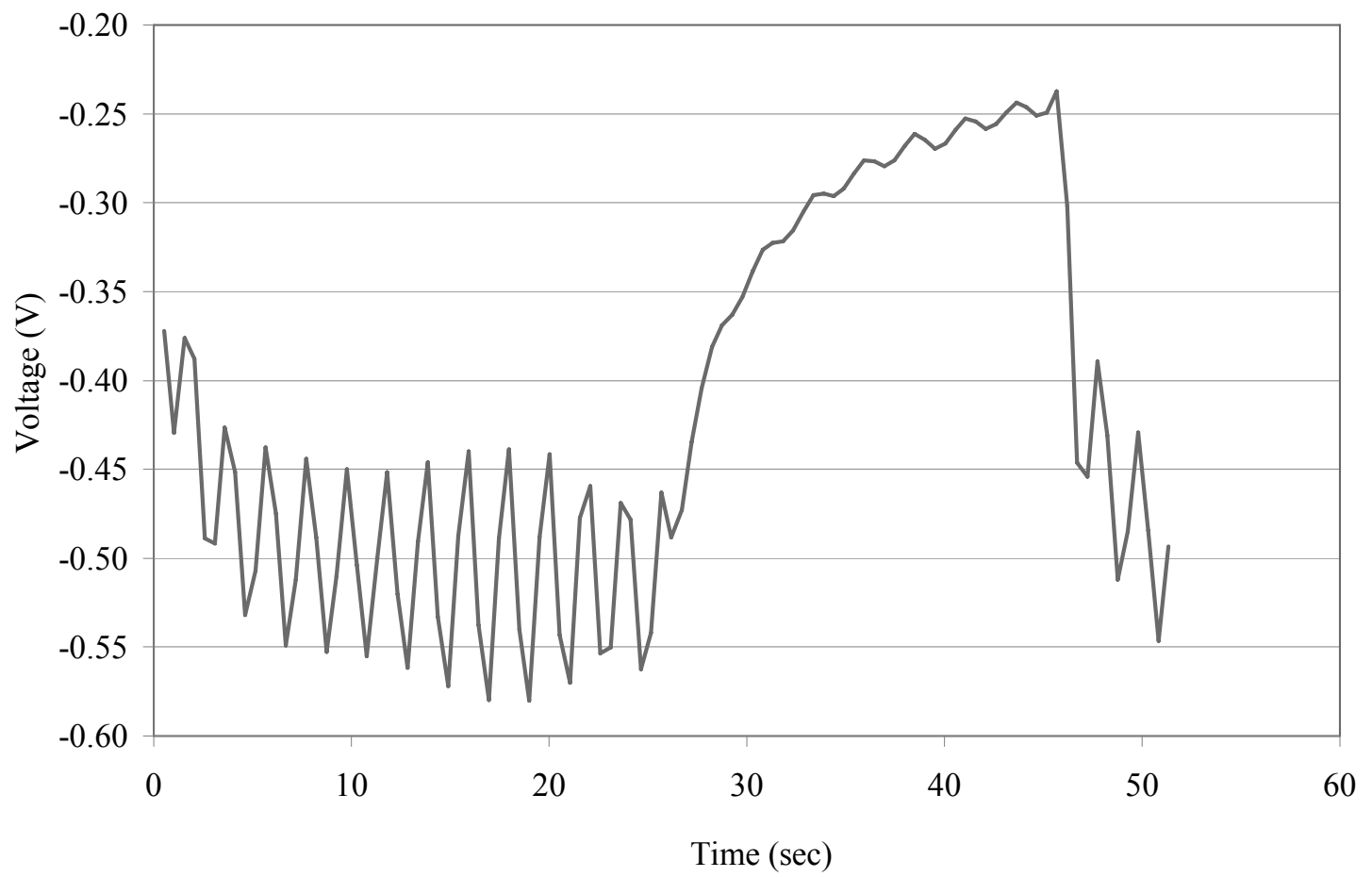

Figure 47. Unamplified photovoltaic response of $b$ at various flash rates with an ITO bottom electrode and sputtered gold top electrode measured with the SPA 4200. 


\subsubsection{Langmuir-Blodgett}

Using the LB technique, multiple monolayers were deposited on a conductive substrate and then qualified by the transfer ratio, the linear decrease of the area of the monolayer on the subphase, and the photoresponse as shown in Figure 50 and Figure 51. This ratio was defined as the area of the film transferred to the moving surface in comparison to the area of the film that was removed from the air-water interface [77] and should be $\sim 1$. The LB deposition method could be useful if $<10$ monolayers of $b R$ are needed to activate the SET.

The bR suspension was prepared by following the method developed by Hwang et al. [35], briefly outlined; 1) $\sim 1 \mathrm{~mL}$ of chilled hexane, 2) $\sim 0.5 \mathrm{~mL}$ of $0.2-0.8 \mathrm{mg} \mathrm{mL}^{-1} \mathrm{bR}$ suspended in either DDI water or a buffer solution with a $\mathrm{pH} \sim 7$, or 3$) \sim 0.5 \mathrm{~mL}$ of $0.2-$ $0.8 \mathrm{mg} \mathrm{mL}^{-1}$ bR:soya-phosphtidylcholine (soya-PC) (weight ratio 4:1 - 10:1). The phosphtidylcholine was added because it was thought to increase the orientation and conductivity of the dry film at the air/water interface. The bR:hexane solution was then emulsified by sonication for 5 seconds with a pulse regime of $0.2 / 2$ seconds on/off in an ice bath. The emulsion was then centrifuged for $10 \mathrm{~min}$ at 1,500 $\mathrm{g}$ creating a 3-phase solution, as shown in Figure 48. After centrifugation, the DDI water was removed with a Pasteur pipette leaving $\mathrm{bR}$ and hexane. The bR:hexane suspension was then brought to the desired concentration of $0.15-0.8 \mathrm{mg} \mathrm{mL}^{-1}$ by either adding or removing hexane. Then, just prior to spreading the bR:hexane suspension, it was vigorously mixed by brief sonication ( $1 \mathrm{sec}, 30 \%$ power $0.2 / 2.0$ seconds on/off). The bR:hexane, generally $200-250$ $\mathrm{mL}$ at a concentration of $0.35 \mathrm{mg} \mathrm{mL}^{-1}$, was then immediately spread onto the subphase at a rate of $50 \mu \mathrm{L}$ every one to two minutes until the surface pressure started to increase.

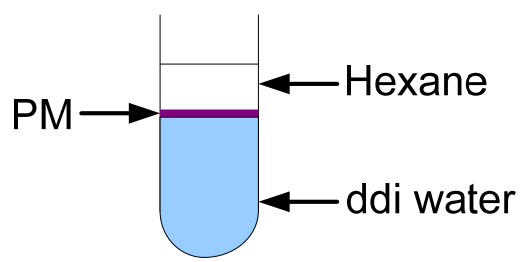

Figure 48. Post centrifugation of PM-Hexane suspension.

If too much was applied or the concentration was too large, clumping and stacking of monolayers occurred on the subphase [26]. Shortly after spreading the bR:hexane or bR: soya-PC:hexane suspension, the hexane evaporated leaving the bR or bR:soya-PC at the air/water interface of the subphase. The bR or bR:soya-PC was compressed to a surface pressure of $25-40 \mathrm{mN} \mathrm{m}^{-1}$ and compressed at a rate of $5-15 \mathrm{~mm} \mathrm{~min}^{-1}$ as mentioned by Boucher et al. [31]. Once the bR monolayer had equilibrated at the desired surface pressure, the substrate deposition process commenced.

Monolayers of bR were deposited onto the ITO coated quartz slide during the upward stroke at a rate of $5 \mathrm{~mm} \mathrm{~min}{ }^{-1}$ at a surface tension of $25-40 \mathrm{mN} \mathrm{m}^{-1}$. Each layer deposited was air-dried for $15-20$ minutes prior to the next monolayer deposition. The 
deposition was conducted in an enclosure to reduce dust and ventilation drafts. As shown in Figure 49, a total of 30 depositions were attempted but the only time that bR deposited on the ITO was during the upward stroke. So, a total of 15 monolayers were deposited, shown in Figure 49 as the odd numbered depositions.

Following the multi-monolayer deposition, the surface was coated with $5 \mathrm{~nm}$ of gold to form the top electrode. The photoresponse from the monolayers of $b R$ is shown in Figure 50 and Figure 51. The reproducibility of the photoresponses was investigated since it could have been influenced by a number of factors such as RF noise, photonic noise from the flash, and induced signal in the wiring.

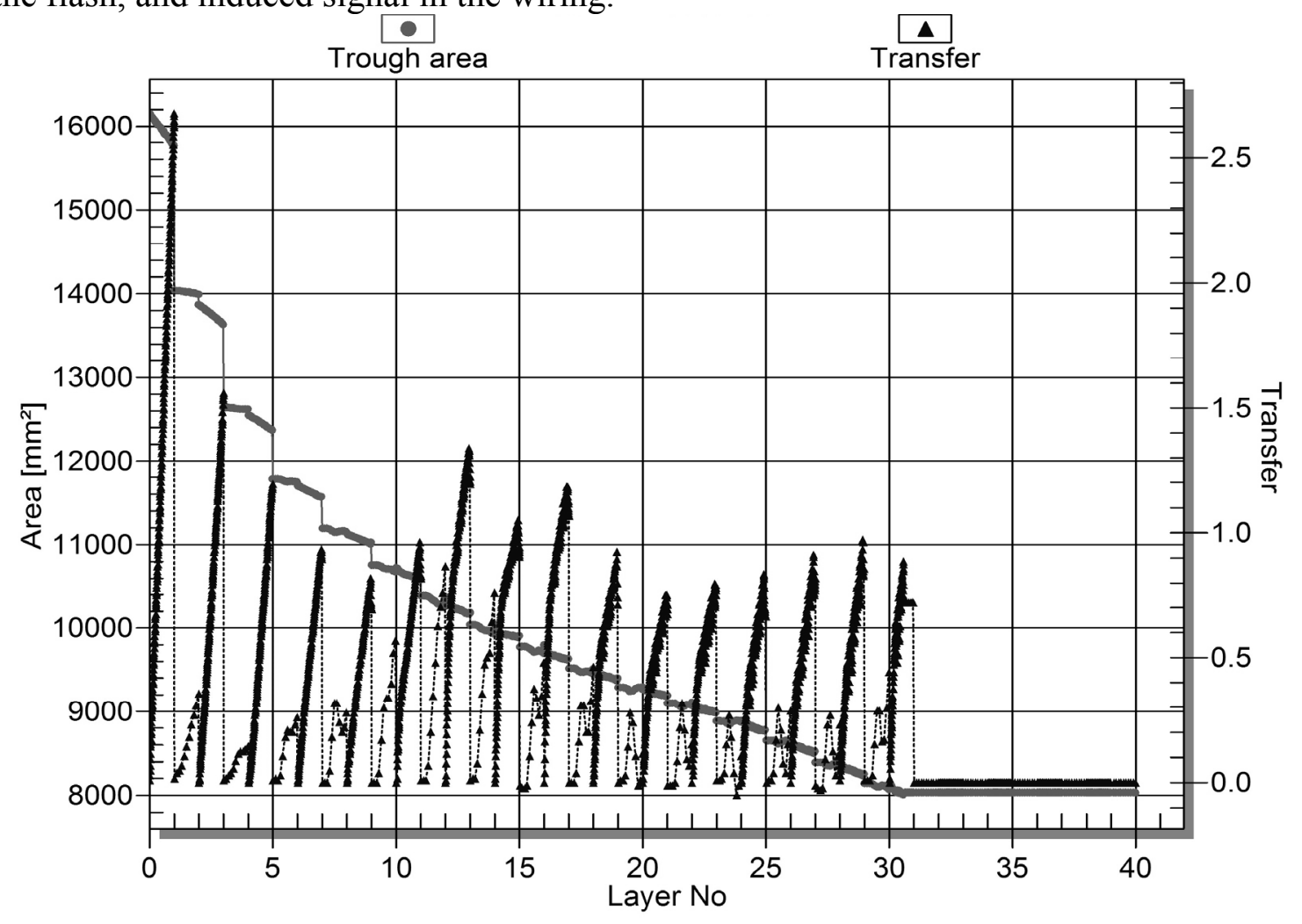

Figure 49. Trough area and transfer versus layer number. 


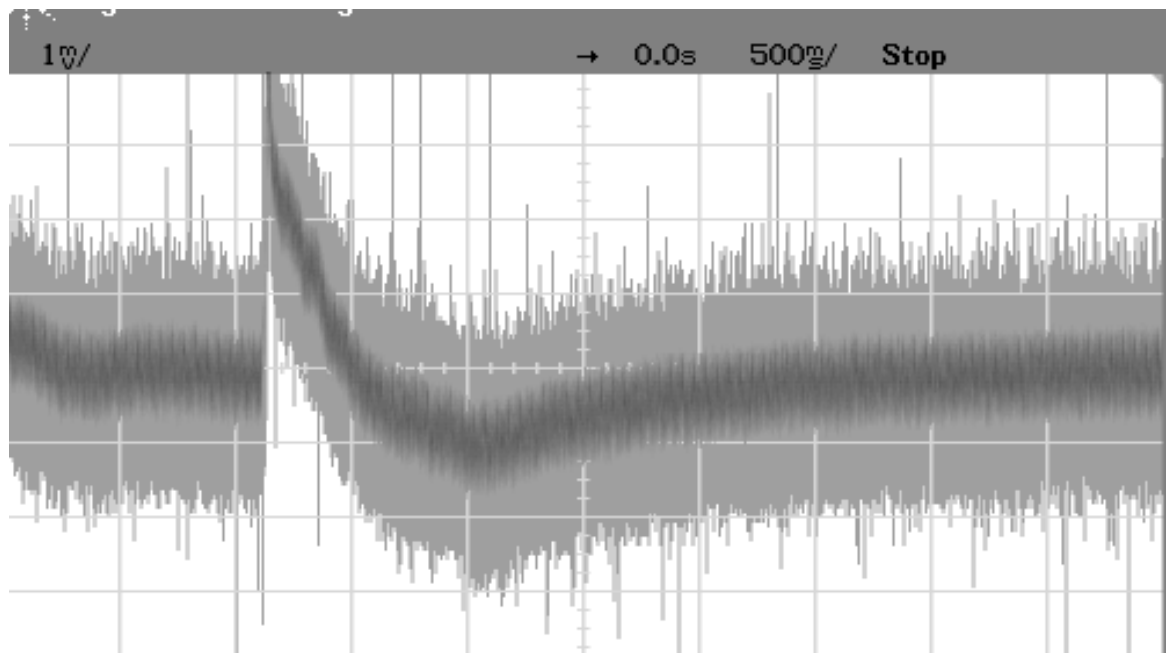

Figure 50. Photoelectrical response of 15 layers on an ITO substrate with a $5 \mathrm{~nm}$ thick gold top electrode measured with a digital oscilloscope.

Lifot $\mathrm{On}_{\mathrm{n}}$

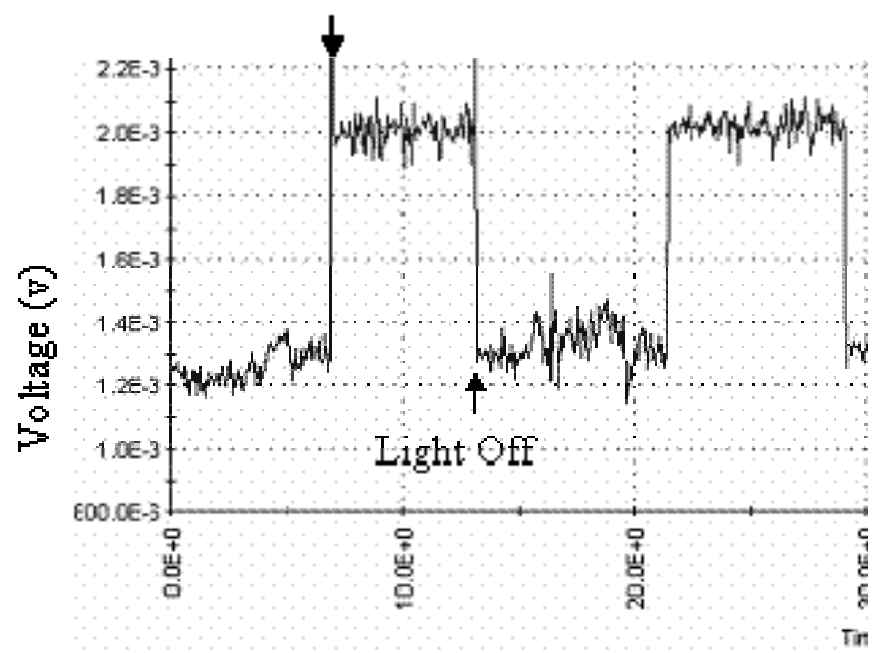

Figure 51. Photoelectrical response of 15 layers on an ITO substrate with a $5 \mathrm{~nm}$ thick gold top electrode measured with the Keithley 4200 SPA. 


\section{Chapter 6 Electronic Properties of Dried Films- Bacteriorhodopsin and Empirical Model}

To effectively integrate bacteriorhodopsin with microelectromechanical systems (MEMS) and nanoelectromechanical systems (NEMS) devices, it is critical to know the electrical properties of the material and to understand how it may affect the functionality of the device. Inductance, capacitance and resistance (LCR) measurements can be used to determine material characteristics, such as permittivity, film thickness, and capacitance. There has been little work conducted on measuring the electronic properties of bR films [78-81] and most have been done in the aqueous state. This section presents the LCR measurements of dried films of oriented and unoriented bR with both light-on and light-off conditions. Tests were performed on dried films of bR to determine if there is a relationship between LCR measurements and orientation, light-on/off, frequency, and time. The results indicated that the LCR measurements depended on the thickness and area of the film, but not on the orientation, as with other biological materials such as muscle. However, there was a transient LCR response for both oriented and unoriented bR which depends on light intensity.

Samples were prepared with different thicknesses. The stock solution $\left(6.9 \mathrm{mg} \mathrm{mL}^{-1}\right)$ was diluted with deionized distilled water. The bR was deposited over a uniform area $(9.62 \mathrm{x}$ $10^{-6} \mathrm{~m}^{2}$ ) on ITO coated glass using a method described in [54]. $20 \mu \mathrm{L}$ samples were oriented using electrophoretic deposition at $4 \mathrm{~V}$ for 40 seconds across a $1 \mathrm{~mm}$ gap. Unoriented samples were prepared by pipetting $20 \mu \mathrm{L}$ onto ITO glass. The samples were then dried for two days at $21{ }^{\circ} \mathrm{C}$. The voltage was measured using the operational amplifier circuit setup described earlier. The samples were photon activated using amber light emitting diodes as shown in Figure 38. The thickness of each sample was measured using a VEECO Dektak 6M Stylus Profiler. The average thicknesses of the samples were estimated from the data obtained. Absorbance measurements of the dried samples were made using an Ocean Optics USB4000 spectrometer.

\subsection{Impedance Measurements and Suggested Empirical Model}

Impedance measurements were made with an HP 4284A LCR meter controlled through an interface module in a Keithley 4200 semiconductor parametric analyzer. The environmental noise, such as RF, was reduced by placing the test fixture for sandwiching the $\mathrm{bR}$ between ITO conductors in a grounded enclosure. Again to reduce RF noise, bulkhead BNC connectors were used to connect the sample with the four terminals of the LCR meter. To minimize sources of error such as parasitic capacitance in the LCR 
measurements, open and short correction measurements were performed prior to testing. For each recorded measurement a long integration time was utilized along with averaging of five consecutive measurements. Since the material is biological, the measurements were conducted with a relatively low-level signal of $10 \mathrm{mV}$ to minimize degradation of the bR during the measurement. The LCR measurements were recorded as a function of light intensity, frequency, voltage, and time. The LED light intensity at $570 \mathrm{~nm}$ was set at five discrete values $\left(0.0,27.1,40.9,134.0\right.$, and $196.1 \mathrm{~W} / \mathrm{m}^{2}$ at $\left.570 \mathrm{~nm}\right)$, frequency was swept from $20 \mathrm{~Hz}$ to $1 \mathrm{MHz}$, and voltage was swept from $+/-2 \mathrm{~V}$ in $10 \mathrm{mV}$ increments.

The impedance measurements versus time and light intensity are shown in Figure 52 Figure 55. The oriented and unoriented samples show similar trends in both the capacitance $\left(C_{p}\right)$ and resistance $\left(R_{p}\right)$ versus time and light intensity. Figure 52 and Figure 53 display transient increases in $\mathrm{C}_{\mathrm{p}}$ with light activation and light intensity. This was probably due to the change in the charge density at the surface of the ITO electrodes, which was caused by the shifting of the protons in the bR. Figure 54 and Figure 55 indicate there was a decrease in $\mathrm{R}_{\mathrm{p}}$ with light activation which decreases with increasing light intensity. The data also suggests that the light affects oriented bR samples more than unoriented (Figure 55). Then, by plotting the change in resistance and capacitance versus light intensity at $570 \mathrm{~nm}$, as shown in Figure 56, a linear equation can be fit to the data which can be used to estimate the percent change in resistance and capacitance for a given light intensity. Further analysis of the resistance and capacitance data versus time and at different light intensity (Figure 57) indicates that the calculated RC time constant decreases with increasing light intensity.

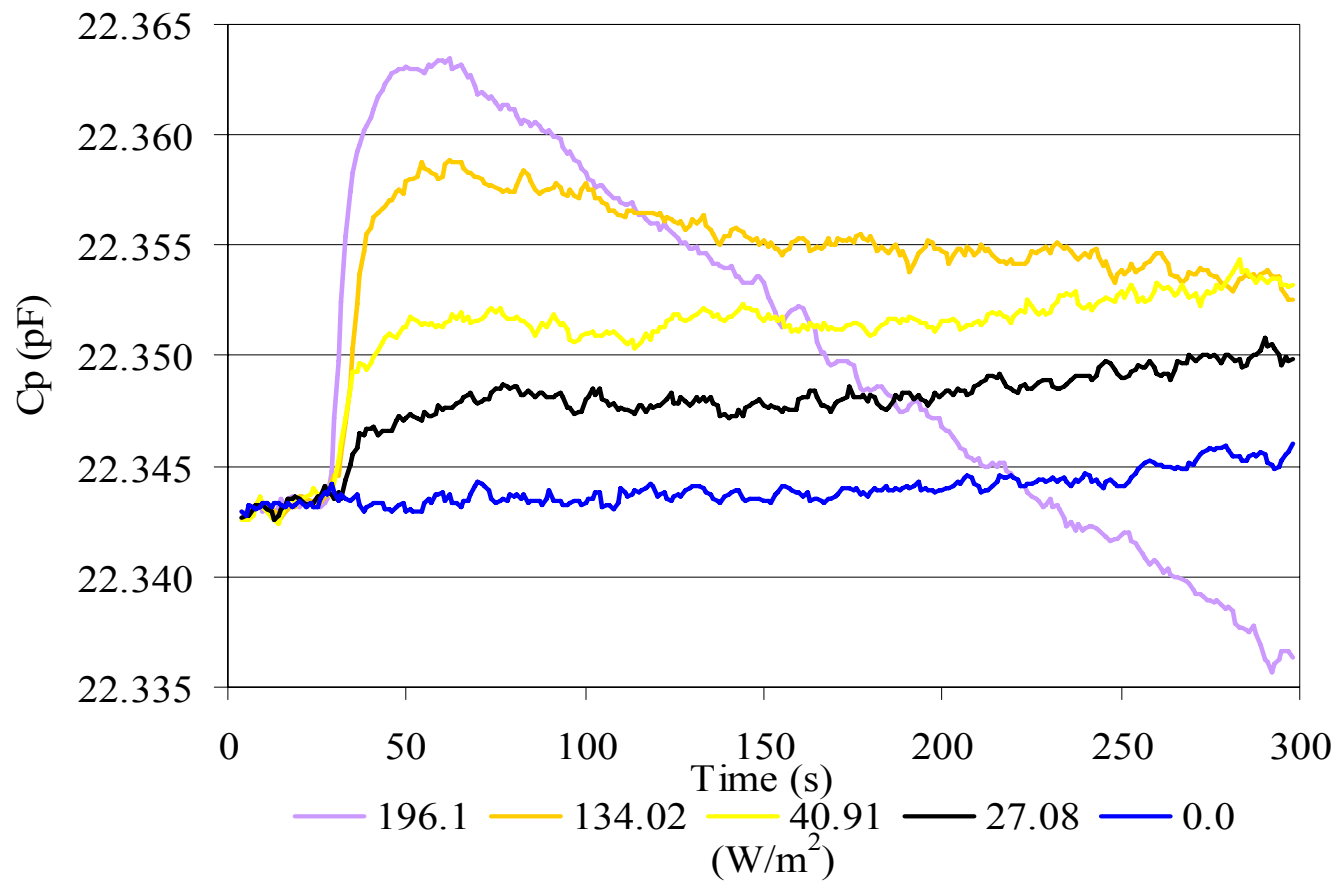

Figure 52. Parallel capacitance of dried bR versus time at four light intensities measured at $570 \mathrm{~nm}$ for the $10 \mu \mathrm{m}$ thick oriented sample (centered at light intensity of $0.0 \mathrm{~W} / \mathrm{m}^{2}$ ). 


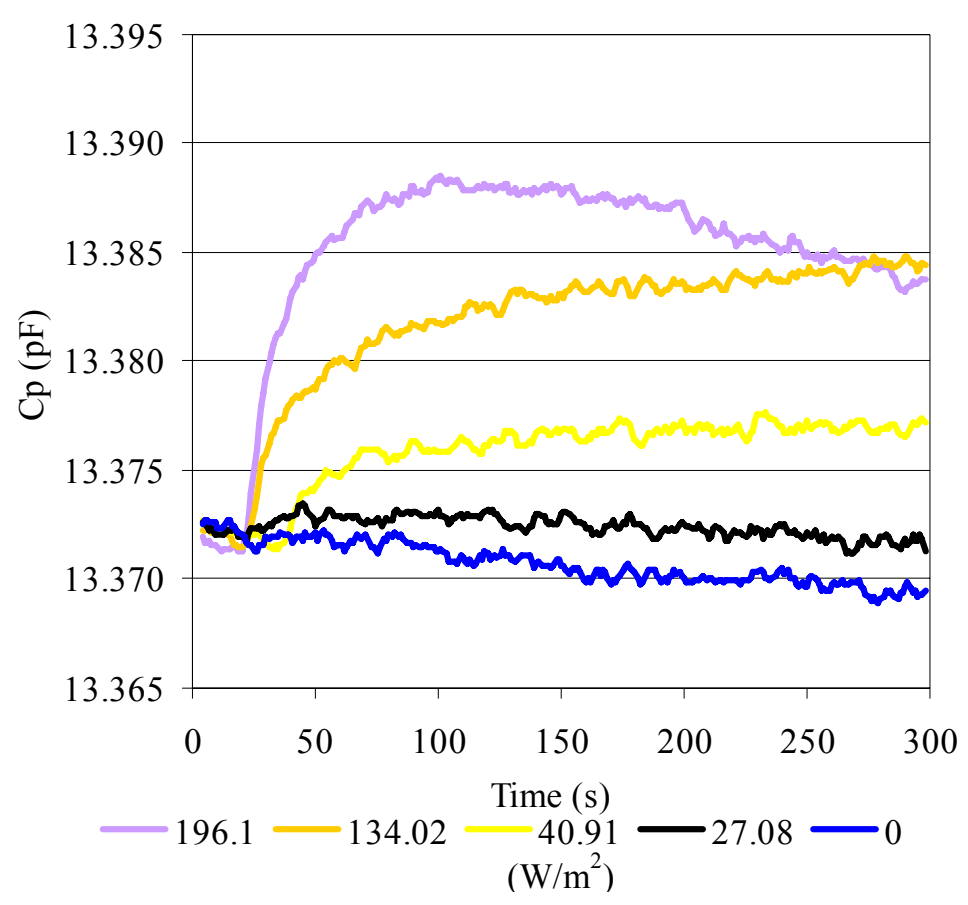

Figure 53. Parallel capacitance of dried bR versus time at four light intensities measured at $570 \mathrm{~nm}$ for the $22 \mu \mathrm{m}$ thick unoriented sample (centered at light intensity of $0.0 \mathrm{~W} / \mathrm{m}^{2}$ ).

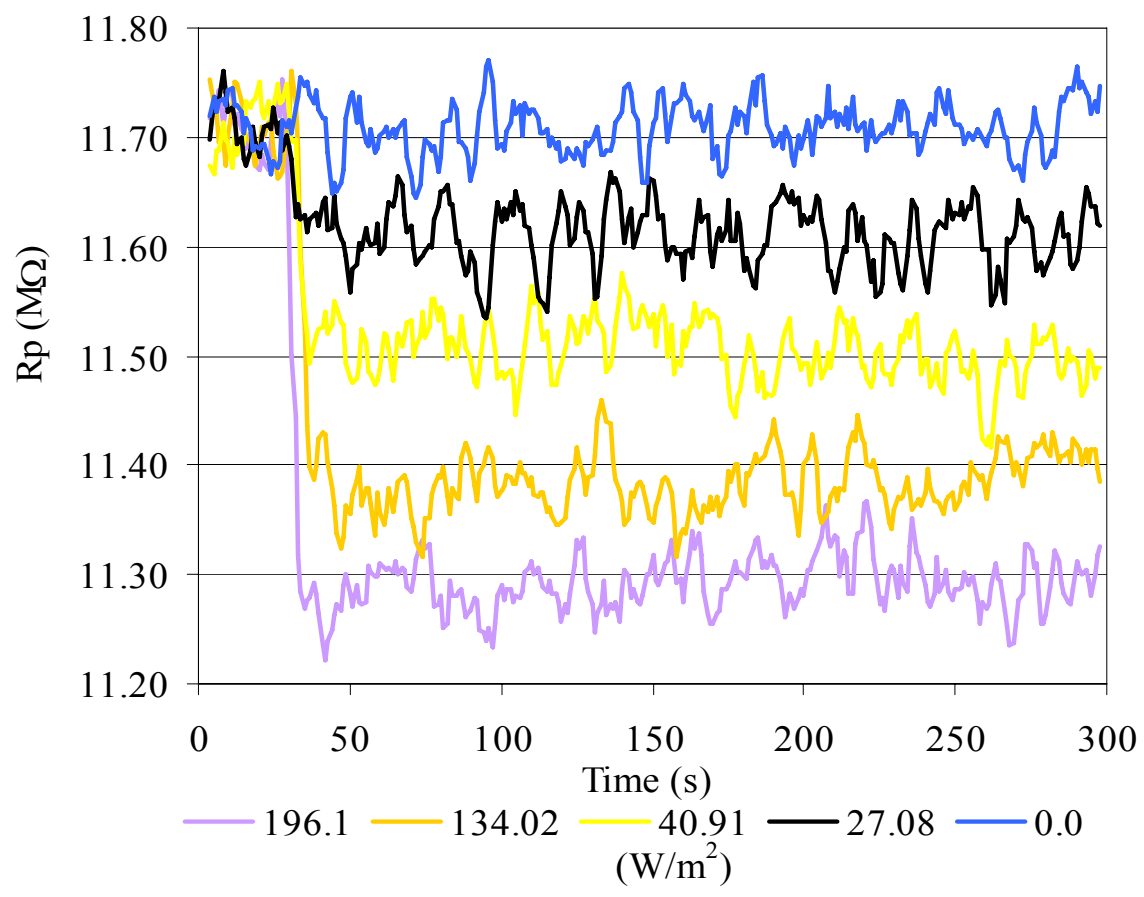

Figure 54. Parallel resistance of dried bR versus time at four light intensities measured at $570 \mathrm{~nm}$ for the $10 \mu \mathrm{m}$ thick oriented sample (centered at light intensity of $0.0 \mathrm{~W} / \mathrm{m}^{2}$ ). 


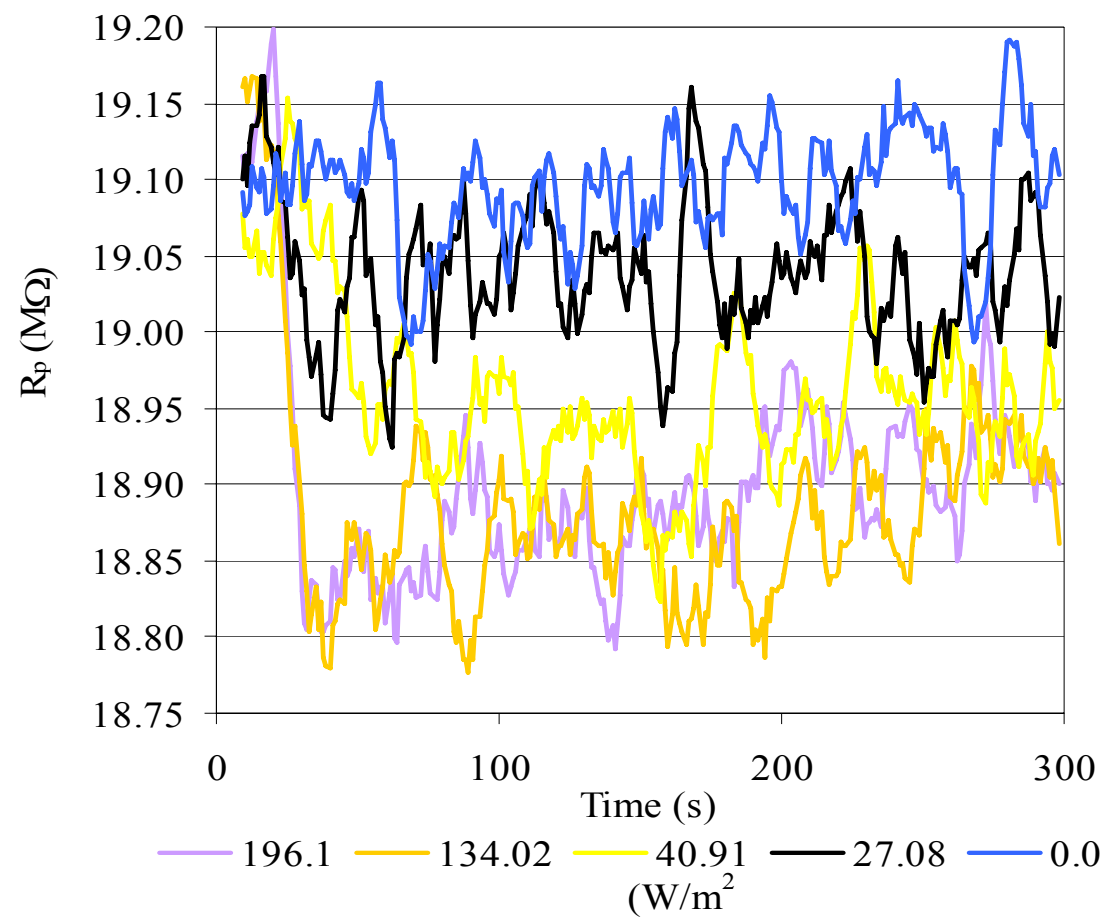

Figure 55. Parallel resistance of dried bR versus time at four light intensities measured at $570 \mathrm{~nm}$ for the $22 \mu \mathrm{m}$ thick unoriented sample (centered at light intensity of $0.0 \mathrm{~W} / \mathrm{m}^{2}$ ).

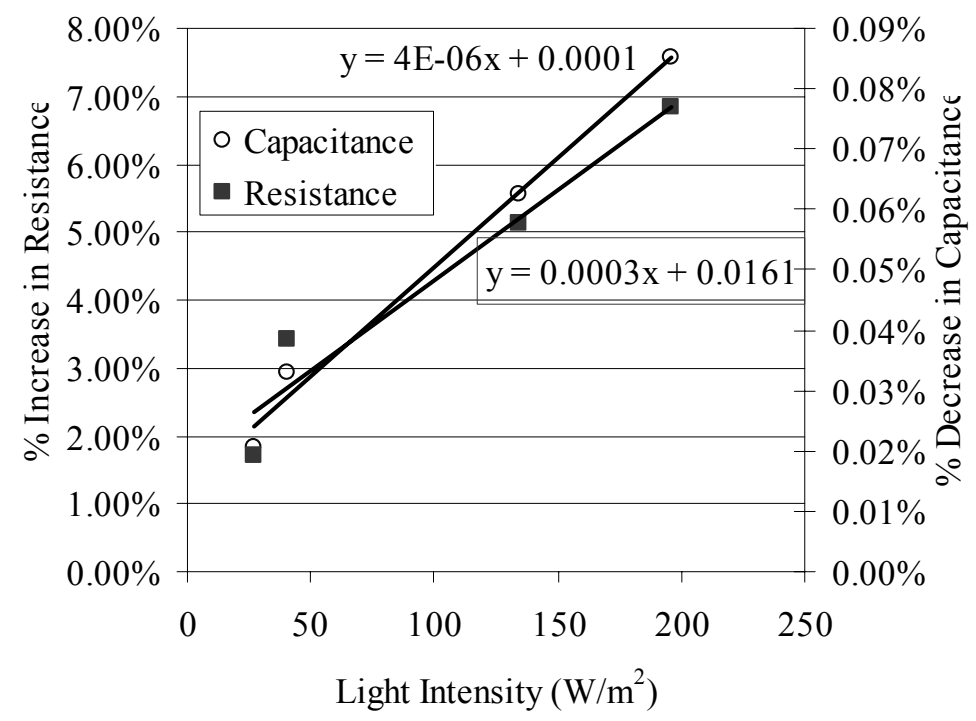

Figure 56. Change in resistance and capacitance versus light intensity measured at 570 $\mathrm{nm}$. 


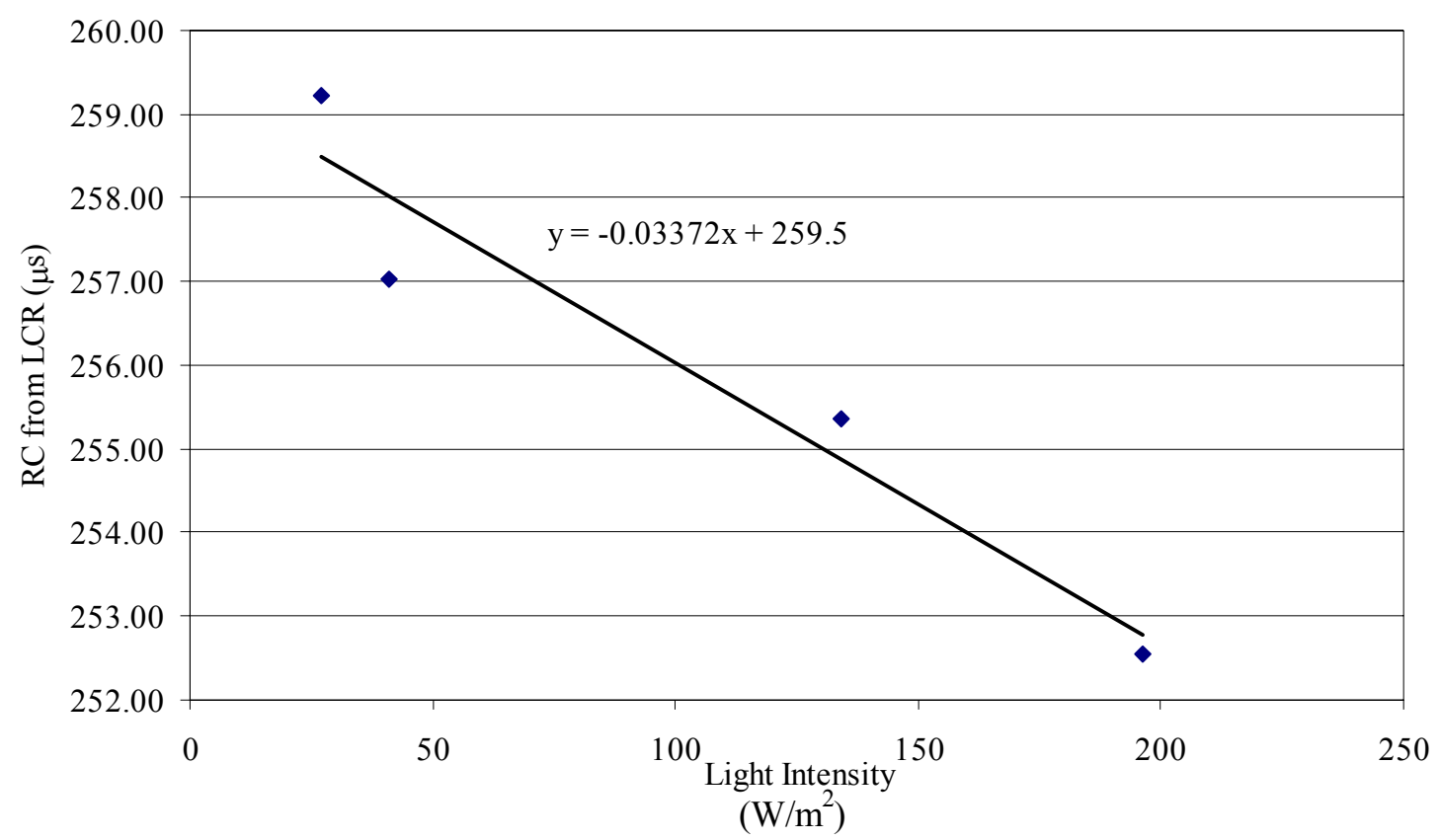

Figure 57. RC time constant calculation based on LCR measurements.

Frequency sweep measurements from $20 \mathrm{~Hz}$ to $1 \mathrm{MHz}$ in Figure 58 , indicates that the resistance and capacitance change slightly with frequency and shows no resonance points within the range tested. Knowing the area $\left(9.62 \times 10^{-6} \mathrm{~m}^{2}\right)$, thickness $(11 \mu \mathrm{m})$, and resistance as shown in Figure 58 of the dried bR film at a frequency $>1000 \mathrm{kHz}$, the resistivity ( $\rho$ ) is calculated to be $\sim 2 \mathrm{G} \Omega \mathrm{m}$, which is comparable to what others have calculated or estimated $[22,79] \rho$ numbers. The voltage sweep measurements (Figure 59) indicate that the capacitance does not change with applied voltage and also shows that the capacitance changes with thickness. As expected the overall capacitance increased with decreasing thickness as shown in Figure 59 and Figure 60.

Analyzing the capacitance versus thickness as shown in Figure 60 data revealed that the capacitance increases as the thickness decreases but the relationship appears to be linearly proportional instead of inversely proportional to the thickness, as expected for a parallel plate capacitance. This could be related to a number of variables such as the topology of sample, the variation in humidity within the sample, variation in the contact area between the ITO-PM-ITO interfaces for all the samples, the complex interaction and alignment between multi-monolayers of PM, and current tunneling or transport through the PM. These results indicate that $\mathrm{bR}$ is dielectric material but further research should be conducted to verify these results, possibly at the monolayer level. It is also important to note that there was no significant difference in the capacitance for oriented and unoriented if the area, thickness, and environmental conditions were constant, as they were for these experiments. From Figure 60 the relative permittivity was estimated at 2.2 which is in agreement with [82]. 


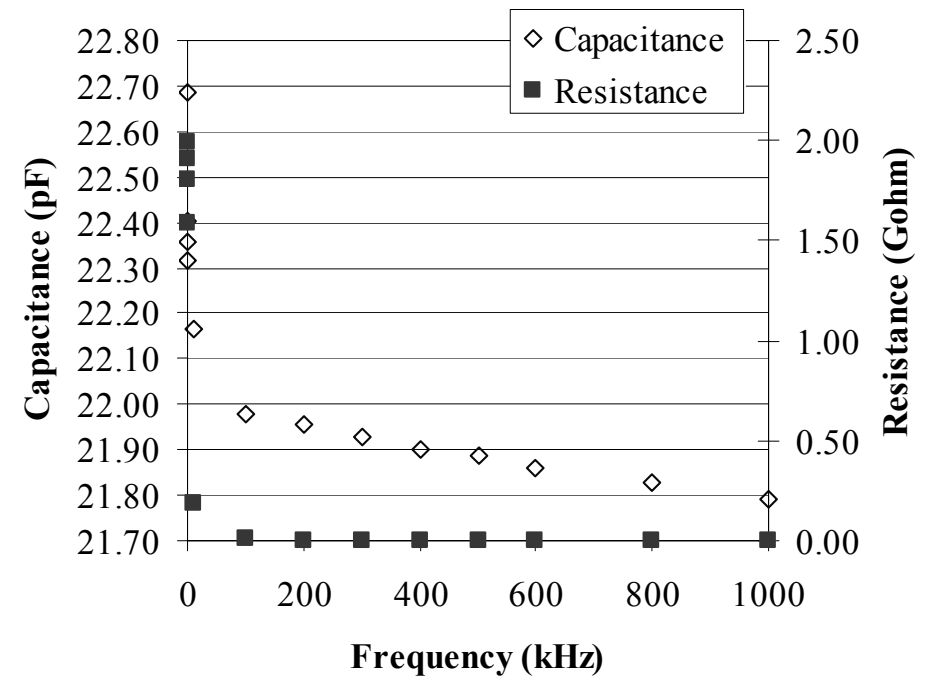

Figure 58. Capacitance and resistance of dried bR versus frequency.

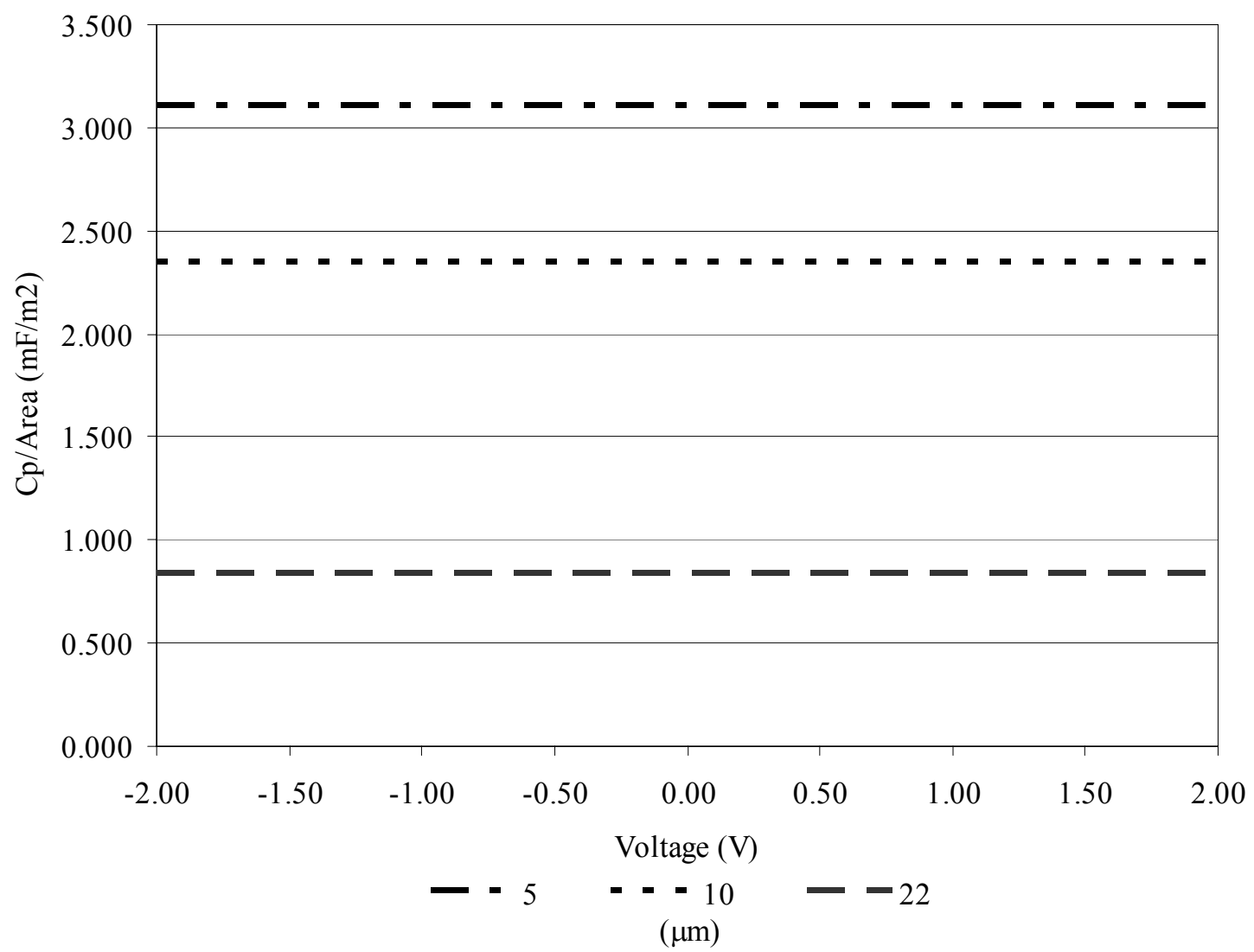

Figure 59. Capacitance per area versus voltage for $5 \mu \mathrm{m}$ and $10 \mu \mathrm{m}$ thick oriented samples and the $22 \mu \mathrm{m}$ thick unoriented sample. 


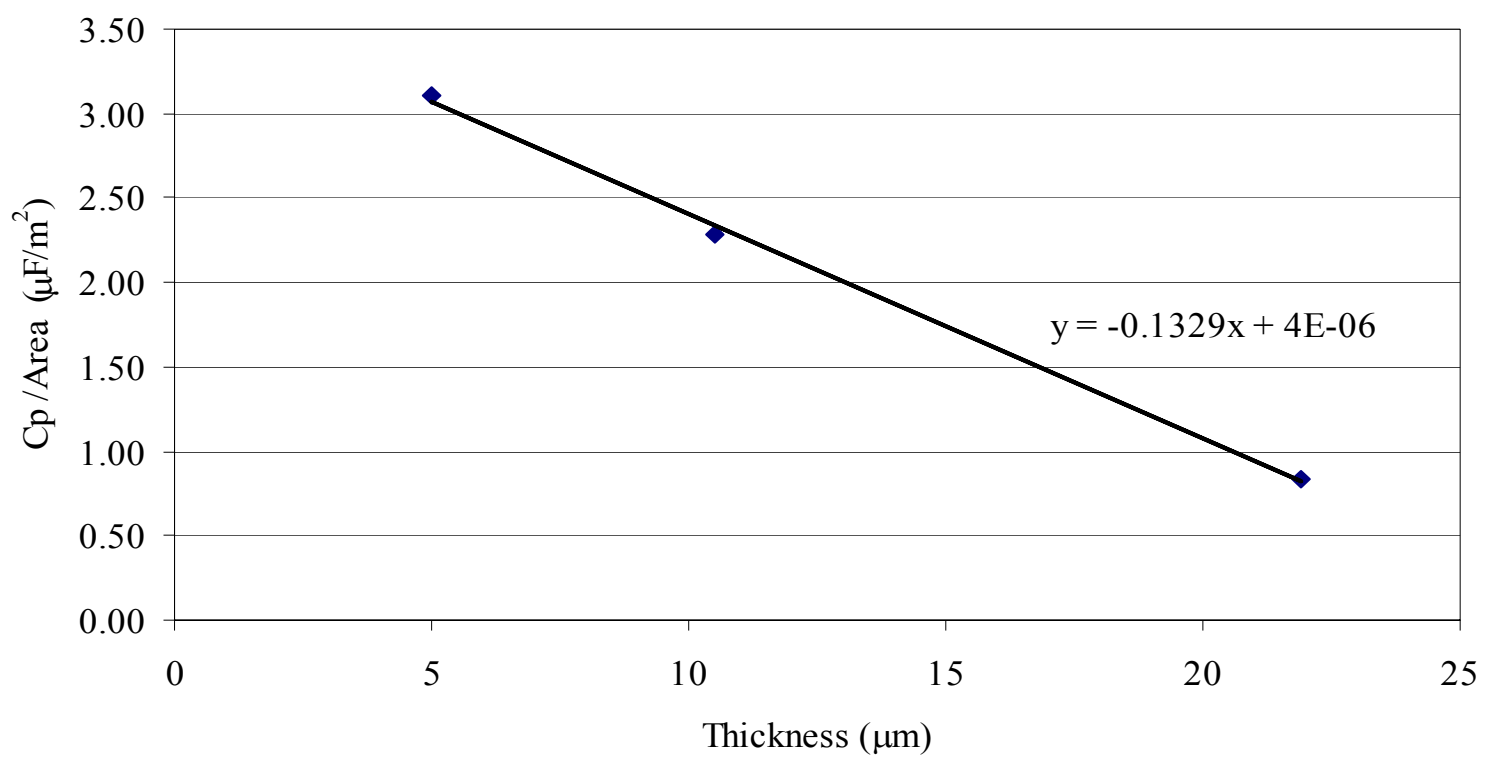

Figure 60. Capacitance per area versus average thickness for both oriented and unoriented samples.

The impedance measurements demonstrated that there was a change in capacitance and resistance upon illumination for both oriented and unoriented samples. These changes appear insignificant considering they were on the order of 5 to $20 \mathrm{fF}$, but when integrated with a SET with a total capacitance less than an aF, this change in capacitance could be significant and might alter the functionality of a SET. From the LCR measurements of the dried films, it was determined that the dominant electrical characteristics of the $\mathrm{bR}$ were the parallel capacitance $\left(C_{p}\right)$ and parallel resistance $\left(R_{p}\right)$ for light activated and not activated, and oriented and unoriented samples. Since the $R_{p}$ was greater than the series resistance $\left(R_{s}\right)$, and the parallel capacitance $C_{p}$ was small, this implies that dried films of bR can be modeled as a capacitor and resistor in parallel when placed between conductive electrodes as shown in Figure 61.

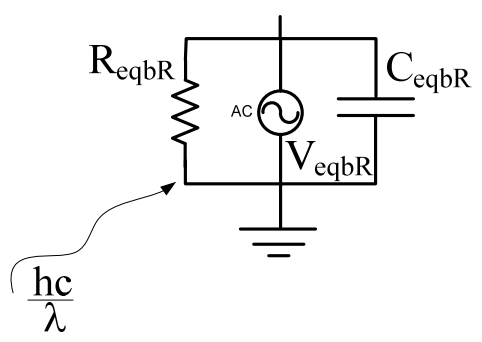

Figure 61. Representative schematic model of dried films of $b R$, where $R_{\text {eqbR }}$ is the equivalent resistance of the dried $\mathrm{bR}$ film, $\mathrm{C}_{\text {eqbR }}$ is the equivalent capacitance of the dried $\mathrm{bR}$ film, $\mathrm{h}$ is Planck's constant $\left(\mathrm{h}=6.626 \times 10^{-34} \mathrm{~J} \mathrm{~s}\right), \mathrm{c}$ is speed of light $\left(\mathrm{c}=3 \times 10^{8} \mathrm{~m} / \mathrm{s}\right)$, and $\lambda$ is wavelength in nanometers. 


\subsection{Operational Amplifier Circuit Measurements and Empirical Model}

\subsubsection{Light Activated RC Time Constant Based on Photovoltaic Response}

The photovoltaic response was analyzed based on the activation light intensity in the same test fixture used for the LCR measurements and with the same light intensities at $570 \mathrm{~nm}$. The light intensity was controlled by the supply current to the LEDs by fixing the distance between the sample and the LEDs and exposed area. As shown, in Figure 62 , the thickest oriented sample produced the largest voltage. While the smallest photovoltaic response produced by an unoriented sample (thickness approximately 22 um) was about $25 \mathrm{mV}$ at all light powers, negligible in comparison to oriented samples. The response of the oriented samples was approximately $100 \mathrm{X}$ greater than the unoriented. The voltage gradient generated by the oriented bR was much greater than the unoriented $b R$ due to the vectorial nature of the proton shifting within the $b R$. When $b R$ was electrophoretically deposited, it orients with the intracellular side down and either attaches to the electrode or stacks on the previous layer. The voltage output is the sum of the voltage produced by each monolayer. In unoriented samples the layers of the extracellular and intracellular sides of the bR are randomly oriented, causing charge cancellation and therefore a small voltage output.

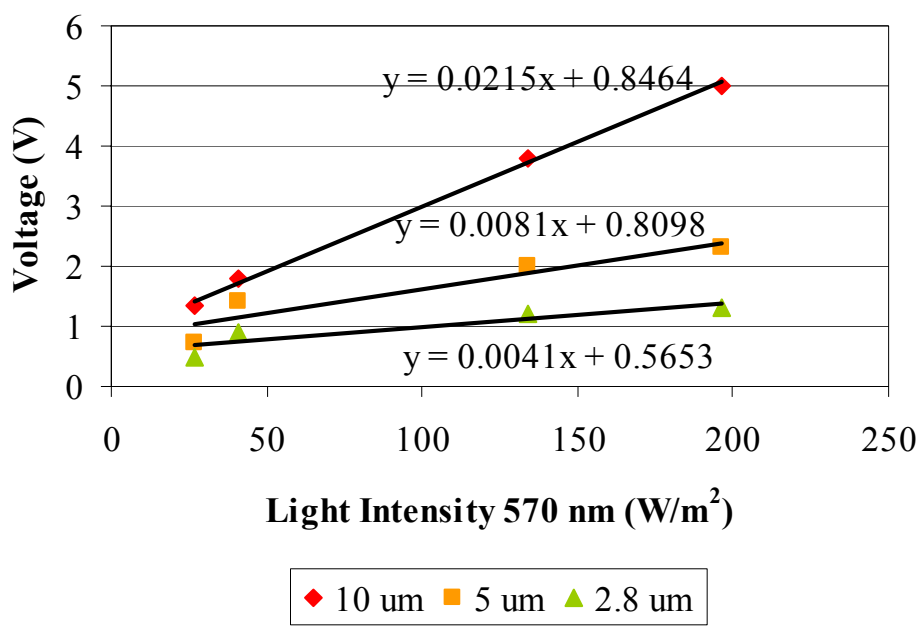

Figure 62. Photovoltaic output of 3 samples with thickness of 2.8, 5̣and $10 \mu \mathrm{m}$ at 4 light intensities measured at $570 \mathrm{~nm}$.

Figure 62 shows that the magnitude of the photovoltaic response of bR increases linearly with increasing light intensity and the thickness of the sample. The data shown in Figure 62 was determined by taking the difference in the maximum voltage and minimum voltage for a particular light intensity from data presented in Figure 63 - Figure 65. 
Fitting the transient photovoltaic curves shown in Figure 63 - Figure 65, to the model suggested in Figure 61, can be represented mathematically by Eq. (4) shown below;

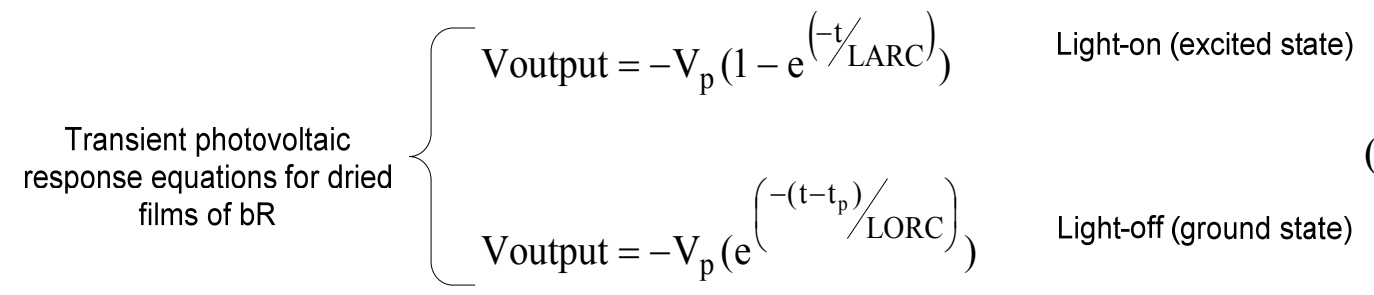

where $V_{p}$ is the peak voltage, $t_{p}$ the time the light in turned-off, LARC is the light activated time constant, and LORC light-off time constant. The light activated time constant (LARC) is defined as the time it takes excited bR to reach $63.2 \%$ of maximum photovoltaic output measured in DC mode (i.e. not capacitively coupled). $V_{p}$ can be estimated from the equations shown in Figure 62. Figure 63 - Figure 65 show that the empirical model of the transient photovoltaic response fit the experimental data well.

In much the same way that voltage response is based on the thickness of the sample and light intensity as shown earlier, the (LARC) time constant is dependent on these two factors as shown in Figure 66. This figure indicates that the LARC decreases with increased light intensity and decreased sample thickness, which is in good agreement with published photovoltage signals of dried monolayers of bR [83] which have almost instant activations. As previously suggested by the LCR measurements, these results also suggest that either the resistance or capacitance, or both, are changing with light intensity. The LORC is significantly different from the LARC just as other have presented $[17,83$, 84], these differences may be associated with a difference in conductivity within the bR when excited (light-on) and not excited (light-off) as shown [78] and with I-V curves of monolayers with both light activated and dark adapted. For all curves in Figure 63 Figure 65 the LORC time constants appear to be similar in shape and rate of decrease.

Comparing the LARC, Figure 66 and the RC time constants (Figure 57) measured with the LCR meter, show that in general both time constants decreased with increased light intensity. But the relative magnitude of LARC and RC time constants are significantly different, which is probably do to the fact that they were estimated by two different measurement methods which had different input impedances and include the characteristics of measurement instrumentation. 


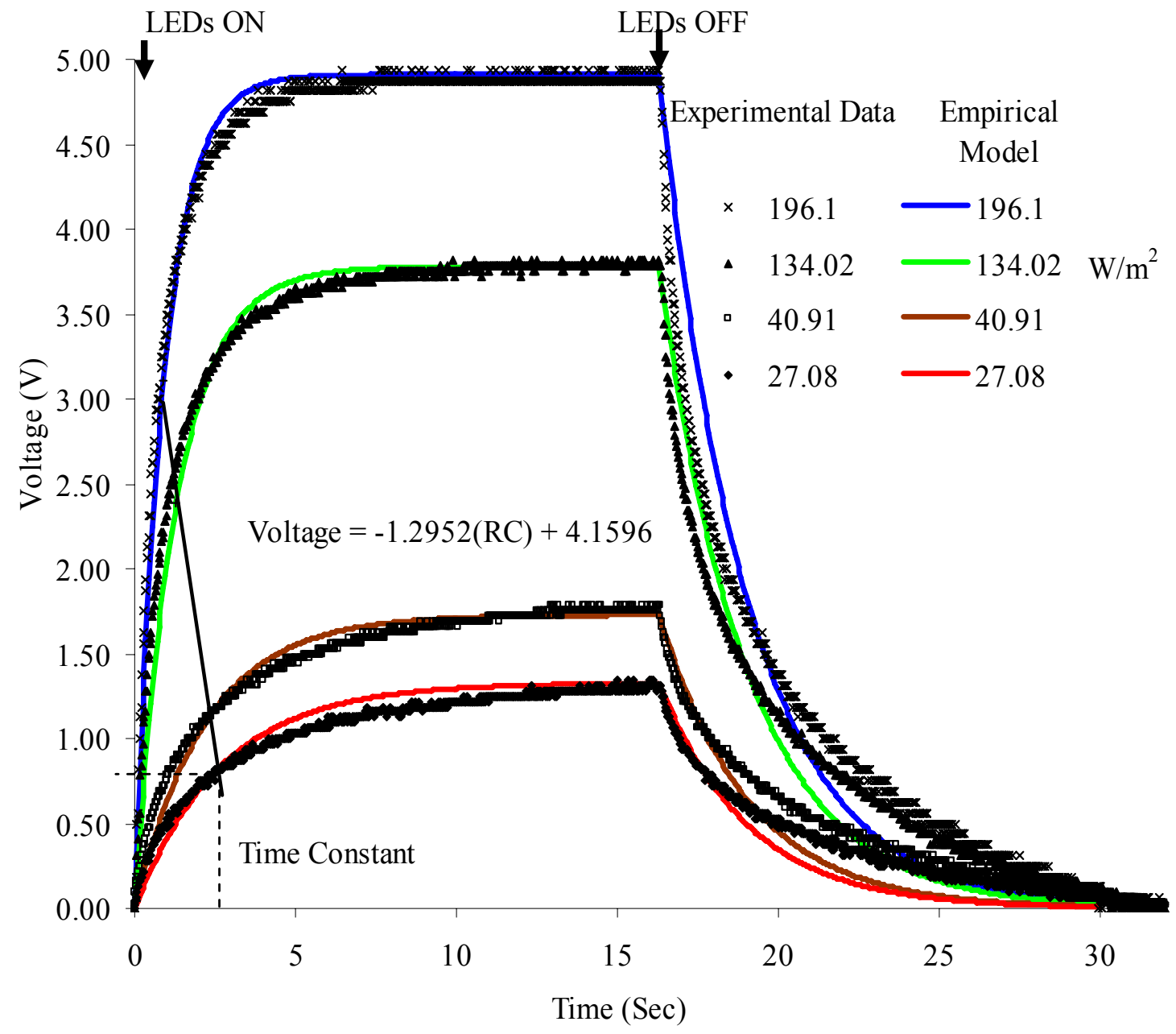

Figure 63. Photovoltaic responses of a $10 \mu \mathrm{m}$ thick sample at four different light intensities measured at $570 \mathrm{~nm}$. 


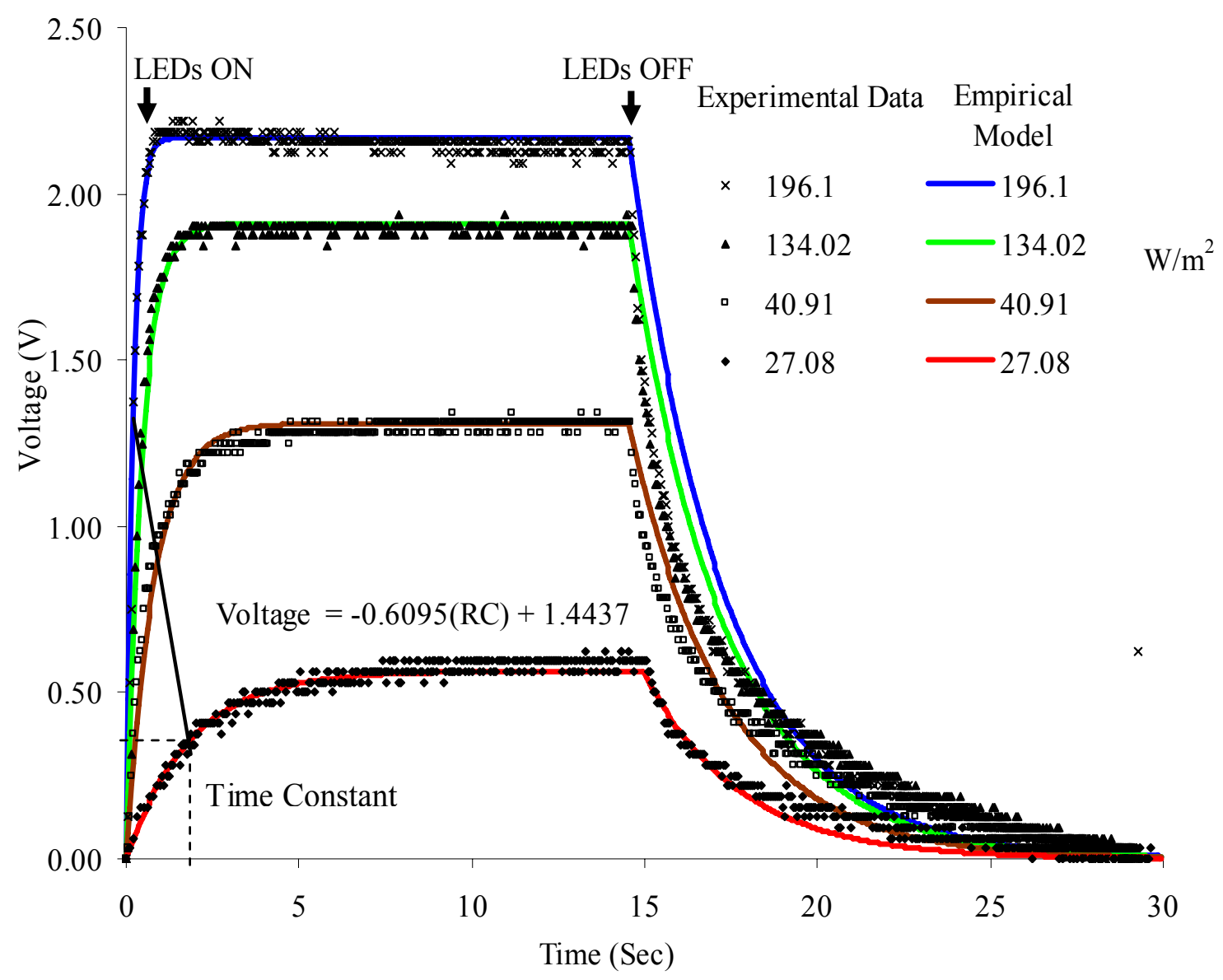

Figure 64. Photovoltaic responses of a $5 \mu \mathrm{m}$ thick sample at four different light intensities measured at $570 \mathrm{~nm}$. 


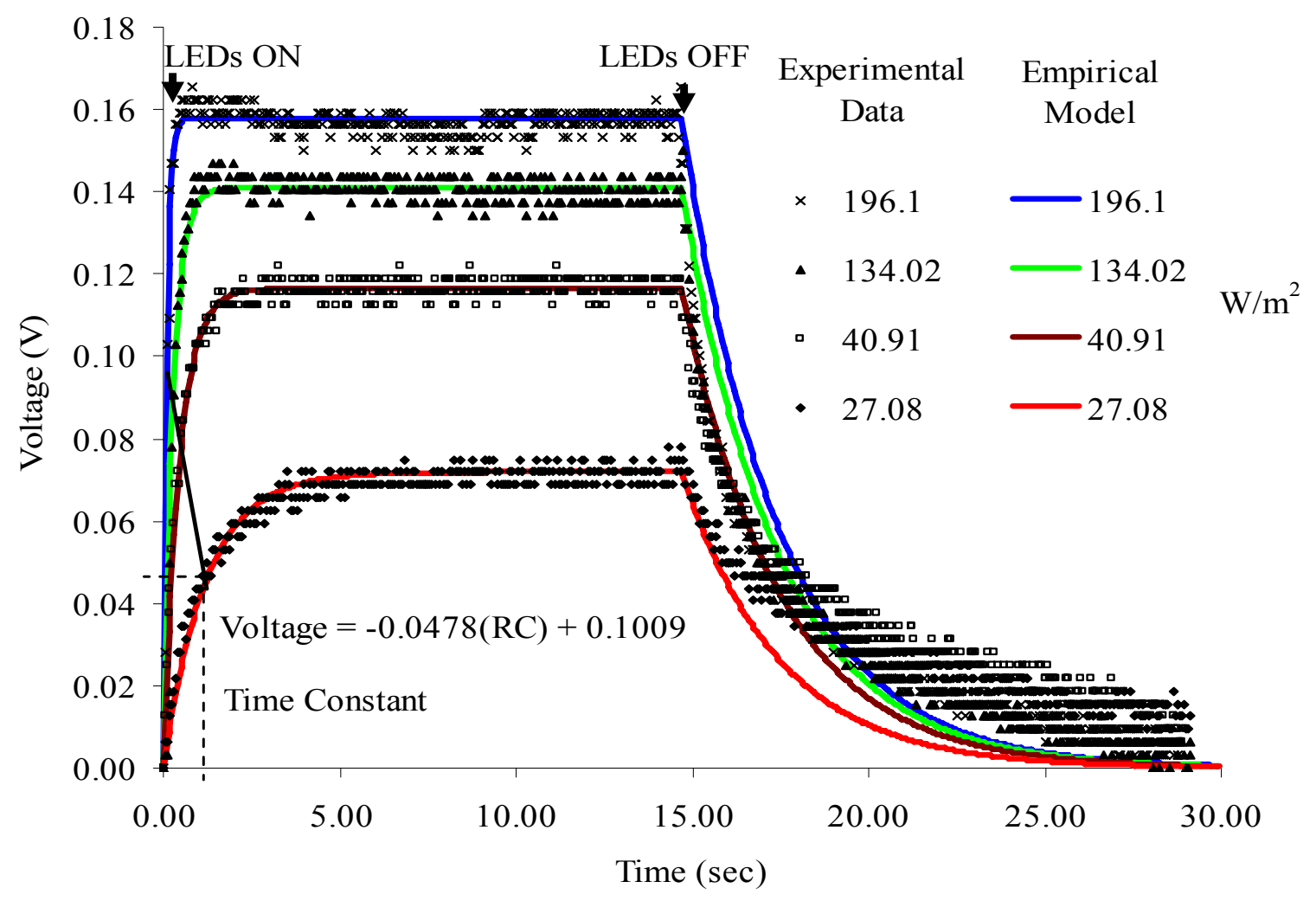

Figure 65. Photovoltaic responses of a $2.8 \mu \mathrm{m}$ thick sample at four different light intensities measured at $570 \mathrm{~nm}$.

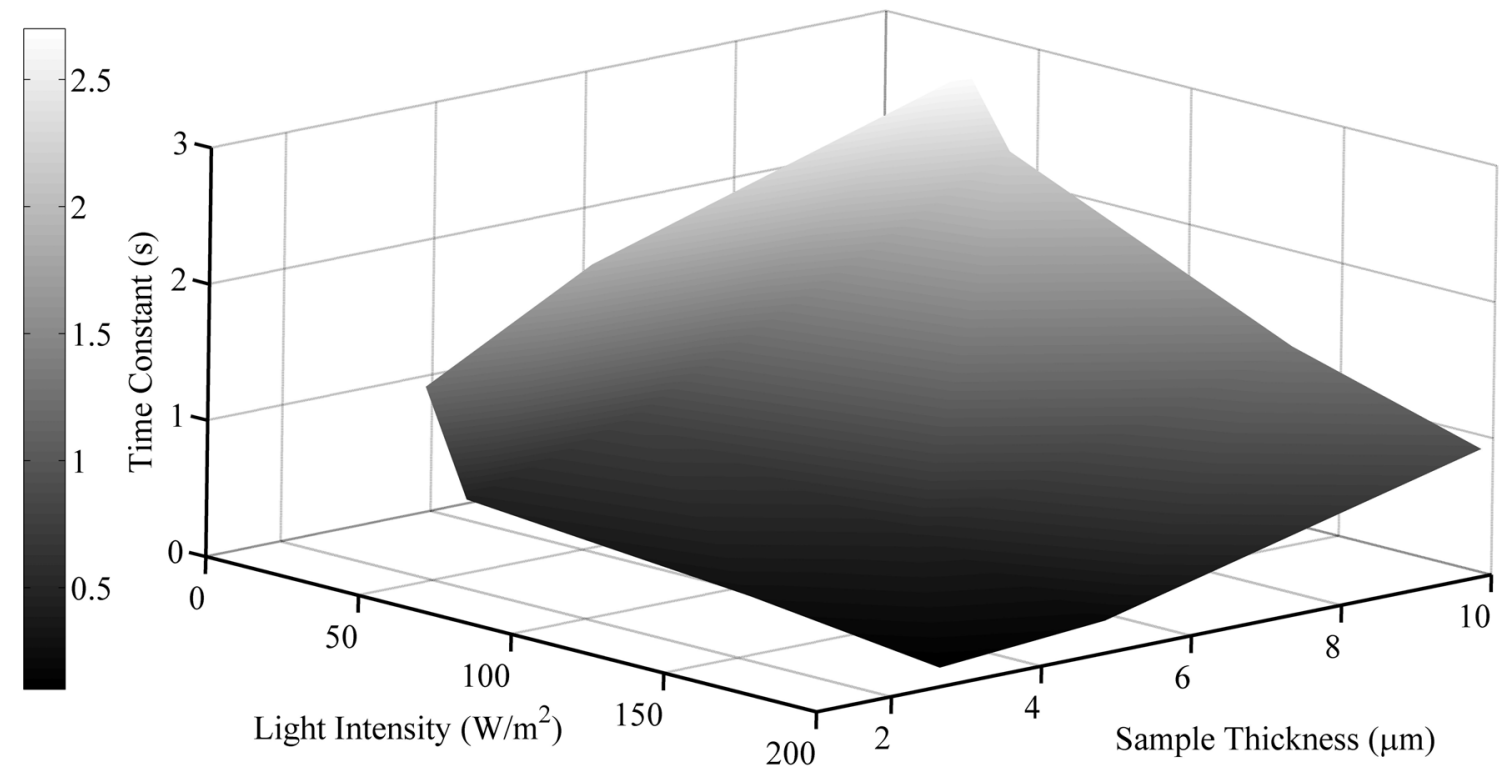

Figure 66. Surface plot of the light activated time constant of bR with respect to light intensity at $570 \mathrm{~nm}$ and thickness of bR sample. 
To eliminate a possible contribution to the transient photovoltaic response, the LEDS and the op-amp circuit were examined. Through literature and experimental research it was determined that the LEDs have almost instantaneous switching speeds, nano to micro second regime, from on to off or off to on [85]. There is no flickering or warm up period as with conventional lighting [85]. The fast turn-on time of the LEDs confirms that the LEDs are not significantly contributing to LARC and LORC of the bR.

The op-amp circuit was also calibrated by inputting a square wave with peak-to-peak voltage in the range of $25 \mathrm{mV}-10 \mathrm{~V}$ regimens at a variety of frequencies from $0.1 \mathrm{~Hz}-$ $10 \mathrm{~Hz}$-formed by a function generator. Calibration consisted of measuring the gain and skew, by comparing the inputted square wave, from the function generator, to the output wave form from the op-amp circuit. From this it was determined that the voltage gain was 1 and that there was no noticeable difference in the input voltage from a function generator and output voltage the op-amp circuit for these low frequency measurements.

\subsubsection{Analogous to Capacitors in Series}

Bacteriorhodopsin photocells behave analogous to capacitors in parallel and series. In parallel, the two photocell photoresponses summed according to Eqs. (5), effectively doubling the overall activated area. When connected in series the total photovoltaic response was almost un-measurable. The photovoltaic response seemed reduced according to Eqs (5) - (6).

The bR photocells were arranged as shown in Figure 67 to measure the interaction between them in parallel and series.

$$
\begin{gathered}
\mathrm{C}_{\text {total }}=\mathrm{C}_{1}+\mathrm{C}_{2} \\
\frac{1}{\mathrm{C}_{\text {total }}}=\frac{1}{\mathrm{C}_{1}}+\frac{1}{\mathrm{C}_{2}}
\end{gathered}
$$



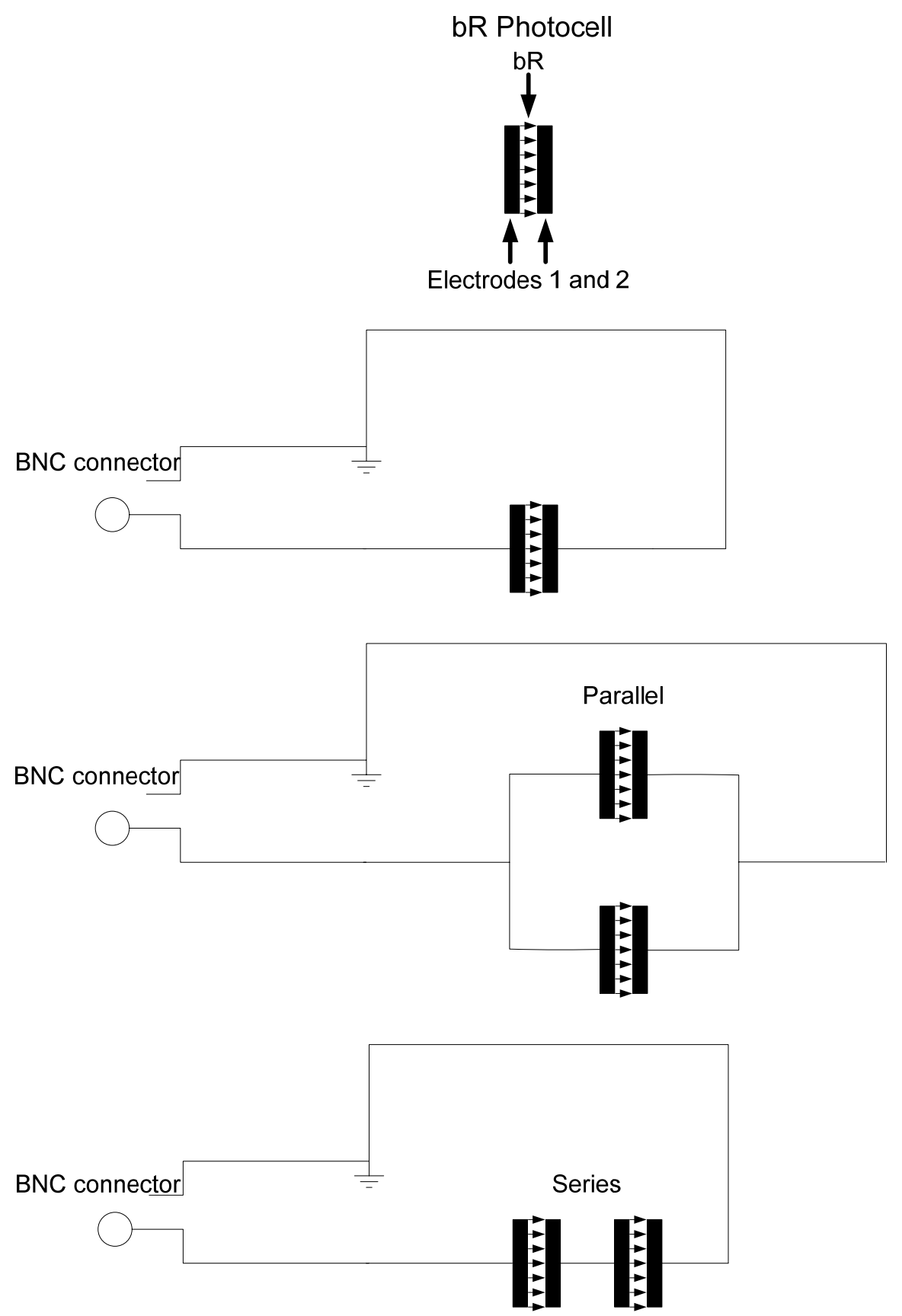

Figure 67. bR photocell layout.

The bR photocells were activated with laser pointers which had an output of 3-4 $\mathrm{mW}$ at $570 \mathrm{~nm}$. With two bR photocells in parallel and activated simultaneously, the output signal was doubled, as shown in Figure 68 and Figure 69. When the bR photocells were placed in series there was no measurable signal produced. These results are consistent with how capacitors act in parallel and series. 
Also studied was the activation of a single bR photocell with one laser and then two lasers, each with approximately the same power output, simultaneously and delayed, as shown in Figure 70 and Figure 71. These results will be helpful when designing the bR gate pad for the SET.

- Photocells 1\&2 - Photocell 1 - Photocell 2

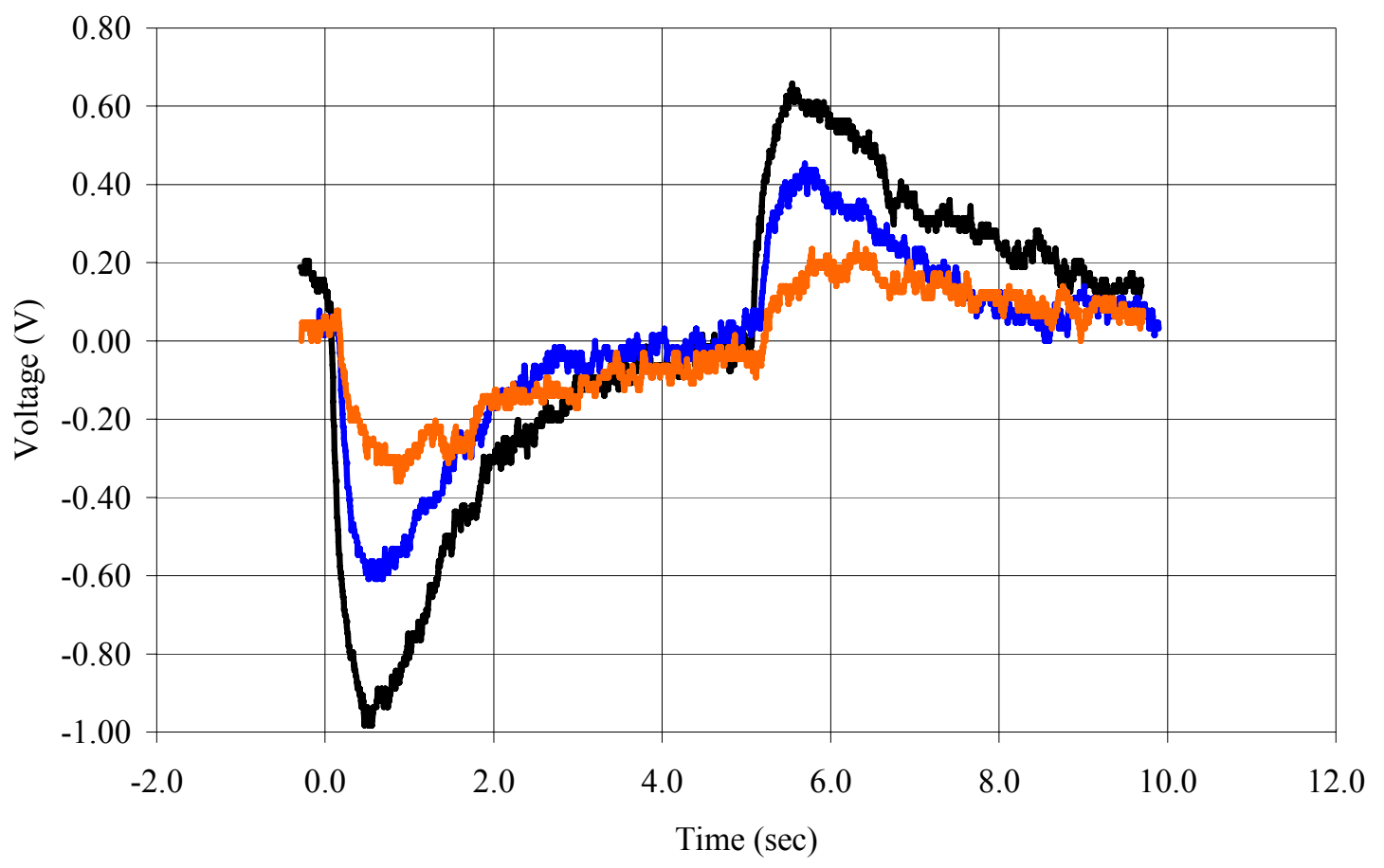

Figure 68. Unamplified bR photoresponse to a $0.1 \mathrm{~Hz}$ excitation. 


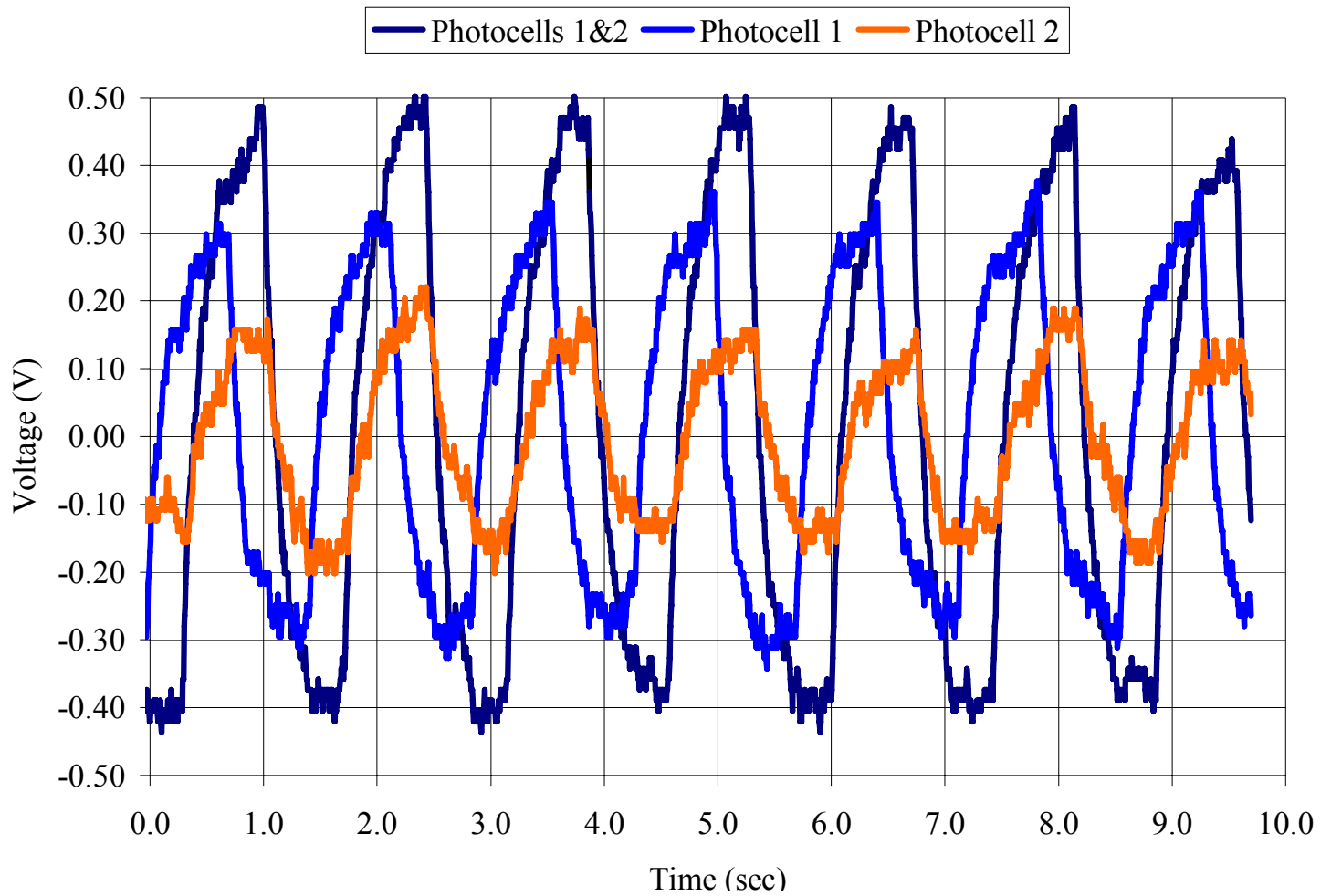

Figure 69. Unamplified bR photoresponse to a $0.7 \mathrm{~Hz}$ excitation.

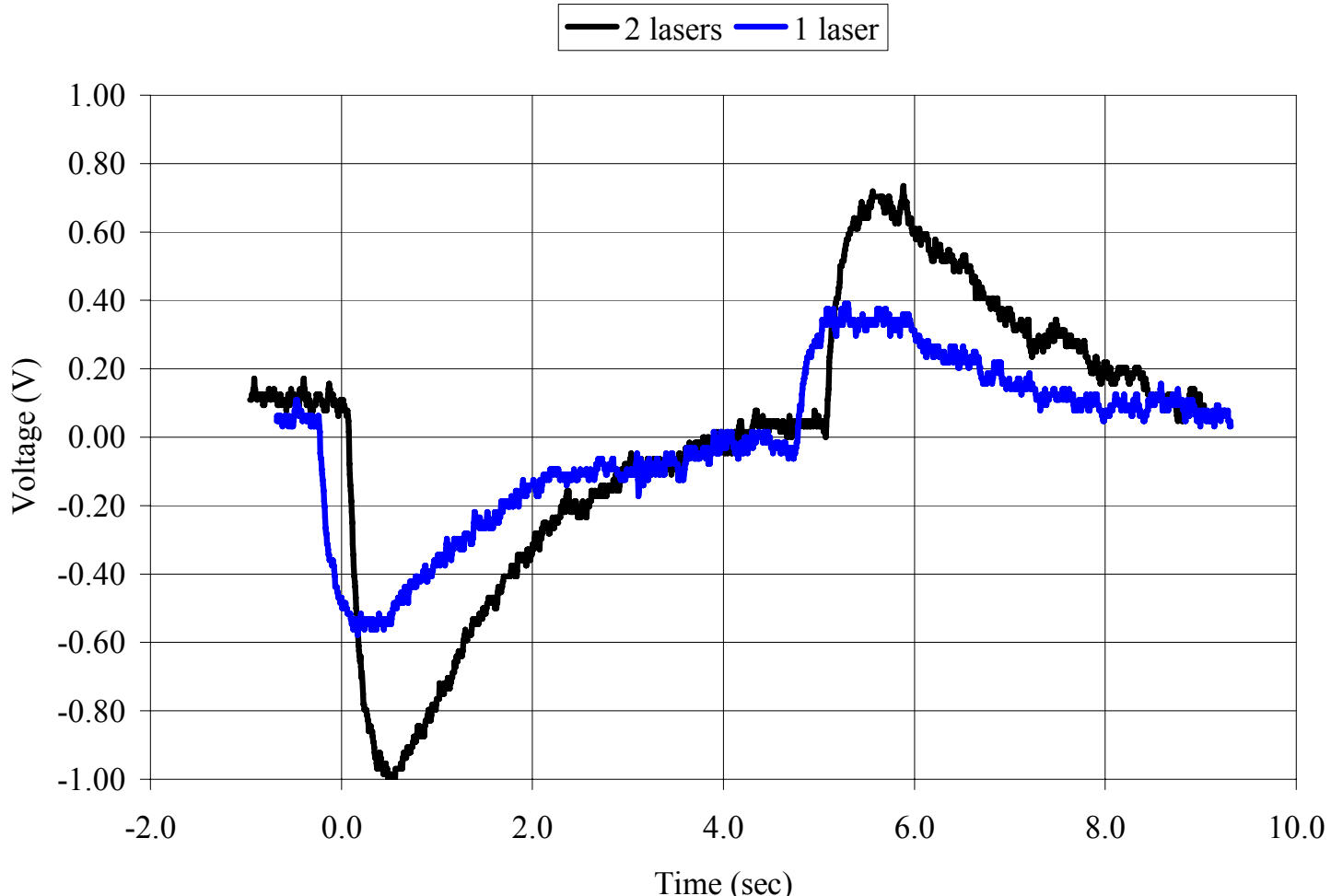

Figure 70. Unamplified bR photoresponse of single photocell with one and two lasers. 


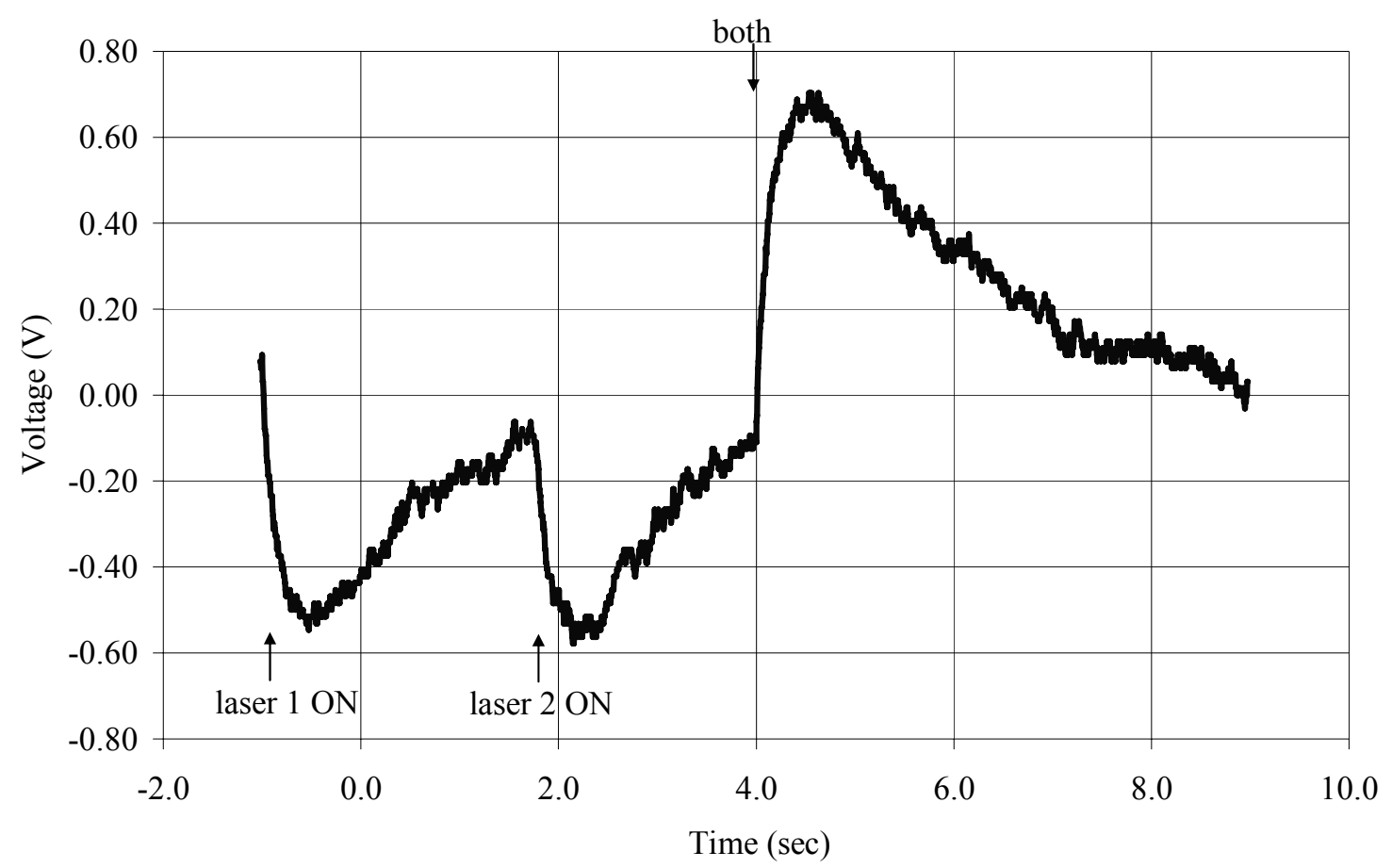

Figure 71. Unamplified $b R$ photoresponse with delayed activation of two lasers.

These results indicate that the photoresponse from the interaction between bR photocells in series and parallel was analogous to capacitors in series and parallel. A single bR photocell flashed with one laser or two lasers behaves similarly to bR photocells in parallel.

\subsection{SPA 4200 Measurements}

The electronic characteristics of bR were now measured with the SPA 4200, to understand if biasing the EPD bR with current or voltage, would affect the electrical functionality. Figure 72 shows the current response versus time with 2 offset bias voltage 0 and $2 \mathrm{~V}$. This figure shows that biasing the bR with a voltage does not effect the photocurrent. Next, the photovoltage was measured at 5 different bias currents of $0,1,2$, 3 , and $4 \mathrm{pA}$. As shown in Figure 73, biasing the bR with a current causes a gradual increase in the baseline but it does not appear to effect the photovoltage. The gradual increase in the baseline resembles a capacitor charging and as expected, the larger the bias current the greater the slope because the larger bias current causes the capacitor to charge faster. 


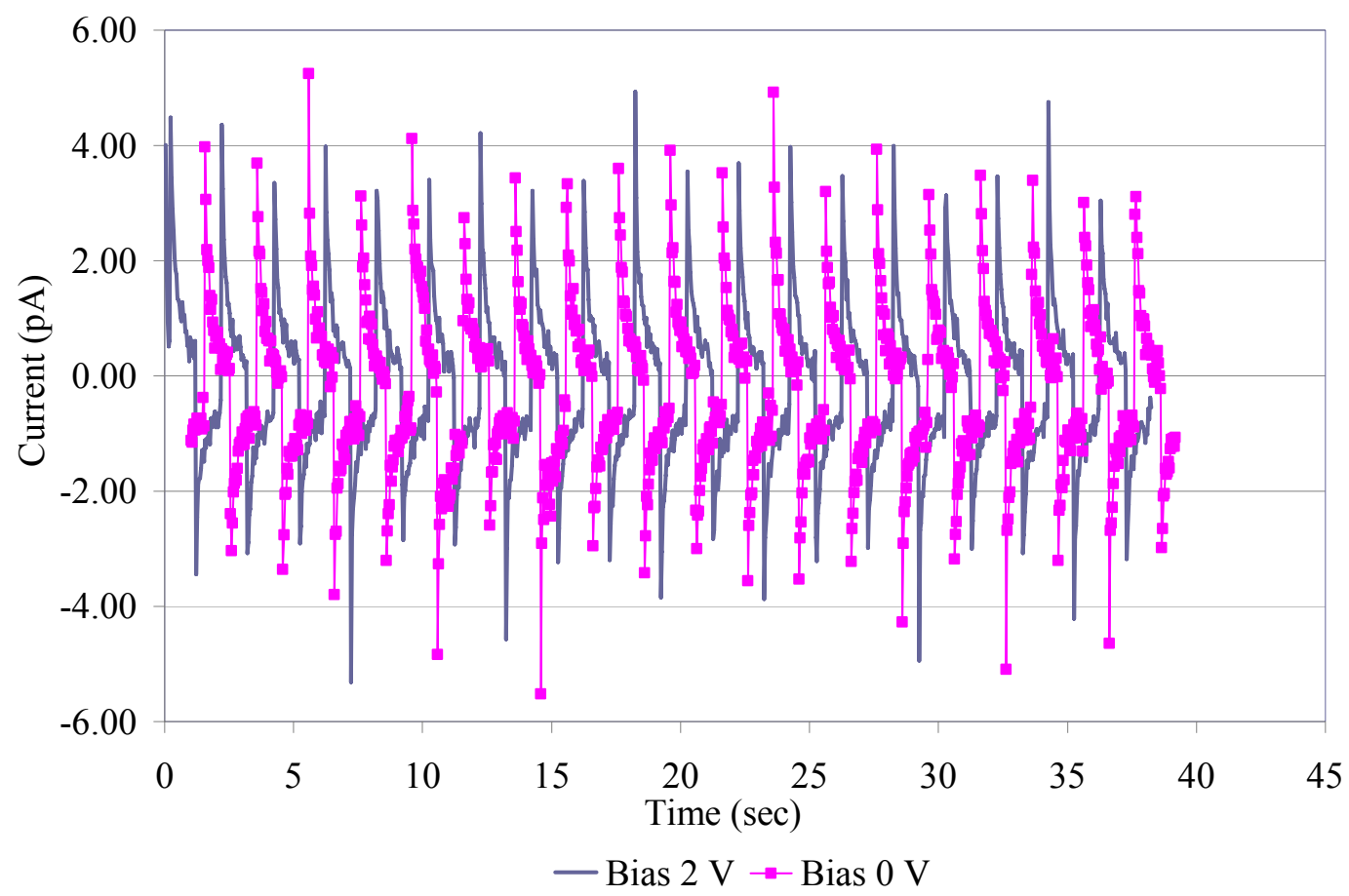

Figure 72. Current versus time at flash rate of $0.5 \mathrm{~Hz}$ with and without a voltage bias applied across the bR photocell during the flashing.

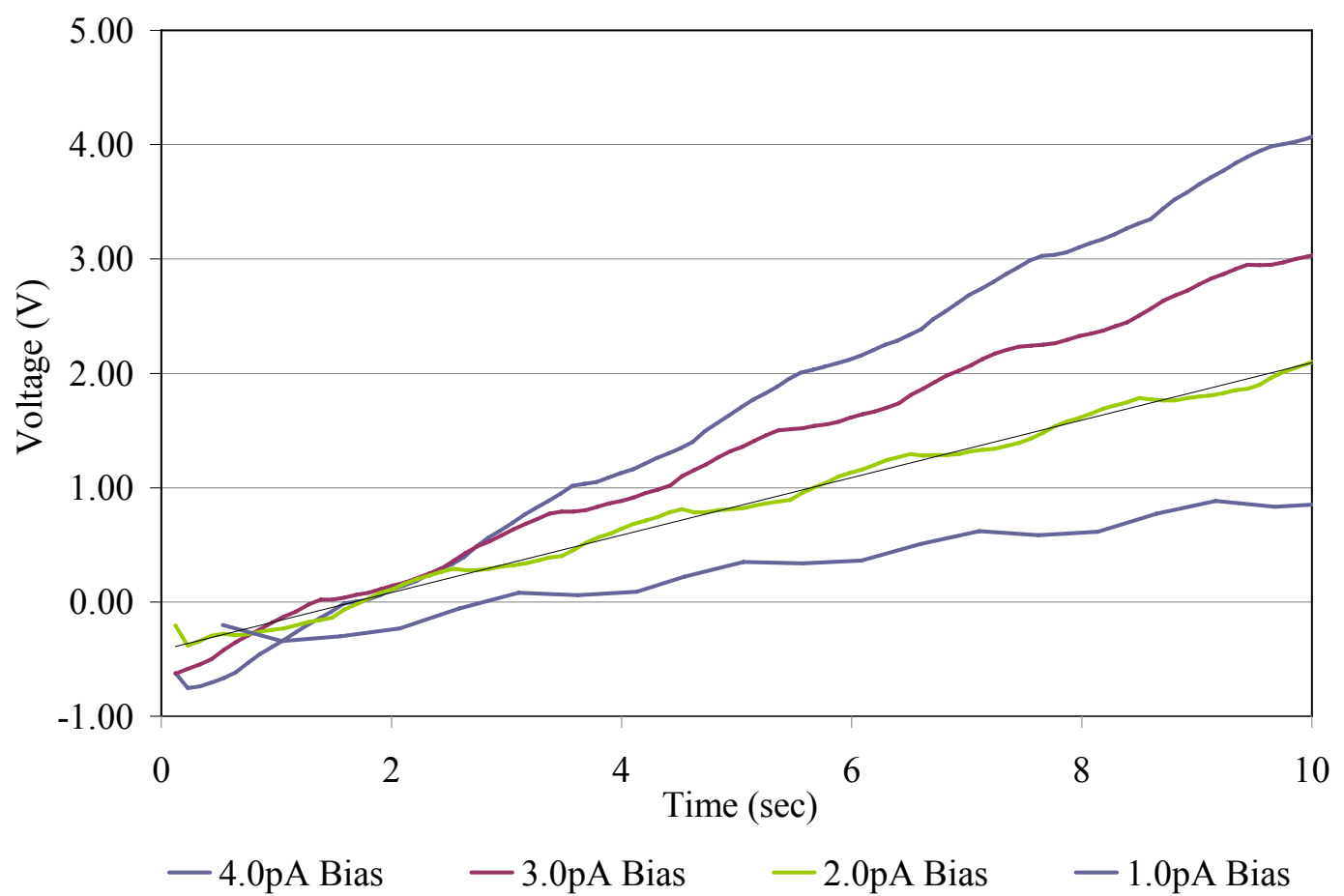

Figure 73. Voltage versus time at a flash rate of $0.5 \mathrm{~Hz}$ with varying current biases. 


\subsubsection{I-V Characteristic of a Multi-Monolayer bR Structure}

In this study, the I-V characteristics of $\sim 20$ LB monolayers of air-dried bR, were investigated. A top ITO electrode was placed on the multi-monolayer structure creating the bR photocell. Using the Keithley SPA 4200, I-V curves of the bR photocell were measured with the light-on and off. As shown in Figure 74, initial results reveal that there was a dramatic difference between the light activated and the non-light activated $b R$ photocell.

Assuming a linear relationship between the current and voltage, the resistance of the light-off bR photocell was estimated. For the non-light activated measurement the resistance per layer was $\sim 700 \mathrm{ohms}$ at room temperature and $\sim 50 \% \mathrm{RH}$. This measurement was consistent with Hwang et al. [17] for a dried monolayer structure. With light activation, the I-V curve resembles a diode characteristic curve as shown in Figure 75.

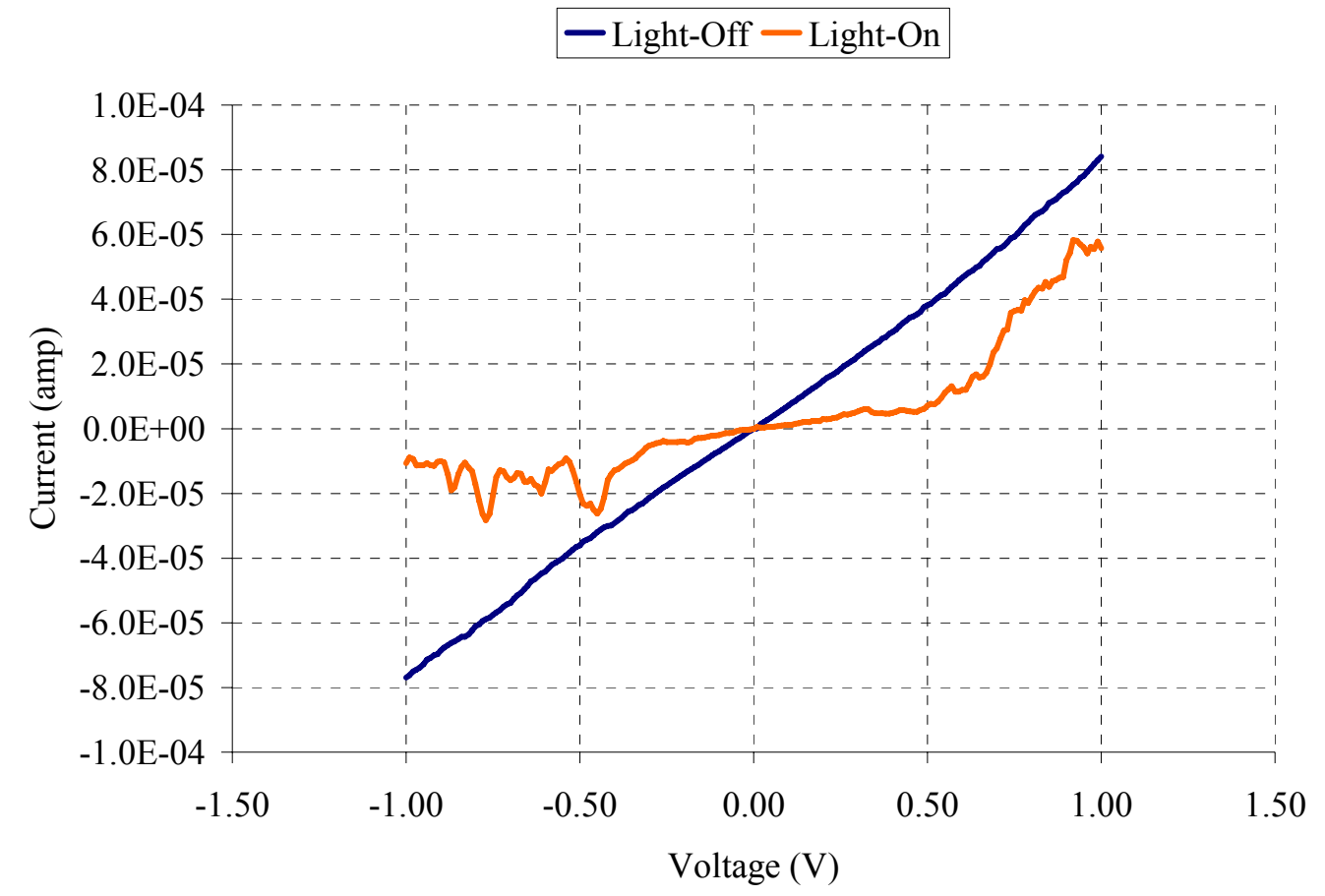

Figure 74. I-V characteristics of multi-monolayer bR structure with light activation and without. 


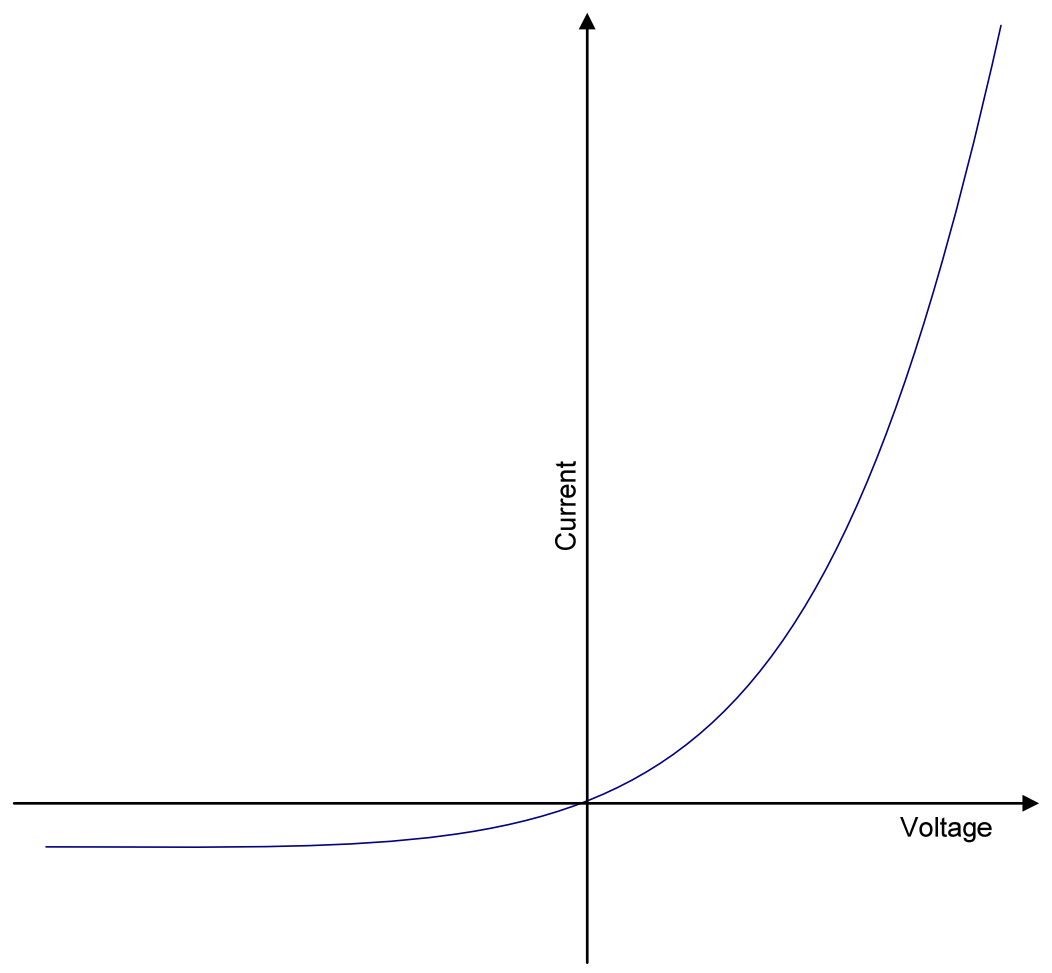

Figure 75. Idealized I-V curve for a p-n diode. 


\section{Chapter 7 Fabrication of Single Electron Transistors}

The combination of sputtering, photolithography, and focused ion beam (FIB) processing were used to fabricate single electron transistors with a bR interface pad. In this work the FIB was used to create the nano features while sputtering and photolithography were used for mircostructure fabrication. The FIB fabrication of the SETs was based on the work of Karre [86] and Acharya [87]. Due to wearing of the apertures of the FIB, the method [86] developed had to be re-created to deposit tungsten quantum islands $<10 \mathrm{~nm}$ in diameter. This chapter presents an overview of the FIB and the methods used to fabricate SETs with the bR interface pad.

\subsection{Focused Ion Beam Technology}

The FIB is an etching, deposition, and imaging system capable of fabricating micro/nanometer sized features. The FIB consists of seven main components: vacuum system; liquid metal ion source (LMIS); ion column; stage; detectors; gas inlet; and computer as shown in Figure 76. Just as the name implies the FIB focuses ions to etch, deposit, and image materials. The source of the ions is the LMIS. Generally the LMIS is gallium $\left(\mathrm{Ga}^{+}\right)$because of its low melting temperature. After heating the gallium a large electric field $\left(10^{8} \mathrm{~V} / \mathrm{cm}^{2}\right)$ is applied from the extractor electrode to the molten gallium forming a Taylor cone with a tip diameter of several nanometers. The large electric field also ionizes the $\mathrm{Ga}^{+}$from the tip of the Taylor cone creating the stream of $\mathrm{Ga}^{+}$ions. Next, stray ions are filtered as they pass through an aperture. Apertures vary in diameter as shown in Table 2. Larger apertures allow more ions to pass creating a larger beam current and the converse is true for a small aperture. Then the stream of ions are accelerated, aligned, and focused through a series of condenser lenses. The rate of fabrication of structures is based on the interaction of $\mathrm{Ga}^{+}$ions with the surface of the sample for etching, and the gas precursors for deposition.

The FIB is a proof of concept tool before mass production. With this tool it is possible for integrated circuit developers to modify circuits quickly instead of having to redesign photolithography masks which may take weeks [88]. The main problems with FIB-based fabrication are: etch and deposition rates are slow compared to other methods of etching and deposition; no real time imaging of etching and deposition with single beam systems; cost of operation; and the system requires frequent maintenance to maintain consistent operating conditions. 


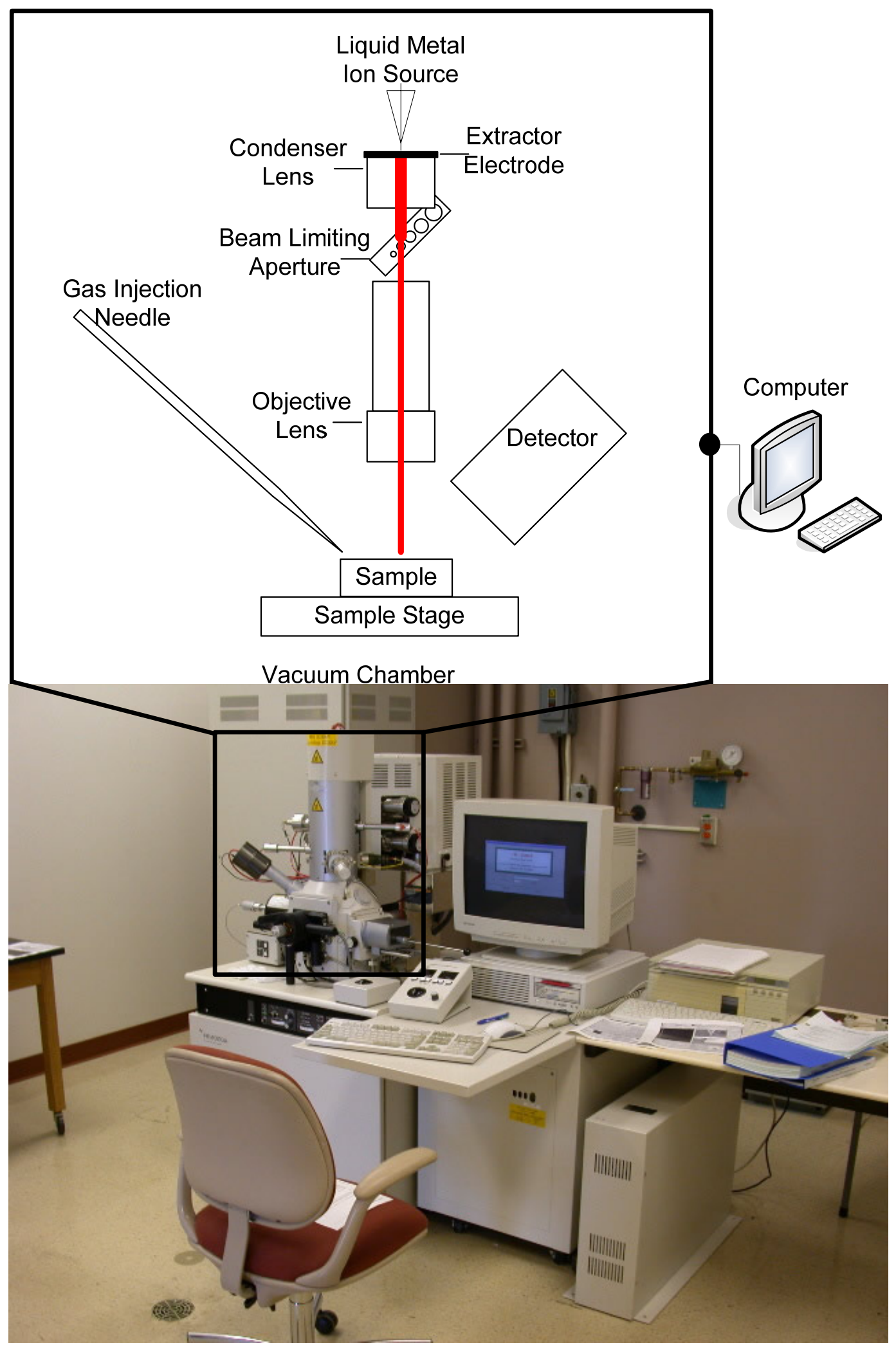

Figure 76. Hitachi FB-2000A focused ion beam system at Michigan Tech and schematic of general features of the FIB. 


\subsubsection{Etching Using the FIB}

The FIB is capable of etching features in the micro and nano regimens. The minimum feature size for each beam is determined by the beam current (i.e. smaller beam current, smaller feature size). For example beam M0-20, which has a beam current $\sim 0.004 \mathrm{nA}$, is capable of etching features as small as $11 \mathrm{~nm}$. Beam M1-500 has a beam current $29 \mathrm{nA}$, and can etch of material away quickly when compared to beam M0-20, but is limited to etching features in the micro-regimen.

The fabrication of nanostructure features without a dual beam system (containing both a FIB and SEM) is difficult since etched structures can not be easily imaged with the FIB without destroying the structure. FIB-based imaging etches more than SEM-based imaging, so, the nanostructure is more likely to be destroyed when imaging with the FIB than a SEM.

For etching to occur, the kinetic energy of the $\mathrm{Ga}^{+}$ions must be greater then the surface binding energy of the sample [88]. As shown in Table 2, there were seven different apertures ranging in diameter from $200 \mu \mathrm{m}-6 \mu \mathrm{m}$ and two different modes of operation ( 1 and 0 ). Mode 1 is primarily used for etching micro-sized features but can be used for deposition and imaging. Mode 0 is primarily used for imaging and deposition but can be used for etching nano-sized features.

Table 2. Beam specifics for the FB-2000A FIB at Michigan Tech.

\begin{tabular}{|c|c|c|c|c|c|}
\hline $\begin{array}{c}\text { Beam } \\
\text { Name }\end{array}$ & $\begin{array}{c}\text { Lens } \\
\text { Mode }\end{array}$ & $\begin{array}{c}\text { Aperture } \\
\text { Diameter } \\
(\mu \mathrm{m})\end{array}$ & $\begin{array}{c}\text { Specified Beam } \\
\text { Current (nA) }\end{array}$ & $\begin{array}{c}\text { Actual Beam } \\
\text { Current (nA) }\end{array}$ & Main Usage \\
\hline L. Scan & & & & & Low-mag observation \\
\hline M1-500 & Mode 1 & 500 & $11.000-15.000$ & $\sim 30$ & Etching and Imaging \\
\hline M1-300 & Mode 1 & 300 & $4.000-8.000$ & $\sim 9.7$ & Etching and Imaging \\
\hline M1-200 & Mode 1 & 200 & $2.000-3.500$ & $\sim 4$ & Etching and Imaging \\
\hline M1-100 & Mode 1 & 100 & $0.400-0.800$ & $\sim 0.8$ & Etching and Imaging \\
\hline M1-50 & Mode 1 & 50 & $0.100-0.300$ & $\sim 0.2$ & Etching and Imaging \\
\hline M1-20 & Mode 1 & 20 & $0.015-0.040$ & ${ }^{*}$ DU & Etching and Imaging \\
\hline M1-6 & Mode 1 & 6 & $0.001-0.005$ & ${ }^{*}$ DU & Etching and Imaging \\
\hline M0-200 & Mode 0 & 200 & $0.400-0.800$ & $\sim 0.8$ & Depositing and Imaging \\
\hline M0-100 & Mode 0 & 100 & $0.100-0.300$ & $\sim 0.2$ & Depositing and Imaging \\
\hline M0-50 & Mode 0 & 50 & $0.020-0.050$ & $\sim 0.03$ & Imaging, Etching, and Depositing \\
\hline M0-20 & Mode 0 & 20 & $0.004-0.010$ & $\sim 0.004$ & Imaging, Etching, and Depositing \\
\hline M0-6 & Mode 0 & 6 & $0.000-0.002$ & ${ }^{*}$ DU & Imaging, Etching, and Depositing \\
\hline
\end{tabular}

${ }^{*} \mathrm{DU}$ indicates beams that were not used is this research

\subsubsection{Deposition of Tungsten Quantum Islands Using FIB}

The FIB is not only capable of imaging and etching materials, but also able to deposit materials and form three dimensional nanostructures. [89, 90]. With FIB deposition it is possible to deposit both conductive materials as well as insulators $[88,91]$. The deposition is based on the ion beam interaction with the gas precursor, this type of 
deposition is called chemical vapor deposition. The rate of deposition is dependent on many factors. The main factors that effect deposition rate were gas precursor density at the surface of the sample and the ion beam interaction with the gas precursor. Sub factors that effect the rate of deposition include dwell time (ion beam interaction duration at a point), interlace (the amount of ion beam overlap for each point), deposition gun height above the sample, deposition gun diameter, precursor heating temperature, and beam current. These factors were all important when characterizing a deposition process.

At Michigan Tech the FIB is capable of depositing tungsten. Tungsten hexacarbonyl $\mathrm{W}(\mathrm{CO})_{6}$ is heated above its vaporization temperature forming $\mathrm{W}(\mathrm{CO})_{6}$ gas precursor and it is ready for deposition. The valve is then opened and the $\mathrm{W}(\mathrm{CO})_{6}$ gas begins to flow out of the needle onto the surface of the substrate and the deposition begins as the beam interacts with the gas precursor. The computer controlled ion beam allows for complex geometry fabrication.

\subsection{SET Fabrication with Interface Pad for Bacteriorhodopsin}

The SETs with the bR interfacing pads were fabricated by combination of sputtering, photolithography, and FIB-based etching and deposition. SETs were composed of a gate, drain, source, gap, quantum islands, and tunneling junction. The electrodes and pads for the gate, drain, and source can easily be fabricated by photolithographic processing, reducing FIB time and the time it takes to fabricate these features with the FIB. The gap and quantum islands were fabricated with the FIB.

A baseline procedure was established but as the process evolved or problems were encountered the process had to be modified. As shown in Figure 77, the fabrication of the bR-SET began with a bare silicon wafer. The wafer was chemically cleaned in Piranha solution of sulfuric acid and hydrogen peroxide (3:1). After cleaning the wafer, a substrate isolation layer of $\mathrm{Al}_{2} \mathrm{O}_{3}$ was deposited using a Perkin-Elmer 2400-8J parallel plate RF sputter system with the parameters shown in Table 3. A conductive layer of chromium was deposited using the Perkin-Elmer 2400-6J parallel plate RF sputter system with the parameters shown in Table 4.

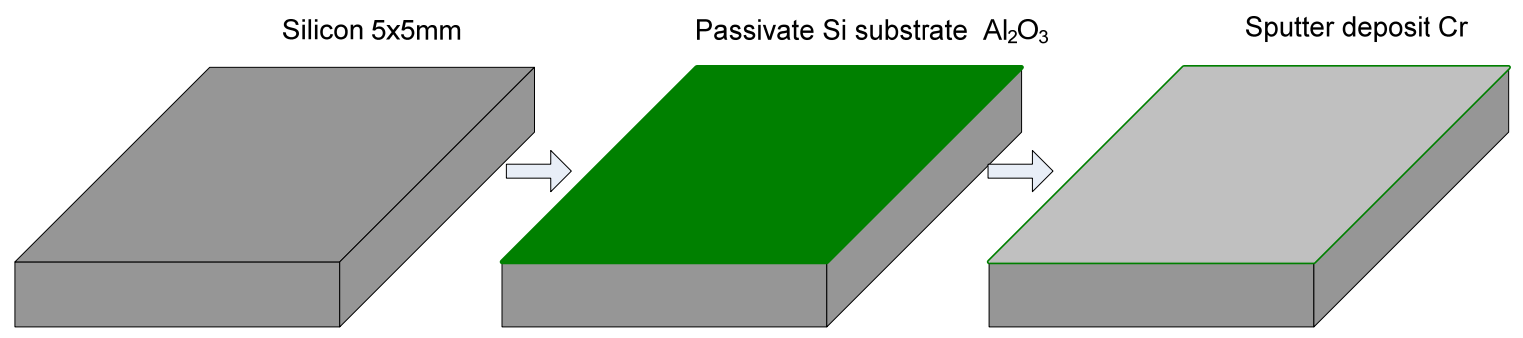

Figure 77. bR-SET fabrication procedure. 
Table 3. RF sputter deposition parameters for $\mathrm{Al}_{2} \mathrm{O}_{3}$.

\begin{tabular}{|l|l|}
\hline Process Parameters & \multicolumn{1}{|c|}{ Material } \\
\hline Tool & Perkin-Elmer 2400-8J \\
\hline Deposition material & $\mathrm{Al}_{2} \mathrm{O}_{3}$ \\
\hline RF power & $1000 \mathrm{~W}$ \\
\hline DC bais & $0-100 \mathrm{~V}$ \\
\hline Substrate bais & $0 \mathrm{~V}$ \\
\hline Process pressure & $8-9$ mTorr \\
\hline $\begin{array}{l}\text { Gas flowed during the } \\
\text { deposition }\end{array}$ & $\begin{array}{l}\mathrm{Ar}: 20.0 \mathrm{SCCM} \quad \text { Oxygen: } \\
4.0 \text { SCCM }\end{array}$ \\
\hline Depositon duration & 30 minutes \\
\hline
\end{tabular}

Table 4. RF sputter deposition parameters for C.

\begin{tabular}{|l|l|}
\hline Process Parameters & \multicolumn{1}{|c|}{ Material } \\
\hline Tool & Perkin-Elmer 2400-6J \\
\hline Deposition material & $\mathrm{Cr}$ \\
\hline RF power & $100 \mathrm{~W}$ \\
\hline DC bais & $0-10 \mathrm{~V}$ \\
\hline Substrate bais & $0 \mathrm{~V}$ \\
\hline Process pressure & $8-9$ mTorr \\
\hline $\begin{array}{l}\text { Gas flowed during the } \\
\text { deposition }\end{array}$ & Ar: 10.0 SCCM \\
\hline Depositon duration & 2.5 minutes \\
\hline
\end{tabular}

As shown in Figure 78 photoresist was spun, exposed, and developed on the $\mathrm{Cr} / \mathrm{Au}$ coated substrate as described in sequential order in Table 5. The Cr layer with the patterned PR was placed in a wet etchant, Cr-7S10, for 30 seconds and then rinsed in DDI water. To ensure that the Cr layer was removed a digital multimeter was used to check the resistance of the etched area. Once the resistance was significantly large (50 Mohms limits of the multimeter) the photoresist was stripped. The PR was stripped by soaking the substrate in acetone and then isopropanol to remove PR residue.

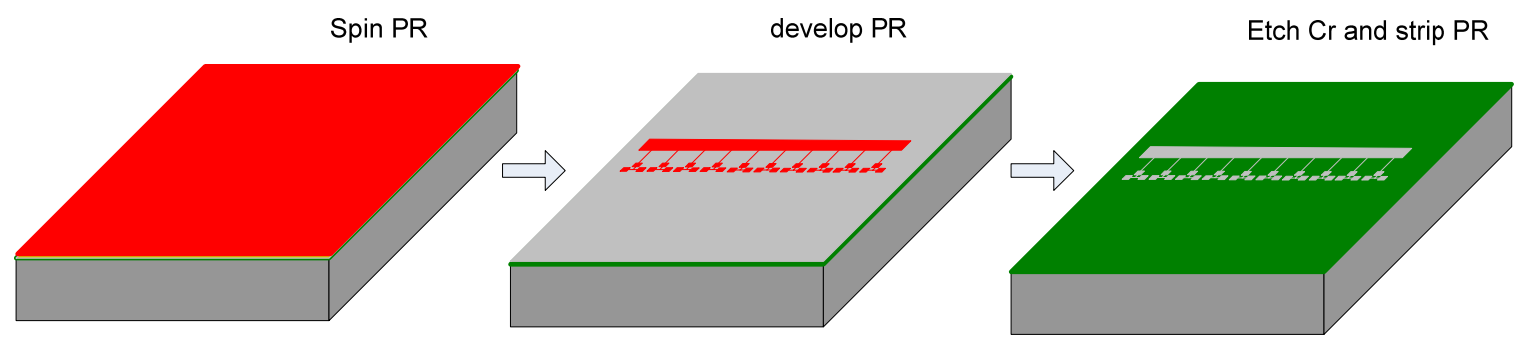

Figure 78. bR-SET fabrication procedure. 
Table 5. Postive Futurrex photoresist processing.

\begin{tabular}{|l|l|}
\hline Process Parameters & \multicolumn{1}{|c|}{ Material } \\
\hline Photoresist & Positive Futurrex \\
\hline Spin rate & 6000 RPM \\
\hline Spin duration & 15 seconds \\
\hline Soft bake 1 minute & $120^{\circ} \mathrm{C}$ \\
\hline EV aligner UV power & $\sim 9 \mathrm{~mW}$ \\
\hline PR exposure & 15 seconds \\
\hline Post bake 1 minute & $120^{\circ} \mathrm{C}$ \\
\hline RD6 developer & 15 seconds \\
\hline Hard bake 5 minutes & $100^{\circ} \mathrm{C}$ \\
\hline
\end{tabular}

As shown in Figure 79 the next steps were to spin-on, and develop the PR, FIB etch the gap, and deposit the tungsten dots. A sacrificial layer of high temperature negative photoresist, NR1-3000PY, was patterned on the Cr pads except at the junction where the source/drain/gate merge. A negative high temperature resist was chosen because it is designed for lift-off and capable of withstanding process temperatures up to $180^{\circ} \mathrm{C}$.

After patterning the sacrificial layer of PR the FIB was used to trim the electrodes to a width of $\sim 250 \mathrm{~nm}$ and the "T" shaped gap where the source/drain/gate merge as shown in Figure 80. To cut the gap beam M0-20 with a beam current of $0.04 \mathrm{nA}$, area of 4 , zoom of 2 , line width of 1 , dwell time of $30 \mu \mathrm{s}$, frame number of 70, was repeated 2-3 times to cut the gap. The FIB was then used to deposit tungsten QDs as described in Table 7.

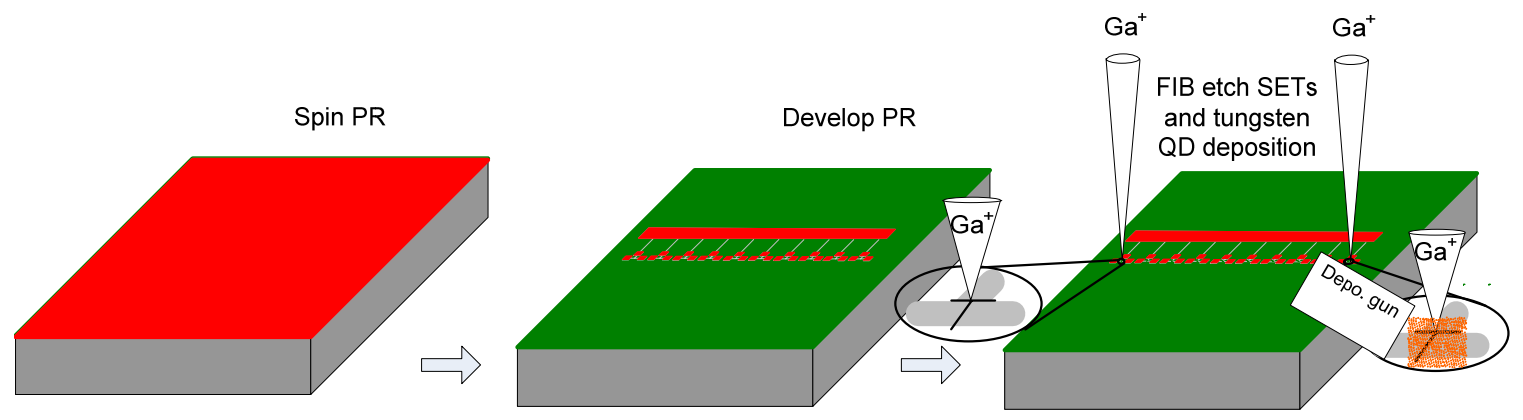

Figure 79. bR-SET fabrication procedure. 
Table 6. Negative Futurrex photoresist processing.

\begin{tabular}{|l|l|}
\hline Process Parameters & \multicolumn{1}{|c|}{ Material } \\
\hline Photoresist & Negative Futurrex NR1-3000PY \\
\hline Spin rate & 3000 RPM \\
\hline Spin duration & 40 seconds \\
\hline Soft bake 1 minute & $150^{\circ} \mathrm{C}$ \\
\hline EV aligner UV power & $\sim 9 \mathrm{~mW}$ \\
\hline PR exposure & 120 seconds \\
\hline Post bake 1 minute & $100^{\circ} \mathrm{C}$ \\
\hline RD6 developer & 25 seconds \\
\hline Hard bake 5 minutes & $100^{\circ} \mathrm{C}$ \\
\hline
\end{tabular}

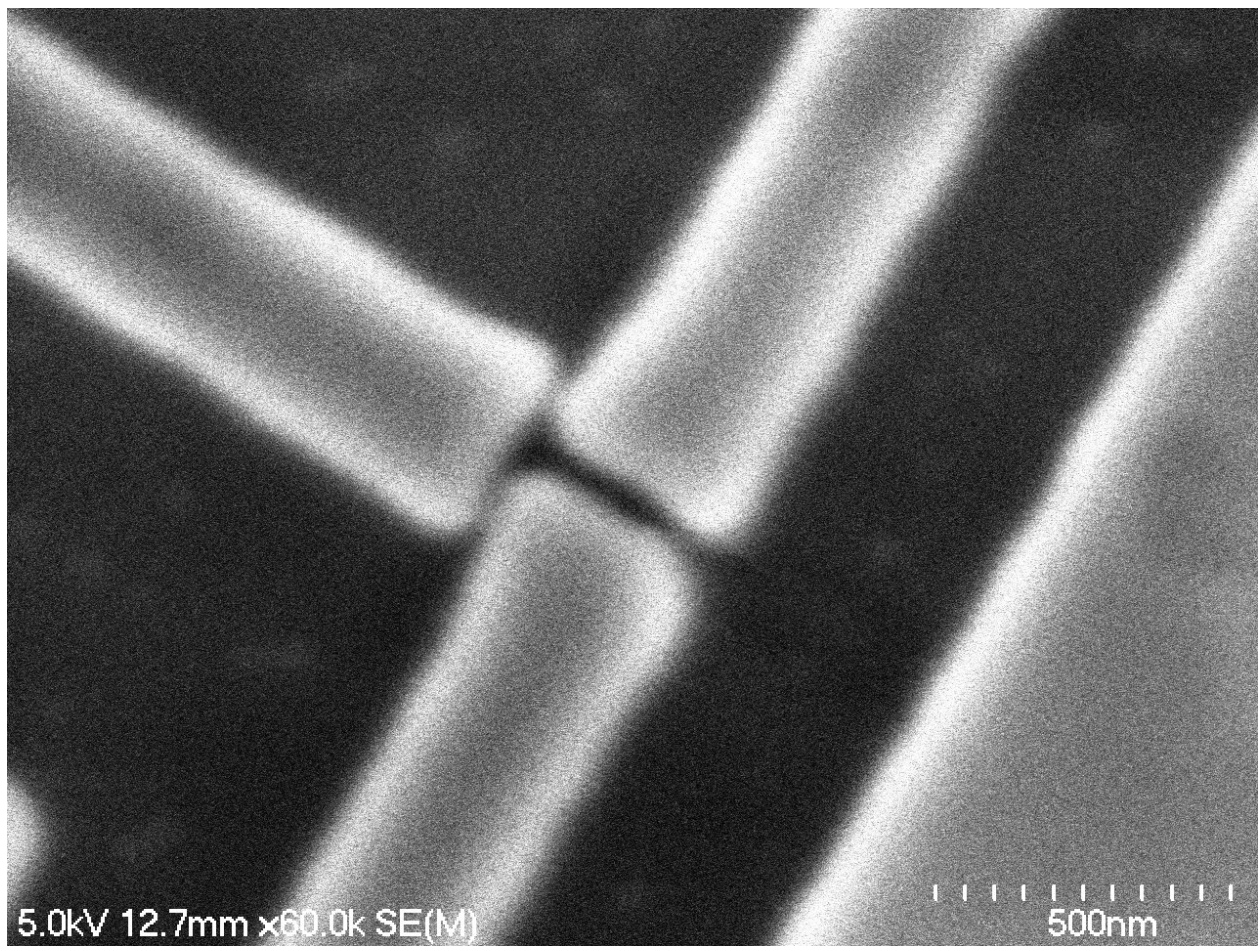

Figure 80. FIB etched gap.

Table 7. Initial tungsten QD deposition parameters.

\begin{tabular}{|l|l|}
\hline Process Parameters & \multicolumn{1}{|c|}{ Fabrication Mode } \\
\hline Beam & M1-200 \\
\hline Beam Current & $4.0 \mathrm{nA}$ \\
\hline Area of deposition & $16.062 \times 16.062 \mu \mathrm{m}$ \\
\hline Dwell time & $9 \mu \mathrm{S}$ \\
\hline Interlace & 4 \\
\hline Deposition Gun Parameters \\
\hline Heater Setting & 60 \\
\hline & \\
Location above sample & $3-4$ clicks \\
\hline
\end{tabular}


Following FIB processing, the tungsten QDs were wet chemically oxidized in peracetic acid for 2 minutes. Peracetic acid was created by combining equal parts of $30 \%$ hydrogen peroxide and $80 \%$ acetic acid. The samples were then rinsed in DDI water to remove any residual peracetic acid and dried with high purity nitrogen.

In order to protect and isolate the devices a passivation layer of $\mathrm{Al}_{2} \mathrm{O}_{3}$ was $\mathrm{RF}$ sputter deposited as described in Table 8 as shown in Figure 81. Lift-off was then performed with Futurrex stripper RR5, heated to $100{ }^{\circ} \mathrm{C}$ to remove the sputtered $\mathrm{Al}_{2} \mathrm{O}_{3}$ on the pads of the devices. The devices were then checked for functionality with the Keithley 4200 SPA. If there were functioning devices a second sacrificial layer of PR was spun and developed.

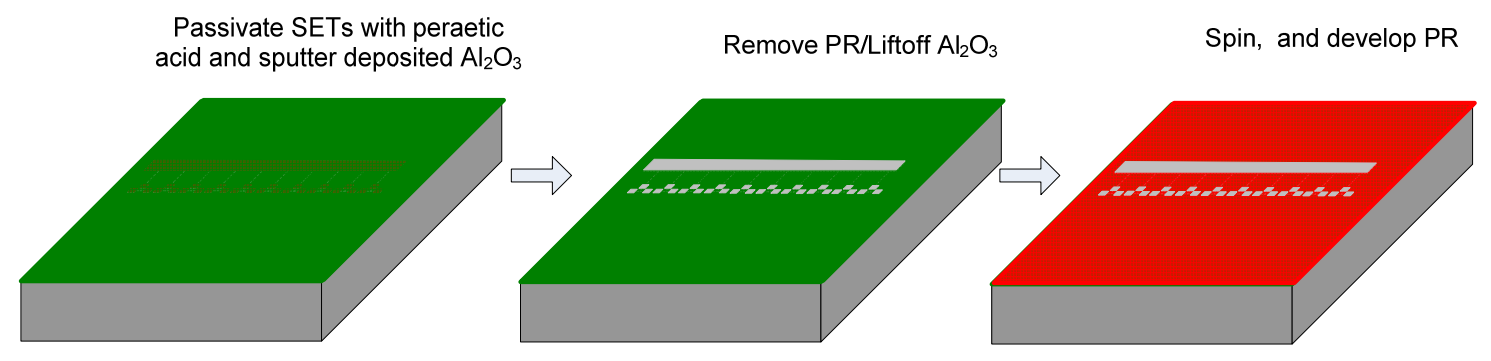

Figure 81. bR-SET fabrication procedure

Table 8. RF sputter deposition parameters for SET passivation layer.

\begin{tabular}{|l|l|}
\hline Process Parameters & \multicolumn{1}{|c|}{ Material } \\
\hline Tool & Perkin-Elmer 2400-8J \\
\hline Deposition material & $\mathrm{Al}_{2} \mathrm{O}_{3}$ \\
\hline RF power & $1000 \mathrm{~W}$ \\
\hline DC bais & $0-100 \mathrm{~V}$ \\
\hline Substrate bais & $0 \mathrm{~V}$ \\
\hline Process pressure & $8-9$ mTorr \\
\hline $\begin{array}{l}\text { Gas flowed during the } \\
\text { deposition }\end{array}$ & $\begin{array}{l}\text { Ar: } 20.0 \mathrm{SCCM} \\
\text { Oxygen: } 4.0 \mathrm{SCCM}\end{array}$ \\
\hline Depositon duration & 10 minutes \\
\hline
\end{tabular}

As shown in Figure $82, \mathrm{bR}$ at a concentration of $6.9 \mathrm{mg} \mathrm{mL}^{-1}$, was electrophoretically deposited on the gate by applying $4 \mathrm{~V}$ for 40 seconds across the $1.5 \mathrm{~mm}$ gap separating the gate electrode and the counter-electrode. A semitransparent film of gold was then RF sputter deposited on the bR as described in Table 9. The PR was then stripped away leaving the gold coated $b R$ on the gate pad. 


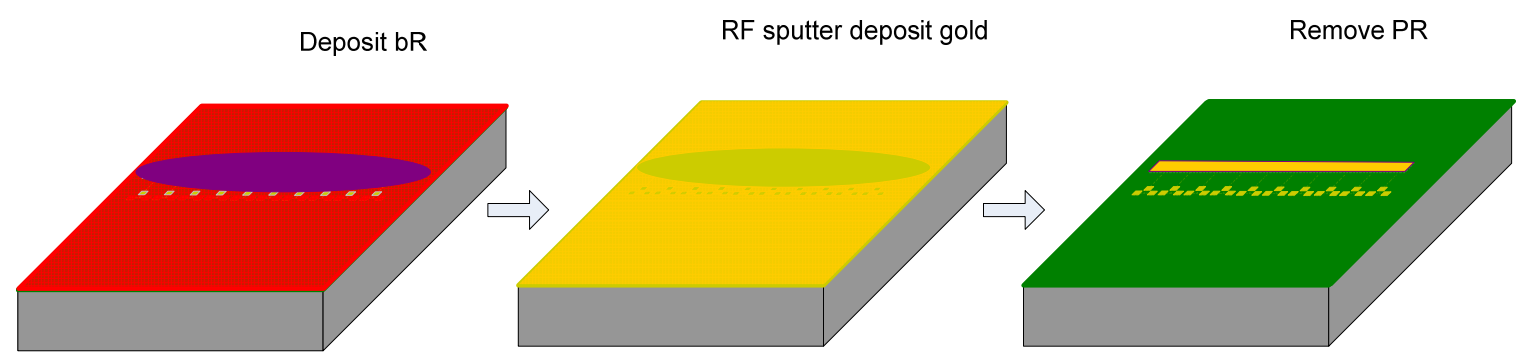

Figure 82. bR-SET fabrication procedure.

Table 9. Gold deposition parameters on bR.

\begin{tabular}{|l|l|}
\hline Process Parameters & \multicolumn{1}{|c|}{ Material } \\
\hline Tool & Perkin-Elmer 2400-6J \\
\hline Deposition material & $\mathrm{Au}$ \\
\hline RF power & $100 \mathrm{~W}$ \\
\hline DC bais & $0-100 \mathrm{~V}$ \\
\hline Substrate bais & $0 \mathrm{~V}$ \\
\hline Process pressure & $8-9$ mTorr \\
\hline $\begin{array}{l}\text { Gas flowed during the } \\
\text { deposition }\end{array}$ & Ar: $10.0 \mathrm{SCCM}$ \\
\hline Depositon duration & 30 seconds \\
\hline
\end{tabular}

\subsubsection{Changes of Initial Process}

\subsubsection{Burned Photoresist}

The first challenge encountered was that the sacrificial layer of high temperature photoresist was not lifting off the $\mathrm{Cr}$ pads after $\mathrm{Al}_{2} \mathrm{O}_{3}$ sputter deposition. With the use of in-situ temperature indictor strips, it was determined that the processing temperature was greater then $220{ }^{\circ} \mathrm{C}$, which exceeded the maximum temperature of the PR by $40{ }^{\circ} \mathrm{C}$. This was also confirmed by FESEM before the deposition as shown in Figure 83 and then after the deposition as shown in Figure 84. This problem was solved by decreasing the $\mathrm{RF}$ power from $1000 \mathrm{~W}$ to $750 \mathrm{~W}$ and increasing the sputtering duration to 15 minutes instead of 10 minutes to maintain the film thickness. After re-characterizing the $\mathrm{Al}_{2} \mathrm{O}_{3}$ sputtering system at 750 Watts it was determined that the deposition needed to be 15 minutes long to achieve approximately the same thickness of $\mathrm{Al}_{2} \mathrm{O}_{3}$ as sputtered at 1000 W. With the new deposition parameters the PR was now lifting-off after the $\mathrm{Al}_{2} \mathrm{O}_{3}$ deposition. In situ temperature indicator strips also revealed that the temperature was not exceeding $160{ }^{\circ} \mathrm{C}$ during the sputter deposition of $\mathrm{Al}_{2} \mathrm{O}_{3}$. This did not significantly alter the process flow for creating the bR-SETs. 


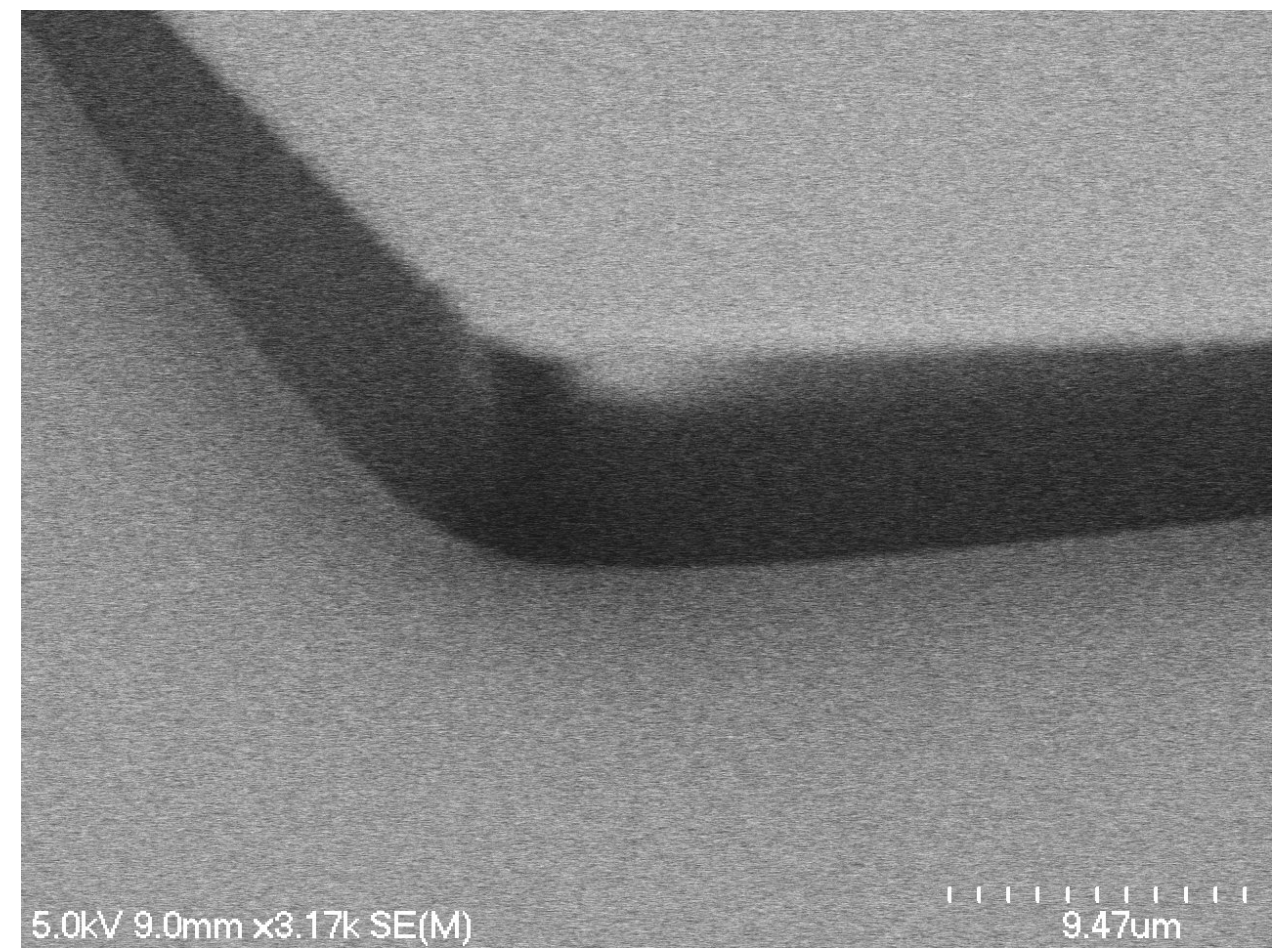

Figure 83. NR1-3000PY prior to $\mathrm{Al}_{2} \mathrm{O}_{3}$ sputter deposition.

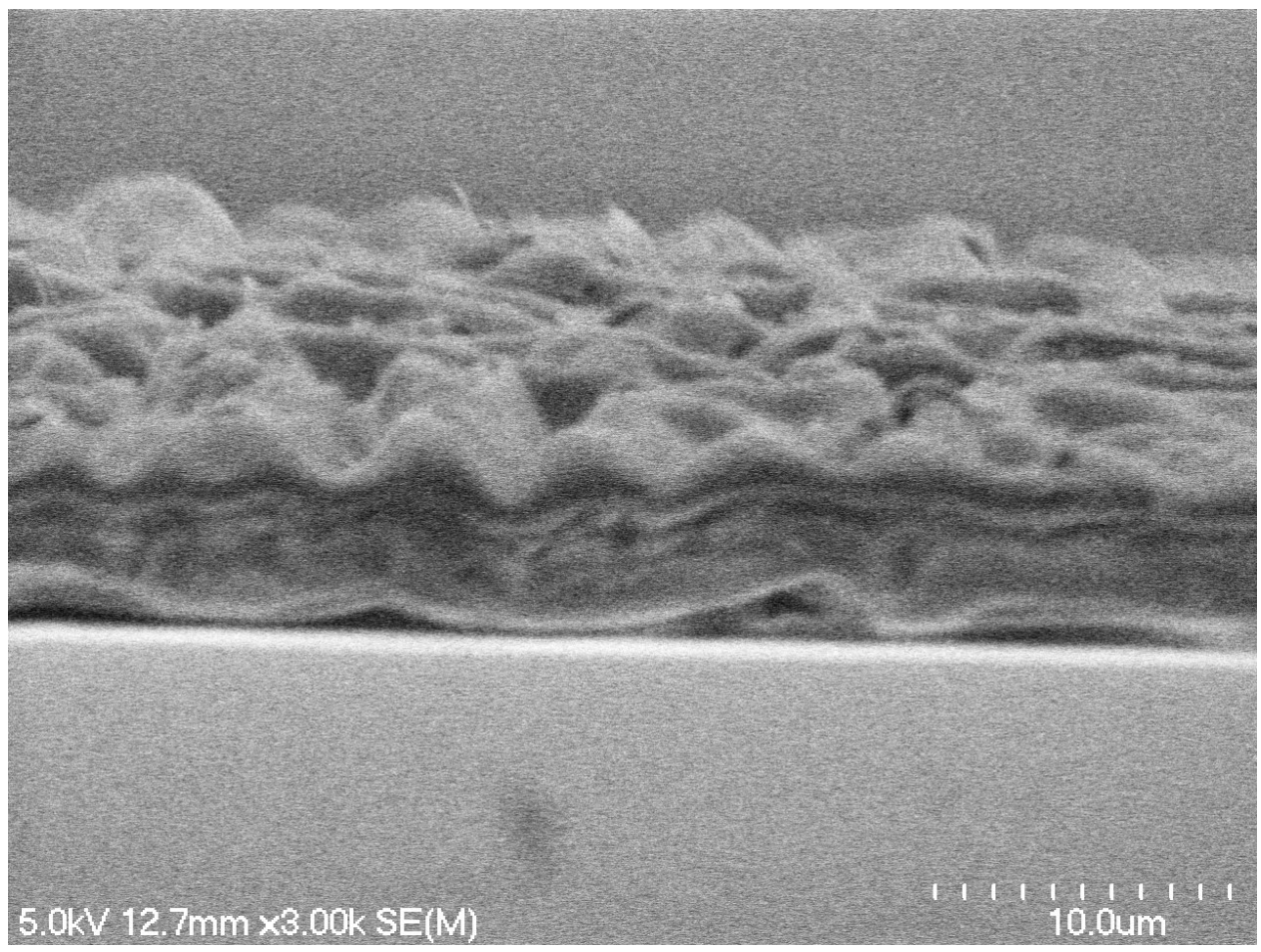

Figure 84. NR1-3000 after sputter deposition. 


\subsubsection{Residual Photoresist}

The next challenge was encountered while using an AFM to image the surface roughness of the $\mathrm{Cr}$. It was discovered that after stripping away the PR with acetone and isopropanol, a residue of PR was not being removed, as shown in Figure 85. This problem was solved by performing a base Piranha etch after stripping the PR. The recipe for the base Piranha etch was found at memsexhange.org. The base Piranha solution was a 3:1 ratio of ammonium hydroxide and hydrogen peroxide mixture. Samples were soaked in the solution for 5 minutes, rinsed in DDI water, and nitrogen dried. As shown in Figure 86 all/majority of the PR residue was removed following the base piranha etch. The additional cleaning step had no noticeable change on the functionality of the SETs.

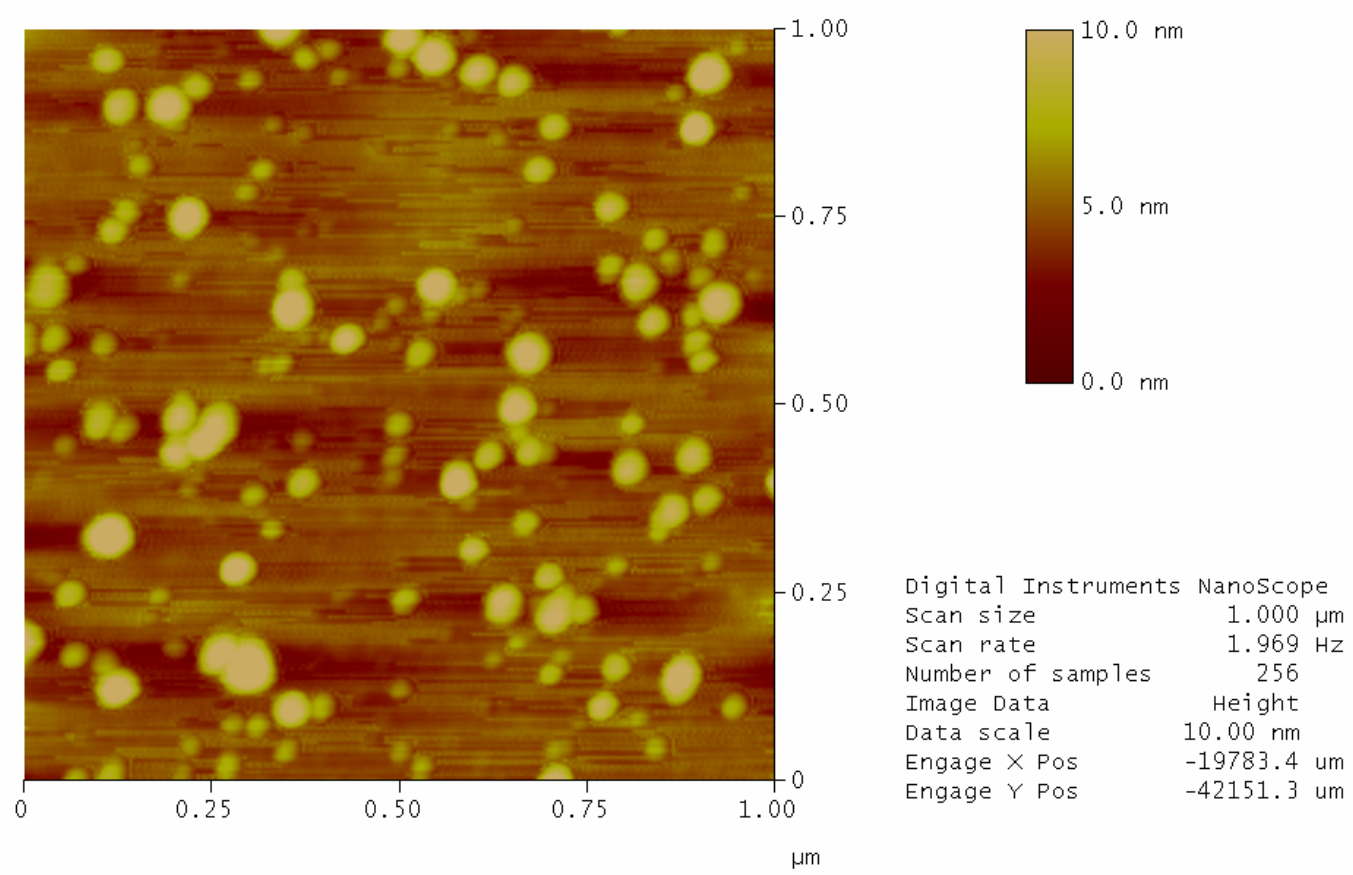

Figure 85. Substrate after cleaning with resist stripper. 

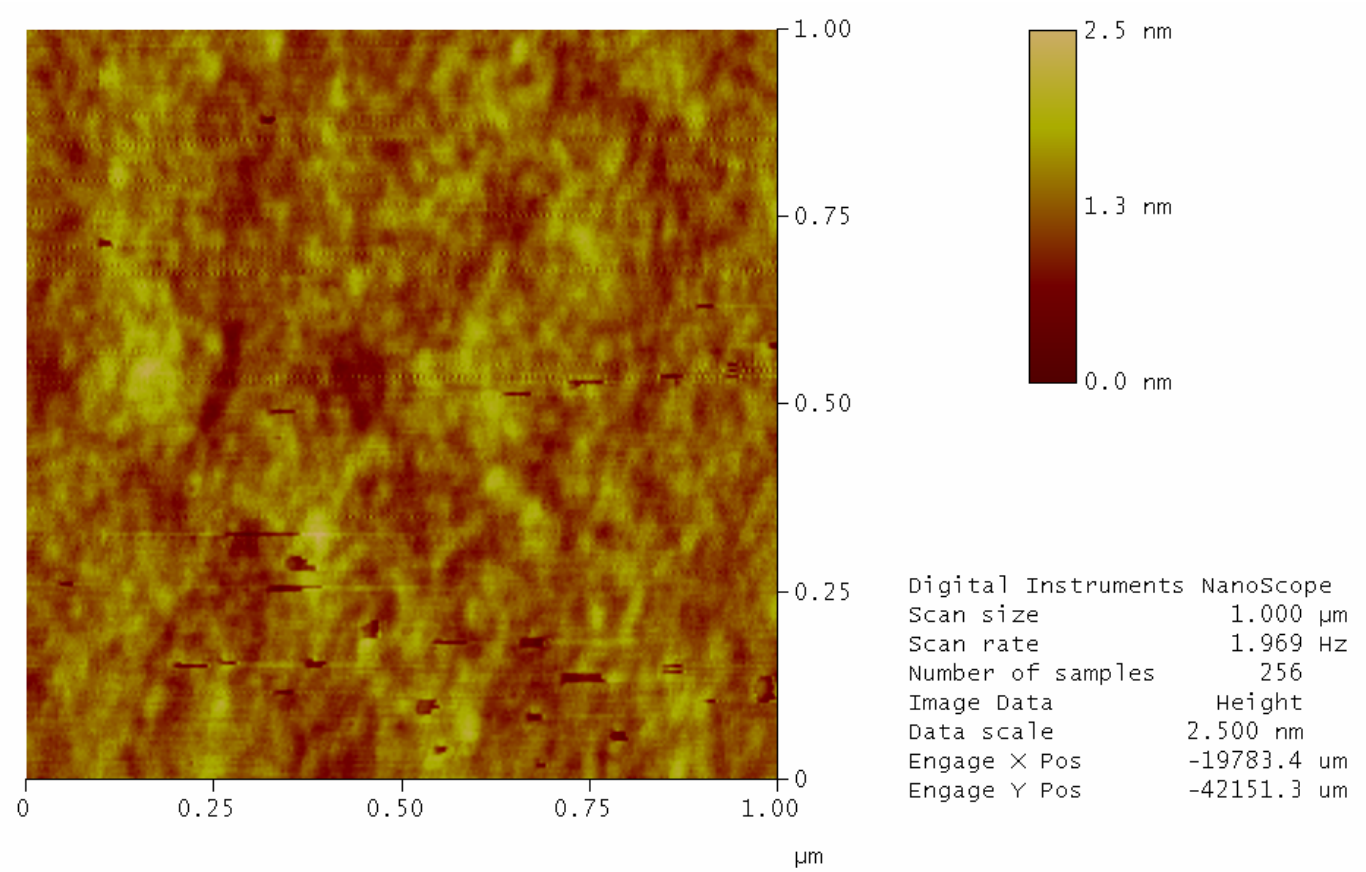

Figure 86 . Substrate after cleaning with Pirahana (three parts hydrogen peroxide $30 \%$ to one part ammonium hydroxide)

\subsubsection{Chromium Thin Film}

The next challenge encountered was the thin sputter deposited $\mathrm{Cr}$ film $(<25 \mathrm{~nm})$ as shown in Figure 77. As Kulkarni [92] determined, thin sputtered deposited films of $\mathrm{Cr}$ deposit with oxygen and carbon. In thin films $<25 \mathrm{~nm}$, the oxygen, carbon, and the structure of the film significantly affects the electrical properties. Figure 87 shows the I-V characteristic of $25 \mathrm{~nm}$ of sputter deposited $\mathrm{Cr}$, this curve indicates that there is not a good Ohmic contact between the probes, especially at low current levels (i.e. picoAmp range), where the SET characteristics would be measured. The curve also resembles the coulomb blockade region of an I-V of an SET but there was an obvious difference; the blockade region is not horizontal. For these reasons, the thin sputtered $\mathrm{Cr}$ was not an adequate electrode or pad for SETs.

These findings, resulted in a slight change in the process, from sputtered $\mathrm{Cr}$ as shown in Figure 77, to sputtered consecutive layers of $\mathrm{Cr}$ and then $\mathrm{Au}$, at a ratio of 1:5 ratio. The $\mathrm{Cr} / \mathrm{Au}$ layers were patterned through photolithography and a combination of wet chemical etchants. To etch the $\mathrm{Cr} / \mathrm{Au}$ layers, the sample was exposed to the gold etchant for 40 seconds, and then the Cr etchant, Cr-7S10, for 30 seconds as shown in Figure 88. The sputtered $\mathrm{Au} / \mathrm{Cr}$ has a much higher conductance as shown in Figure 89, a substantial improvement when compared to the Cr film shown in Figure 87.

With these changes implemented room temperature SET functionality was still not achieved repeatedly. The next variables checked were the tungsten dots and possible contamination caused by photolithographic processing of the SETs. 


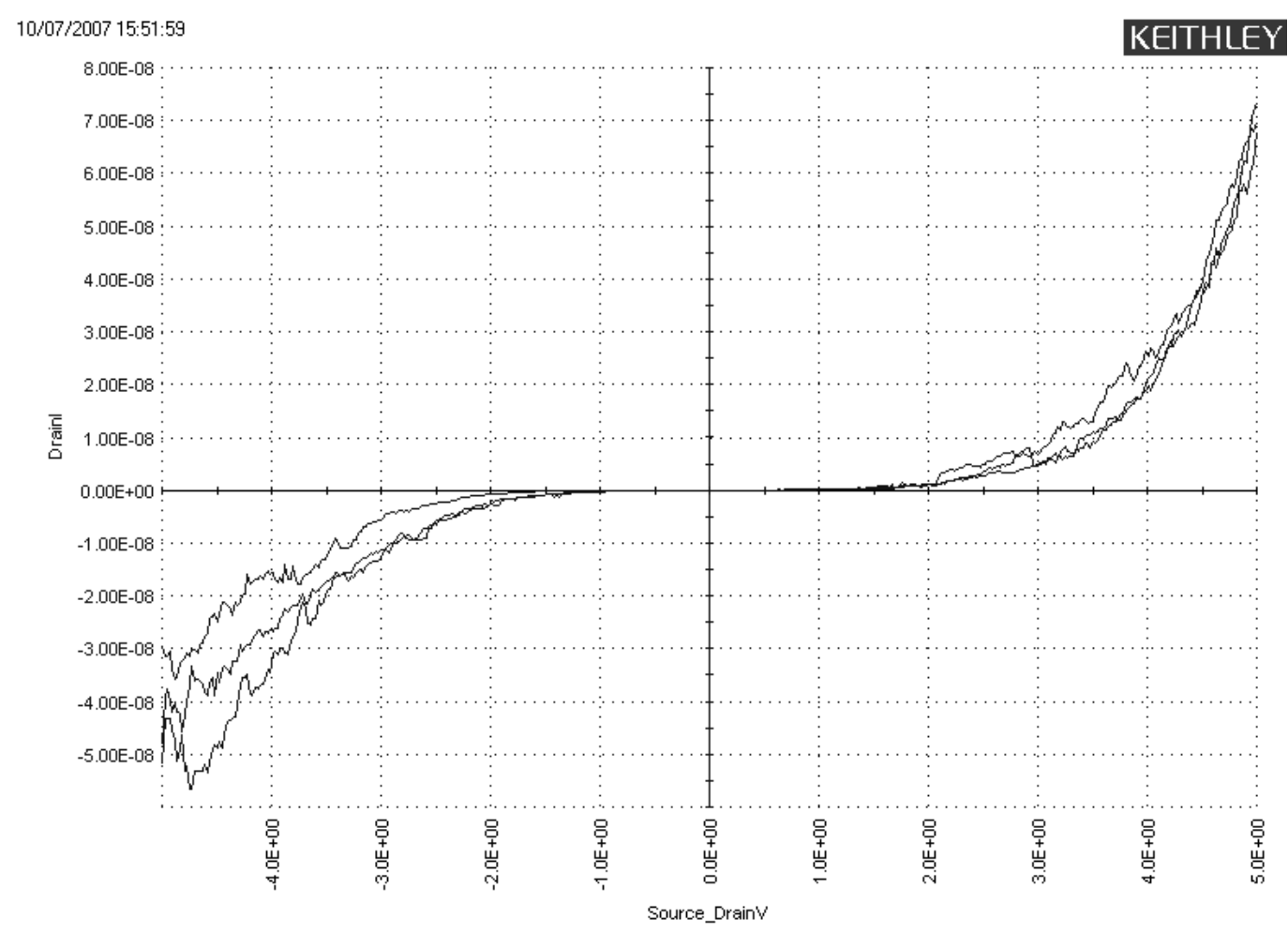

Figure 87. Measured I-V of $25 \mathrm{~nm}$ of sputter deposited Cr.

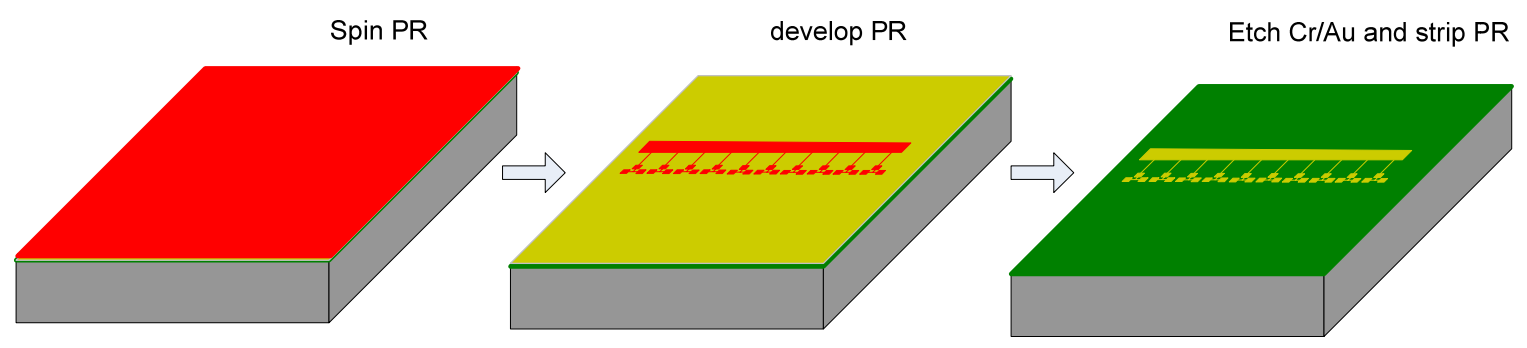

Figure 88. Modified process flow etching $\mathrm{Cr} / \mathrm{Au}$. 


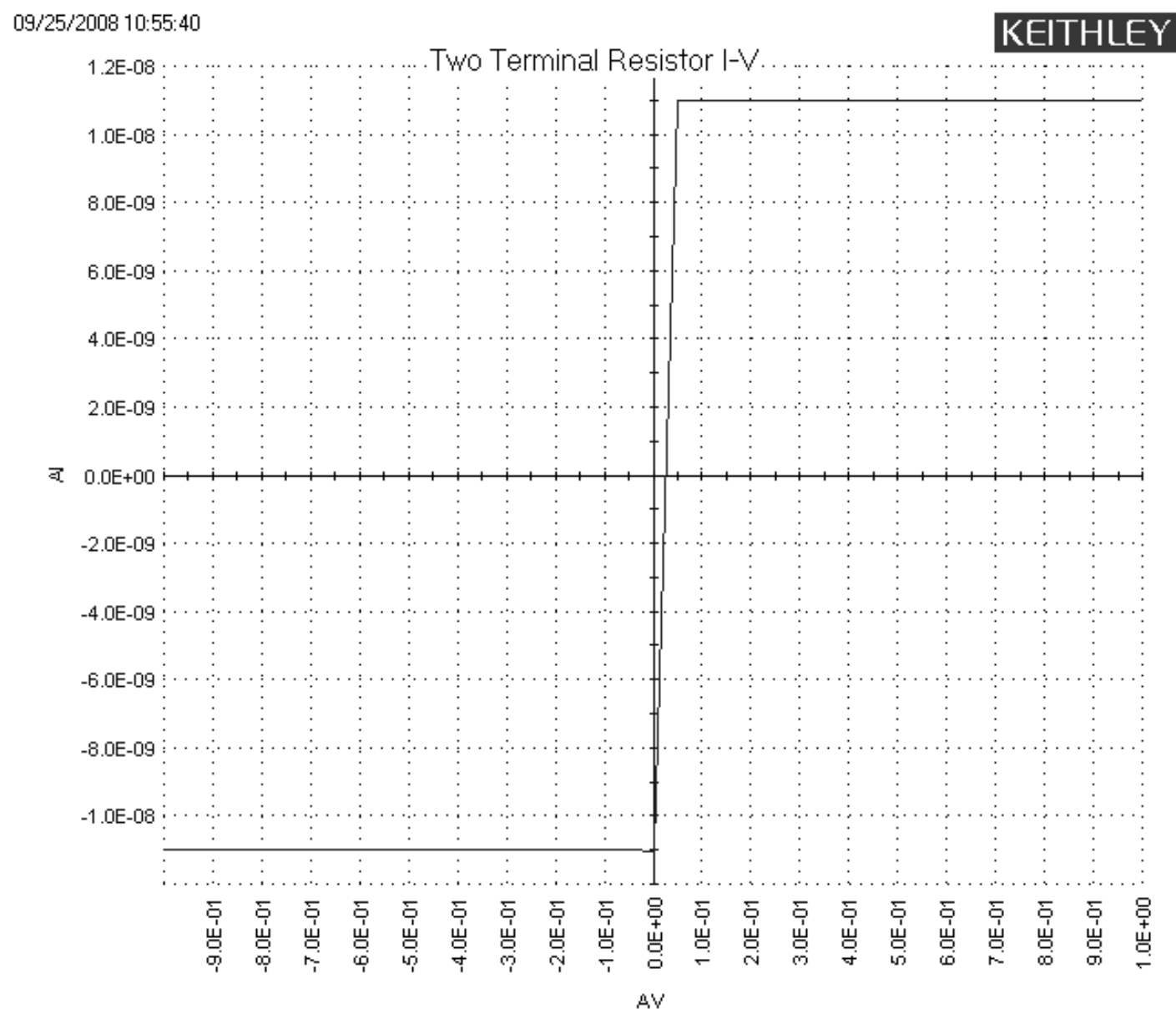

Figure 89 . Measured I-V of $\sim 5 \mathrm{~nm}$ of $\mathrm{Cr}$ with $\sim 20 \mathrm{~nm}$ gold.

\subsubsection{Analyses of SET Components}

Since the solutions to the previously described problems did not produce working SETs, the main focus shifted to the fabrication of the SETs. Essentially to achieve room temperature SETs, the charging energy $\left(E_{c}\right)$ of the device must be greater than the thermal energy $\left(E_{k}\right)$. To achieve the device capacitance must be in the attofarad regimen. The overall device capacitance can be reduced; by decreasing the QD to $<10 \mathrm{~nm}$; deceasing the gap between the QDs and the source/drain/gate; and by decreasing the size of the electrodes.

\subsubsection{Doping the Substrate with the FIB}

Since the photolithography-based SET architecture was not producing working SETs, was eliminated. To reduce variables associated with the photolithography, the SETs architecture was etched with the FIB and each step to fabricate the devices remained as described earlier. 
To fabricate an etch-based device without photolithography, the SET architecture, (i.e. pads and electrodes) must be isolated from the gold coated substrate, accomplished by FIB etching. The initial hypothesis was that the longer the etch time the better the isolation. However, this was not correct. With a longer etch time for a given area the resistance decreased between the etched-out feature and the substrate.

The experiment began by using the fabrication mode of the FIB, because others had used this mode and had established the beam parameters to etch $\mathrm{Cr}$. The etched pads were then characterized with the 4200 SPA. The 4200 SPA was used to measure the I-V with two probes, one on the perimeter etched square and the other on the substrate to check if they were isolated. As shown in Figure 90 - Figure 92 the etched perimeters of the square were not isolated, the graphs actually demonstrated that the resistance was decreasing with longer total etching times for a given area. In other words the etched perimeter around the gold coated square pad was not isolated from the gold coated substrate.

Since the longer etch times were not isolating the perimeter of the gold coated square pad from the gold coated substrate, the total etch time was decreased and the beam remained the same, M1-500. Also instead of using fabrication mode, vector scan mode was used because it was easier to control the total etch time. An experiment was conducted with three different etch times. As shown in Figure 93 - Figure 95 the pads were isolated from the substrate in as little as 15 seconds.

This result shows that the total etching time was critical to fabricating the etch-based SET architecture. The thin film of $\mathrm{Cr} / \mathrm{Au}$ does not take a long time to etch with the FIB. 


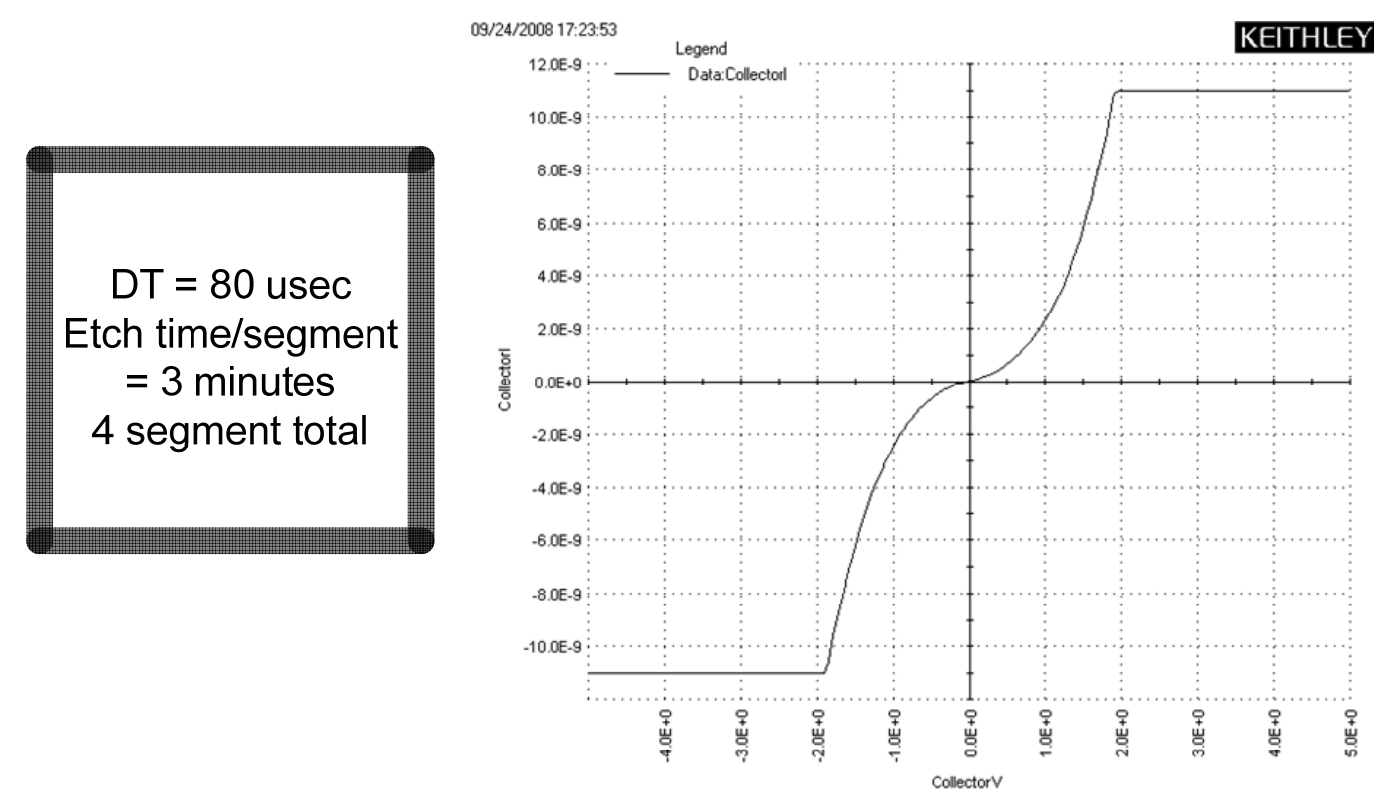

Figure 90. FIB etched perimeter is indicated by the gray lines in the shape of a square, using beam M1-500 in Fabrication Mode. The actual line width is $5 \mu \mathrm{m}$ and the size of the square is about $100 \mu \mathrm{m}$ by $100 \mu \mathrm{m}$. The total etch time $\sim 12$ minutes.
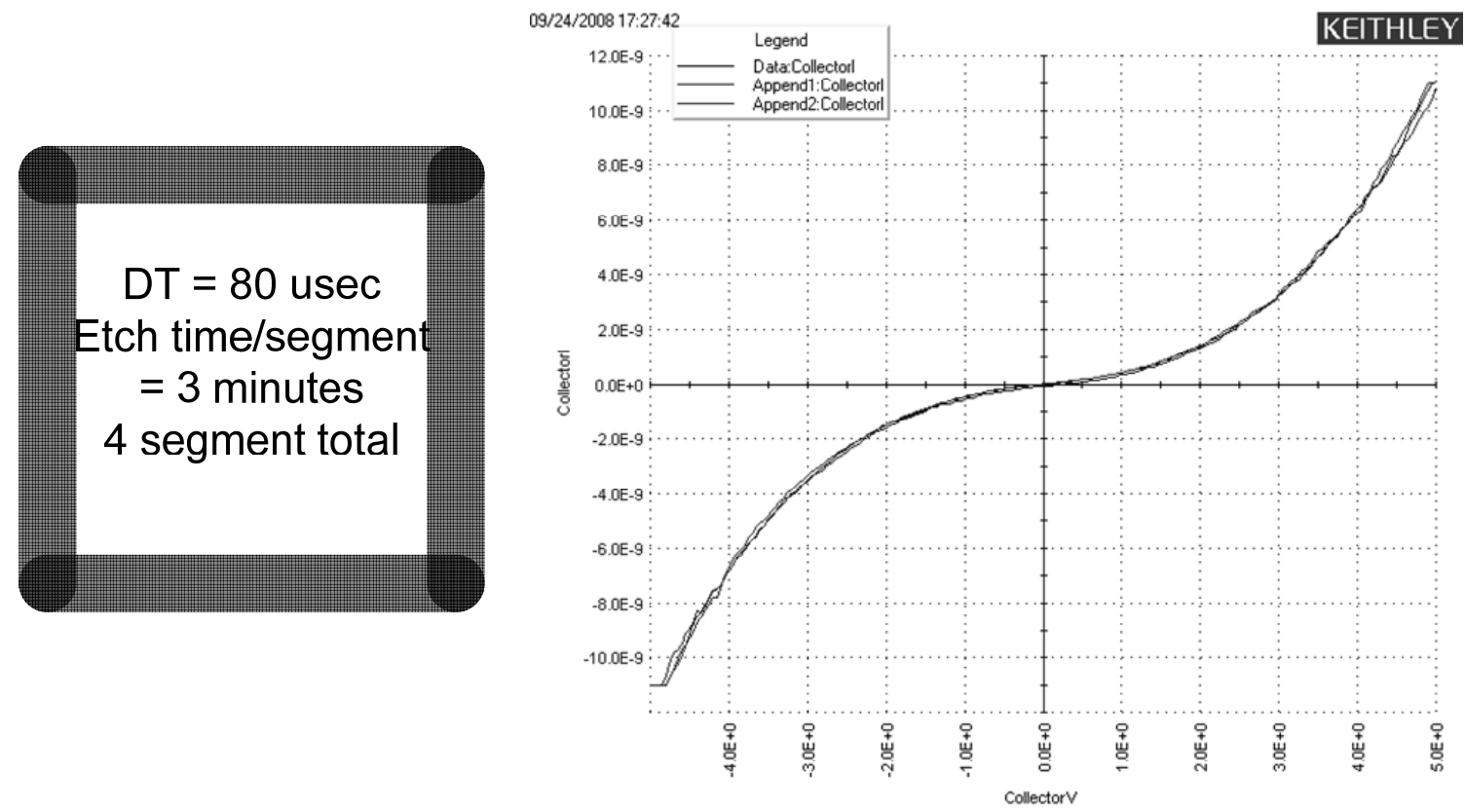

Figure 91. FIB etched perimeter is indicated by the gray lines in the shape of a square, using beam M1-500 in Fabrication Mode. The actual line width is $10 \mu \mathrm{m}$ and the size of the square is about $100 \mu \mathrm{m}$ by $100 \mu \mathrm{m}$. Total etch time $\sim 12$ minutes. 

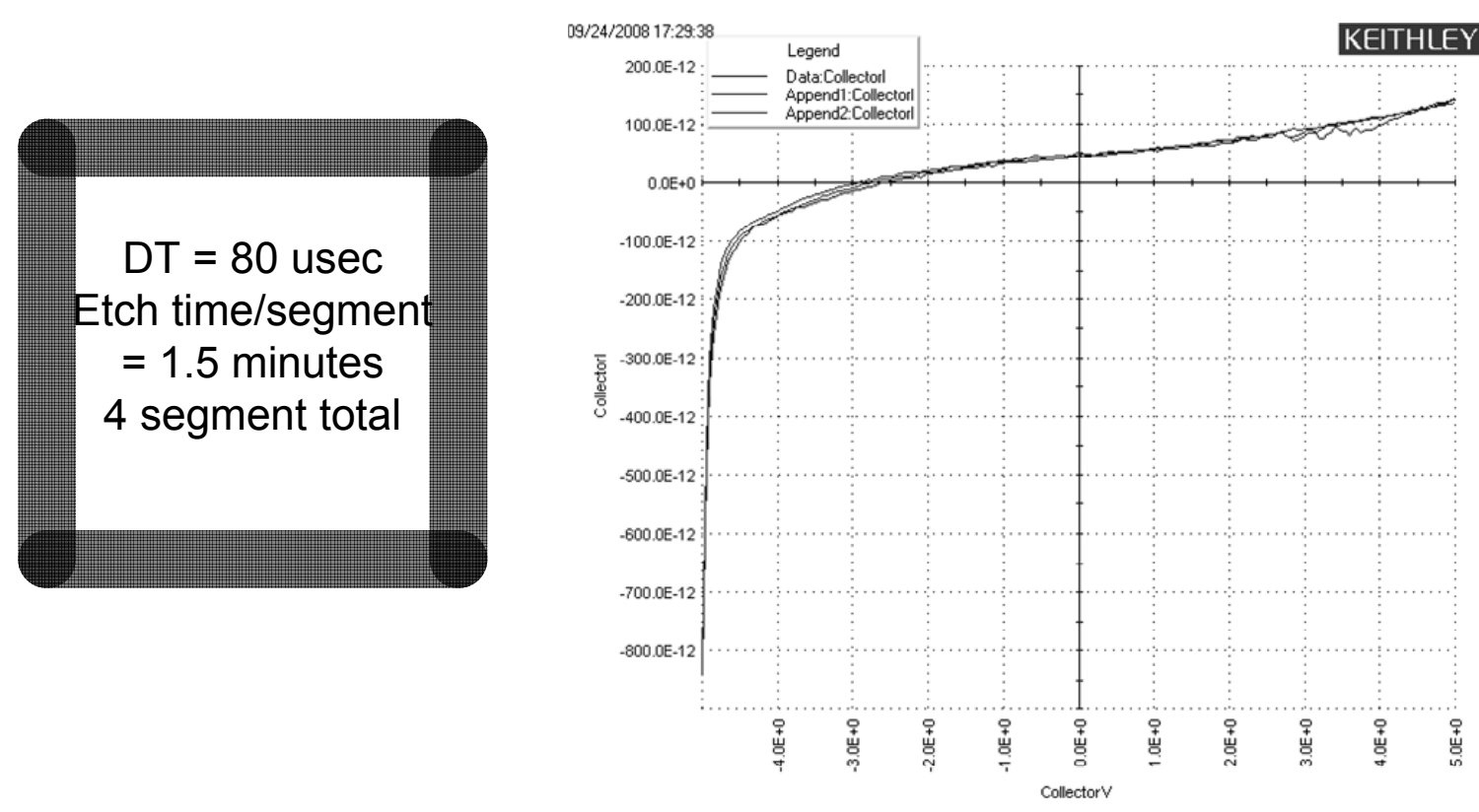

Figure 92. FIB etched perimeter is indicated by the gray lines in the shape of a square, using beam M1-500 in Fabrication Mode. The actual line width is $10 \mu \mathrm{m}$ and the size of the square is about $100 \mu \mathrm{m}$ by $100 \mu \mathrm{m}$. Total etch time $\sim 6$ minutes.
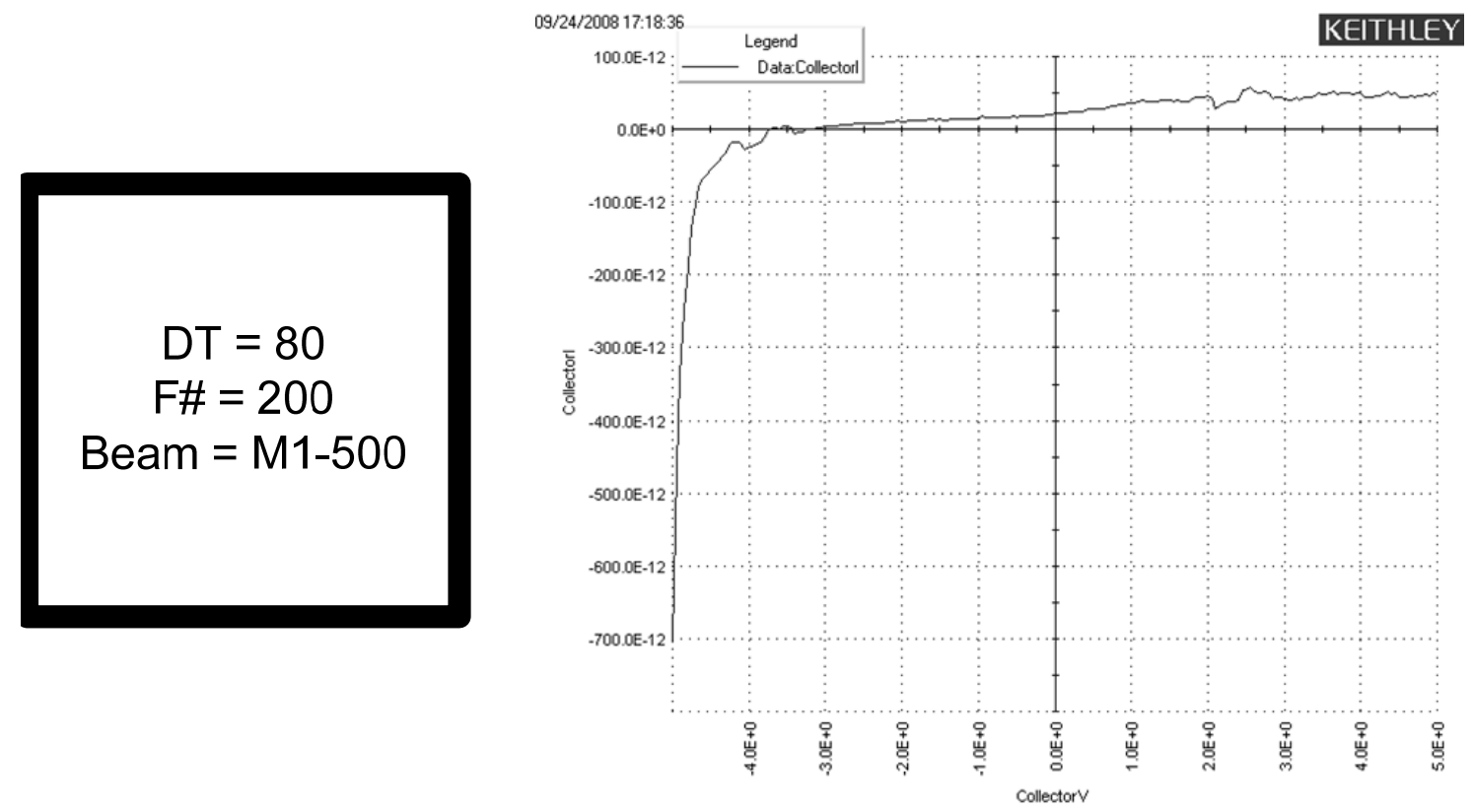

Figure 93. FIB etched perimeter is indicated by the black lines in the shape of a square, using beam M1-500 in Vector Scan Mode. The actual line width is $5 \mu \mathrm{m}$ and the size of the square is about $100 \mu \mathrm{m}$ by $100 \mu \mathrm{m}$. Total etch time $\sim 1.5$ minute. 

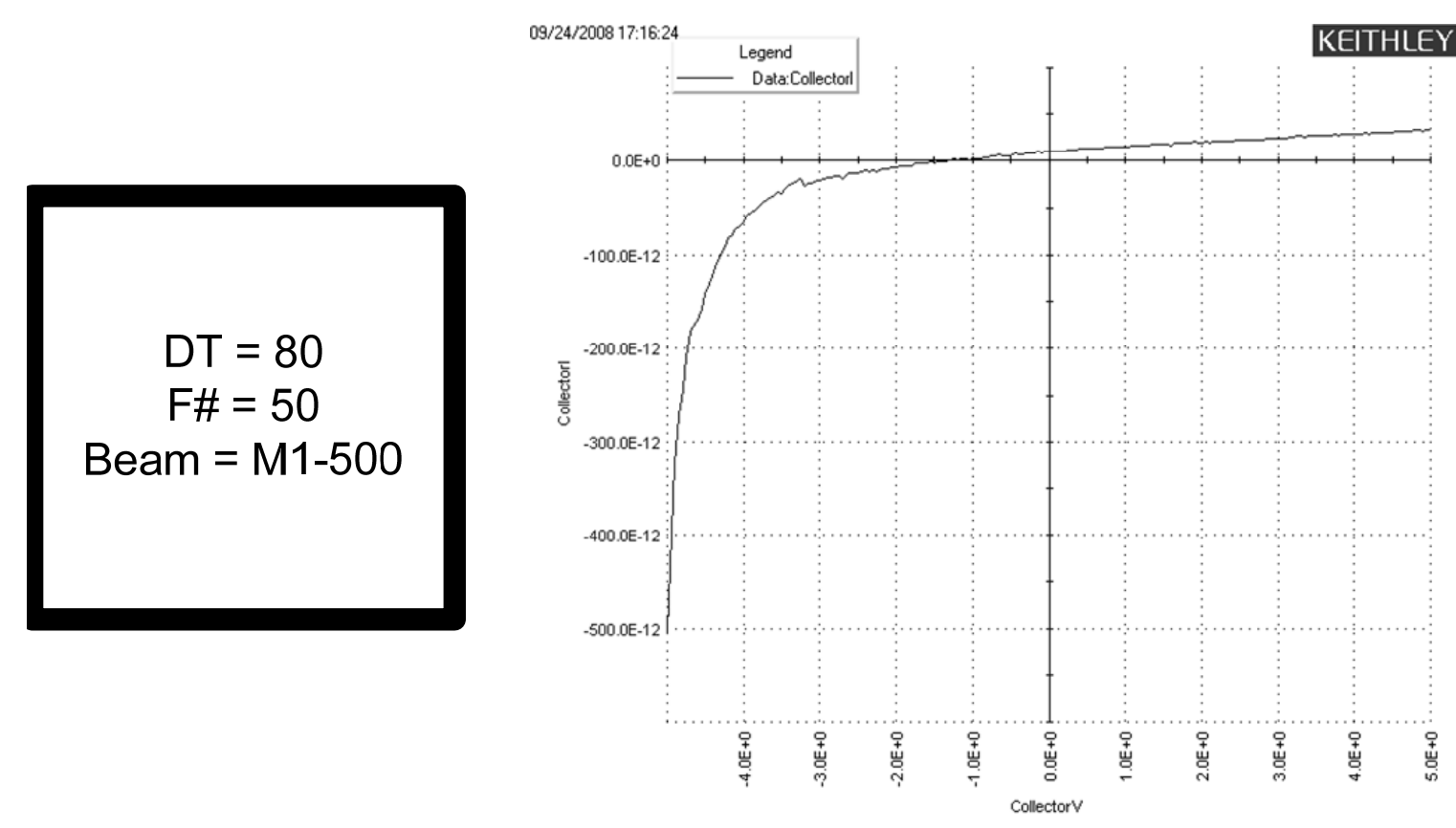

Figure 94. FIB etched perimeter is indicated by the black lines in the shape of a square, using beam M1-500 in Vector Scan Mode. The actual line width is $5 \mu \mathrm{m}$ and the size of the square is about $100 \mu \mathrm{m}$ by $100 \mu \mathrm{m}$. Total etch time $\sim 30$ seconds.

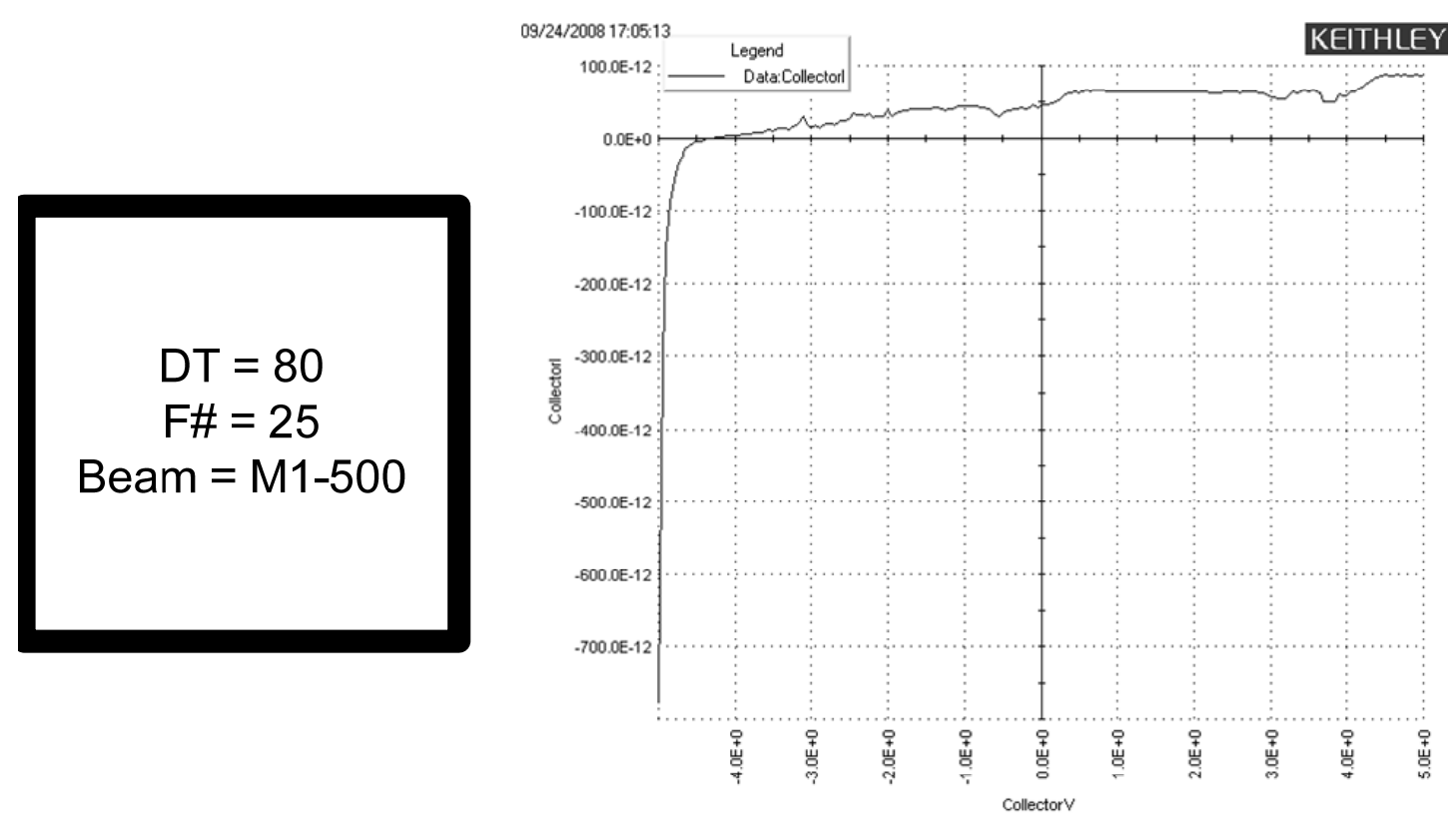

Figure 95. FIB etched perimeter is indicated by the black lines in the shape of a square, using beam M1-500 in Vector Scan Mode. The actual line width is $5 \mu \mathrm{m}$ and the size of the square is about $100 \mu \mathrm{m}$ by $100 \mu \mathrm{m}$. Total etch time $\sim 15$ seconds. 


\subsubsection{FIB Deposited Tungsten QD Characterization}

The next feature of the SET investigated was the FIB deposited tungsten QDs. The QDs and electrodes are the only active features of the SET and are the most important components of SETs. FIB QDs were formed based on a combination of etching and deposition during the deposition of the tungsten [93]. The parameters that can be controlled directly from the on-broad software were the: interlace, dwell time, deposition area, and ion beam current. There are a variety of other factors that influence the deposition as described earlier but this experiment was focused on the parameters that can be controlled through the on-broad software.

After analyzing the recipe that Karre [86] used to deposit the QDs and comparing that to the current settings of the FIB, it was determined that the deposition area, interlace (8), dwell time $(9 \mu \mathrm{sec})$, and beam selection (M1-200) were all the same but there was one major difference. The ion beam current of beam M1-200 had almost doubled in value from $2.33 \mathrm{nA}$ to $4.01 \mathrm{nA}$. The change in beam current most likely caused a change in the QD size and distribution. To confirm this the QDs were deposited with the FIB and imaged with the with the FESEM.

From Figure 96 it is clear that the QDs produced with the same recipe that Karre [86] developed were no longer producing the same density and size of QDs as shown in Figure 97. The majority of the QDs appeared to be less then $10 \mathrm{~nm}$ in diameter but they were separated by more then $10 \mathrm{~nm}$. To produce QDs less than $10 \mathrm{~nm}$ in diameter and separated by a couple of nanometers a new deposition recipe had to be created. 


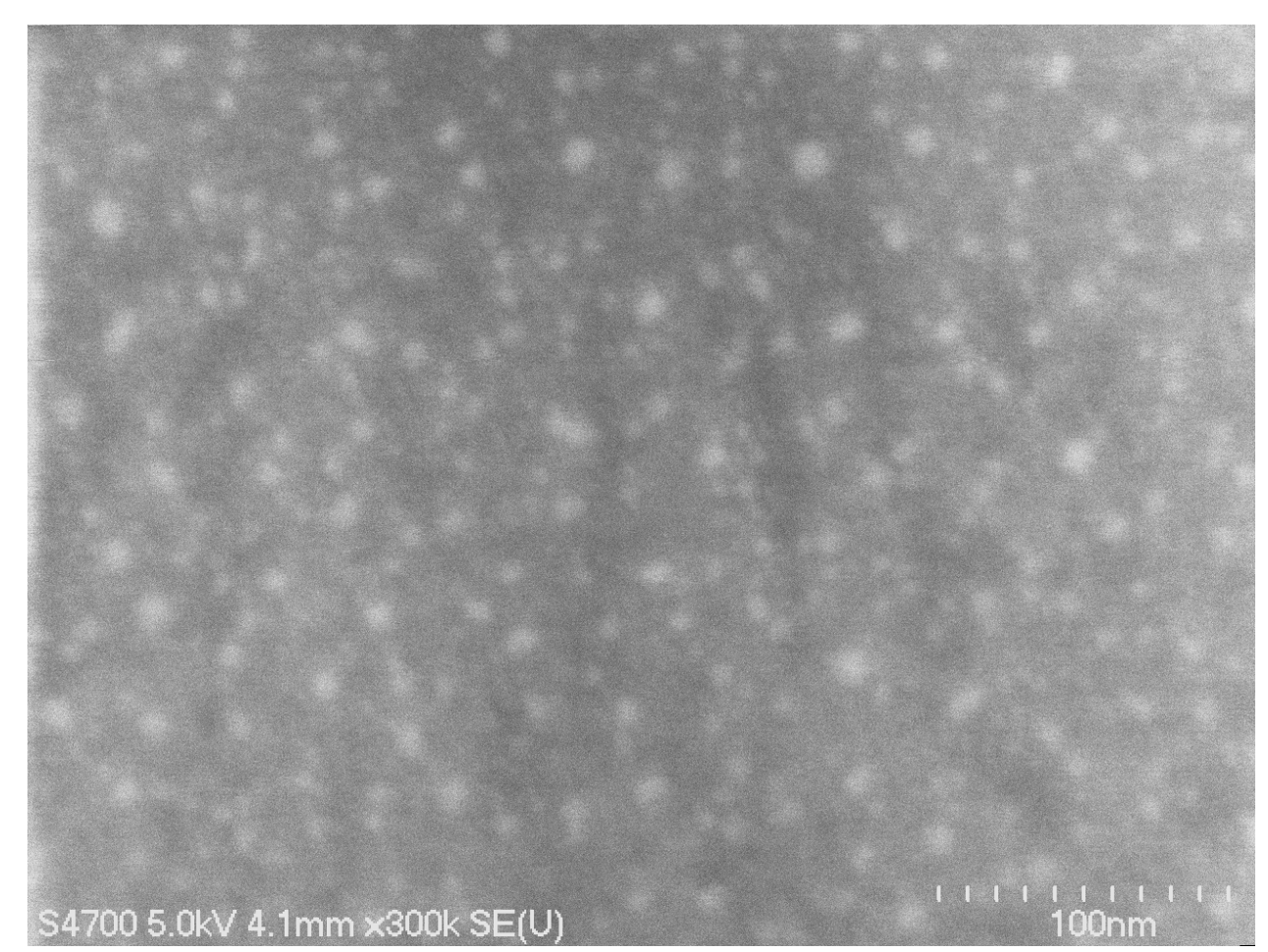

Figure 96. FIB deposited QDs, using beam M1-200 with an interlace of 8, and Dwell time of $9 \mu \mathrm{sec}$, beam current of $0.401 \mathrm{nA}$, and a depositon area of the $16.062 \mu \mathrm{m}^{2}$.

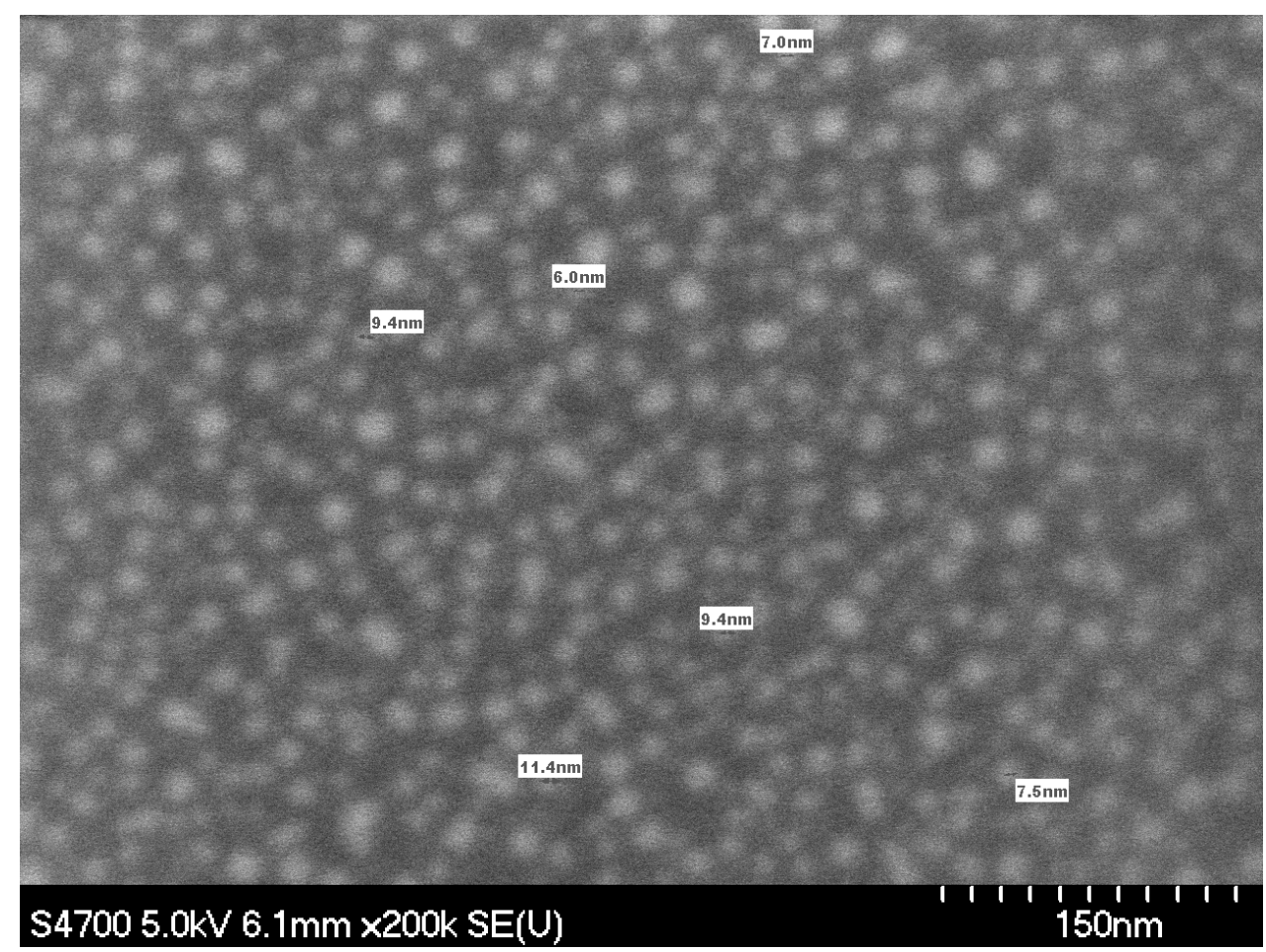

Figure 97. FIB deposited recipe for QDs, using beam M1-200 with an interlace of 8, and Dwell time of $9 \mu \mathrm{sec}$, beam current of $2.33 \mathrm{nA}$, and a depositon area of the $16.062 \mu \mathrm{m}^{2}$. 
Since the ion beam current had changed significantly since Karre [86] had deposited QDs, the FIB had to be re-characterized to find the optimal settings. A systematic approach was used to find a recipe that would produce QDs less than $10 \mathrm{~nm}$ in diameter and separated by a couple of nanometers. The experiment was executed with the same sized pattern area $(16.062 \times 16.062 \mu \mathrm{m})$, but the dwell time was varied from 3 to $11 \mu \mathrm{sec}$, two different interlace values 4 and 8, and two different ion beams M1-100 and M1-200. During the deposition, beam M1-100 and M1-200 had beam currents of 0.893 and 4.01 $\mathrm{nA}$. The QDs were analyzed at two magnifications $110 \mathrm{k}$ and $300 \mathrm{k}$. To image the tungsten dots the parameters were: accelerating voltage $=5 \mathrm{keV}$, emission current $=10$ $\mathrm{nA}$, working distance $=4.1 \mathrm{~mm}$, mode $=$ ultra high resolution, condenser len $=4-12$, and the upper detector. FESEM analyses of beams M1-200 and M1-100 are shown in Figure 98 - Figure 101. There did not appear a clear trend for tungsten dot size versus dwell time as Karre [86] noticed. There does appear to be a pattern for both beams and interlaces at dwell times of 4 and $11 \mu \mathrm{sec}$ the QDs are the largest in the range of dwell times used. Beam M1-200, as shown in Figure 98 - Figure 99 there does not appear to be a good recipe to produce the $10 \mathrm{~nm}$ QD with a spacing of $\sim 2-5 \mathrm{~nm}$ separation. With beam M1-200 having an ion beam current of $4.01 \mathrm{nA}$, it should not be used to make tungsten QDs for the SETs unless the ion beam current changes.

FESEM images of the QDs deposited using beam M1-100 are shown in Figure 100 Figure 101 there appears to be a few different good recipes that were producing QDs less than $10 \mathrm{~nm}$ and $2-5 \mathrm{~nm}$ separation. For most of the images the dots appear to be separated by $2-5 \mathrm{~nm}$ but it was difficult to determine if they were touching. Using beam M1-100 with an interlace of 4, and dwell times of 8,9 , and $10 \mu \mathrm{sec}$, were selected to make the tungsten QDs for the SETs. After the QD deposition, they were oxidized and passivated as previously described. 


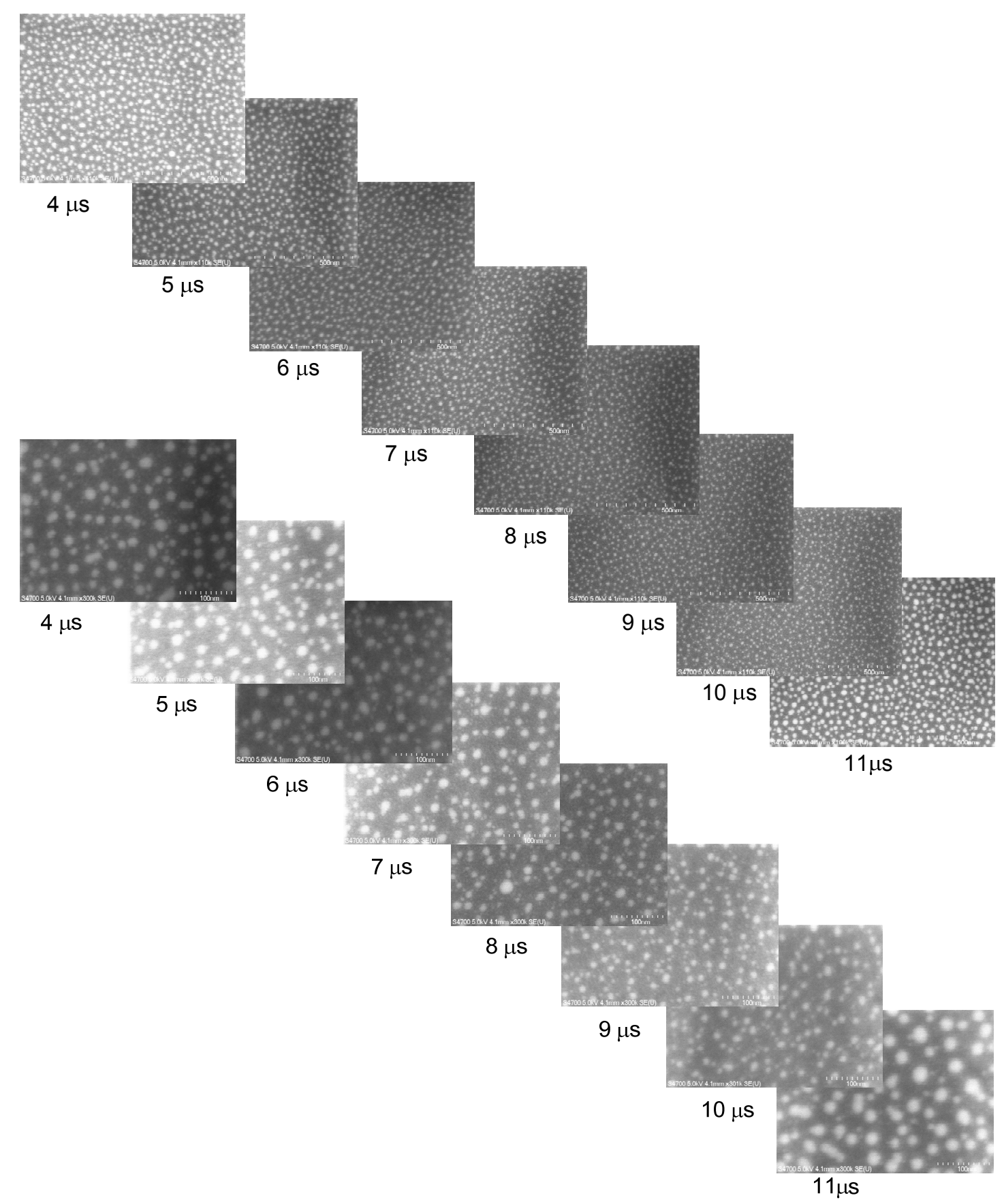

Figure 98. Imaged at 110k (top) and 300k (bottom) with the FESEM image of tungsten QDs deposited using beam M1-200 with a beam current of $4.01 \mathrm{nA}$, interlace 4 and a dwell time varied from $4 \mu$ s to $11 \mu \mathrm{s}$. 


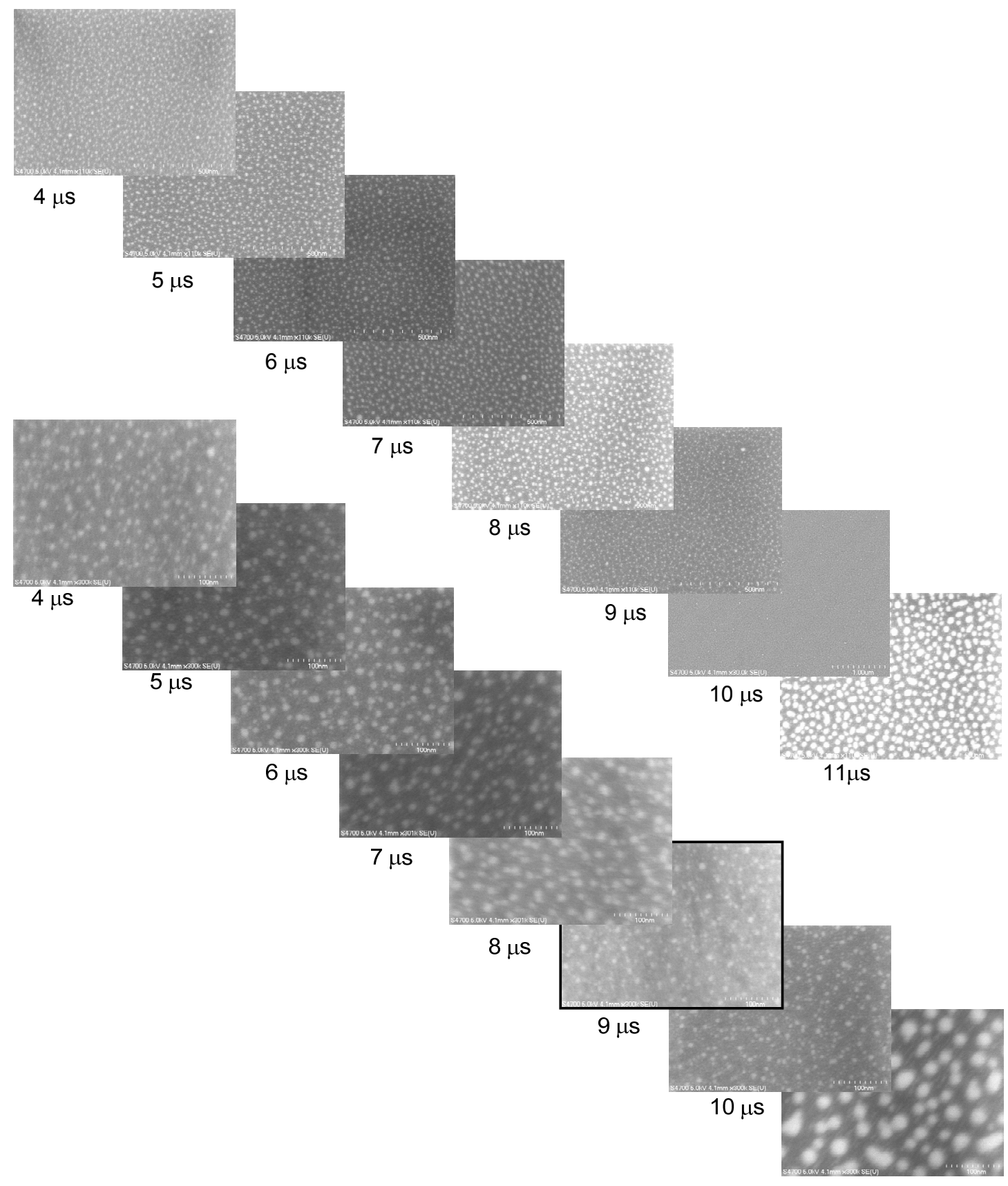

$11 \mu \mathrm{s}$

Figure 99. Imaged at 110k (top) and 300k (bottom) with the FESEM, image of tungsten QDs deposited using beam M1-200 with a beam current of $4.01 \mathrm{nA}$, interlace 8 and a dwell time varied from $3 \mu$ s to $11 \mu \mathrm{s}$. 


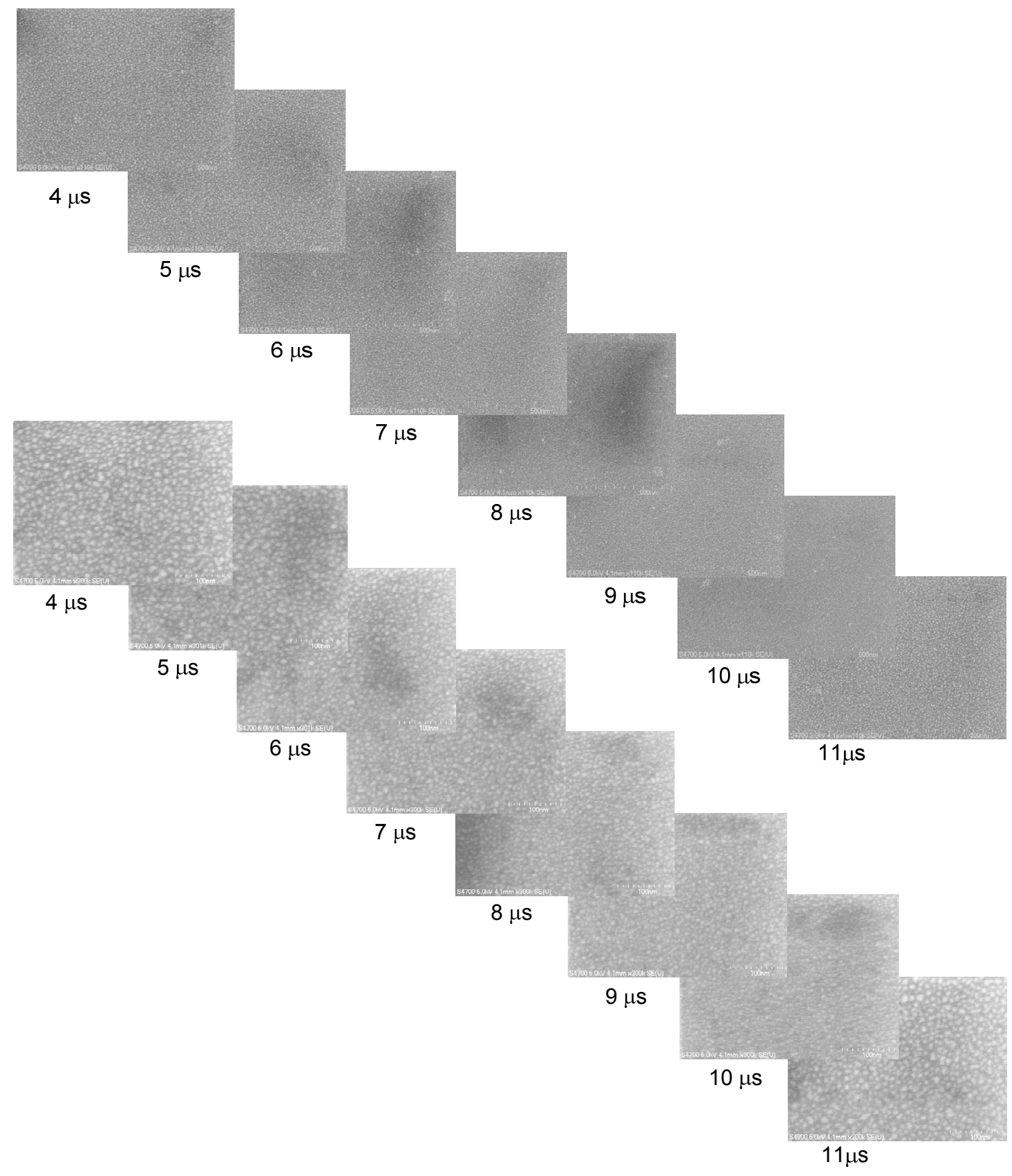

Figure 100. Imaged at 110k with the FESEM, image of tungsten QDs deposited using beam M1-100 with a beam current of $0.893 \mathrm{nA}$, interlace 4 and a dwell time varied from $4 \mu \mathrm{s}$ to $11 \mu \mathrm{s}$. 


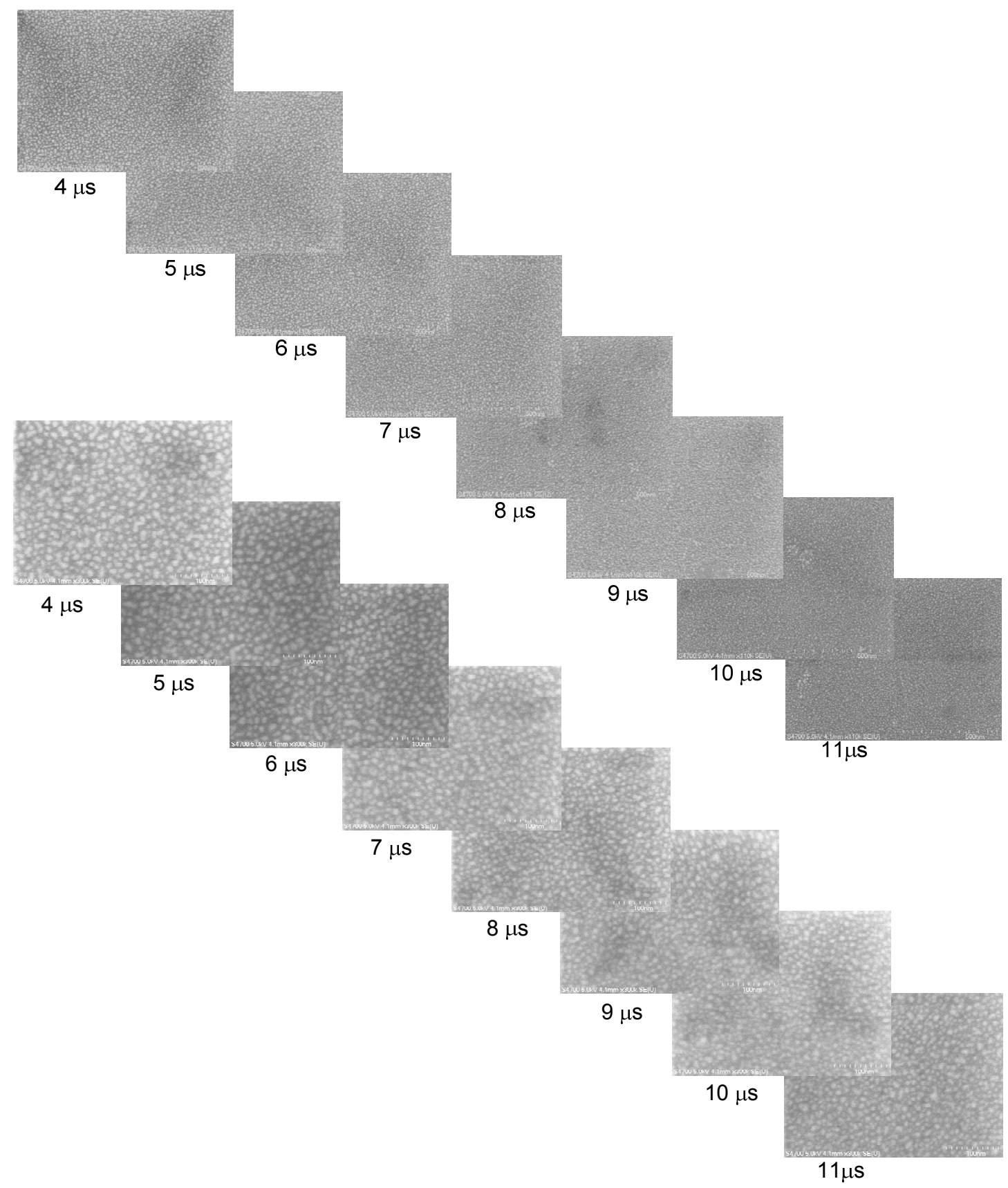

Figure 101. Imaged at 110k (top) and 300k (bottom) with the FESEM, image of tungsten QDs deposited using beam M1-100 with a beam current of $0.893 \mathrm{nA}$, interlace 8 and a dwell time varied from $4 \mu$ s to $11 \mu \mathrm{s}$. 


\subsubsection{Cutting the Gap}

As mentioned earlier the overall device capacitance can be reduced; by decreasing the QD to $<10 \mathrm{~nm}$; deceasing the gap between the QDs and the source/drain/gate; and by decreasing the size of the electrodes. As shown in the previous section a few different recipes were found that will produce QDs $<10 \mathrm{~nm}$ in diameter, the next component to look at was the gap separating the source/drain/gate. The separation gap between the source/drain along with the QDs density and size determines the overall capacitance of the device, which determines if the device will function at room temperature (threshold temperature) $[94,95]$. According to [95] the larger the separation gap (limited to 100 $\mathrm{nm}$ ) the threshold voltage will be larger, hence I-V characteristics will display a larger Coulomb blockade region.

The separation gap between the source/drain/gate was fabricated using beam M0-20 at various magnifications. The initial hypothesis was that a $20-100 \mathrm{~nm}$ separation gap could only be fabricated using the largest magnification and smaller window area. But this turned out to be incorrect. As shown in Figure 102 a $20 \mathrm{~nm}$ gap was fabricated with a window area of $32 \mu \mathrm{m}^{2}$ and a zoom of 8 with beam M0-20 the image taken after the QDs were deposited. The electrodes appear to be slightly deteriorated after the deposition of the QDs. With a window area of $4 \mu \mathrm{m}^{2}$ and a zoom of 2 with beam M0-20 the as shown in Figure 103 the electrodes were deteriorated during the QD deposition. This was probably because the electrodes were weakened during the imaging to cut the gap with the FIB. 


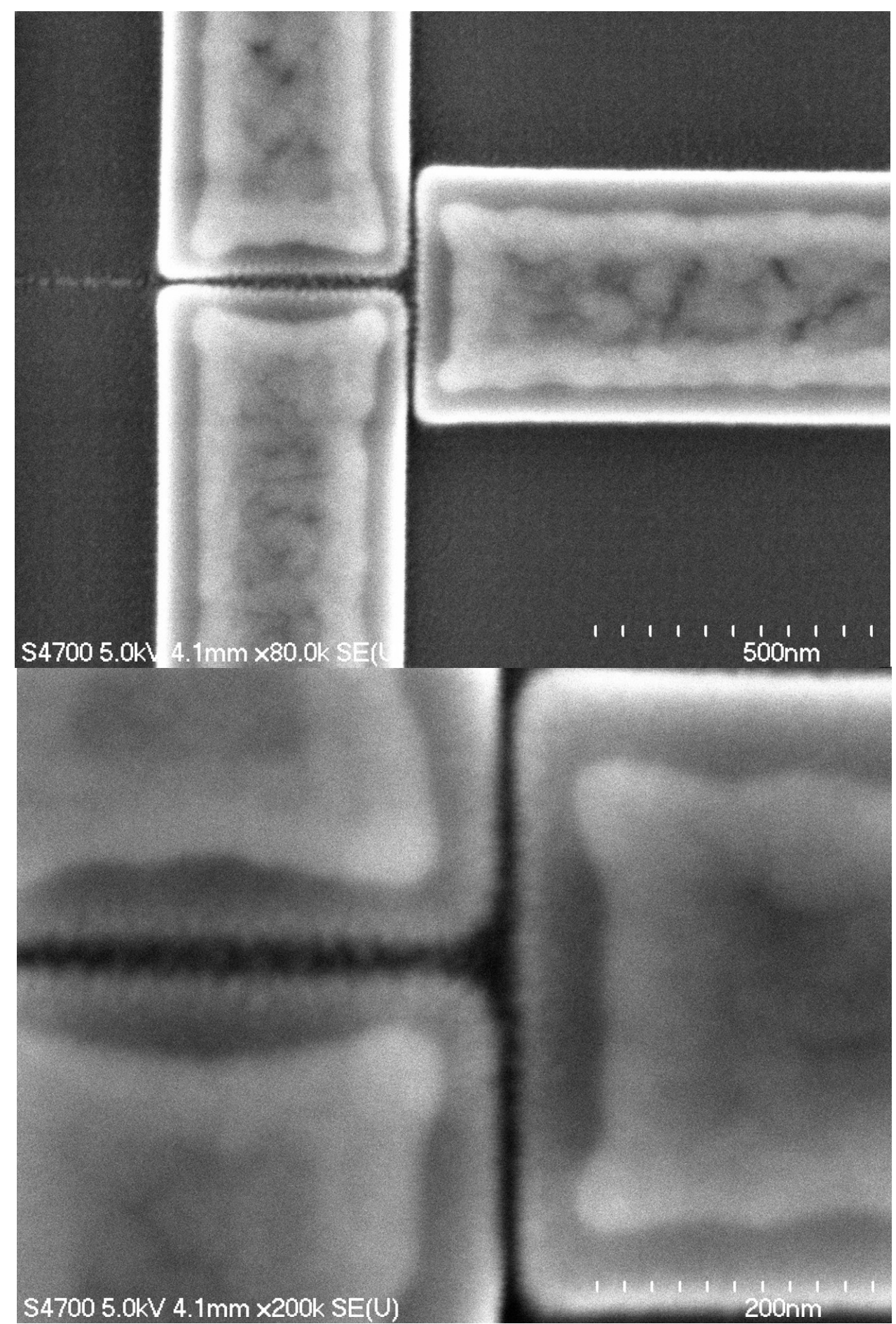

Figure 102. Gap etched using M0-20 with an area of 32 and zoom of 8 . The QDs were deposited using beam M1-100 with an interlace of 4 and dwell time of $8 \mu \mathrm{sec}$. 


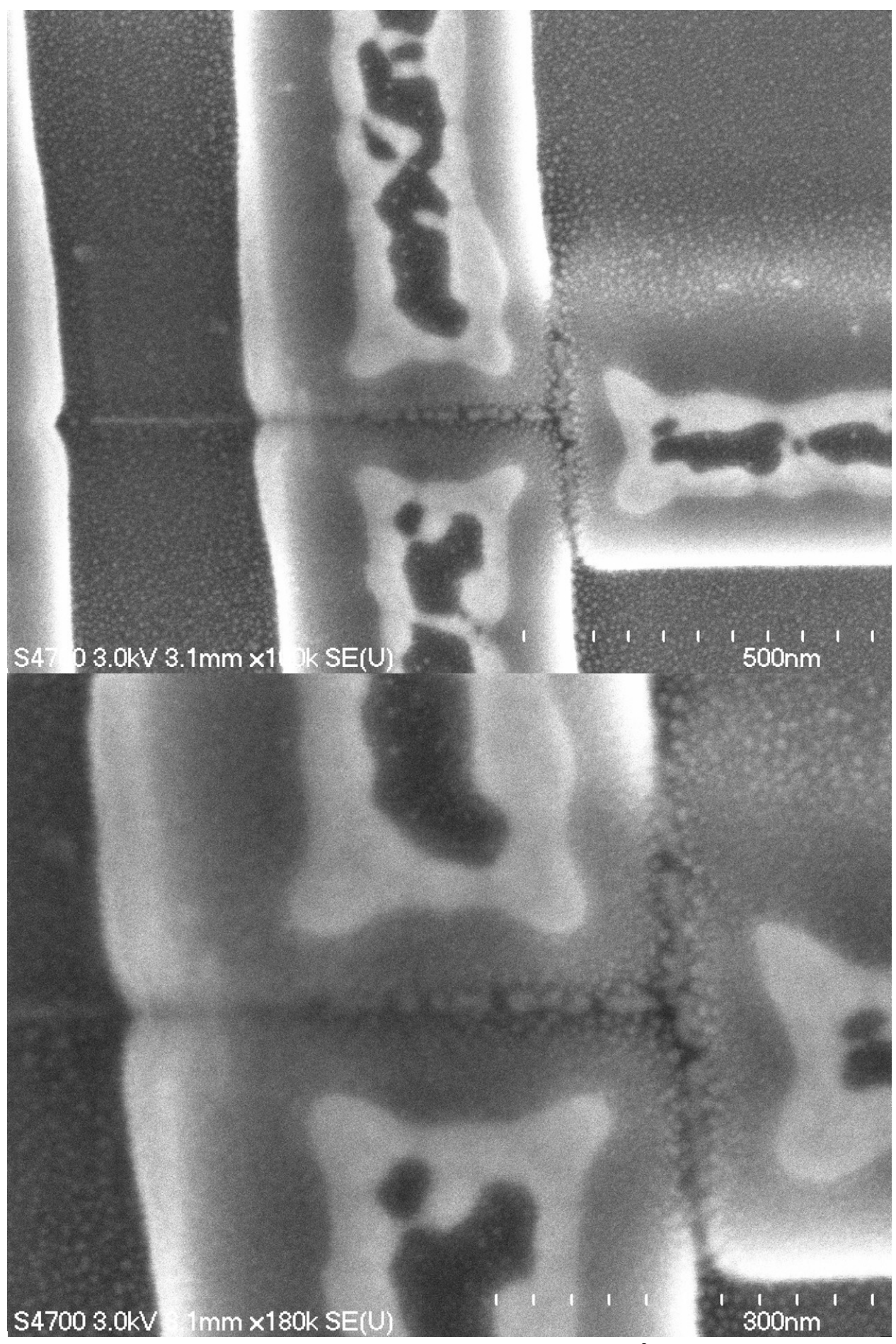

Figure 103. Gap etched using M0-20 with an area of $4 \mu \mathrm{m}^{2}$ and zoom of 2. The QDs were deposited using beam M1-100 with an interlace of 4 and dwell time of $8 \mu \mathrm{sec}$. 
But after fabricating 15 SETs with the new gap cutting method and QD deposition recipes, the SET still did not function. Everything else was unchanged from forming the tunnel junction by oxidizing the QDs in peracetic acid for two minutes, and then passivating the devices with $\mathrm{Al}_{2} \mathrm{O}_{3}$ just as described by $[86,87]$

Since functional SETs have not been achieved reliably, in this work, a final fabrication process for the bR-SET cannot be outlined. Other parameters not yet understood are most likely causing the limited repeatability of the SETs functionality or possibly related to inconsistency with the ion current of the FIB. 


\section{Chapter 8 Model Results}

This section will present an empirical model for the bR-SET and initial results on the development of technology for a sensing platform which incorporates a single electron transistor with bR.

\subsection{Schematic bR-SET Model}

As mentioned earlier, $\mathrm{bR}$ is a photon activated bionanomaterial which generates a voltage with visible light. So, one possible method of incorporating bR and a SET is to link them at the gate of the SET. By linking them at the gate a change in the voltage potential of $\mathrm{bR}$, by modulating the light intensity, would be detected by a change in the source-drain current. As described earlier bR can be modeled as a resistor and capacitor in parallel and knowing a few physical parameters of the deposited film the photovoltaic response can be estimated as describe earlier. A possible representative schematic model of the combined bR-SET, bionanohybrid device is shown in Figure 104 and is based on the schematic model of the orthodox model [96] of a SET and the schematic model of bR. Both of these components have individual theoretical and/or empirical models but no one has integrated them.

\section{Model SET Model bR $\quad$ Model bR/SET}

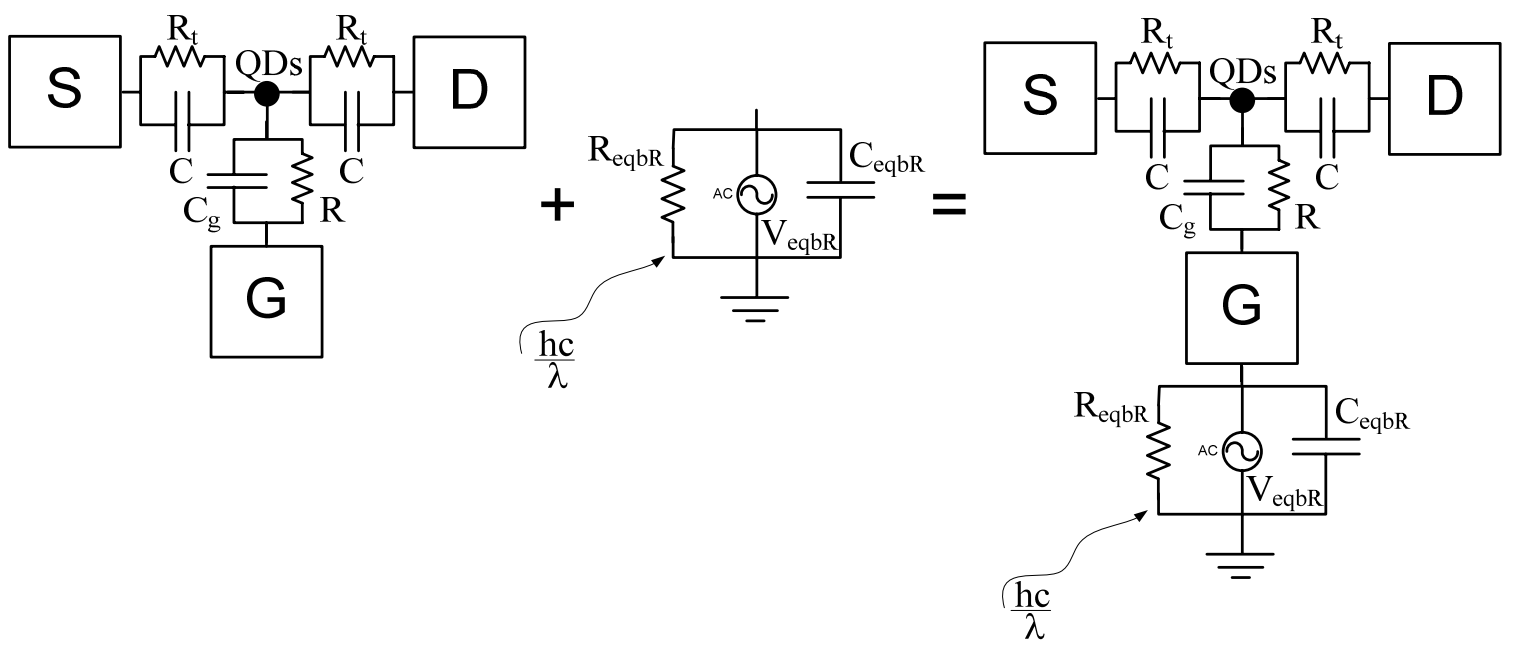

Figure 104. Representative schematic of a SET with bR integrated on the gate.

Initial analysis of incorporating a SET and $b R$ will be examined in this section. As described earlier room temperature SETs are extremely sensitive to changes in capacitance, in fact they will loose their room temperature functionality if the capacitance 
is too large. So, it is important to determine if integrating bR on the gate of the SET will alter the capacitance of the SET.

The overall capacitance of a SET can be calculate as Karre [86] showed and estimated to be about $0.5 \mathrm{aF}$. Based on the bR capacitance measurements and analysis presented earlier, the relative permittivity was estimated to be $\sim 2.2$, and knowing the area and \# of layers deposited an estimate of the capacitance can be calculated. However, for this theoretical analysis the value of the capacitance shown in Figure 58 is used, $22 \mathrm{pF}$.

Theoretically if the bR is placed on the gate the SET the capacitance of the bR is in series with the gate capacitance. Mathematically this can be represented as two capacitors in series as shown in Eq. (7). From the calculation it is clear that the capacitance of the bR has no significant effect on the overall device capacitance, which suggests that integrating $b R$ on the gate of the SET, will have no effect on the functionality of the SET. So the next step is to attempt to integrate bR on the gate of an SET.

$$
\frac{1}{\mathrm{C}^{*}}=\frac{1}{\mathrm{C}_{\mathrm{g}}}+\frac{1}{\mathrm{C}_{\mathrm{br}}}=\frac{1}{0.5 \mathrm{aF}}+\frac{1}{22 \mathrm{pF}} \stackrel{\mathrm{C}^{*}}{\longrightarrow} 0.5 \mathrm{aF}
$$

\subsection{Activation of bR-SET Without Sputtered Gold Electrode}

The next sections present initial results on the development of technology for a sensing platform which incorporates single electron transistors with bR. SETs were fabricated by a combination sputter deposition and FIB etching as described earlier. The bR was deposited on the gate of the SET by electrophoretic deposition as described earlier. A graphical representation of the two integrated is shown in Figure 105. Tests were conducted using the Keithley 4200 SPA with a preamp connected to the collector. The test parameters for the data collected in I-V curves (Figure 106) were; mode voltage sweep $+/-2 \mathrm{~V}$; step $0.5 \mathrm{~V}$; fast speed; preamp connected to the collector. The test parameters for the current-time data (Figure 107 - Figure 111) were; sample mode; drain biased to $0.5 \mathrm{~V}$; compliance current of $1 \times 10^{-10} \mathrm{Amp}$; range on auto; source measurement unit (SMU) 1; preamp connected to collector; SMU 2 connected to drain; gate was floating with the bR.

First, the SETs were characterized without bR deposited on the gate, to see if light would alter the functionality. As shown in Figure 106 and Figure 107 there was no significant difference in the functionality of the SET with and without light. This indicated that photons from the light source did not influence the functionality of the SET, suggesting that any modulation in the functionality of the bR-SET when light activated can be attributed to the photovoltaic characteristics of $b R$.

Next, tests were conducted with bR immobilized on the gate of the SETs. The effects of integrating bR on the SET is shown in Figure 108 - Figure 111. These figures show that 
the light activated bR immobilized on the gate modulates the functionality of the SET, measured in sample mode. Figure 108 - Figure 111 show that flashing the bR-SET with light causes a sudden change in the source-drain current and when no light is flashing there is no change in the current with respect to time. Figure 108 - Figure 111 show that changing the flash rate does not affect the magnitude of the response but that it does follow the flash rate of the light. More tests should be conducted to quantify interactions between the bR and SET and to understand how the SPA is collecting data during the experiment.

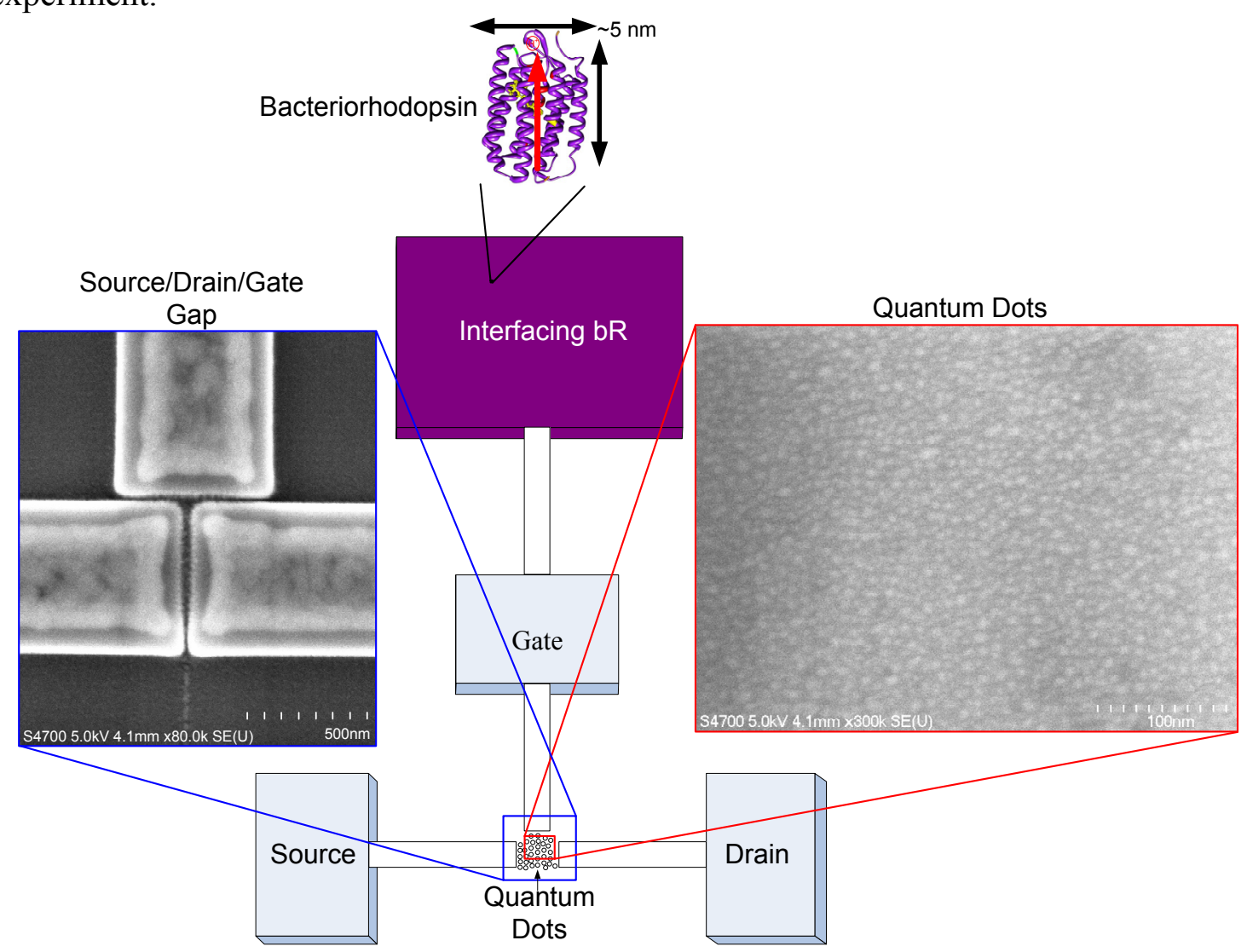

Figure 105. Sensing platform, bR interfaced with a SET. 


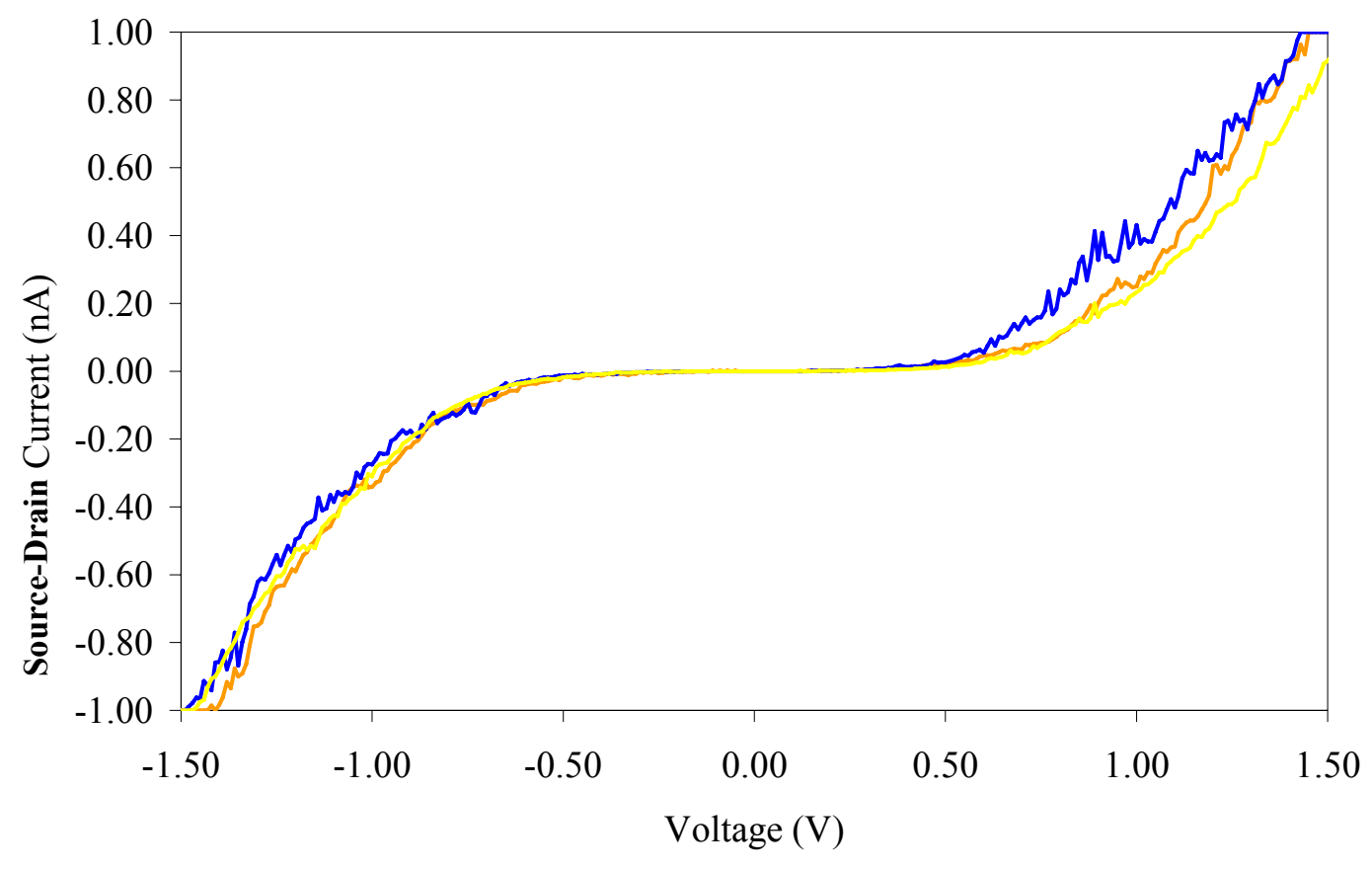

— With light flashing — Without light — With constant light

Figure 106. Current versus voltage measurements of a SET without bR immobilized on the gate with and without light.

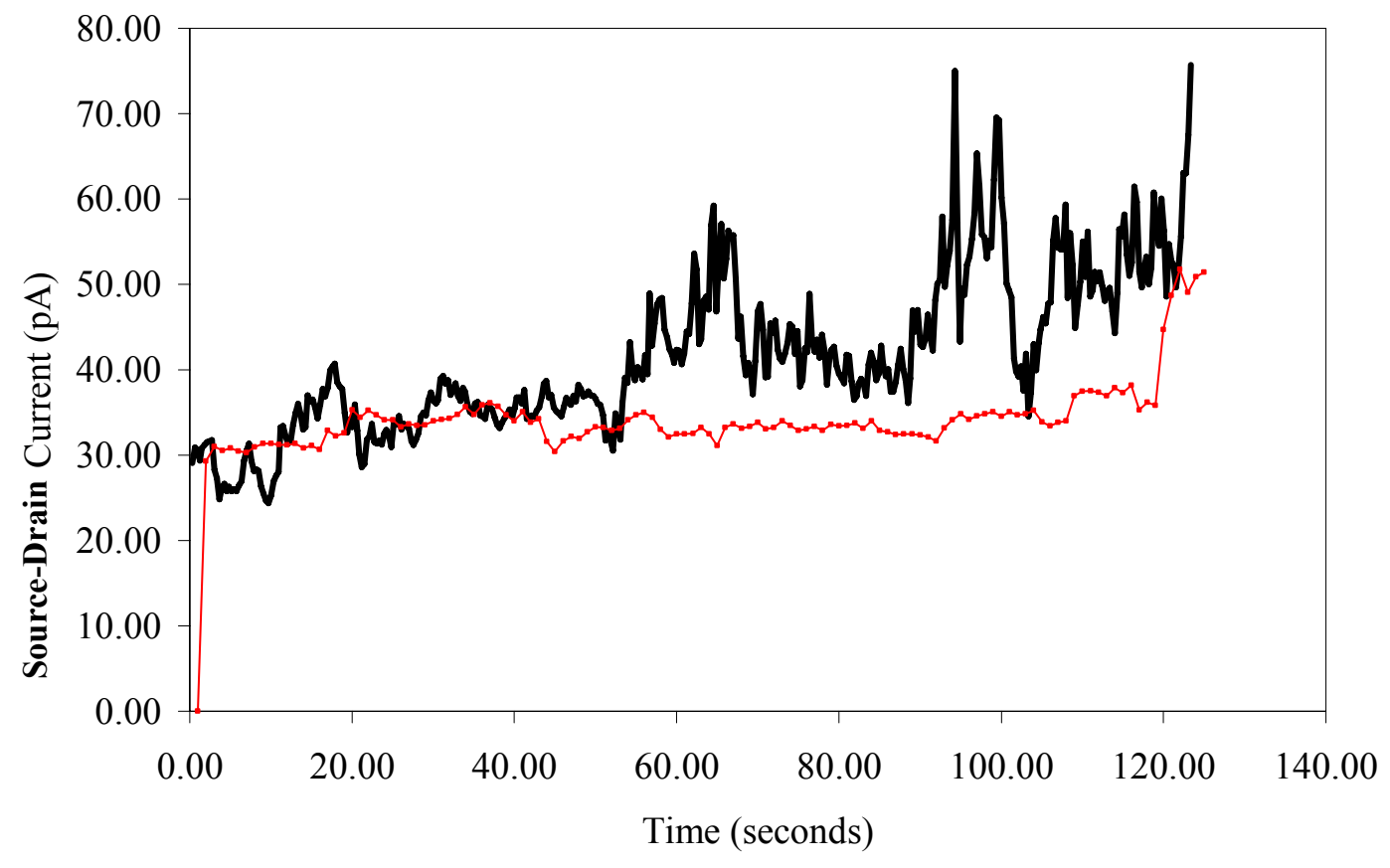

- Without light $\rightarrow$ With light flashing

Figure 107. Current versus time measurements of a SET without $b R$ interfaced with the gate with and without light.

106 


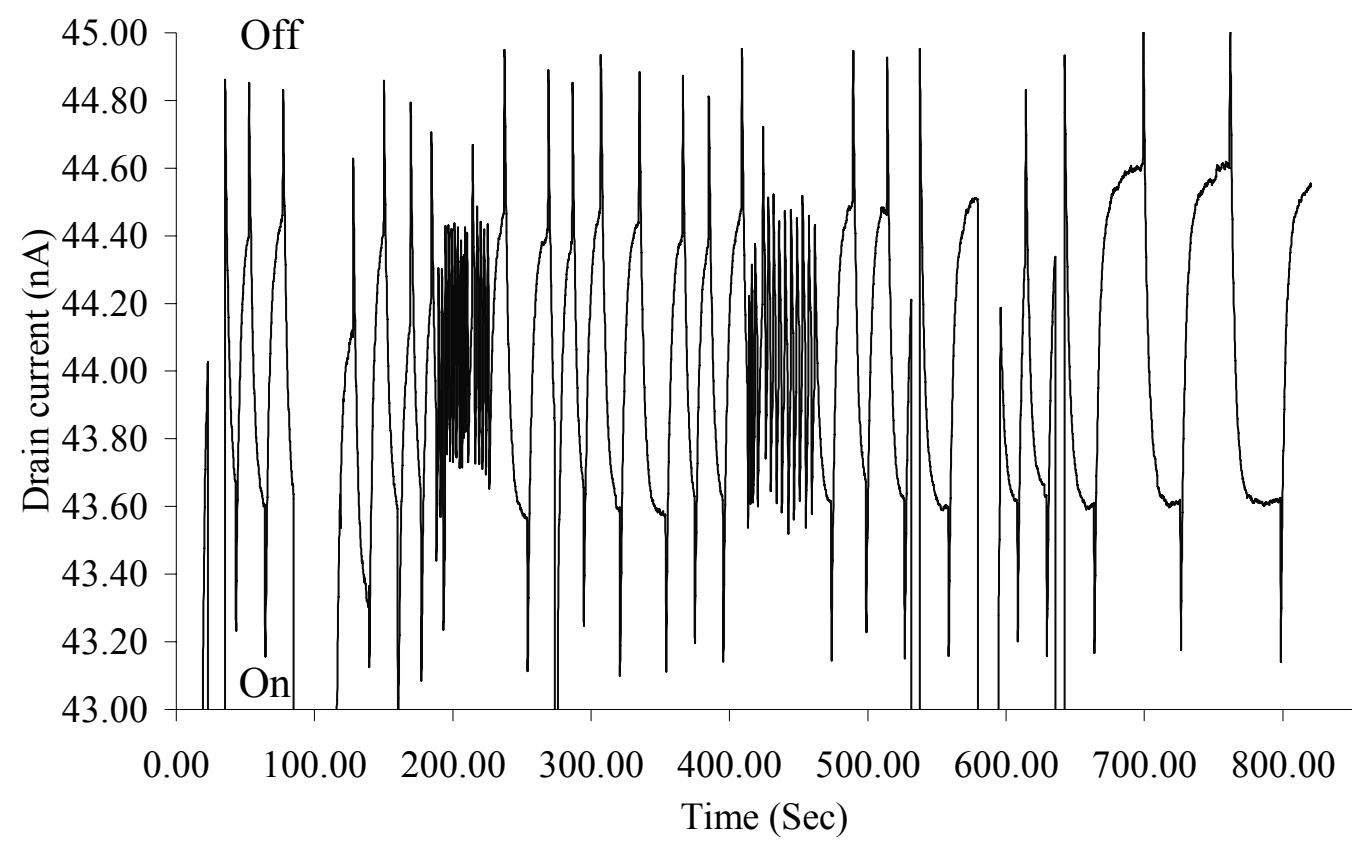

Figure 108. Current versus time measurements of a SET with bR interfaced with the gate and flashed at various rates.

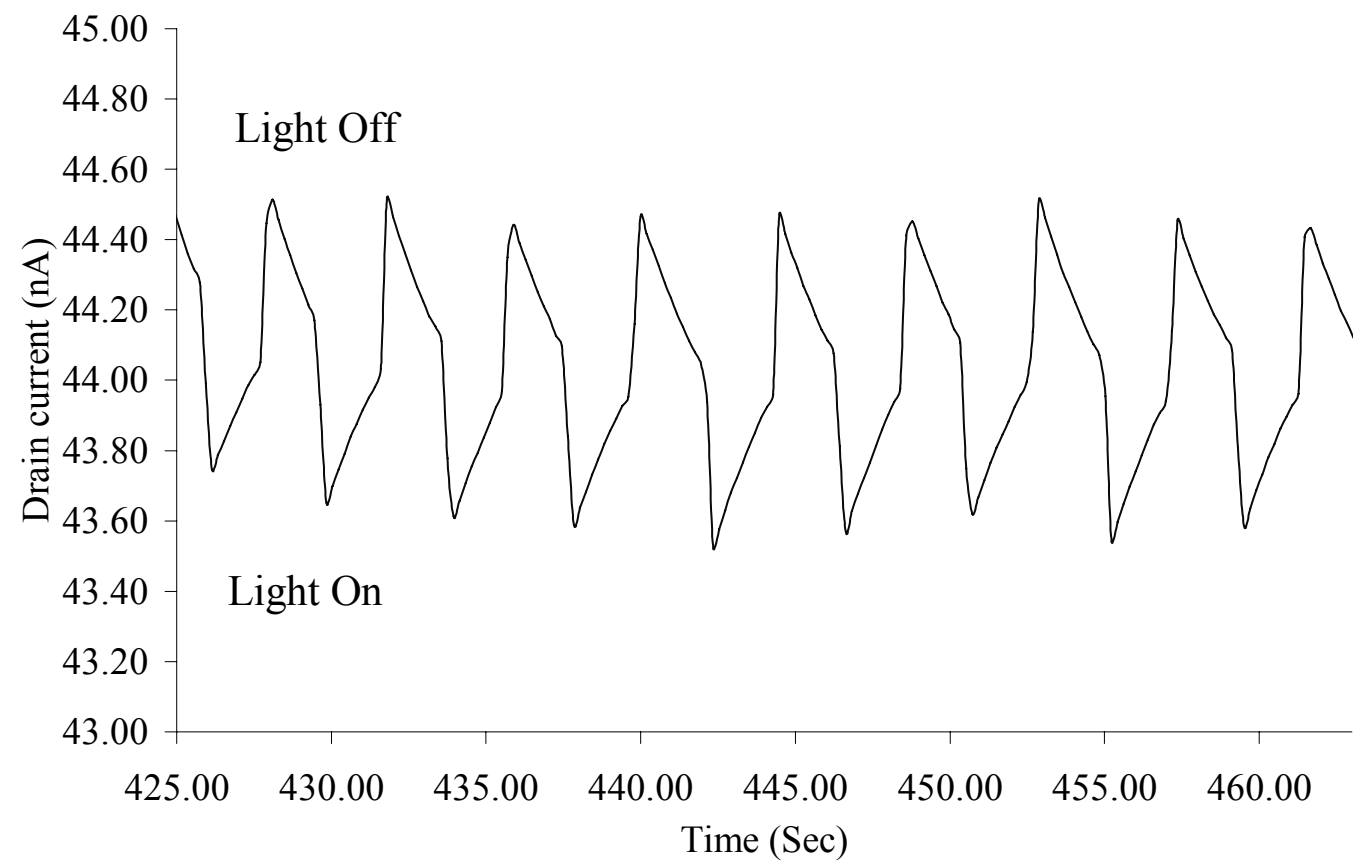

Figure 109. Current versus time measurements of a SET with bR interfaced with the gate and flashed at a flash rate of $0.4 \mathrm{~Hz}$. 


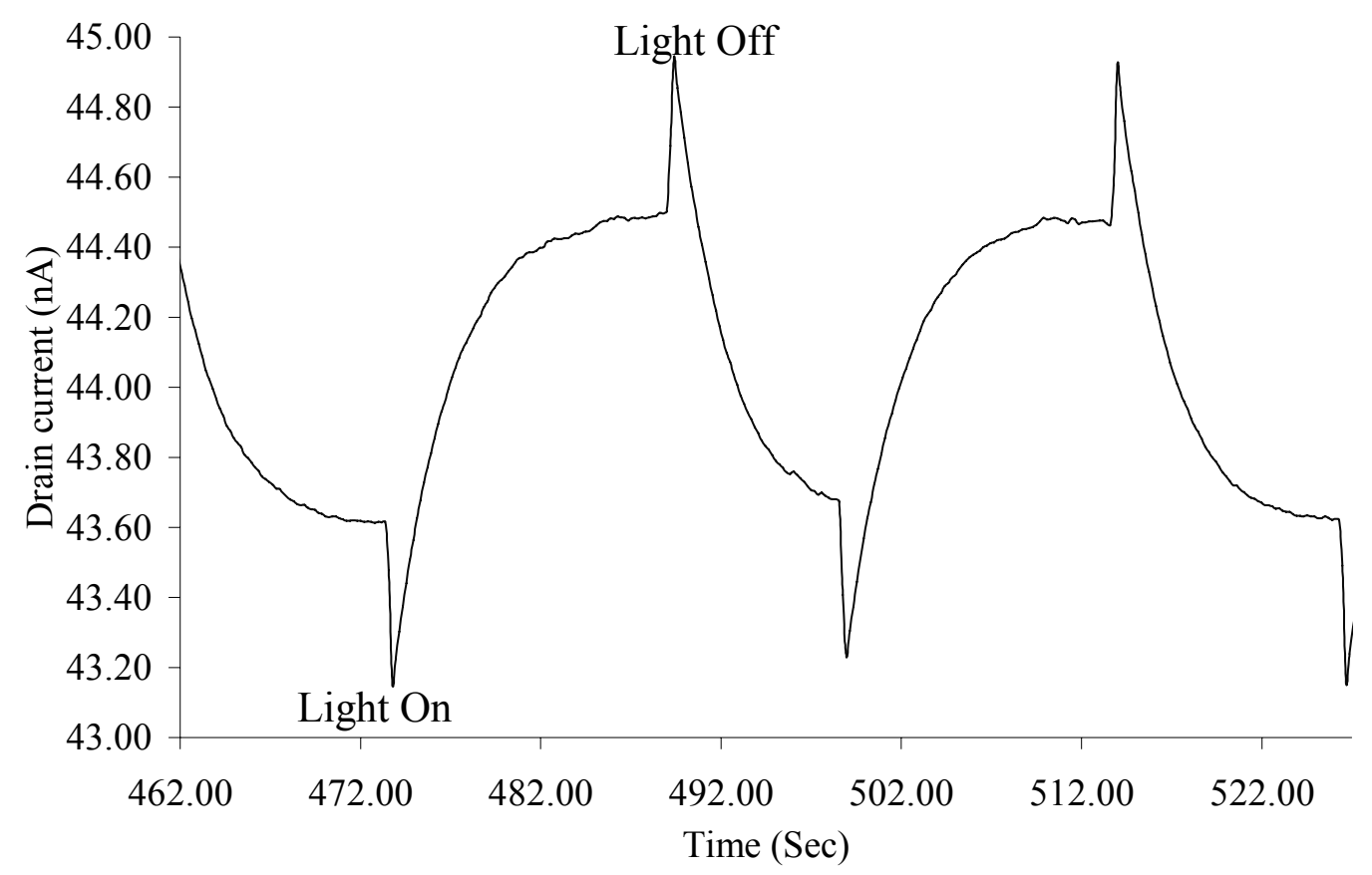

Figure 110. Current versus time measurements of a SET with bR interfaced with the gate and flashed at a flash rate of $60 \mathrm{mHz}$.

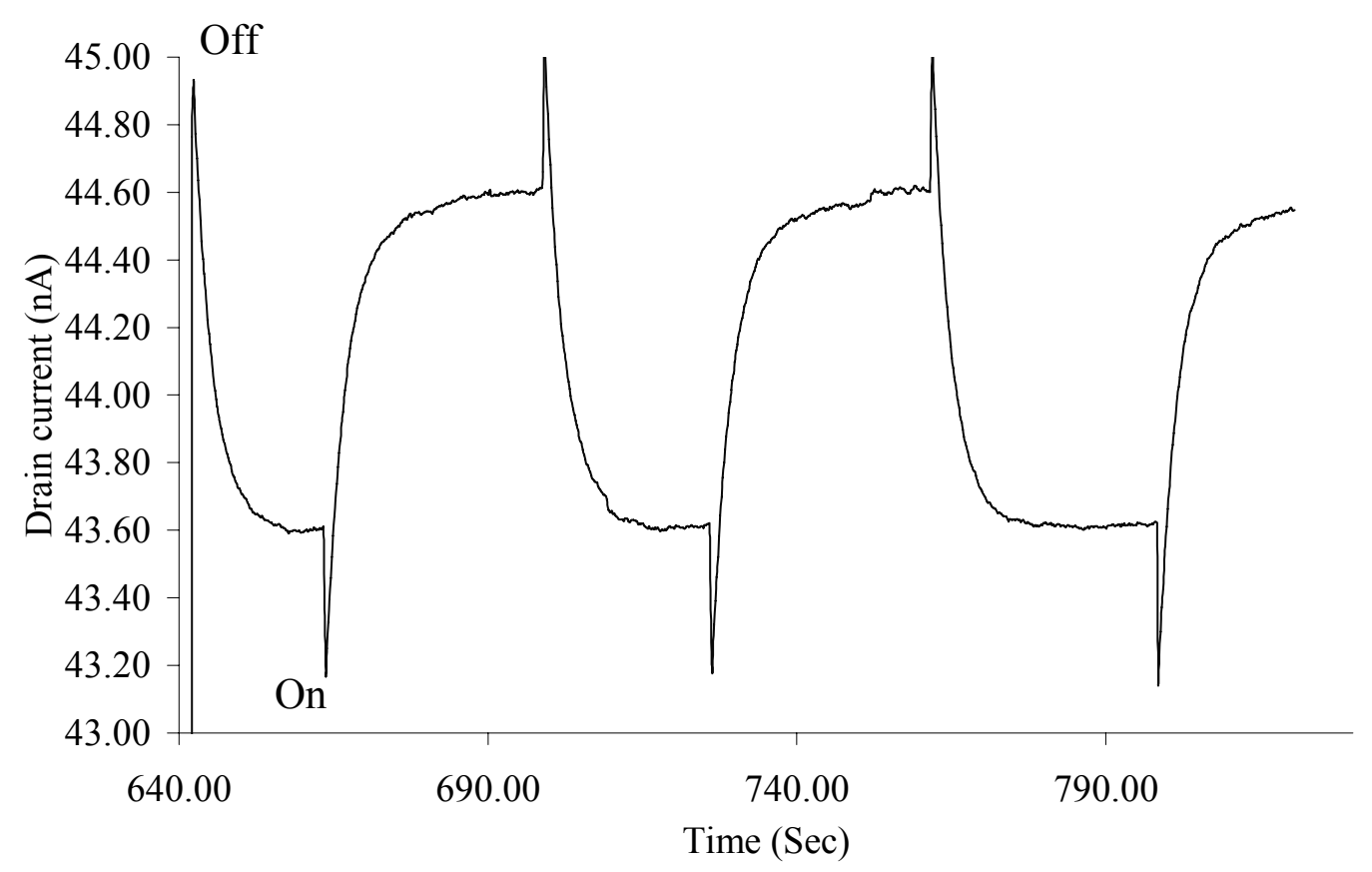

Figure 111. Current versus time measurements of a SET with bR interfaced with the gate and flashed at a flash rate of $12 \mathrm{mHz}$. 


\subsection{Activation of bR-SET with Sputter Gold Counter- Electrode}

In the previous section the bR did not have a top electrode and in this section the bR was sputter coated with a $5 \mathrm{~nm}$ layer of gold. The SETs were fabricated with a combination of sputtering, photolithography, and wet chemical etching, as previously described. The bR was electrophoretically deposited on the gate of the SET and then sputter coatd with 5 $\mathrm{nm}$ of gold. The 4200 SPA was configured as described in the previous section with one addition, the bR top gold electrode was ground during measurements. The thought was that grounding the $\mathrm{bR}$ will produce a larger voltage potential than the floating $\mathrm{bR}$ on the gate pad. The larger electric field will increase the possibility of modulating the tunneling of electrons between the source/drain gap.

I-V characteristic curves of the bR-SET without light are shown in Figure 112. The voltage and current generated by exciting bR on the gate of the SET caused a change in the current flowing between the source and drain of the device as shown in Figure $113-$ Figure 115 . To ensure that the change in current was due to the light activated $b R$ and not some other artifact, multiple tests of blocking the light source and altering the light intensity of the light source were conducted.

First, the light was blocked without moving the LEDs. Blocking the LEDs eliminated the current change across the source and drain as the LEDs were flashed. Then, the light intensity of the LEDs were varied since the voltage potential of $b R$ is dependent on the light intensity of the excitation source, the larger the light intensity the larger the voltage potential generated by bR. As shown in Figure 113 and Figure 115, changing the light intensity of the LEDs caused a change in the amount of current flowing between the source and drain. These graphs indicate increasing the light intensity output of the LEDs increases the change in current flowing between the source and drain.

Next to establish an understanding of the controllability of the SET the bias voltage on the source terminal was varied to attempt to control the current flowing between the source and drain. The bias voltages on the source terminal determined from the characteristic I-V curve can be varied from $-25 \mathrm{~V}$ to $25 \mathrm{~V}$. If the SET is working properly, changing the bias voltage on the source terminal should change the current flowing across the source-drain gap.

Within the Coulomb blockade, $-5 \mathrm{~V}$ to $5 \mathrm{~V}$, the current flowing from the source to the drain should be minimum. As shown in Figure 114, there was no measurable change in the current flowing from the source to the drain. Outside the coulomb blockade region the current from the source to the drain will flow and increase depending on the source bias voltage. As shown in Figure 115, the change in current is maximum at a measured range of $-20 \mathrm{~V}$ and minimum at $0 \mathrm{~V}$, which also shows that the current flow across the device can be controlled by altering the source bias voltage and/or by changing the light intensity activating the bR. 


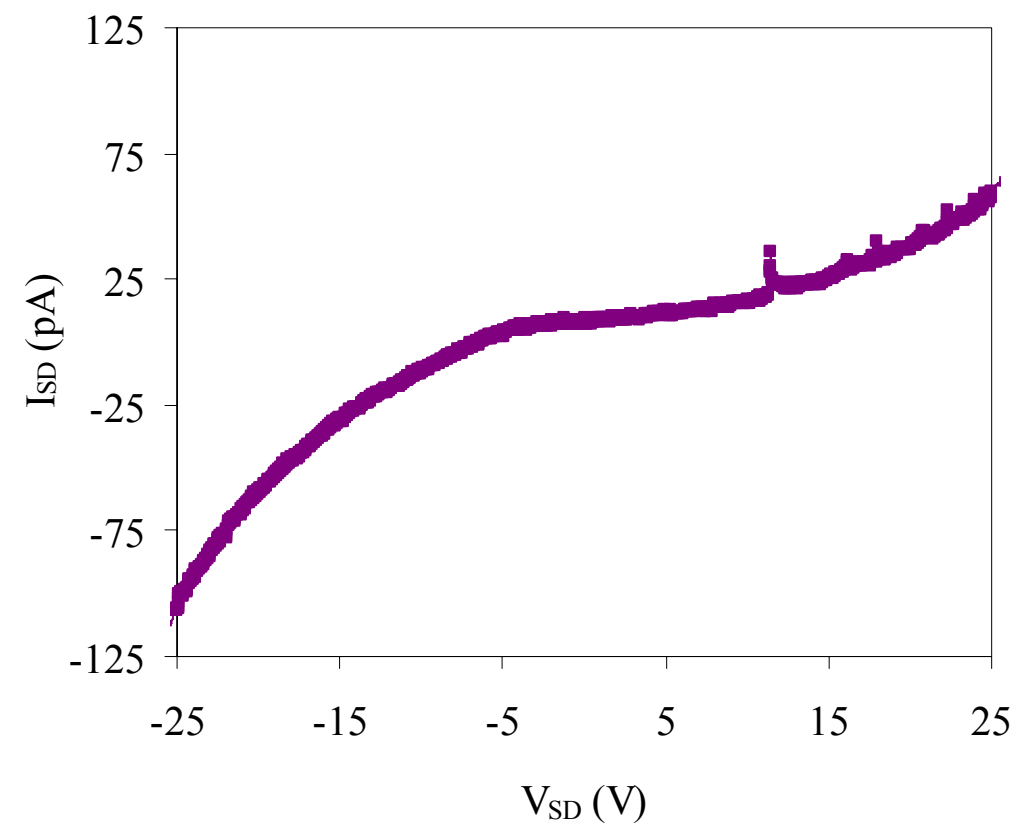

Figure 112. I-V curve of SET coupled with bR.

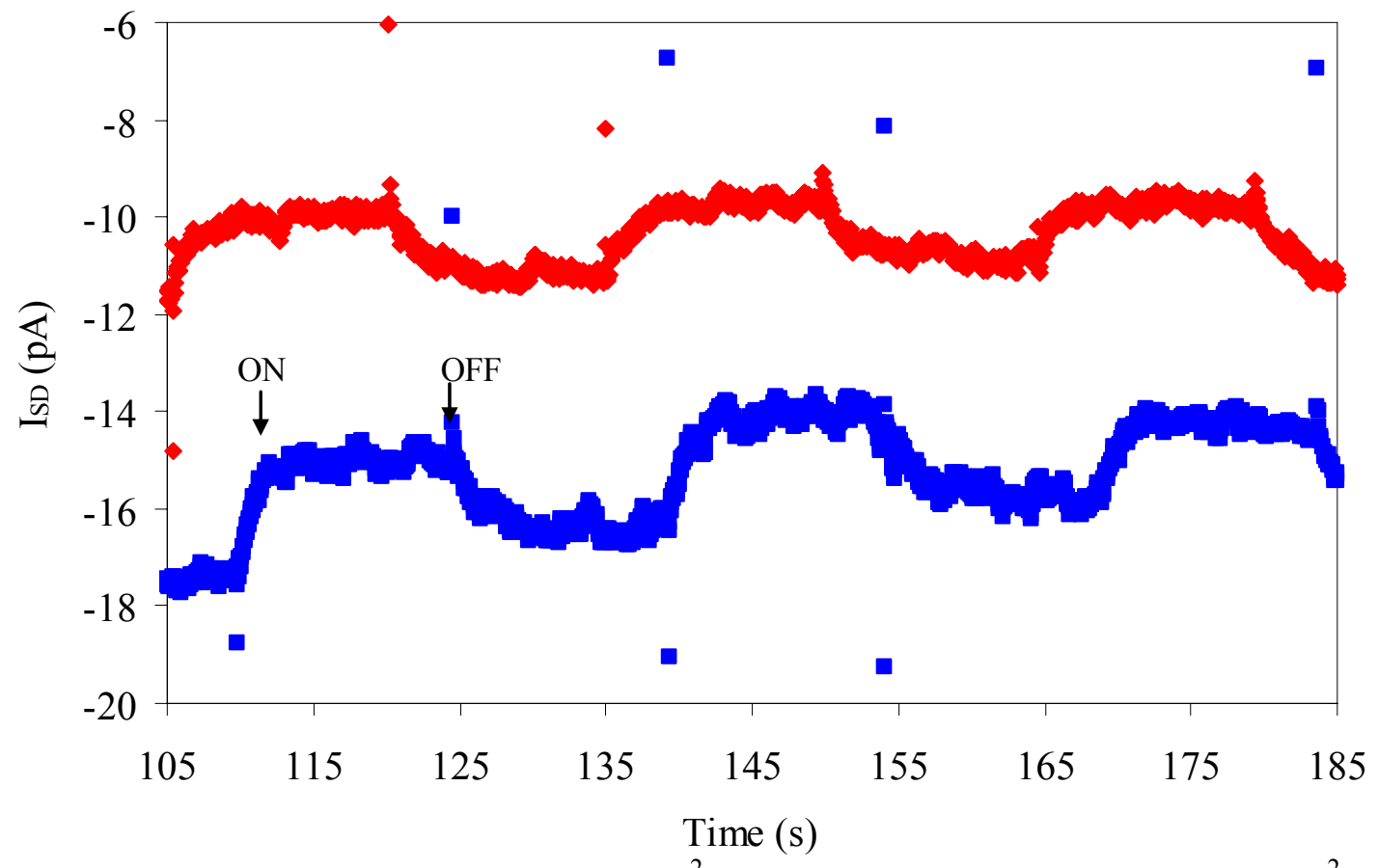

- Bias -20V Light Intensity $134 \mathrm{~W} / \mathrm{m}^{2}$ - Bias -20V Light Intensity $196 \mathrm{~W} / \mathrm{m}^{2}$

Figure 113. I-T curves of SET coupled with $b R$, bias with a source voltage of $-20 \mathrm{~V}$, and with a flashing light source at $134 \mathrm{~W} / \mathrm{m}^{2}$ (red) and $196 \mathrm{~W} / \mathrm{m}^{2}$ (blue). 


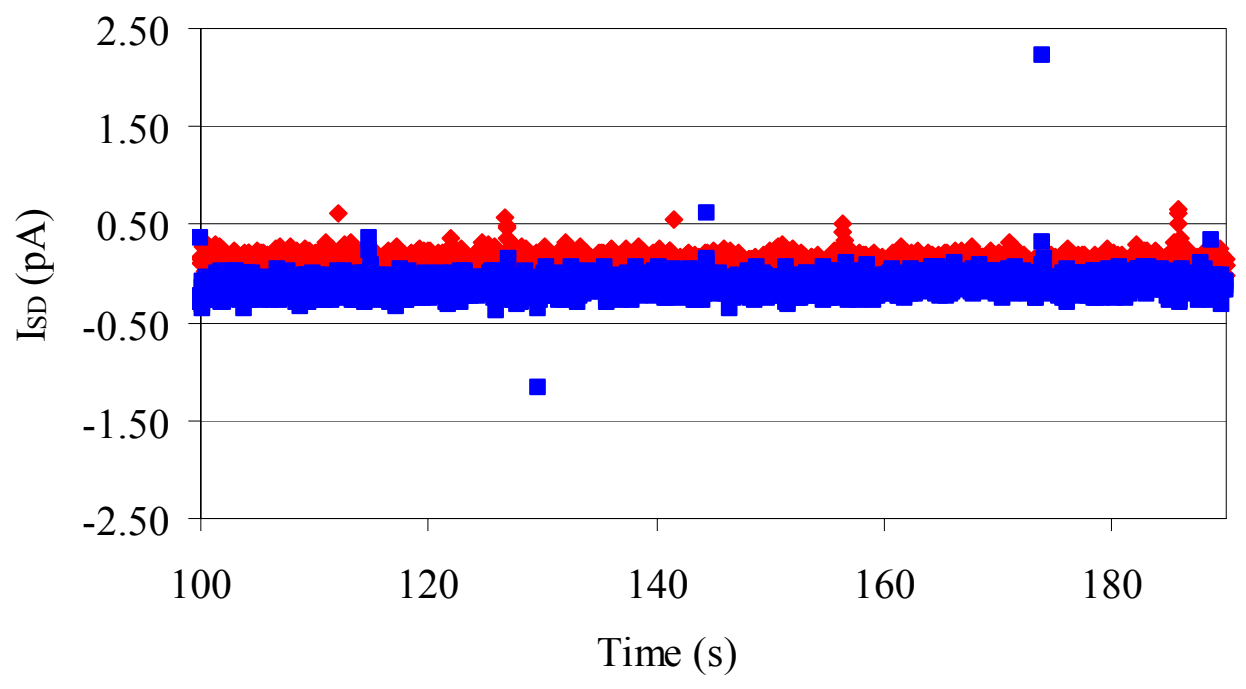

- Bias 0V Light Intensity $134 \mathrm{~W} / \mathrm{m}^{2}$ - Bias 0V Light Intensity $196 \mathrm{~W} / \mathrm{m}^{2}$

Figure 114. I-T curves of SET coupled with $\mathrm{bR}$, bias with a source voltage of $0 \mathrm{~V}$, and with a flashing light source at $134 \mathrm{~W} / \mathrm{m}^{2}$ (red) and $196 \mathrm{~W} / \mathrm{m}^{2}$ (blue).

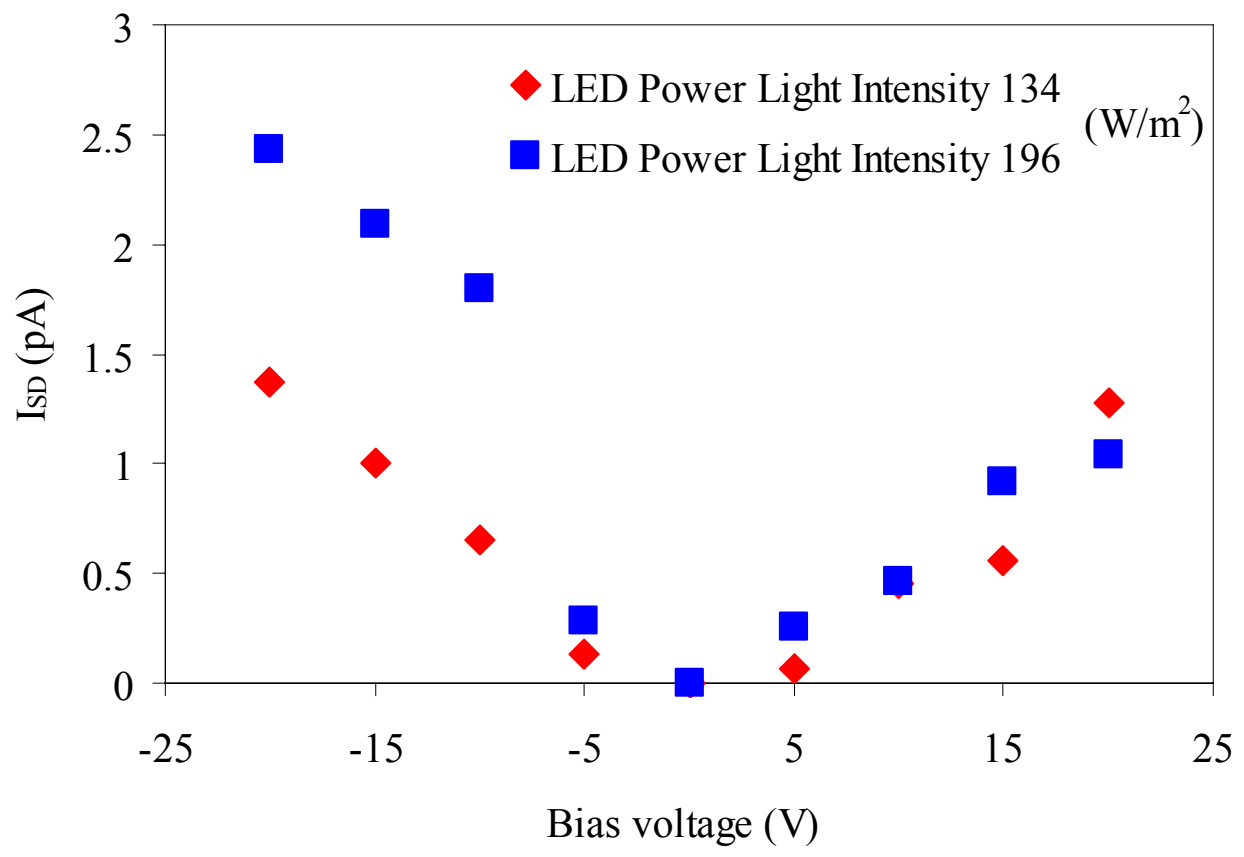

Figure 115. Change in current versus source bias voltage with a flashing light source at $134 \mathrm{~W} / \mathrm{m}^{2}$ (red) and $196 \mathrm{~W} / \mathrm{m}^{2}$ (blue). 


\section{Chapter 9 \\ Conclusions and Recommendations}

\subsection{Conclusions}

This research focused on the development of technology that could be used to create a platform for a chemical/biological sensor by integrating bacteriorhodopsin with a single electron transistor. To accomplish this research other research had to be conducted and processes developed. A process was developed to produce, isolate, and verify the purity of purple membrane. The verification step revealed that purple membrane produce was $\sim 90 \%$ purity which was accomplished at a fraction of the cost of commercially available PM.

The size of the PM is the limiting dimension for how small the device can be. Methods of decreasing the size of PM, with an average diameter of $500 \mathrm{~nm}$, were examined. Sonication had limited success in decreasing patch size but monomerization of PM was accomplished by solubilizing PM in OG. This was verified by a combination of centrifugation and gel column chromatography.

A process for integrating $b R$ with a SET was developed. The method used to deposit and orient bR on the gate of SETs was electrophoretic deposition, but Langmuir-Blodgett and ionic self-assembly are also other potential methods. Once deposited and oriented, a T $\Omega$ impedance op-amp circuit was developed to measure the photovoltaic response of $b R$. This circuit was also used to measure the electronic characteristics of $b R$.

The electronic characteristics of bR were measured using both a LCR meter and an opamp circuit. From these measurements it was determined that the resistance and capacitance both change when $\mathrm{bR}$ is light activated. It was found that the resistance decreases with increasing light intensity and decreased thickness of the sample. While the capacitance increases with increasing light intensity and decreasing thickness. The change in resistance and capacitance with light intensity was in both orient and unoriented samples. Also from this data, the relative permittivity and resistivity were estimated to be 2.2 and $2 \mathrm{G} \Omega-\mathrm{m}$. A representative empirical model of dried bR was suggested as a resistor and capacitor in parallel and fit to the photovoltaic data. Using the high impedance op-amp circuit, the photovoltaic responses were capture and analyzed at a variety of light intensities, sample thicknesses, and for both oriented and unoriented samples. From this, it was determined that the photovoltage increases linearly with respect to a linear increase in the light intensity. Analyzing the photovoltaic curves with the model developed revealed that the LARC decreases with increasing light intensity

and decreasing sample thickness, which indicates the fastest response time should occur 
with a monolayer of $b R$, suggesting that for a sensor application a monolayer of $b R$ should allow for quickest activation time to test for chemical/biological agents.

The SETs were fabricated by a combination of sputtering, photolithography, and focused ion beam machining. The SET gate pad served as the interface for the bR with the SET. The bR was then integrated with the SET by electrophoretic deposition, creating a bionanohybrid device. The bionanohybrid device was characterized using a 4200 SPA. Characterization demonstrated that the bR modulated the operational characteristics of the SET when bR was activated with light within its absorbance spectrum. There was no effort to optimize the SET or the fabrication process for use with $\mathrm{bR}$.

With this initial research into the integration of $b R$ with a single electron transistor, the measurement electronic characteristics of $b R$, and model development, it is proposed that this research should be further developed and eventually lead to the development of a multi-configurable toxin sensor.

\subsection{Recommendations}

A new process should be developed to improve the yield of functional SETs. This may be accomplished by finding a new method of creating QDs that reduces variability in size and density, or by optimizing SET architectures (e.g. vertical device where the source/drain overlap) while working towards a single dot device. The location of the gate electrode with respect to the source and drain (e.g. top gate, bottom gate, horizontal gate, separation distance) should be optimized to find the location of maximum controllability for a given gate voltage. This would improve the sensitivity of the device if a smaller gate voltage would modulate the tunneling of the electrons between the source and drain.

A second area to explore is to determine if solubilized $\mathrm{bR}$ is still photoactive. To accomplish this, a method of depositing and orienting the $\mathrm{bR}$ monomers needs to be developed. Monomerized bR maybe more sensitive and produce a larger photovoltage for a given area. This will be important for future sensor development.

A third area to explore is to measure the electronic properties of monolayers of PM and $\mathrm{bR}$. And also explore use of polarized light on the electrical properties of bR. From the presented research it was calculated that a monolayer would have the quickest response time. This should be verified and then integrated on the gate and characterized with different light intensity and possibly other environmental factor such as temperature and humidity, for both wet and dry films.

A fourth area to explore is to conduct research on energy harvesting with bR incorporated with other materials and structures. Experiments need to be conducted to explore the integration of both random and specifically attached nano-materials such as QDs, nano tubes, and metallic nano particles to $b R$ to see if they increase current generation. Other possible structures to explore for energy harvesting are porous materials such as doped silicon and titanium, where bR could be incorporated into the pores. 


\section{REFERENCES}

[1] www.itrs.net, "The international technology roadmap for semiconductors," in ITRS, 2008, Ed., 2008.

[2] N. Vsevolodov, Biomolecular Electronics an Introduction via Photosensitive Proteins Boston: Birkhauser, 1998.

[3] A. R. Clapp, I. L. Medintz, J. M. Mauro, B. R. Fisher, M. G. Bawendi, and H. Mattoussi, "Fluorescence resonance energy transfer between quantum dot donors and dye-labeled protein acceptors," J. Am. Chem. Soc., vol. 126, pp. 301-310, 2004.

[4] M. H. Griep, "Quantum dot/optical protein bio-nano hybrid system for biosensing applications " in Mechanical Engineering-Engineering Mechanics vol. Ph.D. Houghton: Michigan Technological University, 2008.

[5] D. Voet and J. Voet, Biochemistry 1ed. New York: John Wiley \& Sons, 1990.

[6] R. Birge, "Photophysics and molecular electronic applications of the rhodopsins," Ann. Rev. Phys. Chem., vol. 41, pp. 683-733, 1990.

[7] D. L. Nelson and M. M. Cox, Lehninger principles of biochemistry 4ed. New York: W.H. freeman and company, 2005.

[8] A. A. Kononenko, E. P. Lukashev, S. K. Chamorovsky, A. V. Maximychev, S. F. Timashev, L. N. Chekulaeva, A. B. Rubin, and V. Z. Paschenko, "Oriented purple-membrane films as a probe for studies of the mechanism of bacteriorhodopsin functioning. II. photoelectric processes," Biochemica et Biophysica Acta, vol. 892, pp. 56-67, 1987.

[9] N. A. Hampp, "Bacteriorhodopsin: Mutating a Biomaterial into an Optoelectronic Material," Appl. Microbiol. Biotechnol., vol. 53, pp. 633-639, 2000.

[10] N. Hampp, "Bacteriorhodopsin as a photochromic retinal protein for optical memories," Chem. Rev. , vol. 100, pp. 1755 - 1776, 2000.

[11] R. Jonas, Y. Koutalos, and T. G. Ebrey, "Purple Membrane: Surface charge density and the multiple effect of $\mathrm{pH}$ and cations," Photochemistry and Photobiology, vol. 52, pp. 1163-1177, 1990.

[12] J.-A. He, L. Samuelson, L. Li, J. Kumar, and S. K. Tripathy, "Bacteriorhodopsin thin film assemblies- Immobilization, properties, and applications," Advanced Materials, vol. 11, pp. 435-445, 1999.

[13] http://nobelprize.org/, "Nobelprize.org," 2008.

[14] P. Mitchell, "Coupling of phosphorylation to electron and hydrogen transfer by a chemi-osmotic type of mechanism," Nature, vol. 191, pp. 144-148, 1961.

[15] D. Oesterhelt and W. Stoeckenius, Isolation of the cell membrane of halobacterium halobium and its fractionation in to red and purple membrane, vol. 31. New York: Academic Press, 1974.

[16] J. Deisenhofer and H. Michel, "The photosysnthetic reaction centre from the purple bacterium Rhodopseudomonas viridis," Nobel Lecture, 1988.

[17] S.-B. Hwang, J. I. Korenbrot, and W. Stoeckenius, "Transinent Photovoltages in purple membrane multilayers charge displacement in bacteriorhodopsin and its photointermediates," Biochemica et Biophysica Acta, vol. 509, pp. 300-317, 1978. 
[18] M. Ikonen, "Bacteriorhodopsin in Langmuir-Blodgett films - preparation, sturcture and photoinduced optical and electrical properties," in Department of chemistry, physical chemistry vol. Ph.D. Helsinki: University fo Helsinki, 1993, pp. 27.

[19] C. Nicolini, V. Erokhin, S. Paddeu, and M. Sartore, "Towards a light-addressable transducer bacteriorhodopsin based," Nanotechnology, vol. 9, pp. 223-227, 1998.

[20] P. Bertoncello, D. Nicolini, C. Paternolli, V. Bavastrello, and C. Nicolini, "Bacteriorhodopsin-based langmuir-schaefer for solar energy capture," IEEE Transactions on NanoBioScience, vol. 2, pp. 124-132, 2003.

[21] L. Keszthelyi, "Orientation of membrane fragments by electric field," Biochemica et Biophysica Acta, vol. 598, pp. 429-436, 1980.

[22] W. Wang, G. Knopf, and A. Bassi, "Photoelectric Properties of a Detector Based on Dried Bacteriorhodopsin Film," Biosensors \& Bioelectronics, vol. 21, pp. 1309-1319, 2006.

[23] L. A. Samuelson, P. Miller, D. M. Galotti, K. A. Marx, j. kumar, s. k. tripathy, and d. L. kaplan, "The monomolecular organization of a photodynamic protein system through specific surface recongnition of streptavidin by biotinylated langmuir-blodgett films," Langmuir, vol. 8, pp. 604-608, 1992.

[24] K. Koyama, N. Yamaguchi, and T. Miyasaka, "Antibody-mediated bacteriorhodopsin orientation for molecular device architectures," Science, vol. 265, pp. 762-765, 1994.

[25] J. A. DeRose and R. M. Leblanc, "Scanning tunneling and atomic force microscopy studies of Langmuir-Blodgett films," Surface Science Reports, vol. 22, pp. 73-126, 1995.

[26] M. C. Petty, Langmuir-Blodgett Films an Introduction: Cambridge University Press, 1996.

[27] K. I. LTD, "Langmuir and langmuir-blodgett films what and how?," 2005.

[28] A. V. Maximychev, A. S. Kholmansky, E. V. Levin, N. G. Rambidi, S. K. Chamorovsky, A. A. Kononenko, V. V. Erokhin, and L. N. Checulaeva, "Oriented purple membrane mulitlayers of halobacteria fabricated by langmuir-blodgett and electrophoretic sedmentation techniques," Advanced Materials for Optics and Electronics, vol. 1, pp. 105-115, 1992.

[29] S.-B. Hwang, J. I. Kornebrot, and W. Stoeckenius, "Proton Transport by bacteriorhodopsin through an interface film," J. Membrane Biol., vol. 36, pp. 136$158,1977$.

[30] H. H. Weetall and A. B. Druzhko, "Optical and electrical characteristics of bacteriorhodopsin gelatin films and tin-oxide coated electrodes," Current Applied Physics, vol. 3, pp. 281-291, 2003.

[31] J. Boucher, E. Trudel, M. Methot, P. Desmeules, and C. Salesse, "Organization, structure and activity of proteins in monolayers," Colloids and Surfaces B: Biointerfaces, 2007.

[32] H. Choi, H. Lee, and C. Montemagno, "Toward hybrid proteo-polymeric vesicles generating a photoinduced proton gradient for biofuel cells," Nanotechnology, vol. 16, pp. 1589-1597, 2005. 
[33] M. Ikonen, A. Sharonov, N. Tkachenko, and H. Lemmetyinen, "The photovoltage signals of bacteriorhodopsin in langmuir-blodgett films with different molecular orientations," Advanced Materials for Optics and Electronics, vol. 2, pp. 115-122, 1993.

[34] H.-G. Choi, W.-C. Jung, J. Min, W. H. Lee, and J.-W. Choi, "Color image detection by biomolecular photoreceptor using bacteriorhodopsin-based complex LB films," Biosensors \& Bioelectronics, vol. 16, pp. 925-935, 2001.

[35] S.-B. Hwang and W. Stoeckenius, "Purple membrane vesicles: morphology and proton translocation," J. Membrane Biol., vol. 33, pp. 325-350, 1977.

[36] N. Yamaguchi, Y. Jinbo, M. Arai, and K. Koyama, "Visualization of the morphology of purple membrane surfaces by monoclonal antibody techniques," FEBS Letters, vol. 324, pp. 287-292, 1993.

[37] I. Langmuir and V. J. Schaefer, "Activities of unrease and pepsin monolayers," $J$. Am. Chem. Soc., vol. 60, pp. 1354-1360 1938.

[38] T. Miyasaka and K. Koyama, "Rectified photocurents from purple membrane langmuir-blodgett films at the electrode-electrolyte interface," Thin Solid Films, vol. 210-211, pp. 146-149, 1993.

[39] T. Miyasaka, Y. Maekawa, and K. Koyama, "A Novel Photoreactive amphiphile of nitrophenylazide for immobilization of bioactive proteins," Thin Solid Films, vol. 180, pp. 73-83, 1989.

[40] Y. Sugiyama, T. Inoue, M. Ikematsu, and M. Iseki, "Controlling the orientation of purple membrane fragments on an air/water interface by a new method of direct electric field application during purple membrane spreading," Jpn. J. Appl. Phys., vol. 36, pp. 5674-5679, 1997.

[41] K. A. Fisher, K. Yanagimoto, and W. Stoeckenius, "Oriented Adsorption of Purple Membrane to Cationic Surfaces," J. Cell. Biology, vol. 77, pp. 611-621, 1978.

[42] J.-A. He, L. Samuelson, L. Li, J. Kumar, and S. K. Tripathy, "Oriented bacteriorhodopsin/polycation multilayers by electrostatic layer-by-layer assembly," Langmuir, vol. 14, pp. 1674-1679, 1997.

[43] PDB, "Protein data bank," 2007.

[44] S. Freitag and R. Stenkamp, "Streptavidin Project Description," 2003.

[45] K. Koyama, N. Yamaguchi, and T. Miyasaka, "Molecular organization of bacteriorhodopsin films in optoelectronic devices," Advanced Materials, vol. 7, pp. 590-594, 1995.

[46] M. Green, AVIDIN, Advances in protein chemsity. New York, NY: Avidin, Ed. Academic Press, 1975.

[47] L. A. Bauer, N. S. Birenbaum, and G. J. Meyer, "Biological applications of high aspect ratio nanoparticles," J. Mater. Chem., vol. 14, pp. 517-526, 2004.

[48] D. Christensen, "Assembly and Characterization of Protein Multi-Layer Systems," in Max Planck Institute for Ploymer Research, vol. Masters: Johannes Gutenberg University-Mainz 2004, pp. 1-66.

[49] W. e. a. Knoll, "Supramolecular architectures for the functionalization of the solid surface," Advances in Biophysics, vol. 34, pp. 231-251, 1997. 
[50] W. e. a. Knoll, "Streptavidin arrays as supramolecular architechtures in surfaceplasmon optical sensor formats. ," Colloids and surfaces a-physicohemical and engineering aspects, vol. 161, pp. 115-137, 2000.

[51] D. Falconnet, G. Csucs, H. M. Grandin, and M. Textor, "Surface engineering approaches to micropattern surfaces for cell-based assays," Biomaterials, vol. 27, pp. 3044-3063, 2006.

[52] A. A. Dameron, J. R. Hampton, R. K. Smith, T. J. Mullen, S. D. Gillmor, and P. S. Weiss, "Microdisplacement Printing," Nano Letters, vol. 5, pp. 1834-1837, 2005.

[53] S. Crittenden, R. Reifenberger, J. Hillebrecht, R. Birge, D. Inerowicz, and F. Regnier, "Soft lithography based micron-scale electrophorectic patterning of purple membrane," J. Micromech. Microeng. , vol. 15, pp. 1494-1497, 2005.

[54] C. M. Anton, "Photolithography Based Patterning of Bacteriorhodopsin Films," in Mechanical Engineering-Engineering Mechanics vol. Ph.D. Houghton: Michigan Technological University, 2008, pp. 186.

[55] D. Haronian and A. Lewis, "Microfabricating bacteriorhodopsin films for imaging and computing," Appl. Phys. Lett., vol. 61, pp. 2237-2239, 1992.

[56] S. Libertino, M. Fichera, G. D'Arrigo, A. L. Mantia, and D. Ricceri, "Characterization and pattering of bacteriorhodopsin films on Si-based materials," Synthetic Metals, vol. 138, pp. 71-74, 2003.

[57] T. Miyasaka and K. Koyama, "Image sensing and processing by a bacteriorhodopsin-based artificial photoreceptor," Applied Optics, vol. 32, pp. 6371-6379, 1993.

[58] P. S. K. Karre, P. L. Bergstrom, G. Mallick, and S. P. Karna, "Room temperature operational single electron transistor fabricated by focused ion beam deposition " Journal of Applied Physics, vol. 102, pp. 1-4, 2007.

[59] K. Yasuo, I. Kazumasa, Y. Ryo-taro, I. Ken-ichi, I. Kingo, and N. Michio, "Room-temperature observation of a Coulomb blockade phenomenon in aluminum nanodots fabricated by an electrochemical process," Applied Physics Letters, vol. 90, pp. 093119, 2007.

[60] V. Ray, R. Subramanian, P. Bhadrachalam, L.-C. Ma, C.-U. Kim, and S. J. Koh, "CMOS-compatible fabrication of room-temperature single-electron devices," Nat Nano, vol. 3, pp. 603-608, 2008.

[61] D. Berman, "The aluminum single-electron transistor for ultrasensitive electrometry of semiconductor quantum-confined systems," in Electrical Engineering and computer science, vol. ph.D. Masssachusetts: MIT, 1998, pp. 1137.

[62] M. A. Kastner, "The single electron transistor and artifical atoms," Ann. Phys., vol. 9, pp. 885-894, 2000.

[63] K. K. Likharev, "SET: coloumb blockade devices," Nano et Micro Technologies, vol. 3, 2003.

[64] R. A. Smith and H. Ahmed, "A silicon Coulomb blockade device with voltage gain," Applied Physics Letters, vol. 71, pp. 3838-3840, 1997.

[65] G. Zimmerli, R. L. Kautz, and M. M. John, "Voltage gain in the single-electron transistor," Applied Physics Letters, vol. 61, pp. 2616-2618, 1992. 
[66] S. Nomura, N. Kajimura, K. Miyata, R. Ortenberg, M. Mevarech, H. Kamikubo, M. Karaoka, and Y. Harada, "Ordered structure formation of BacteriorhodopsinhDHFR in a plasma membrane," Langmuir, vol. 15, pp. 214-220, 1999.

[67] S. Fleischer and L. Packer, "Biomembranes," Methods in Enzymology, 1974.

[68] S. Tristram-Nagle, C.-P. Yang, and J. F. Nagle, "Thermodynamic studies of purple membrane," Biochemica et Biophysica Acta, vol. 854, pp. 58-66, 1986.

[69] N. Vasevolodov, Biomulecular electronics an introduction via photosensitive proteins 1998.

[70] J. Bridgen and I. D. Walker, "Photoreceptor protein from the purple membrane of halobacterium halobium. Molecular weight and retinal binding site," Biochemistry, vol. 15, pp. 792-798, 1976.

[71] N. A. Dencher and M. P. Heyn, "Preparation and properties of monomeric bacteriorhodopsin," Methods of Enzmology, vol. 88, pp. 5-10, 1982.

[72] M. N. Jones and D. Chapman, Micelles, monolayers, and biomembranes. New York: Wiley-Liss, Inc, 1995.

[73] N. M. Green, "Spectrophotometric determination of avidin and biotin," Methods of Enzmology, 1978.

[74] D.-1. Chen, Y.-j. Lu, S.-f. Sui, B. Xu, and K.-s. Hu, "Oriented assembly of purple membrane on solid support mediated by molecular recognition," J. Phys. Chem, vol. 107, pp. 3598-3605, 2003.

[75] J. Wang, "Photocurrent from oriented membrane films containing acid-blue and acid-purple bacteriorhodopsin and its mutants," Photochemistry and Photobiology, vol. 71, pp. 476-480, 2000.

[76] A. I. Stognij, N. N. Novitskii, S. D. Tushina, and S. V. Kalinnikov, "Preparation of ultrathin gold films by oxygen-ion sputtering and their optical properties," Technical Physics, vol. 48, pp. 745-748, 2003.

[77] M. E. D. Martin and R. L. Cerro, "Surface potentials and ionization equilibrium in Y-type deposition of multiple langmuir-blodgett films I. effect of $\mathrm{pH}$ and counterions," Journal of Colloid and Interface Science, vol. 285, pp. 686-695, 2005.

[78] Y. Jin, T. Honig, I. Ron, N. Friedman, M. Sheves, and D. Cahen, "Bacteriorhodopsin as an electronic conduction medium for biomolecular electronics," Chem Soc Rev, vol. 37, pp. 2442-2432, 2008.

[79] Y. Jin, N. Friedman, M. Sheves, T. He, and D. Cahen, "Bacteriorhodopsin (bR) as an electronic conduction medium: Current transport through bR-containing monolayers," PNAS, vol. 103, pp. 8601-8606, 2006.

[80] R. Garcia, J. Tamayo, J. Soler, and C. Bustamante, "Physical Parameters That Control the Imaging of Purple Membranes with the Scanning Tunneling Microscope," Langmuir, vol. 11, pp. 2109-2114, 1995.

[81] J. Xu, P. Bhattacharya, D. L. Marcy, J. A. Stuart, and R. R. Birge, "Photoconduction in bacteriorhodopsin/GaAs heterostructures," Electronics Letters, vol. 37, pp. 648-649, 2001.

[82] R. C. Advincula and M.-k. Park, "Ultrathin Films of Oriented Bacteriorhodopsin: Nanostructured Films for Investigating the Primary Photoevent in Vision Processes," Mat. Res. Soc. Symp. Proc., vol. 679E, pp. B3.2.1-B3.2.6, 2001. 
[83] M. Ikonen, A. Y. Sharonov, N. V. Tkachenko, and H. Lemmetyinen, "The kinetics of charges in dry bacteriorhodopsin langmuir-blodgett films-an analysis and comparison of electrical and optical signals," Advanced Materials for Optics and Electronics, vol. 2, pp. 211-220, 1993.

[84] S. Crittenden, S. Howell, R. Reifenberger, J. Hillebrecht, and R. R. Birge, "Humidity-dependent open-circuit photovoltage from a bacteriorhodopsin/indium tin oxide bioelectronic heterostructure," Nanotechnology, vol. 14, pp. 562-565, 2003.

[85] P. Lumileds, "Benefits of philips lumileds solid state lighting solutions vs. conventional lighting," Jan 20092009.

[86] P. S. K. Karre, "Frabrication and charaterization of room temperature operating single electron transistors using focused ion beam technologies," in Electrical Engineering vol. Ph.D. Houghton: Michigan Technological University, 2008, pp. 188.

[87] M. Acharya, "Development Of Room Temperature Operating Single Electron Transistors by FIB etch and deposition Process," vol. Ph.D. Houghton: Michigan Technological Univeristy, 2009.

[88] L. A. Giannuzzi and F. Stevie, Introduction to focused ion beams: instrumentation, theory, techniques, and practice, vol. 1. New York: Springer Science, 2005.

[89] M. Ishida, J. Fujita, T. Ichihashi, Y. Ochiai, T. Kaito, and S. Matsui, "Focused ion beam-induced fabrication of tungsten structures," Journal of Vacuum Science \& Technology B: Microelectronics and Nanometer Structures, vol. 21, pp. 27282731, 2003.

[90] M. Shinji, K. Takashi, F. Jun-ichi, K. Masanori, K. Kazuhiro, and H. Yuichi, "Three-dimensional nanostructure fabrication by focused-ion-beam chemical vapor deposition," 2000.

[91] J. Orloff, M. Utlaut, and L. Swanson, High resolution focused ion beams: FIB and its applications. New York: Kluwer Academic/Plenum Pbulishers, 2003.

[92] A. K. Kulkarni and L. C. Chang, "Electrical and structural characteristics of chromium thin films deposited on glass and alumina substrates," Thin Solid Films, vol. 301, pp. 17-22, 1997.

[93] M. H. F. Overwijk and F. C. van den Heuvel, "Focused-ion-beam-induced tungsten deposition: theory and experiment," Nuclear Instruments and Methods in Physics Research vol. 80, pp. 1324-1327, 1993.

[94] A. S. Cordan, Y. Leroy, A. Goltzene, A. Pepin, C. Vieu, M. Mejias, and H. Launois, "Temperature behavior of multiple tunnel junction devices based on disordered dot arrays," Journal of Applied Physics, vol. 87, pp. 345-352, 2000.

[95] A. S. Cordan, A. Goltzene, Y. Herve, M. Mejias, C. Vieu, and H. Launois, "Electron transport in metallic dot arrays: Effect of a broad dispersion in the tunnel junction dimensions," Journal of Applied Physics, vol. 84, pp. 3756-3763, 1998.

[96] J. Greer, A. Korkin, and J. Labanowski, Nano and giga challenges in microelectronics, 1 ed: ELSEVIER, 2003. 


\title{
PERMISSION STATEMENT FROM PUBLISHER
}

\author{
AMERICAN INSTITUTE OF PHYSICS LICENSE \\ TERMS AND CONDITIONS
}

Feb 05, 2009

This is a License Agreement between Karl A Walczak ("You") and American Institute of Physics ("American Institute of Physics") provided by Copyright Clearance Center ("CCC"). The license consists of your order details, the terms and conditions provided by American Institute of Physics, and the payment terms and conditions.

All payments must be made in full to CCC. For payment instructions, please see information listed at the bottom of this form.

License Number 2122611503444

License date Feb 05, 2009

Licensed content publisher American Institute of Physics

Licensed content title A silicon Coulomb blockade device with voltage gain

Licensed content author R. A. Smith

Type of Use Republish Portions

Requestor Type Third Party

Include other media

Number of portions 1

Title of your work Immobilizing bacteriorhodopsin on a single electron transistor

Publisher of your work UMI

Publication date of your work 02/20/2009

Billing Type

Invoice

Company

Karl A Walczak

Billing Address

1400 Townsend Drive, Department of MEEM

Houghton, MI 49931

United States

Customer reference info

Total

$\$ 0.00$

Terms and Conditions

American Institute of Physics -- Terms and Conditions: Permissions Uses

American Institute of Physics ("AIP") hereby grants to you the non-exclusive right and license to use and/or distribute the Material according to the use specified in your order, 
on a one-time basis, for the specified term, with a maximum distribution equal to the number that you have ordered. Any links or other content accompanying the Material are not the subject of this license.

1. You agree to include the following copyright and permission notice with the reproduction of the Material: "Reprinted with permission from Copyright American Institute of Physics." For an article, the copyright and permission notice must be printed on the first page of the article or book chapter. For photographs, covers, or tables, the copyright and permission notice may appear with the Material, in a footnote, or in the reference list.

2. If you have licensed reuse of a figure, photograph, cover, or table, it is your responsibility to ensure that the material is original to AIP and does not contain the copyright of another entity, and that the copyright notice of the figure, photograph, cover, or table does not indicate that it was reprinted by AIP, with permission, from another source. Under no circumstances does AIP, purport or intend to grant permission to reuse material to which it does not hold copyright.

3. You may not alter or modify the Material in any manner. You may translate the Material into another language only if you have licensed translation rights. You may not use the Material for promotional purposes. AIP reserves all rights not specifically granted herein.

4. The foregoing license shall not take effect unless and until AIP or its agent, Copyright Clearance Center, receives the Payment in accordance with Copyright Clearance Center Billing and Payment Terms and Conditions, which are incorporated herein by reference.

5. AIP or the Copyright Clearance Center may, within two business days of granting this license, revoke the license for any reason whatsoever, with a full refund payable to you. Should you violate the terms of this license at any time, AIP, American Institute of Physics, or Copyright Clearance Center may revoke the license with no refund to you. Notice of such revocation will be made using the contact information provided by you. Failure to receive such notice will not nullify the revocation.

6. AIP makes no representations or warranties with respect to the Material. You agree to indemnify and hold harmless AIP, American Institute of Physics, and their officers, directors, employees or agents from and against any and all claims arising out of your use of the Material other than as specifically authorized herein.

7. The permission granted herein is personal to you and is not transferable or assignable without the prior written permission of AIP. This license may not be amended except in a writing signed by the party to be charged.

8. If purchase orders, acknowledgments or check endorsements are issued on any forms containing terms and conditions which are inconsistent with these provisions, such inconsistent terms and conditions shall be of no force and effect. This document, including the CCC Billing and Payment Terms and Conditions, shall be the entire agreement between the parties relating to the subject matter hereof. 
This Agreement shall be governed by and construed in accordance with the laws of the State of New York. Both parties hereby submit to the jurisdiction of the courts of New York County for purposes of resolving any disputes that may arise hereunder.

Gratis licenses (referencing \$0 in the Total field) are free. Please retain this printable license for your reference. No payment is required.

If you would like to pay for this license now, please remit this license along with your payment made payable to "COPYRIGHT CLEARANCE CENTER" otherwise you will be invoiced within 30 days of the license date. Payment should be in the form of a check or money order referencing your account number and this license number 2122611503444.

If you would prefer to pay for this license by credit card, please go to http://www.copyright.com/creditcard to download our credit card payment authorization form.

Make Payment To:

Copyright Clearance Center

Dept 001

P.O. Box 843006

Boston, MA 02284-3006

If you find copyrighted material related to this license will not be used and wish to cancel, please contact us referencing this license number 2122611503444 and noting the reason for cancellation.

Questions? customercare@copyright.com or 877-622-5543 or +1-978-646-2777. 